\title{
The Processing and Storage of Information in Neuronal Memory Systems Across Time Scales
}

\author{
Dissertation \\ for the award of the degree \\ "Doctor rerum naturalium" \\ of the Georg-August-Universität Göttingen
}

within the doctoral program

International Max Planck Research School on Physics

of Biological and Complex Systems

of the Göttingen Graduate School for Neurosciences,

Biophysics, and Molecular Biosciences

submitted by

Timo Nachstedt

from Clausthal-Zellerfeld

Göttingen, 2017 


\section{Thesis Committee}

Dr. Christian Tetzlaff

III. Physikalisches Institut, Georg-August-Universität Göttingen

Prof Dr. Dr. Detlev Schild

Institut für Neurophysiologie und Zelluläre Biophysik, Universitätsmedizin Göttingen

Prof. Dr. Ulrich Parlitz

Max-Planck-Institut für Dynamik und Selbstorganisation

\section{Members of the Examination Board}

Referee: Dr. Christian Tetzlaff

III. Physikalisches Institut, Georg-August-Universität Göttingen

$2^{\text {nd }}$ Referee: Prof. Dr. Dr. Detlev Schild

Institut für Neurophysiologie und Zelluläre Biophysik, Universitätsmedizin Göttingen

\section{Further Members of the Examination Board}

Prof. Dr. Jörg Enderlein

III. Physikalisches Institut, Georg-August-Universität Göttingen

Prof. Dr. Stefan Klumpp

Institut für Nichtlineare Dynamik, Georg-August-Universität Göttingen

Prof. Dr. Ulrich Parlitz

Max-Planck-Institut für Dynamik und Selbstorganisation

Prof. Dr. Fred Wolf

Max-Planck-Institut für Dynamik und Selbstorganisation

Date of the oral examination: $27^{\text {th }}$ of November, 2017 


\section{The Processing and Storage of Information in Neuronal Memory}

Systems Across Time Scales

Dissertation for the award of the degree "Doctor rerum naturalium" of the Georg-August-Universität Göttingen

within the doctoral program International Max Planck Research School on Physics of Biological and Complex Systems of the Göttingen Graduate School for Neurosciences, Biophysics, and Molecular Biosciences submitted by Timo Nachstedt from Clausthal-Zellerfeld

Göttingen, 2017 
Revised version, May 2018.

This thesis contains McGraw-Hill Education Material. McGraw-Hill Education makes no representations or warranties as to the accuracy of any information contained in the McGraw-Hill Education Material, including any warranties of merchantability or fitness for a particular purpose. In no event shall McGraw-Hill Education have any liability to any party for special, incidental, tort, or consequential damages arising out of or in connection with the McGraw-Hill Education Material, even if McGraw-Hill Education has been advised of the possibility of such damages. 


\section{Abstract}

Humans and animals are able to store and recall information about past experiences across a variety of time scales. This process is accomplished by memory, which is implemented in the neuronal systems of the brain. These neuronal systems consist of a large number of electrically excitable cells, called neurons, which interact via contact points called synapses. The transmission efficacies of these synapses can be adapted by processes summarized as synaptic plasticity.

Working memory (WM) describes the ability to store and to process information on time scales from seconds up to a minute and is important in many cognitive processes. The neuronal mechanisms underlying WM are still not understood. Some experimental and theoretical studies suggest that the neuronal system which implements WM stores information in the form of persistent activity of specific groups of neurons. These stable activity configurations are called attractor states. Other studies suggest that the information is stored in the form of complex temporal sequences of various activity patterns, so called transient trajectories. In this thesis, we show that the neuronal system implementing WM actually depends on both transient neuronal activity as well as distinct attractor states. Furthermore, we demonstrate that these attractor states may emerge in a self-organized way in the neuronal system implementing long-term memory (LTM) that stores information on time scales from hours to years. Finally, we develop a mechanism that may allow transient neuronal activity in the WM system to control long-lasting time-dependent output signals.

First, we show that, different from human subjects, a model of a neuronal system which solely operates on transient activity dynamics is not able to solve a typical WM task with unpredictable temporal structure. Remarkably, the performance of this system is restored by introducing distinct attractor states into the system dynamics. Still, the transient trajectories in between these attractor states are required to enable non-linear time-dependent processing. Thus, the neuronal system which implements WM requires both transient dynamics and distinct attractor states. Second, we demonstrate that these attractor states can be created by groups of strongly interconnected neurons, so called cell assemblies (CAs), formed in the neuronal system implementing LTM. We show that CAs may be reliably formed and allocated to different stimuli by an interplay of two synaptic plasticity processes. Hence, the attractor states required by the WM system may emerge in a self-organized way in the LTM system. Third, we present a mechanism which enables a short transient signal to adapt the autonomously produced periodic output signal of neuronal systems called central pattern generators (CPGs). This mechanism allows to fast and precisely adapt the frequency of general oscillatory systems in a self-organized way based on the frequency of a short periodic stimulation. Thus, it enables short-lasting transient trajectories in the WM system to evoke long-lasting time-dependent neuronal signals.

In summary, we show that to allow for WM that is robust with respect to unpredictable temporal structure and can perform complex non-linear processing, the underlying neuronal system has to rely on a combination of transient trajectories and distinct attractor states. These attractor states may emerge in a self-organized way in the LTM system and in CPGs. 



\section{Contents}

1 Introduction 1

2 Experimental and Theoretical Background 5

2.1 Neuronal Networks of the Brain . . . . . . . . . . . . . . 5

2.2 The Hebbian Postulates . . . . . . . . . . . . . . . . . . 9

2.3 Long-Term Memory . . . . . . . . . . . . . . . . . . . . . 10

2.3.1 Neural Correlates of Long-Term Memory . . . . . . . . . . . . . 11

2.3.2 Neurocomputational Models of Long-Term Memory . . . . . . 11

2.4 Working Memory . . . . . . . . . . . . . . . . . . . 12

2.4.1 The N-Back Task as a Measure of Working Memory . . . . . . . 14

2.4.2 Neural Correlates of Working Memory . . . . . . . . . . . . . . 15

2.4.3 Neurocomputational Models of Working Memory . . . . . . . . 15

2.4 .4 Reservoir Networks . . . . . . . . . . . . . . . . . . . 16

2.5 Memory of Rhythmic Neuronal Signals . . . . . . . . . . . . . . . . 17

2.5.1 Central Pattern Generators . . . . . . . . . . . . . . . . . 18

2.5.2 Adaptive Frequency Oscillators . . . . . . . . . . . . . 18

3 Neuronal Dynamics of Working Memory 21

3.1 Methods ........................... 22

3.1.1 Recurrent Networks at the Edge of Chaos . . . . . . . . . . . . 22

3.1.2 Reservoir Network Training Algorithms . . . . . . . . . . . . 24

3.1.3 An N-Back Task for Reservoir Networks . . . . . . . . . . . . . 27

3.1.4 Principal Component Analysis . . . . . . . . . . . . . . . . . . 29

3.2 Performance of Reservoir Networks in the N-Back Task . . . . . . . . . 29

3.2.1 Predictable Stimulus Timing . . . . . . . . . . . . . . . 30

3.2.2 Unpredictable Stimulus Timing . . . . . . . . . . . . . . . . . 31

3.2.3 Restoring Performance with Additional Readout Units . . . . . 35

3.2.4 Dynamics of Principal Components . . . . . . . . . . . . . . . 36

3.2.5 Transient Dynamics and the Generation of Time-Dependent

Signals . . . . . . . . . . . . . . . 39

3.3 Discussion . . . . . . . . . . . . . . . 40

4 Self-Organized Formation and Allocation of Cell Assemblies 43

4.1 Methods . . . . . . . . . . . . . . . . . 44

4.1 .1 Network Model . . . . . . . . . . . . . . . . . . . . . . . . 44

4.1.2 Topological and Functional Measures . . . . . . . . . . . . . . . . 48

4.1.3 Nullclines, Equilibria and Asymptotic Stability . . . . . . . . . . 50

4.2 Cell Assembly Dynamics . . . . . . . . . . . . . . . . . . . . . . . 51

4.2.1 General Cell Assembly Formation . . . . . . . . . . . . . . 51

4.2 .2 Topological Properties . . . . . . . . . . . . . . . . . 53

4.2.3 Pattern Completion and Separation . . . . . . . . . . . . 57

4.2.4 Formation of Multiple Cell Assemblies . . . . . . . . . . . . . . 60

4.3 Population Model . . . . . . . . . . . . . . . . . . . . . . . . 63 
4.3.1 Model Definition . . . . . . . . . . . . . . . . . . . . . . . 63

4.3.2 Population Nullclines and Equilibria . . . . . . . . . . . . . . 65

4.3.3 Relevance of Feedforward Plasticity . . . . . . . . . . . . . . 69

4.3.4 Summary of the Formation and Assignment of Cell Assemblies 72

4.4 Discussion . . . . . . . . . . . . . . . 73

5 Fast Adaptation of Central Pattern Generators $\quad 77$

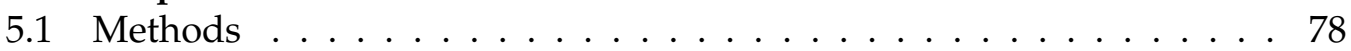

5.1 .1 Oscillators . . . . . . . . . . . . . . . . . 78

5.1 .2 Adaptive Frequency Oscillators . . . . . . . . . . . . . . . . 79

5.1 .3 Adaptation Quality Indicators . . . . . . . . . . . . . . . . . . . 82

5.1 .4 Numerical Integration . . . . . . . . . . . . . . . . . 83

5.2 Deficits of the AFO Mechanism . . . . . . . . . . . . . . . 84

5.2.1 Trade-off between Speed and Precision . . . . . . . . . . . . . 84

5.2.2 Adaptations Within a Wide Frequency Range . . . . . . . . . . . 85

5.3 Adaptation Through Fast Dynamical Coupling . . . . . . . . . . . . . 87

5.3.1 Derivation of the Mechanism . . . . . . . . . . . . . . 87

5.3.2 Examples of Adaptations with the AFDC mechanism . . . . . 88

5.3 .3 Optimal AFDC Parameters . . . . . . . . . . . . . . . . . . 90

5.3.4 Quantitative Comparison of the AFO and the AFDC mechanism 93

5.4 Discussion . . . . . . . . . . . . . . . . . . . . 94

6 Discussion and Future Work $\quad 97$

A Supplementary Calculations for the Cell Assembly Model 107

A.1 Normalization of the Network Model . . . . . . . . . . . . . . 107

A.2 Jacobi Matrix of the Population Model . . . . . . . . . . . . . . . . . 112

A.3 Maximum Input Amplitude for Cell Assembly Formation . . . . . . 113

B Frequency-Curve of the Van der Pol Oscillator $\quad 115$

$\begin{array}{lr}\text { Bibliography } & 117\end{array}$

$\begin{array}{ll}\text { Acknowledgements } & 133\end{array}$

$\begin{array}{ll}\text { Curriculum Vitae } & 135\end{array}$ 


\section{List of Figures}

1.1 Hypothesis and sub-hypotheses addressed in this thesis $\ldots \ldots$. . . 3

2.1 Structure of a neuron . . . . . . . . . . . . . . . 6

2.2 Synaptic transmission at a chemical synapse . . . . . . . . . . 7

2.3 Rate based neuron model and different firing rate functions . . . . . . 9

2.4 Taxonomy of the long-term memory system . . . . . . . . . . . . . 11

2.5 Phenomenological models of working memory . . . . . . . . . . . 13

2.6 Working memory n-back task . . . . . . . . . . . . . . . 14

3.1 Dynamics at the edge of chaos in random neuronal networks . . . . . . 23

3.2 Setup for testing a reservoir's performance in the n-back task . . . . . . 28

3.3 Training reservoir networks to perform the n-back task . . . . . . . 31

3.4 Influence of the interstimulus interval and of temporal unpredictability on the n-back performance of reservoir networks. . . . . . . . . . 32

3.5 Influence of different network parameters on the performance of reservoir networks in the n-back task with unpredictable stimulus timing . 34

3.6 Setup for the reservoir n-back task with additional readout units . . . . 36

3.7 Performance of reservoir networks with additional readout units in the $n$-back task with unpredictable stimulus timing . . . . . . . . . 37

3.8 Dynamics of a reservoir network during performing the n-back task projected onto the first two principal components . . . . . . . . 38

3.9 Performance of a reservoir network with additional readout units in n-back tasks with different response delays . . . . . . . . . . . . . 39

4.1 Network model for studying the self-organized formation of CAs . . . 45

4.2 Active neighbor ratio (ANR) as a measure of activity clustering . . . . 49

4.3 Self-organized formation of a CA . . . . . . . . . . . . . 52

4.4 Mean long-term development of a CA . . . . . . . . . . . . . . 53

4.5 Analytical derivation of the CA size and numerical results . . . . . . 55

4.6 Mean number of active recurrent and feedforward inputs for CA neurons 57

4.7 Patter completion and separation properties of the network model . . 59

4.8 Formation of a second self-organized CA in the network model . . . . 61

4.9 Plasticity of feedforward and recurrent synapses during the sequential formation of two CAs . . . . . . . . . . . . . . . . . . 62

4.10 Structure of the population model . . . . . . . . . . . . . . 64

4.11 Nullclines and equilibria of the population model . . . . . . . . . . . 67

4.12 Bifurcation diagram of the population model for varied input amplitude 68

4.13 The role of heterosynaptic plasticity in the self-organized assignment of multiple CAs . . . . . . . . . . . . . . . . . . 71

4.14 Summary of the synaptic weight changes underlying the formation and allocation of memory representations . . . . . . . . 72

5.1 Adaptation of two oscillators with AFO mechanism . . . . . . . . . . 81 
5.2 Quantitative measures of the quality of a frequency adaptation process 83

5.3 Influence of the coupling strength $\epsilon$ and the learning rate $\eta$ on the adaptation of oscillators with AFO mechanism . . . . . . . . . . 84

5.4 Adaptation quality measures in the frequency space for different parameter values of the adaptive frequency Hopf oscillator. . . . . . . . . 85

5.5 Average combined quality measure $\langle Q\rangle$ in the $\epsilon-\eta$-parameter space . . 86

5.6 Examples of frequency adaptation with the AFDC mechanism . . . . . 90

5.7 Adaptation quality measures for different parameter values of the Hopf oscillator with AFDC mechanism in the $v_{0}-v_{\text {ext }}$-frequency space . 91

5.8 Frequency space averaged quality index $\langle Q\rangle$ for different parameter values of oscillators with the AFDC mechanism . . . . . . . . . . 92

5.9 Average quality measures for the best found parameterizations of the oscillators with AFO mechanism and with AFDC mechanism . . . . 93

B.1 Limit cycle and frequency curve of the Van der Pol oscillator . . . . . 115

\section{List of Tables}

4.1 Overview of the network model parameters and their values . . . . . . 48

4.2 Overview of the population model parameters and their respective values. . . . . . . . . . . . . . . . . . 65 


\title{
List of Abbreviations
}

\author{
AFDC Adaptation through Fast Dynamical Coupling \\ AFO Adaptive Frequency Oscillator \\ ANR Active Neighbor Ratio \\ CA Cell Assembly \\ CPG Central Pattern Generator \\ ESN Echo State Network \\ FORCE First-Order Reduced and Controlled Error \\ LSM Liquid State Machine \\ LTM Long-Term Memory \\ PCA Principal Component Aanalysis \\ PFC Prefrontal Cortex \\ RMS Root Mean Square \\ RMSE Root Mean Squared Error \\ STP Short-Term Plasticity \\ WM Working Memory
}





\section{Chapter 1}

\section{Introduction}

Humans and animals expose a large variety of complex behaviors. Importantly, in order to survive in a continuously changing environment, a subject has to be able to adapt its behavior based on current stimuli and past experiences. The latter requires the neuronal systems, which control the behavior of a subject, to store information about past experiences, i.e., to build up memories. There are different types of memories which are built and maintained across various different time scales. The ability to store and to process information on short time scales from seconds up to a minute is referred to as working memory (WM; Baddeley and Hitch, 1974). Working memory plays an important role in mental reasoning, planing and, for instance, also calculating (Diamond, 2014). Despite of the central importance of WM, it is not clear which kind of dynamics underlies the neuronal system that implements WM.

Ultimately, all kinds of memory are related to the neuronal substrate by which they are implemented in the human or animal brain. This neuronal substrate consists of excitable nerve cells, the neurons, which exchange electrochemical signals via so called synapses (Kandel et al., 2000). WM is assumed to be implemented in the neuronal system of the prefrontal cortex (PFC) of the brain (Fuster and Alexander, 1971; Goldman-Rakic, 1995; Riley and Constantinidis, 2016). In many experiments, it is found that during storing of information in WM, specific neurons in the PFC encode the maintained information in form of persistent activity (Curtis and D'Esposito, 2003; Sreenivasan et al., 2014; Riley and Constantinidis, 2016). This implies that information in WM is represented by self-sustaining activity states, so called attractor states. Other studies, however, find pieces of evidence for a different mechanism to store information in WM. These experiments find that the information is represented in complex sequences of different activity patterns, so called transient trajectories (Jun et al., 2010; Hussar and Pasternak, 2012). Based on these observations, it is proposed that WM might rely on an attractor-less transient flow of activity triggered by the incoming stimuli (Stokes et al., 2013; Barak and Tsodyks, 2014). It is not clear which of these two seemingly contradictory types of neuronal dynamics, attractordominated dynamics and purely transient dynamics, actually underlies the neuronal implementation of WM.

In this thesis, we propose a possible solution to this problem: We evaluate the hypothesis that complex and robust WM operation is enabled by the interaction of transient neuronal dynamics with self-organized attractor states in other neuronal memory systems. Here, by complex WM operation, we refer to the ability to produce non-linear timedependent signals based on the information stored in WM, i.e., to perform complex computations. Robust WM operation, on the other hand, requires that both the storage of the information in WM as well as the ability to perform complex computations are stable against different kinds of noise in the stimuli received by the WM system, in particular temporal unpredictability. This hypothesis is based on the observation that WM does not operate in isolation but is continuously exchanging signals with 
other memory systems, for instance the long-term memory (LTM; Baddeley et al., 1988; Hulme et al., 1991; Ranganath et al., 2005; Poirier et al., 2011; Kamiński, 2017). Thus, even if the dynamics of the WM system does not explicitly rely on attractor states, it might still profit from such dynamical structures in other memory systems. More explicitly, we claim that the level of computational power and robustness observed in human WM can only be achieved when exploiting both the robustness of attractor dominated dynamics and the computational power of transient dynamics. Here, these attractor states may also be given by periodic attractors which allow to stably produce ongoing periodic signals as required, for instance, in the control of locomotion. Hence, we propose that the interaction between transient dynamics and attractor states enables the robust storage of information in WM as well as the generation of different long-lasting time-dependent output signals.

In order to verify the main hypothesis, we derive three necessary sub-hypothesis (Figure 1.1). After an overview of the relevant theoretical concepts and experimental results in Chapter 2, the sequential evaluation of these three sub-hypotheses determines the structure of this thesis.

The first sub-hypothesis states that WM requires both transient neuronal dynamics as well as distinct attractor states for robust information storage and complex computation. Note that this sub-hypothesis excludes the possibility that WM might rely solely on transient dynamics or solely on attractor states. It does not require, however, that these attractor states are actually part of the systems mainly implementing WM. To evaluate this sub-hypothesis, in Chapter 3, we study a recurrent network model which relies on transient dynamics (Jaeger, 2001; Maass et al., 2002; Sussillo and Abbott, 2009). In particular, we investigate the robustness of the performance of this model in solving an established WM task (Kirchner, 1958; Baddeley, 2003) with respect to unpredictable stimulus timing. We compare this robustness with the level of robustness observed for human subjects performing the same WM task (Koppe et al., 2014). We find that the purely transient network model is significantly more vulnerable to unpredictable stimulus timing than a human subject. Based on this finding, we propose a method to overcome this qualitative difference and show that this method is effectively introducing additional attractor states into the network dynamics. Based on further analyses, we propose a functional separation of the dynamics underlying WM: Transient dynamics allow the production of complex output signals and attractor states are responsible for the robust storage of information.

According to our main hypothesis, the attractor states enabling robust WM operation are formed in a self-organized way in other memory systems. The mechanism underlying the formation of these attractor states are defined by our second subhypothesis. It states that attractor states may be formed and assigned in a self-organized way by the interplay of two synaptic plasticity processes, namely Hebbian plasticity and synaptic scaling, in the LTM system. Synaptic plasticity refers to activity-dependent modifications of the transmission efficacies of synapses in a neuronal network (Abbott and Nelson, 2000). It is assumed that neuronal networks store long-term memories by ways of synaptic plasticity processes (Martin et al., 2000; Takeuchi et al., 2013). Two special forms of synaptic plasticity are Hebbian plasticity (Hebb, 1949) and synaptic scaling (Turrigiano, 2008). Their interaction has been shown to enable the self-organized emergence of attractor states in recurrent networks (Tetzlaff et al., 2013). These attractor states emerge due to the formation of Hebbian cell assemblies (CAs; Hebb, 1949) in the network. A CA is given by a strongly interconnected group of neurons that tend to fire simultaneously. Up to now, there is no model available that explains how these attractor states may be simultaneously formed and also correctly assigned to different stimuli. In Chapter 4, we derive such a model. We 


\section{Hypothesis}

Complex and robust WM operation is enabled by the interaction of transient neuronal

dynamics with self-organized attractor states in other neuronal memory systems.
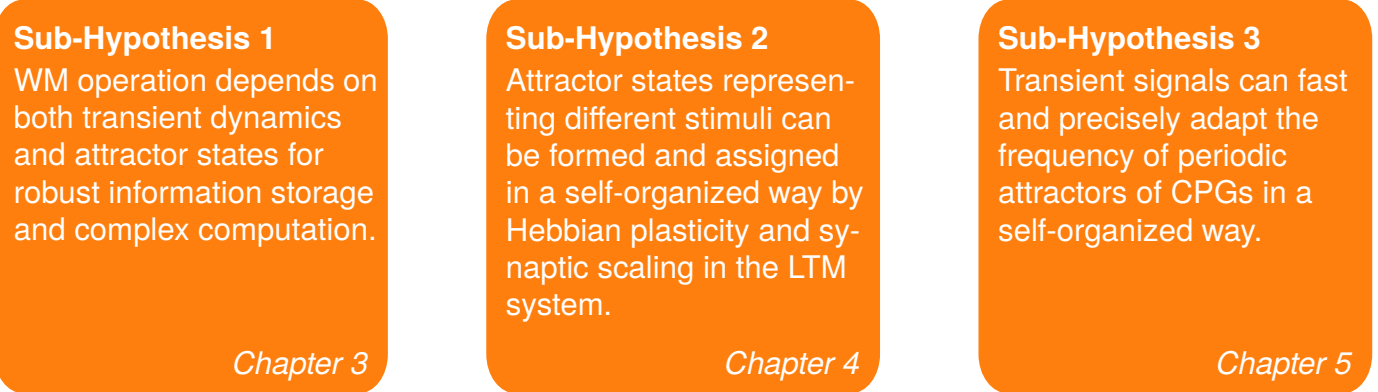

Figure 1.1: Hypothesis and sub-hypotheses addressed in this thesis. From the main hypothesis of this thesis (blue), we derive three necessary sub-hypotheses (orange). Every sub-hypothesis is evaluated in a dedicated chapter. The abbreviations denote the working memory (WM) and the long-term memory (LTM) and central pattern generators (CPGs). See the main text for details.

show that these CAs constitute input dependent attractors of the network dynamics and thus verify the second sub-hypothesis.

Given that our first sub-hypothesis states that complex computations in WM depend on transient dynamics, the question arises how these rather short-lasting transient dynamics may be used to produce long-lasting neuronal signals. In particular, we investigate the case of long-lasting periodic output signals with different frequencies as they are required, for instance, in the control of locomotion. Our third sub-hypothesis states that transient signals can fast and precisely adapt the frequency of periodic attractors of central pattern generators (CPGs) in a self-organized way. CPGs are specific neuronal circuits which are able to produce periodic neuronal signals without the requirement for any periodic input signal (Hooper, 2001; Ijspeert, 2008). The periodic neuronal signal emerges due to a periodic attractor within these circuits. Thus, in an abstract sense, CPGs are nonlinear oscillatory systems or simply oscillators. If such an oscillator is supposed to transform a transient periodic input signal into a long-lasting periodic output signal with the same frequency, there needs to be a mechanism which allows the oscillator to adapt its intrinsic oscillation frequency in a self-organized way. While such a mechanism has been developed (Righetti et al., 2006), in Chapter 5, we show that this mechanism suffers from a frequency-specific trade-off between fast adaptation and precise adaptation. Therefore, we develop a new adaptation mechanism which allows for fast and precise adaptations within a wide range of oscillation frequencies. This new mechanism provides a possible explanation for the processes that allow to transform short-lasting transient signals from the WM system into long-lasting time-dependent motor control signals.

After evaluating the different sub-hypotheses, in Chapter 6, we discuss our results in the context of our main hypothesis. We evaluate in how far this hypothesis could be validated by this thesis and explain what remains to be done for a final evaluation. Finally, we list possible future work and describe an experimental setup to test a behavioral prediction arising from our theoretical results. 



\section{Chapter 2}

\section{Experimental and Theoretical Background}

The ability to store information about past experiences and events, i.e., to form memories, is an indispensable presupposition for adaptive and intelligent behavior of an individual. It allows to transfer acquired knowledge of the past to future situations and to react accordingly. Ultimately, all kinds of memory are related to the neuronal systems that underly behavioral control in humans and animals in the brain (Section 2.1). The basic ideas of how these neuronal systems are able to build up memory date back to Hebb, (1949) and are summarized as the Hebbian postulates (Section 2.2). Actually, the term memory refers to different phenomena occurring on very different time scales and involving different neuronal systems (Nadel and Hardt, 2011). On the one hand, animals and humans are able to store information that is maintained for very long time scales ranging from days over years to life-long. This kind of memory is referred to as long-term memory (LTM, Section 2.3). On the other hand, there is working memory (WM) which allows to store information of recent events and sensory inputs on short-time scales and to manipulate and process this stored information (Section 2.4). Apart from these two main types of memory, there are also other mechanism in the human brain which employ different types of memory. For instance, there are mechanisms which allow specific neuronal circuits to produce long-lasting rhythmic output signals at a certain memorized frequency (Section 2.5). In the following, we summarize the experimental and theoretical foundations of these concepts and mechanisms.

\subsection{Neuronal Networks of the Brain}

The brain is the center of the nervous system in humans and most animals. It comprises a large number of electrically excitable cells which are called neurons. These neurons exchange electrochemical signals with other neurons at specific locations called synapses. Thus, the neurons form an interacting neuronal network. Every synapse in this network is characterized by a certain transmission efficacy. Importantly, these efficacies may be adapted, for instance, based on neuronal activity. This phenomenon is referred to as synaptic plasticity. It is widely assumed that synaptic plasticity processes are the cellular basis of learning and memory in the brain (Martin et al., 2000).

\section{Neurons}

While their is a vast amount of different kinds of neurons, most of them share a basic common structure (Figure 2.1; Kandel et al., 2000). This structure is composed of the 


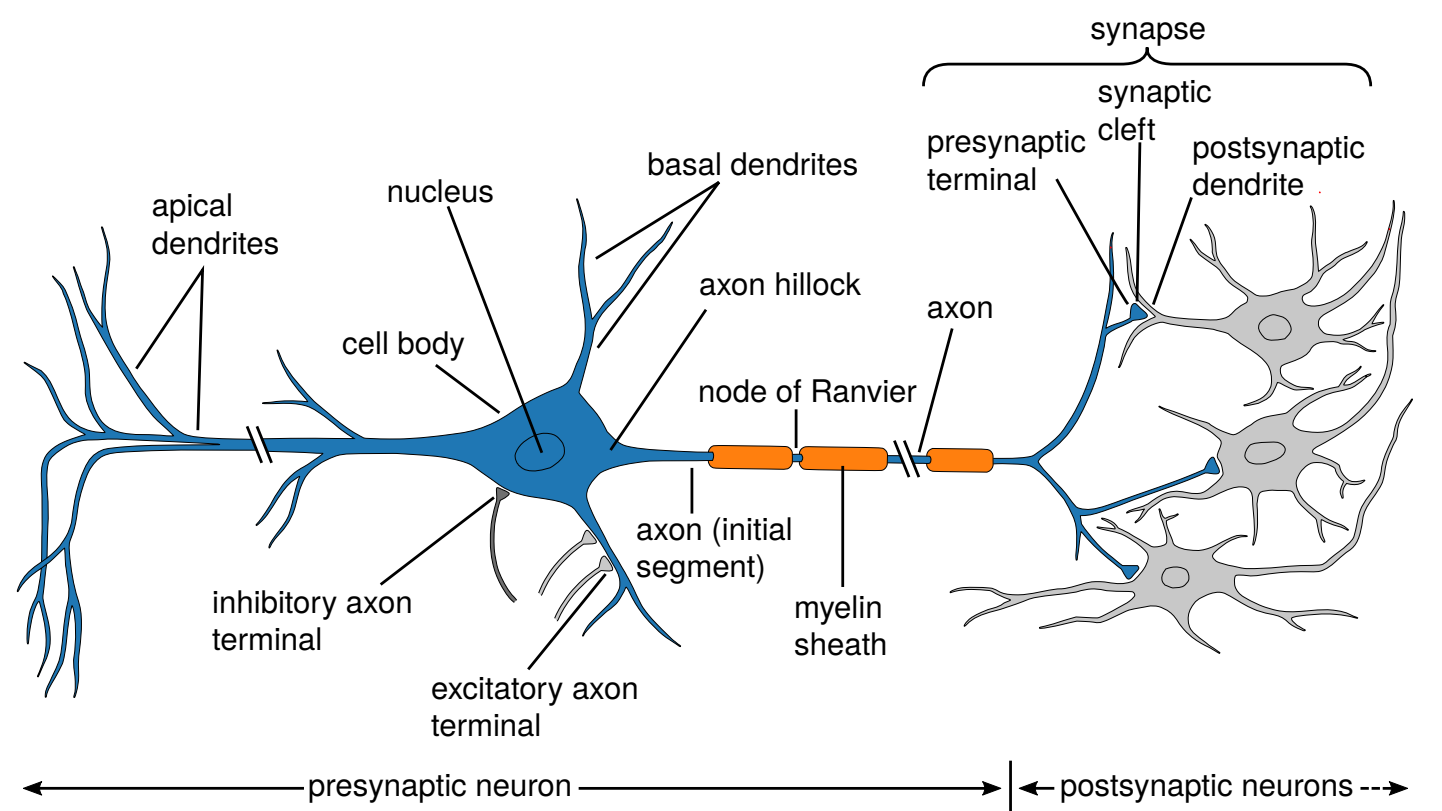

Figure 2.1: Structure of a neuron. Most neurons in the vertebrate neuronal system share the basic structure depicted here. A neuron receives signals via synaptic contacts either directly at its cell body, which contains the nucleus, or the dendrites. Apical dendrites emerge at the apex of the cell body, basal dendrites directly from the cell body. Both types of dendrites transmit the synaptic signals to the cell body where they modify the membrane potential of the cell. If this potential crosses a certain threshold, an action potential is generated at the axon hillock and transmitted along the axon. The axon is insulated from the surrounding environment by a myelin sheath. This sheath has regular gaps, the so called nodes of Ranvier at which the traveling action potential is regenerated. The axon terminals form synapses with the dendrites or cell bodies of other neurons. At a chemical synapse as depicted here, the presynaptic neuron transmits signals to the postsynaptic neuron by releasing neurotransmitters into the synaptic cleft. Depending on the type of neurotransmitter, these signal may either increase the membrane potential within the postsynaptic neuron (excitatory synapse) or decrease it (inhibitory synapse). Figure adapted with permission from E. R. Kandel, J. H. Schwartz, and T. M. Jessel: Principles of Neural Science (C) 2000 McGraw-Hill Education.

cell body, which contains the nucleus, the dendrites and the axon. The dendrites are tree-like extensions which originate from the cell body. At various locations of the dendrites, there are synapses via which the neuron receives electrochemical signals from other neurons. The dendrites integrate these signals and transmit them to the cell body. A neuron may also receive synaptic signals directly at the cell body. In either case, the integrated signals influence the membrane potential of the cell, i.e., the voltage difference between the inside of the cell and its outside environment. Once the membrane potential of the cell reaches a certain threshold, a short rapid rise and decay of the membrane potential is initialized at the axon hillock of the cell body (Stuart et al., 1997). These stereotypic peaks of the membrane potential are called action potentials or spikes (Barnett and Larkman, 2007). They are generated by the opening of voltage dependent ion channels in the cell membrane. A generated action potential propagates along the axon away from the cell body. The axon is a long and thin projection of the neuron which originates at the axon hillock. Most axons are covered by sheaths of myelin which insulate the axon from its environment. At regular spatial intervals, the myelin sheath is interrupted by so called nodes of Ranvier which support the transmission of the signal along the axon (Kandel et al., 2000). At the end of the axon are the axon terminals. These terminals form synapses with the dendrites or cell bodies of other neurons. 
action potential in axon terminal opens $\mathrm{CA}^{2+}$ channels
$\mathrm{CA}^{2+}$ entry causes vesicle fusion and transmitter release receptor channels open,

$\mathrm{Na}^{+}$enters the postsynaptic cell and vesicles recycle

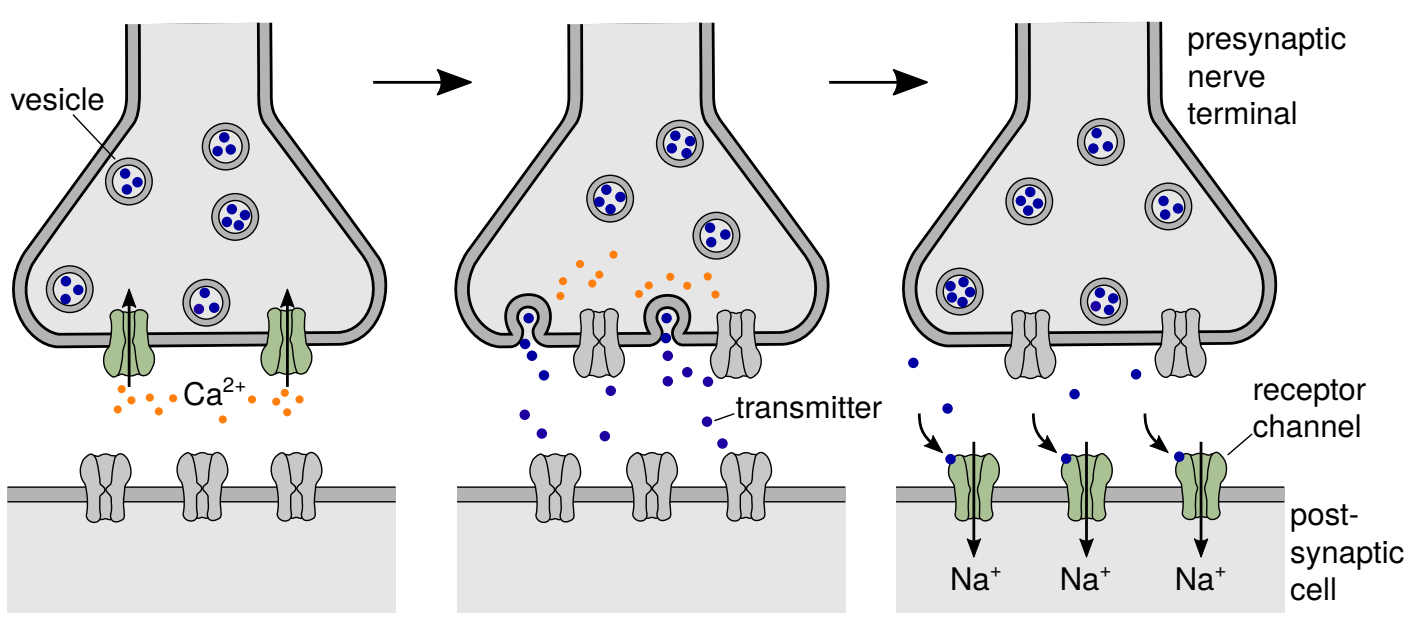

Figure 2.2: Synaptic transmission at a chemical synapse. The transmission of electrochemical signals at a chemical synapse is initiated when an action potential arrives at a presynaptic terminal of an axon. Due to the increased membrane potential, voltage dependent $\mathrm{Ca}^{2+}$ channels open and $\mathrm{Ca}^{2+}$ ions enter the cell. This causes vesicles containing neurotransmitters to fuse with the membrane and thereby release the neurotransmitter into the synaptic cleft. The neurotransmitter molecules bind to receptors in the postsynaptic membrane which causes different ion channels of the postsynaptic membrane to open (or close). For instance, these open channels may allow $\mathrm{Na}^{+}$to enter the postsynaptic cell which results in a change of the respective membrane potential. Figure adapted with permission from E. R. Kandel, J. H. Schwartz, and T. M. Jessel: Principles of Neural Science (c) 2000 McGraw-Hill Education.

\section{Synapses}

At a synapse, the membrane potential of one neuron may influence the membrane potential of a second neuron. There are two qualitatively different types of synapses: electrical synapses and chemical synapses. At an electrical synapse, the membranes of two neurons approach each other close enough to form so called gap junctions. Gap junctions are channels which allow the bidirectional passage of electrical currents and small molecules (Bennett and Zukin, 2004; Pereda, 2014). Thus, at an electrical synapse, two neurons are directly electrically coupled. This allows bidirectional signal transmission without any significant synaptic delay. At the same time, electrical synapses cannot amplify or transform the transmitted signals (Pereda, 2014). This is possible in chemical synapses. At a chemical synapse, a presynaptic axon terminal is separated from the postsynaptic dendrite by the synaptic cleft (Figure 2.2). Action potentials arriving at the axon terminal cause the release of specific substances called neurotransmitters into the synaptic cleft (Kandel et al., 2000). The neurotransmitters diffuse through the synaptic cleft and bind to receptors in the postsynaptic membrane. This leads to the opening of channels in the postsynaptic membrane which allows different kinds of ions to flow into or out of the cell. These ion currents alter the membrane potential of the postsynaptic neuron. Thus, the action potential arriving at the presynaptic neuron induces an electrical signal at the postsynaptic neuron. This signal is called a postsynaptic potential. Depending on the type of neurotransmitter released by the presynaptic terminal and the type of receptors in the postsynaptic membrane, a postsynaptic potential may either increase or decrease the membrane potential of the postsynaptic neuron. Synapses which mediate an increase of the postsynaptic membrane potential are called excitatory synapses (Chua et al., 2010). 
Synapses which transmit signals that decrease the postsynaptic potential are called inhibitory synapses (Kubota et al., 2016). According to Dale's principle, a neuron releases the same neurotransmitter substances at all of its axonal terminals (Strata and Harvey, 1999). We can therefore classify neurons as either excitatory neurons or inhibitory neurons.

The amplitude of a postsynaptic potential evoked by a synaptic transmission depends on a number of synapse specific parameters. These include, for instance, the number of available vesicles in the presynaptic terminal, the probability of neurotransmitter release and the number of receptors and ion channels in the postsynaptic membrane (Markram et al., 1998). All these influences determine the transmission efficacy of a synapse or, in other words, the synaptic weight (Dayan and Abbott, 2001). A larger synaptic weight denotes a stronger influence of the presynaptic neuron on the membrane potential of the postsynaptic neuron. Importantly, the weight of a given synapse is not fixed but can be adapted over time by different processes summarized as synaptic plasticity.

\section{Synaptic Plasticity}

There is a broad variety of different types of synaptic plasticity processes which adapt different properties of the presynaptic or the postsynaptic neuron. In general, an increase of the synaptic weight is called potentiation. A weakening of the synaptic weight is called depression. A particular well-studied class of plasticity processes is subsumed as Hebbian plasticity (Hebb, 1949). Hebbian plasticity is also referred to as homosynaptic plasticity and describes synaptic plasticity processes that act associative and input-specific (Abbott and Nelson, 2000). This means, Hebbian plasticity strengthens synapses that transmit signals in between neurons which simultaneously produce action potentials. Hebbian plasticity is mostly observed in long-term potentiation processes that last for hours, days or possibly even longer (Malenka and Bear, 2004; Nicoll and Roche, 2013). Long-term potentiation is evoked by neurotransmitters which are released from the presynaptic axon terminal and bind to specific receptor channels in the postsynaptic membrane and thereby open them. If the postsynaptic membrane potential is large enough, these open channels allow the influx of calcium ions. The increased calcium concentration in the postsynaptic neuron leads to both an increase of the number of neurotransmitter receptors in the postsynaptic membrane as well as to an enhanced sensitivity of the existing receptors (Lüscher and Malenka, 2012). Both effects result in an increase of the synaptic weight. A larger synaptic weight, in turn, increases the influence of the presynaptic neuron on the membrane potential of the postsynaptic neuron. Thus, it also increases the probability that an action potential in the presynaptic neuron evokes an action potential in the postsynaptic neuron which leads to further Hebbian plasticity. Therefore, Hebbian plasticity results in a positive feedback loop (Abbott and Nelson, 2000). This feedback loop is broken by another class of plasticity process called homeostatic plasticity (Turrigiano and Nelson, 2004). Homeostatic plasticity refers to plasticity processes that act in order to stabilize certain neuronal parameters at a given set point. A specific form of homeostatic plasticity is the process of synaptic scaling (Turrigiano, 2008). Synaptic scaling adjusts the weights of all synapses via which a neurons receives signals from other neurons to maintain a certain target rate of action potentials. Therefore, synaptic scaling is a plasticity process which is not input-specific and referred to as heterosynaptic plasticity (Chistiakova et al., 2014). 
a

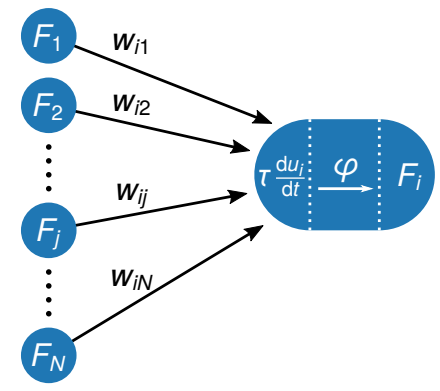

b

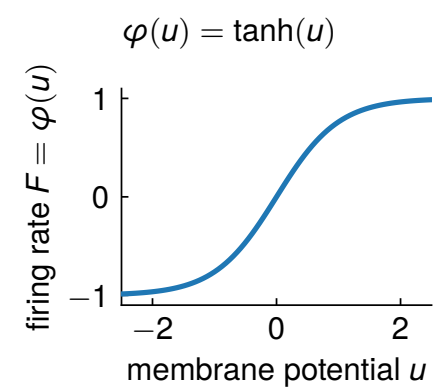

C

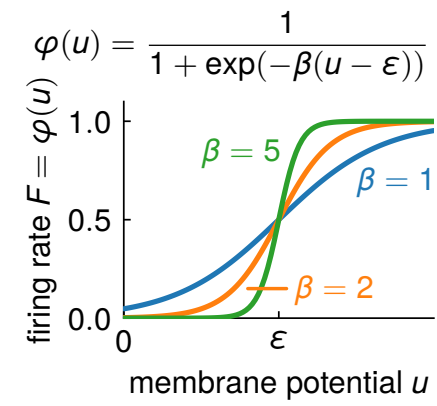

Figure 2.3: Rate based neuron model and different firing rate functions. (a) In the rate based description of neuronal networks, every neuron $i$ is described by the dynamics of a (leaky) membrane potential $u_{i}, i \in\{1, \ldots, N\}$. The firing rate $F_{i}$ of a neuron is given by a firing rate function $\phi$ of the membrane potential $u_{i}$. Synaptic interactions are modeled by linearly coupling the firing rates $F_{j}, j \in\{1, \ldots, N\}$, of other neurons in the network to the dynamics of $u_{i}$. The coupling strength $w_{i j}$ represents the synaptic weight. (b) A possible choice of the firing rate function $\phi$ is given by the hyperbolic tangent which produces firing rates in between -1 and 1 (used in Chapter 3 ). (c) A second class of firing rate functions is given by the logistic function with an inflection point at $u=\epsilon$ and a steepness $\beta$ which produces strictly positive firing rates between 0 and 1 (used in Chapter 4).

\section{Rate Based Models of Neuronal Networks}

The functioning of neurons, synapses and synaptic plasticity is based on complex biophysical processes involving different kinds of proteins, neurotransmitters, and ions. Depending on the specific research question, it may not be necessary to model all of these details when studying the dynamics of neuronal networks. Therefore, neuron models on very different levels of abstraction have been developed. They range from the detailed electrophysiological Hodgin-Huxley-Model (Hodgkin and Huxley, 1952) over the simplified integrate-and-fire neuron model (Burkitt, 2006) up to the very reduced McCulloch-Pitts-neuron (McCulloch and Pitts, 1943). In this thesis, we use an intermediate level of description, namely a rate-based neuron model (Dayan and Abbott, 2001; Shriki et al., 2003). At this level of description, the timing of the individual action potentials of a neuron is disregarded. Instead, neurons are described as point-like units whose current state is determined by a leaky membrane potential (Figure 2.3 a). The membrane potential of a neuron determines the current rate of action potentials produced by this neuron. This rate is called the firing rate of the neuron. It is given by a sigmoidal function of the the membrane potential (Figure $2.3 \mathrm{~b}$ and Figure $2.3 \mathrm{c}$ ). Alternatively, the firing rate may also be interpreted as the current firing probability of the neuron (Gerstner et al., 2014).

Synaptic interactions are modeled by coupling the presynaptic firing rate linearly to the dynamics of the membrane potential of the postsynaptic neuron. The strength of this coupling corresponds to the synaptic weight. Thus, synaptic plasticity is modeled by modifying this coupling strength based on the neuronal activity.

\subsection{The Hebbian Postulates}

According to the synaptic plasticity and memory hypothesis, the neuronal networks in the brain store memories about past experiences by alterations of synaptic weights, i.e, by synaptic plasticity (Takeuchi et al., 2013). This notion dates back to the influential book "The Organization of Behavior" (Hebb, 1949). In this book, Donald 
Hebb constructs a theory about the neuronal mechanisms which underly behavioral learning and memory. This theory is based on three postulates.

The first postulate states that "when an axon of cell A is near enough to excite a cell $\mathrm{B}$ and repeatedly or persistently takes part in firing it, some growth process or metabolic change takes place in one or both cells such that A's efficiency, as one of the cells firing B, is increased" (Hebb, 1949). In other words, correlated activity of the presynaptic and the postsynaptic neurons leads to an increase of the weight of the respective synapse. This is often subsumed in the statement "What fires together wires together." (Shatz, 1992). In the field of computational neuroscience, this proposal is referred to as the Hebb rule. As discussed above, Hebbian plasticity refers to synaptic plasticity processes that modify synapses according to this rule.

The second postulate builds upon the first one. It states that due to the first postulate, groups of cells which are repeatedly active simultaneously get associated by increased synaptic weights and form strongly interconnected cell assemblies (CAs) (Hebb, 1949). These CAs are considered to be the fundamental building block of LTM. They are able to memorize patterns of input-evoked activity and, due to their auto-associative nature, to restore these patterns later on also for incomplete input, i.e., to perform pattern completion. In addition, the recurrent excitation within a CA allows the firing of the respective neurons to persist even after the removal of the stimulus which originally evoked this activity. This represents a possible mechanism for information maintenance in WM.

In his third postulate, Hebb proposes the sequential activation of several CAs as the process which underlies thinking. The activation of one CA might project excitatory signals to another assembly and herewith evoke the activation of this second CA. By the same mechanism, this second CA might evoke a third one and so forth. Hebb termed this sequential activation of multiple CAs a phase sequence. According to his proposal, a phase sequence represents the flow of thoughts in a neuronal system (Hebb, 1949).

In particular the first and the second postulate are the basis of many current models of memory in the brain (Lansner, 2009). The ability for pattern completion of a CA is recognized as being essential for memory recall in LTM (Nadel and Hardt, 2011). Still, the formation and the temporal evolution of CAs in LTM are not completely understood (Buzsáki, 2010).

\subsection{Long-Term Memory}

Long-term memory (LTM) refers to the ability of the brain to store information for hours, days or even years. In general, there seems to be no relevant capacity limit for the information stored in LTM (Nadel and Hardt, 2011). The LTM can be separated into declarative and non-declarative memory (Figure 2.4). Loosely speaking, declarative memory encodes information about the "knowing that" while non-declarative memory encodes information about the "knowing how" (Ryle, 1949). The declarative memory is consciously accessible (Squire, 2004; Wood et al., 2011). It can be further subdivided into semantic memory and episodic memory. Semantic memory stores information about facts, ideas and concepts. Episodic memory, in turn, provides the ability to re-experience events in its original context (Tulving, 1983). In contrast to declarative memory, information stored in the non-declarative part of LTM is not directly consciously available. Furthermore, non-declarative LTM does not encode the characteristics of a single type of input stimuli but rather generalizes across multiple input sets and encodes their common features (Squire, 2004; Wood et 


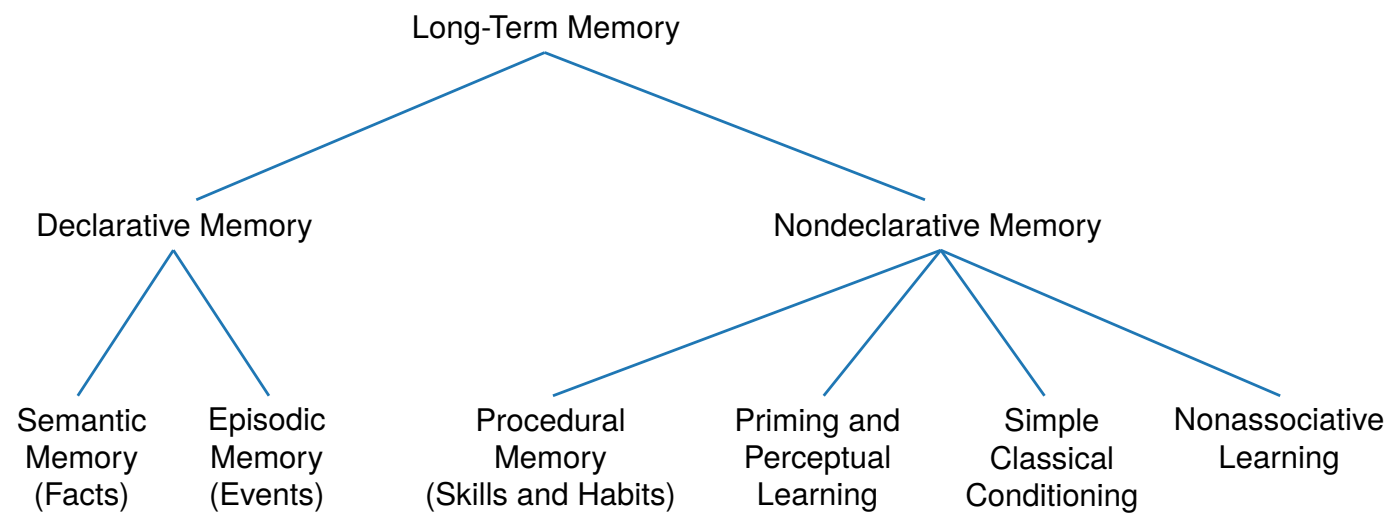

Figure 2.4: Taxonomy of the long-term memory system. The long-term memory contains the consciously available declarative memory and the nondeclaratative memory which is not directly consciously available. The declarative memory contains information about facts, concepts and events. The nondeclaratative memory contains learned skills and habits and other implicit types of learning and memory. Figure adapted from Squire, (2004).

al., 2011). The most prominent component of non-declarative memory is procedural memory which contains memories of skilled behavior and habits (Wood et al., 2011). Further non-declarative LTM components include priming and perceptual learning, simple classical conditioning and non-associative learning (Figure 2.4).

\subsubsection{Neural Correlates of Long-Term Memory}

The neuronal systems implementing the different types of LTM are distributed over the brain (Squire, 2004). In particular, the declarative and the non-declarative parts of LTM are implemented in distinct brain areas. Amnesic patients which cannot form new declarative memories can still acquire new procedural skills (Cohen and Squire, 1980). Declarative memory depends on an interplay of the hippocampus, the surrounding structures in the medial temporal lobe and the neocortex (Eichenbaum, 2000). New memories are formed in the hippocampus and later on consolidated and transfered to the neocortex (Wiltgen et al., 2004; Nadel and Hardt, 2011; Genzel et al., 2017). Procedural memory, in turn, is implemented by the basal ganglia and the cerebellum (Eichenbaum, 2000; Wolpert et al., 2001).

Recent technological advances and the resulting data from neuroanatomical and physiological studies lead to increasing support for the hypothesis that CAs are the fundamental building blocks of the neuronal systems implementing LTM (Palm et al., 2014; Holtmaat and Caroni, 2016). Furthermore, the synaptic connectivity in the cortex has been show to provide properties which are optimal for robustly storing a large number of attractor states like they are imposed by CAs (Brunel, 2016). While a lot of experimental results support the necessity of synaptic plasticity for the formation of memory, it is not clear whether synaptic plasticity alone is also sufficient for successful learning and recall (Martin et al., 2000). For instance, structural plasticity has been proposed to be necessary for long-term maintenance of information in memory (Chklovskii et al., 2004; Fauth et al., 2015).

\subsubsection{Neurocomputational Models of Long-Term Memory}

One of the earliest functional network models of LTM is the class of Hopfield networks (Hopfield, 1982; Hopfield, 1984). In a Hopfield network, a certain number of neurons is fully recurrently connected. The information which is stored in such a network is 
given by a number of different binary activation patterns. Every activation pattern is described by a fixed set of neurons which participate in firing in this pattern. The synaptic weights in between the different neurons are adjusted based on the first Hebbian postulate. Thus, the strength of the synapse in between two neurons depends on the number of stored patterns in which these two neurons expose the same activity state. As a result, the stored activation patterns become attractor states of the network dynamics. If the network is initialized in a state close to one of the stored patterns, the system dynamics converges towards the stored pattern. This is interpreted as a recall of the corresponding information from memory.

Most models of LTM rely on similar types of attractor dynamics as observed in the Hopfield model (Wood et al., 2011). Differences arise in the level of biophysical details that are included into the model and in the way in which the synaptic weights are adapted to form the different attractor states. In the Hopfield model, these weights are optimized and then applied as constant weights. Alternatively, a variety of correlation-based Hebbian learning rules have been proposed which adapt the synaptic weights during the actual operation of the network (Gerstner and Kistler, 2002). One particular example is a learning rule which adapts the synaptic weights based on the interplay between Hebbian plasticity and synaptic scaling (Tetzlaff et al., 2011). This learning rule has been shown to form stable CAs in a recurrent network which constitute input dependent attractor states of the network activity dynamics (Tetzlaff et al., 2013). Thus, once formed, a CA in this model allows the fast recall of earlier experienced patterns. Furthermore, this model is able to explain nontrivial reconsolidation effects (Tetzlaff et al., 2013).

\subsection{Working Memory}

Working memory (WM) refers to the ability to store memories that persist for several seconds up to minutes (Baddeley, 2012; Constantinidis and Klingberg, 2016). The duration during which an item is kept in WM can be increased by active rehearsal methods (Brown, 1958; Peterson and Peterson, 1959; Nadel and Hardt, 2011). In addition to maintenance of the information, WM also enables the manipulation of the memory content (Cowan, 2008; Nadel and Hardt, 2011; Baddeley, 2012). For instance, during reading a text, $\mathrm{WM}$ allows to memorize the recently perceived words and to connect them internally to a sentence. In contrast to LTM, WM is characterized by a limited capacity (Cowan, 2001). The number of items that can be kept simultaneously active in WM lies between four and seven (Miller, 1956; Cowan, 2001). It can be increased by chunking techniques, i.e., by hierarchically combining individual items to meaningful "chunks" (Miller, 1956). Differences in WM capacity have been shown to correlate with variations in different abilities and skills, for instance control of attention (Kane et al., 2007a), the ability for non-verbal reasoning (Kyllonen and Christal, 1990) and even academic performance (Gathercole et al., 2003).

The idea that LTM and WM actually represent two distinct neuronal systems dates back to James, (1890). Today, there is a lot of phenomenological evidence for this notion. For instance, there are amnesic patients who show normal performance in WM tasks but achieve only significantly reduced performance in tasks requiring LTM (Jeneson and Squire, 2011). This includes the popular case of the patient H.M. which lost his ability to form new memories in LTM after the surgical removal of his hippocampus (Squire, 2009). The fact that he was able to acquire memories on short time scales but not on longer time scales supports the view that separate neuronal systems are responsible for WM and LTM (Nadel and Hardt, 2011). Still, 


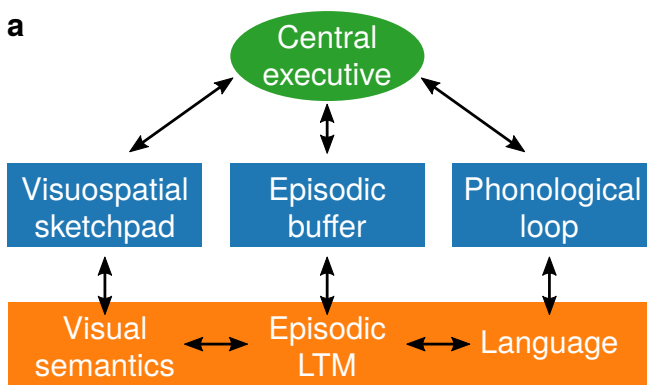

b

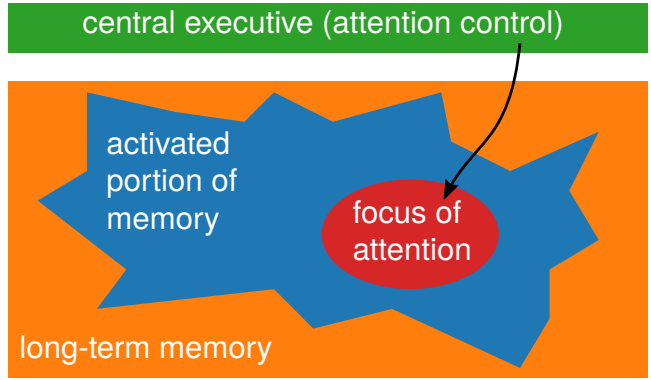

Figure 2.5: Phenomenological models of working memory. (a) According to the influential model of Baddeley and Hitch, (1974), which was later extend by Baddeley, (2000), WM consists of four components: A central executive (green) and three different short-term storage systems (blue). These short-term storage systems interact with the related information storage systems in LTM (orange). Figure adapted from Baddeley, (2000). (b) Alternatively to viewing WM and LTM as two distinct systems, Cowan, (1988) proposes that WM is given by an activated portion (blue) of the information stored in LTM (orange). Only the part of this activated information which is currently in the focus of attention (red) is consciously accessible. This focus of attention is controlled by a central executive (green). Figure adapted from Cowan, (2008).

WM continuously interacts with LTM. For instance, WM is required for forming new representations in LTM (Baddeley et al., 1988). At the same time, existing LTM representations support the processes in WM. The number of words that can be remembered and recalled in correct order in a WM task is larger for familiar words than for unfamiliar ones (Hulme et al., 1991). In addition, the number of remembered words is also higher if these words belong to the same semantic category (Poirier et al., 2011). Thus, information which can be related to existing representations in LTM is easier to maintain in WM. Furthermore, LTM supports WM when the capacity of WM is exceeded or when the attention of the subject is distracted (Jeneson and Squire, 2011).

The most influential phenomenological model of WM has been formulated by Baddeley and Hitch, (1974) and later on revised by Baddeley, (2000). According to the revised model, WM consists of four distinct components (Figure $2.5 \mathrm{a}$ ). The central executive is responsible for the overall control of the cognitive processes in WM. It has access to three distinct short-term storages named the phonological loop, the visuo-spatial sketchpad and the episodic buffer. The phonological loop stores auditory memory components and implements auditory rehearsal mechanisms. The visuo-spatial sketchpad keeps visual stimuli active as mental images. The episodic buffer stores chronological sequences of information from different domains. All of these three subsystems also interact with the related parts of LTM. Alternatively to viewing WM and LTM as two different storage systems, Cowan, (1988) proposed the embedded process model (Figure $2.5 \mathrm{~b}$ ). In this model, the information currently stored in WM is given by the information in LTM which is temporary in a state of higher activation. Only a subset of this activated information is also contained in the focus of attention. The focus of attention contains the information that the subject is consciously aware of and is controlled by a central executive component. Thus, from a psychological perspective, it is not clear whether WM and LTM constitute two manifestations of the same underlying neuronal system or whether they are implemented by two distinct systems. 
a

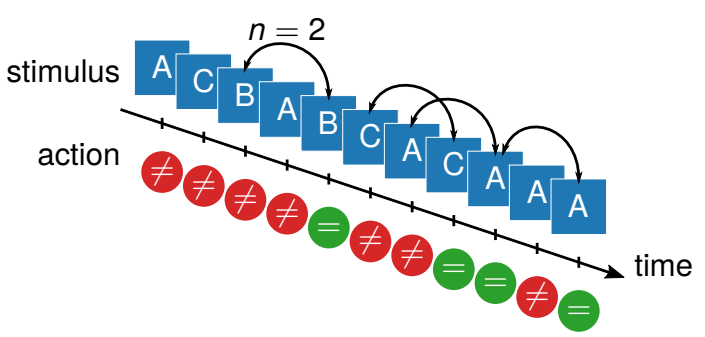

b

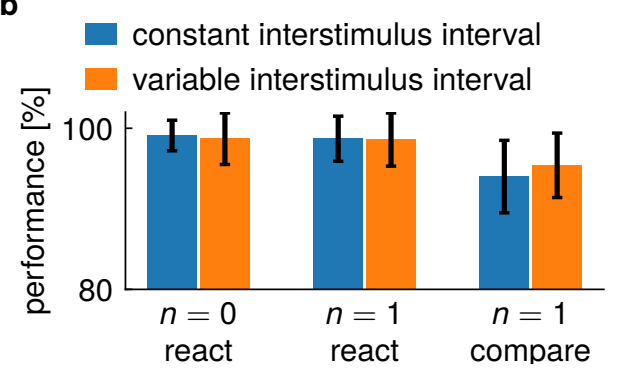

Figure 2.6: Working memory n-back task. (a) During an n-back task, a subject is exposed to a series of incoming stimuli. Typically, these stimuli are presented either visually or verbally. Whenever a new stimulus arrives, the subject has to decide whether this stimulus equals the one seen $n$ steps before (here $n=2$ ) and has to perform an appropriate action. (b) The performance of subjects performing the n-back task does not depend on whether the input stimuli are presented with constant interstimulus intervals (predictable temporal structure) or with variable interstimulus intervals (unpredictable stimulus structure). This has been verified for $n=0$ and $n=1$ and the original n-back task ("compare") and a variant in which the target action of the subject only depends on the stimulus received $\mathrm{n}$ steps before ("react"). The stimuli consist of squares and triangles. The constant interstimulus interval is $4 \mathrm{~s}$. The variable interstimulus intervals vary between $2.5 \mathrm{~s}$ and $5.5 \mathrm{~s}$. Data extracted from Koppe et al., (2014).

\subsubsection{The N-Back Task as a Measure of Working Memory}

In order to study the properties of WM, different experimental tasks for human or animal subjects have been developed. One of these experimental tasks is the so-called n-back task (Kirchner, 1958). The n-back task is an established experimental paradigm to explore different features of WM in humans and animals (Baddeley, 2003; Conway et al., 2005; Jaeggi et al., 2010).

During performing an n-back task, the subject is exposed to a series of sequentially incoming stimuli (Figure 2.6a). On every arrival of a new stimulus, the subject has to detect whether this new stimulus equals the stimulus presented $n$ steps before. Based on the result of this comparison, the subject has to perform a beforehand defined action. For instance, the subject might have to push a specific button whenever the current stimulus and the stimulus observed $n$ steps before are identical. Typically used values of $n$ range between 1 and 3. The different stimuli may be presented verbally, for instance in the form of words, or nonverbally, for instance in the form of different visual inputs. The performance of a subject in solving the n-back task is measured by the ratio of correctly performed actions.

The n-back task requires the subject to (i) perceive and process the incoming stimuli, (ii) store the relevant information about the stimuli, (iii) update and overwrite the stored information at every new stimulus, and (iv) manipulate this information to compare the current and the past stimuli. It therefore covers many of the key components of working memory (Owen et al., 2005). In addition to comparing WM performance of different subjects, the n-back task is also employed as a training task to improve WM performance of individual subjects (Jaeggi et al., 2008). However, there are also studies that challenge the validity of the n-back task as a measure of WM by demonstrating little correlation with other WM measures, for instance WM span (Kane et al., 2007b). In the latter, the subject has to remember items while performing a secondary cognitive task. However, these little correlations might also emerge from the fact that the n-back task and the WM span task capture different aspects of WM operation (Kane et al., 2007b).

The individual stimuli in an n-back task are separated by specific time intervals. We refer to these time intervals as the interstimulus intervals. If all interstimulus 
intervals are identical, the n-back task has a predictable temporal structure. If, in contrast, the interstimulus intervals are randomly drawn from a certain distribution, the temporal structure is unpredictable. Subjects performing the n-back task with unpredictable temporal structure show an increased activation in several brain regions as compared to subjects performing the n-back task with predictable stimulus timing (Koppe et al., 2014). Furthermore, an increase of the reaction time is observed in the case of unpredictable stimulus timing. Interestingly, however, introducing temporal unpredictability in the n-back task does not inhibit the level of performance of the subjects (Figure 2.6b). This demonstrates the robustness of WM performance with respect to unpredictable stimulus timing. We can use this finding to evaluate neurocomputational models of WM by testing whether they achieve the same level of robustness with respect to unpredictable temporal structure.

\subsubsection{Neural Correlates of Working Memory}

The prefrontal cortex (PFC) is the central neuronal component implementing WM. This was discovered in early lesion studies (Pribram et al., 1952) as well as in electrophysiological recordings in monkeys (Kubota and Niki, 1971; Fuster and Alexander, 1971). However, there are diverse results regarding the type of activity observed in the PFC during WM operation. On the one hand, it was reported that during performing a WM task, individual neurons in the PFC show persistent activity during the delay phase (Fuster and Alexander, 1971; Funahashi et al., 1989). The delay phase denotes the time interval in between the presentation of a stimulus and the recall of the information about this stimulus to perform a certain task. The level of persistent activity in PFC during the delay phase has been shown to predict the performance of subjects in the respective WM task (Constantinidis et al., 2001; Zhou et al., 2013; Wimmer et al., 2014). Furthermore, different neurons in the PFC have been show to stably code for different features of the stimulus during the delay phase (Goldman-Rakic, 1995; Constantinidis and Wang, 2004). This suggests that information stored in WM is represented by persistent activity of specific neurons in the PFC. On the other hand, other studies observe a much larger heterogeneity of the activity traces of neurons in the monkey PFC during the delay phase of WM tasks (Jun et al., 2010; Hussar and Pasternak, 2012). The representation of the maintained information is found to depend on the time since the presentation of the stimulus (Hussar and Pasternak, 2012). Thus, stored information is not represented by a single stable pattern of activity but rather by a transient sequence, or trajectory, of different activity states. This kind of transient information coding has also been identified in the PFC of rats which perform a WM task (Zhang et al., 2015). Independent of WM, neuronal information representation in the form of transient activity has also been found in other organisms and brain areas (Mazor and Laurent, 2005).

In summary, experimental results on the neural correlates of WM are diverse with some experiments indicating that WM relies on storing information by persistent activity while other experiments find that information is rather stored in complex transient trajectories of different neuronal patterns.

\subsubsection{Neurocomputational Models of Working Memory}

The different experimental findings regarding the representation of information stored in WM give rise to an ongoing debate about the kind of dynamics underlying the neuronal systems implementing WM. (D'Esposito, 2007; Mongillo et al., 2008; Barak and Tsodyks, 2014; Trübutschek et al., 2017). As a first option, WM functionality 
might rely on attractor-dominated neuronal dynamics. As a second option, WM might exploit the properties of purely transient neuronal dynamics to store, retrieve and process the stream of incoming stimuli. Both notions have lead to different neurocomputational models of WM. Additionally, there are also other approaches which propose that information in WM is encoded in the different properties of rhythmic activity (Siegel et al., 2009; Salazar et al., 2012; Buschman et al., 2012) or by fast and short-lasting changes of synaptic weights (Sandberg et al., 2003; Mongillo et al., 2008; Sugase-Miyamoto et al., 2008; Eriksson et al., 2015).

The idea that WM might operate based on attractor dynamics basically dates back to Hebb's second postulate (Section 2.2) which states that WM relies on reverberating activity in CAs. This is compatible with the electrophysiological experiments which find persistent activity during the delay period of typical WM tasks (Section 2.4.2). A variety of different mechanisms have been proposed to underly this persistent activity (Durstewitz et al., 2000). In ring models, for instance, neurons representing different orientations or locations of a stimulus interact via mutually inhibition and selfrecurrence. The occurrence of a stimulus results in persistent activity in the neurons which represents the to be remembered orientation (Compte et al., 2000). Once the stimulus vanishes, this bump of activity slowly drifts away from the original position resulting in a time-dependent loss of precision as also observed in experiments. Predictions from this model could actually be confirmed in activities recorded in monkey PFC (Wimmer et al., 2014). Slow divergence of the activity along a continuum of attractor states has been proposed to explain different tuning properties of cells in different phases of a WM task (Drover, 2014). Attractor dynamics, which reproduce the frequently observed persistent activity during the delay period, has also been modeled in a very detailed neurobiological model involving several populations of spiking neuronal networks and different kinds of synaptic interactions (Deco and Rolls, 2003).

The second class of proposed WM mechanisms is based on dynamic patterns and transient dynamics (Stokes et al., 2013). In these models, every incoming stimulus drives the neuronal system along a characteristic trajectory. This results in different activities of individual neurons at different point of times during the delay period of a WM task and might explain the corresponding experimental findings (Section 2.4.2). Transient dynamics possess a larger computational power than attractordominated dynamics (Rabinovich et al., 2008a) and offer a powerful mechanism to combine the internal neuronal dynamics with the signals arriving due to external stimuli (Buonomano and Maass, 2009). Additionally, they have the potential to allow multiple computations to be performed simultaneously (Maass, 2011; Tetzlaff et al., 2015). Accordingly, models relying on different types of transient activities have been proposed as alternative models of working memory operation (Barak and Tsodyks, 2014). One of these types are reservoir networks which are discussed in more detail in the next section.

\subsubsection{Reservoir Networks}

The paradigm of reservoir computing has been simultaneously and independently emerged under the name of echo state networks (ESNs) in the field of machine learning (Jaeger, 2001) and as liquid state machines (LSMs) in the field of computational neuroscience (Maass et al., 2002). Accordingly, ESNs are implemented in networks of abstract rate-coded neurons while the LSMs employ networks of complex spiking neurons. The unifying idea of both approaches is based on the same observation: In a recurrent neuronal network with certain generic properties, it is not necessary to 
adapt the strength of the recurrent synapses in order to obtain a system that transforms possibly time-dependent input traces into some desired output signals. Instead, it suffices to train a memoryless linear readout (Lukoševičius et al., 2012). Loosely speaking, all kinds of different nonlinear transformations are performed automatically within the recurrent neuronal network. For the readout, it therefore suffices to collect and superimpose these transformations in an optimal way. Thus, even the training of complex nonlinear computations can be reduced to a linear optimization problem. Network architectures which are trained in this manner, are called reservoir networks (Lukoševičius et al., 2012). A reservoir network consists of a large recurrent neuronal network called the generator network and a set of readout units.

In the most basic reservoir network architecture, there is no feedback from readout neurons back to the generator network. Introducing such feedback can turn the reservoir into a universal function approximator that is able to carry out all possible kinds of computation on the incoming time-dependent stimuli (Maass et al., 2007). A reservoir training method that is especially tailored for architectures with feedback signals from the readout units is called first-order reduced and controlled error (FORCE) learning (Sussillo and Abbott, 2009). The key idea of FORCE learning is to adapt the readout weights fast enough to make sure that already during the training session, the feedback from the readout units resembles the actual feedback during exploitation of the reservoir network. When training the reservoir network using the method originally proposed for ESNs, in contrast, the feedback signals have to be artificially set to the respective target signals during the training of the network (Jaeger, 2001). This may result in diverging network dynamics once this feedback clamping is released.

Reservoir networks have been discussed as possible models of WM (Barak et al., 2010; Sussillo et al., 2015; Barak and Tsodyks, 2014). In addition to using the plain reservoir network architecture without feedback from the readout units, also the introduction of specific readout units which establish feedback loops to store relevant information has been proposed (Maass et al., 2007; Jaeger and Eck, 2008; Pascanu and Jaeger, 2011). In Chapter 3, we study both of these architecture variants.

\subsection{Memory of Rhythmic Neuronal Signals}

While LTM and WM constitute very explicit forms of memory, there are many processes in the brain which can be interpreted as a kind of more implicit memory. For instance, such a process may be given by the ongoing generation of a rhythmic signal at a specific memorized frequency. This memorized frequency might have been extracted, for example, from a short transient control signal generated by a higher-level neuronal system. In general, rhythmic signals are of central importance in many areas of biological life. Well known examples of rhythmic processes include the cardiac rhythm, circadian rhythms and in particular all kinds of locomotion (Winfree, 1967; Barkai and Leibler, 2000; Goldbeter et al., 2012). Many of these rhythmic processes in biological creatures are controlled by so called central pattern generators (CPGs) (Hooper, 2001; Marder and Bucher, 2001). CPGs are specific neuronal circuits which are able to produce periodic activity signals without receiving any periodic input signal. 


\subsubsection{Central Pattern Generators}

Several pieces of evidence for the existence of CPGs in both mammalian and nonmammalian animals stem from fictive locomotion experiments (Wilson, 1961; Delcomyn, 1980; Wallén and Williams, 1984; Clarac and Pearlstein, 2007; Guertin, 2009). During these experiments, all relevant sensory inputs are surgically removed from the animal. In this state, activity in the neuronal areas responsible for motor control is evoked, for instance, by the addition of certain neuromodulators. Apart from a different frequency, the hereby recorded patterns of neuronal activity resemble strongly the ones recorded during normal locomotion of the intact animal. Thus, the nervous system is able to produce these patterns without having to rely on sensory feedback.

Studies of theoretical models of CPGs have been performed on many different levels of abstractions. On the one hand, simulations of detailed biophysical models of networks of spiking neurons have been shown to be able to produce rhythmic signals in the biologically relevant frequency range (e.g. Hellgren and Grillner, 1992; Tråvén et al., 1993). On the other hand, abstract mathematical models of oscillatory systems reducing the level of complexity to a minimum have been used to derive general principles and properties of such self-sustained oscillations (e.g. Matsuoka, 1985; Landsman and Slotine, 2012).

Based on the widespread and successful occurrence of CPGs in biological organisms, models of CPGs have been employed in a number of robotic control applications (Ijspeert, 2008). This includes the control of biped robots (Nakamura et al., 2007; Nassour et al., 2014; Santos et al., 2017), quadruped robts (Righetti and Ijspeert, 2008), and hexapod robots (Steingrube et al., 2010; Pinto et al., 2012) as well as the optimization of the control of swimming and crawling robots (Lu et al., 2005; Inoue et al., 2007; Crespi and Ijspeert, 2008). In contrast to locomotion schemes relying on purely reflex control mechanisms (Foth and Bässler, 1985; Cruse et al., 1995), locomotion controlled by CPG models has been show to be more stable and robust (Kimura et al., 2001; Righetti and Ijspeert, 2008).

Most CPG models produce output signals at a single predefined frequency. In order to obtain a CPG which is able to adapt its output frequency to the frequency of a short-lasting transient input signal as described above, additional mechanisms are required. One such mechanism has been described by Righetti et al., (2006) under the name of adaptive frequency oscillator (AFO).

\subsubsection{Adaptive Frequency Oscillators}

Righetti et al., (2006) developed a universal mechanism which is able to adapt the intrinsic frequency of a nonlinear oscillator based on the frequency of an external periodic signal coupled to the oscillator. This method is applicable to a variety of different mathematical models of oscillators including harmonic oscillators (Andronov et al., 1971) and highly non-harmonic oscillators (Rayleigh, 1877; Van der Pol, 1920). Note that the mechanism modifies the intrinsic frequency of the respective oscillators permanently. This is in contrast to the phenomenon of entrainment which describes the just transient frequency locking of an oscillator to an external perturbation (Buchli et al., 2006b). Oscillator models which are able to adapt their intrinsic frequency based on the mechanism proposed by Righetti et al., (2006) are referred to as adaptive frequency oscillators (AFOs).

AFOs have been successfully employed in a number of robotic applications such as pendulum swing-up problems (Spong, 1995; Furuta, 2003), assistance of human 
locomotion (Ronsse et al., 2011; Tropea et al., 2015), frequency analysis of an input signal (Buchli et al., 2008), and the construction of limit cycle systems of arbitrary shape (Righetti et al., 2009). In particular, they have been used as models of CPGs which are able to autonomously adapt their frequency to beneficial frequencies of the system under control, for instance, in quadruped robots (Buchli et al., 2006a), biped robots (Santos et al., 2017) and swimming robots (Wang et al., 2013). There is, however, a common pitfall of the AFO mechanism which all of these applications suffer off: In order to achieve precise adaptation, the learning rates of the mechanism has to be chosen small which results in long adaptation times. In Chapter 5, this trade-off is investigated in more detail. 



\section{Chapter 3}

\section{Neuronal Dynamics of Working Memory}

Working memory (WM) is characterized by its ability to both store information about past stimuli on short timescales as well as to process and manipulate the stored information (Baddeley and Hitch, 1974). The type of neuronal network dynamics underlying these two abilities, to store and to process information, is a matter of ongoing debate. The dynamics might either be dominated by an interaction of different attractor states or, instead, follow attractor-less transient dynamics. In theories which follow the first assumption, the attractor states in the WM system are commonly believed to be represented by persistent neuronal activity (Durstewitz et al., 2000). According to the second assumption, WM is considered to be realized by a complex and non-stationary flow of neuronal activity within a possibly large neuronal population (Stokes et al., 2013). Interestingly, experimental results as well as theoretical considerations yield support for both of these seemingly contradictory assumptions.

Considering the assumption that WM is implemented by a non-stationary flow of neuronal activity, reservoir networks (Jaeger, 2001; Maass et al., 2002) are the most prominent and intensively studied class of neuronal network models which operate on transient neuronal dynamics. Indeed, they have been shown (Jaeger, 2002; Bertschinger and Natschläger, 2004; Lukoševičius and Jaeger, 2009; Schrauwen et al., 2009) to possess both high computational power (processing of information) as well as the ability to transiently represent recent stimuli on short timescales (storing of information). As a consequence, different studies (e.g. Barak et al., 2013; Dasgupta et al., 2013; Barak and Tsodyks, 2014; Cheng et al., 2015) have presented reservoir networks which are able to perform typical WM tasks. Most of these studies, however, neglect an important requirement for robust WM operation: When interacting with the environment, neither humans nor animals can rely on precisely and predictably timed input stimuli. In contrast, stimuli commonly arrive in a highly temporally unpredictable manner. Interestingly, as discussed in Section 2.4.1, the performance of human subjects performing an established WM task, the n-back task, is not altered by presenting the individual stimuli with unpredictable timing (Koppe et al., 2014). Plausible neuronal models of WM have to be able to provide the same level of tolerance with respect to unreliably timed input stimuli. Whether reservoir networks are able to provide this level of robustness has not yet been studied.

According to the first sub-hypothesis of this thesis (compare Figure 1.1), WM operation depends on both transient dynamics and attractor states to enable robust information storage and complex computations. After introducing the methods and theoretical foundations of this chapter (Section 3.1), we verify this sub-hypothesis (Section 3.2). First, we show that reservoir networks operating with purely transient dynamics are able to solve the n-back task with predictable stimulus timing 
(Section 3.2.1) but not the n-back task with unpredictable stimulus timing (Section 3.2.2). Second, we demonstrate that the introduction of feedback loops via additional readout units, which represent the relevant information for the n-back task, restores the performance of reservoir network in the n-back task with unpredictable stimulus timing (Section 3.2.3). We show that this robustness of the performance is caused by introducing structure into the dynamics of the system in the form of distinct attractor states (Section 3.2.4). Nevertheless, we demonstrate that only sufficiently complex transient dynamics enables the precise production of the target signal in the n-back task (Section 3.2.5). Based on this analysis, we propose a functional separation between attractor states and transient dynamics in the neuronal systems implementing WM: Attractor states are responsible for robust information storage and transient neuronal dynamics enable complex computations.

The line of argument and the corresponding conclusions in this chapter have been published in the following journal article:

T. Nachstedt and C. Tetzlaff (2017). Working Memory Requires a Combination of Transient and Attractor-Dominated Dynamics to Process Unreliably Timed Inputs. Scientific Reports 7, p. 2473. DOI: 10.1038/s41598-017-02471-z.

\subsection{Methods}

In this section, first, we give a short summary of the theory of the type of recurrent networks employed as reservoir networks in this study. Next, we present the actually used reservoir network architecture, the two used methods to train these reservoir networks and the measure for performance evaluation. Afterwards, we define the formulation of the n-back task for the reservoir networks. Finally, we summarize the method of dimensionality reduction via principal component analysis.

\subsubsection{Recurrent Networks at the Edge of Chaos}

Following the approach of Sussillo and Abbott, (2009), the reservoir networks used in this study are given by random networks of $N$ coupled nonlinear neurons as originally studied by Sompolinsky et al., (1988). We describe each neuron by a time-dependent membrane potential $u_{i}(t)$ with $i \in\{1, \ldots, N\}$. The membrane potential determines the firing rate $F_{i}(t)$ of the neuron via a nonlinear firing rate function $\phi$ such that $F_{i}(t)=\phi\left(u_{i}(t)\right)$. The firing rate function is given by the tangent hyperbolic: $\phi(x)=\tanh (x)$. The dynamics of every neuron is described by a firstorder differential equation:

$$
\tau \dot{u}_{i}=-u_{i}+\sum_{j=1}^{N} w_{i j} F_{j}=-u_{i}+\sum_{j=1}^{N} w_{i j} \phi\left(u_{j}\right)
$$

with a time constant $\tau$. Importantly, we assume the weight $w_{i j}$ of the synapse from neuron $j$ to neuron $i$ to be an independent random variable drawn from a Gaussian distribution with zero mean and variance $\sigma_{w}^{2}=g^{2} / N$ with a gain parameter $g>0$. As analytically shown by Sompolinsky et al., (1988), in the limit $N \rightarrow \infty$, the long-term dynamics of this network depends critically on the parameter $g$.

In the case of $g<1$, the system possesses a single stable equilibrium at the origin, i.e. $u_{i}=0$ for all $i$. This is related to the circular law for random matrices which states that for $N \rightarrow \infty$, the spectral radius of the matrix $W=\left(w_{i j}\right)$ converges to $g$ (Figure $3.1 \mathrm{a}$; Tao et al., 2010). Thus, the real parts $\operatorname{Re}\left(\lambda_{i}\right)$ of all eigenvalues $\lambda_{i}$ 


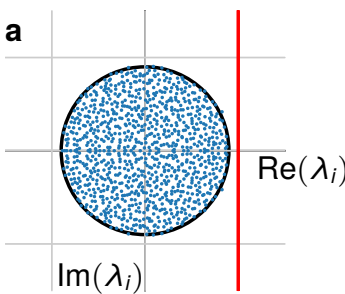

d

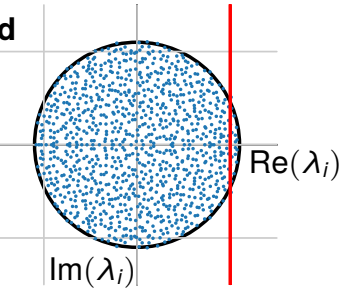

g

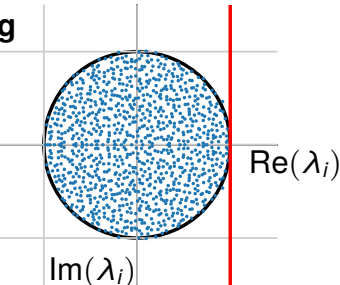

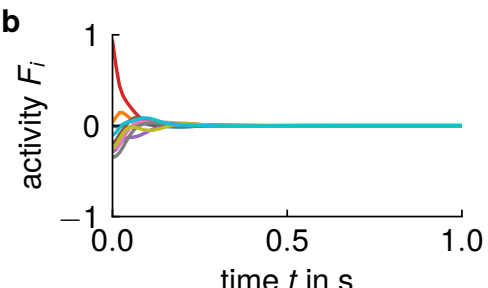

$g=1.1:$ chaotic dynamics

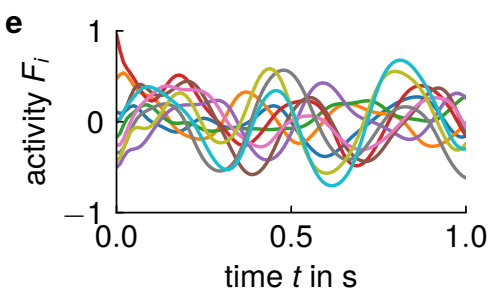

$g=1.0:$ at the edge of chaos

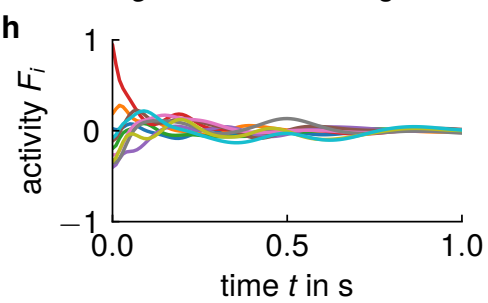

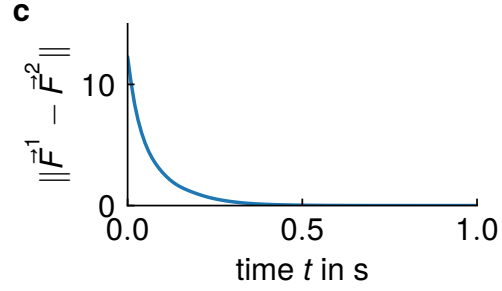

f
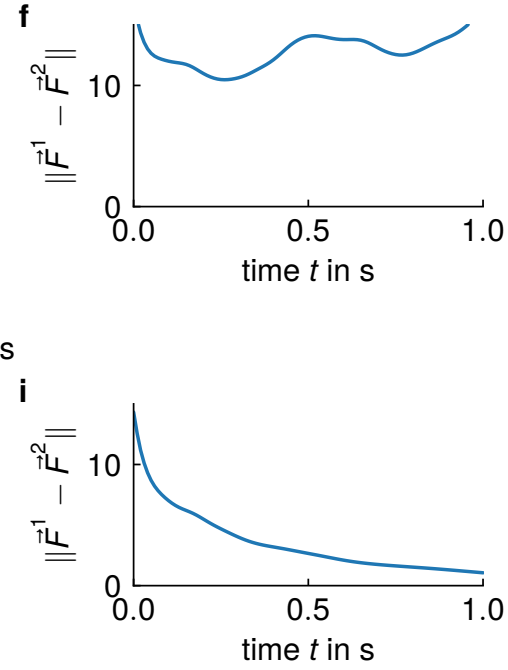

Figure 3.1: Dynamics at the edge of chaos in random neuronal networks. The dynamics of random recurrent networks of $N$ neurons with a firing rate function $\phi(x)=\tanh (x)$ and a weight matrix $W=\left(w_{i j}\right)$ drawn from a normal distribution with zero mean and variance $g^{2} / N$ depends on the value of $g$. Here, the network contains $N=1000$ neurons and has a time constant $\tau=10 \mathrm{~ms}$. (a, b, c) For $g<1$, the network exposes stable dynamics with a single stable equilibrium at zero activity. (d, e, f) For $g>1$, chaotic dynamics emerge. (g, $\mathbf{h}, \mathbf{i})$ The critical value $g=1$ is called the edge of chaos. To ensure the network is still slightly below the edge of chaos, we make sure that the real part of all eigenvalues $\lambda_{i}$ of $W$ are smaller than $1: \max _{i} \operatorname{Re}\left(\lambda_{i}\right)<1$. Networks operating slightly below the edge of chaos have been shown to offer optimal computational power (Bertschinger and Natschläger, 2004; Legenstein and Maass, 2007). (a, $\mathbf{d}, \mathbf{g})$ The spectrum of eigenvalues $\lambda_{i}$ of the weight matrix $W$ in the complex plane and the resulting spectral radius is directly related to the gain parameter $g$ by the circular law for random matrices. The individual eigenvalues $\lambda_{i}$ of the random matrix are depicted in blue. The circle with radius $g$ in the complex plane is shown in black. The red line indicates the threshold for chaotic behavior at $\operatorname{Re}\left(\lambda_{i}\right)=1$. (b, e, $\left.\mathbf{h}\right)$ We project an external perturbation of unit amplitude via random weights onto half of the network neurons for $-1 \mathrm{~s}<t<0 \mathrm{~s}$. Shown are the resulting dynamics of the activities $F_{i}$ of ten randomly chosen neurons in the network. Observe the different time scales of the decay to zero for $g=0.9$ and $g=1.0$. (c, $\mathbf{i}, \mathbf{f})$ We project a second external perturbation of unit amplitude via random weights onto the second half of network neurons as compared to $b$, $e$ and $h$. The norm of the difference between the vector $\vec{F}^{1}$ of all neuron activities observed in (b), (e) and (h) and the vector $\vec{F}^{2}$ of the neuron activities observed here characterizes the decay of the information about the stimulus type over time. 
of $W$ are smaller than 1: $\max _{i} \operatorname{Re}\left(\lambda_{i}\right)<1$. Due to the leak term of the membrane potential (Equation 3.1), this means that the real parts of all eigenvalues of the Jacobian matrix of the network dynamics evaluated at the zero activity equilibrium are negative. Accordingly, the zero activity equilibrium is stable (Sompolinsky et al., 1988). Thus, if the network state is pushed away from this equilibrium state by an external perturbation, the network dynamics drives the system back to the equilibrium state (Figure $3.1 \mathrm{~b}$ ). As a result, the difference between the network states resulting from two different perturbations eventually decays to zero (Figure 3.1 c).

In the case of $g>1$, in contrast, no stable solutions exist and the system exposes a dynamics known as deterministic chaos. Now, the spectral radius of the weight matrix $W$ is larger than 1 . Accordingly, also the maximum real part $\max _{i} \operatorname{Re}\left(\lambda_{i}\right)$ of the eigenvalues $\lambda_{i}$ of $W$ is larger than 1 (Figure $3.1 \mathrm{~d}$ ). As a consequence, the Jacobian matrix of the network dynamics evaluated at the equilibrium possesses eigenvalues with positive real parts. Thus, a network which originally resides in the equilibrium at the origin of the activity space and receives an external perturbation does not return to this equilibrium. Instead, it exposes ongoing and not stereotyped activity (Figure $3.1 \mathrm{e}$ ). In particular, the difference of the network states resulting from two different perturbations does not decay but remains large (Figure 3.1 f).

Within the context of reservoir computing, the dynamics near the boundary between ordered dynamics $(g<1)$ and chaotic dynamics $(g>1)$, that is for $g=1$, is of special interest (Figure $3.1 \mathrm{~g}$ ). Networks operating slightly below this boundary, i.e., whose largest real part $\max _{i} \operatorname{Re}\left(\lambda_{i}\right)$ of all eigenvalues of $W$ is still below 1 , are characterized by a slow decay of activity (Figure $3.1 \mathrm{~h}$ ). This intuitively corresponds to a high memory capacity and is accompanied by a slow decay of the difference of the network states resulting from different external perturbations (Figure 3.1 i). Based on different theoretical studies, networks operating slightly below this so-called edge of chaos are assumed to offer optimal computational power (Bertschinger and Natschläger, 2004; Legenstein and Maass, 2007). The reservoir networks trained in the following operate in this regime, i.e., slightly below the edge of chaos.

\subsubsection{Reservoir Network Training Algorithms}

As described in Section 2.4.4, different methods to optimize the readout weights of a reservoir network have been developed. Within this thesis, the echo state network procedure (Jaeger, 2001) (in the following "ESN learning") and the FORCE learning algorithm (Sussillo and Abbott, 2009) are employed. While the former is an offline method especially suitable for networks without feedback from the readout units, the latter adapts the weights on-line during operation of the network and is tailored for networks relying on feedback signals. Before introducing these two methods, we describe the used network architecture.

\section{Network Architecture}

Following the terminology of Sussillo and Abbott, (2009), we divide the reservoir network into the input units, the generator network and the readout units. Every neuron $i$ of the $N_{\mathrm{G}}$ neurons within the generator network receives signals from exactly one randomly chosen input signal $k$ via a synapse with a weight $w_{i k}^{\mathrm{GI}}$ drawn from a normal distribution with zero mean and variance $g_{G \mathrm{GI}}^{2}$. The generator neurons themselves are fully recurrently connected. The weight $w_{i j}^{\mathrm{GG}}$ of the synapse transmitting signals from neuron $j$ to neuron $i$ within the generator network is drawn from a normal distribution with zero mean and standard deviation $g_{\mathrm{GG}} / \sqrt{N_{\mathrm{G}}}$ (Figure 3.2, right). Note that 
the parameter $g_{\mathrm{GG}}$ corresponds to the gain parameter $g$ in Section 3.1.1. Thus, the value of $g_{\mathrm{GG}}$ determines the type of dynamics within the generator network. In the following, we usually choose $g_{\mathrm{GG}}=1$ but only use weight matrices $W^{\mathrm{GG}}=\left(w_{i j}^{\mathrm{GG}}\right)$ for which the maximum real part of an eigenvalue $\lambda_{i}$ is smaller than one: $\max _{i} \operatorname{Re} \lambda_{i}<1$. Additionally, the neurons in the generator network may receive feedback from the $N_{\mathrm{R}}$ readout units. The weight $w_{i l}^{\mathrm{GR}}$ of a synapse transmitting signals from readout unit $l$ to neuron $i$ within the generator network is drawn from a normal distribution with zero mean and variance $g_{\mathrm{GR}}^{2} / \sqrt{N_{R}}$. Thus, a value of $g_{\mathrm{GR}}=0$ corresponds to disabled feedback signals.

The dynamics of neuron $i, i \in\left\{1, \ldots, N_{G}\right\}$, within the generator network is determined by a leaky membrane potential $u_{i}^{\mathrm{G}}$ described by

$$
\tau \frac{\mathrm{d} u_{i}^{\mathrm{G}}}{\mathrm{d} t}=-u_{i}^{\mathrm{G}}+\sum_{j=1}^{N_{\mathrm{G}}} w_{i j}^{\mathrm{GG}} F_{j}^{\mathrm{G}}+\sum_{k=1}^{N_{\mathrm{I}}} w_{i k}^{\mathrm{GI}} I_{k}+\sum_{l=1}^{N_{\mathrm{R}}} w_{i l}^{\mathrm{GR}} R_{l} .
$$

Here, the time constant $\tau=10 \mathrm{~ms}$ determines the timescale of the dynamics and $N_{\mathrm{G}}$, $N_{\mathrm{I}}$, and $N_{\mathrm{R}}$ give the number of neurons within the generator network, the number of input signals and the number of readout units, respectively. The firing rate $F_{i}^{\mathrm{G}}$ of neuron $i$ within the generator network is given by the hyperbolic tangent of its membrane potential:

$$
F_{i}^{\mathrm{G}}=\phi\left(u_{i}^{\mathrm{G}}\right)=\tanh \left(u_{i}^{\mathrm{G}}\right) .
$$

The input signals $I_{k}$ are externally controlled and vary between 0 and 1 . The activity of the linear readout unit $l, l \in\left\{1, \ldots, N_{\mathrm{R}}\right\}$, is given by

$$
R_{l}=\sum_{i=1}^{N_{\mathrm{G}}} w_{l i}^{\mathrm{RG}} F_{i}^{\mathrm{G}} .
$$

Depending on the used reservoir network training algorithm, it may be necessary to define initial values for the readout weights $w_{l i}^{\mathrm{RG}}$. In these cases, the initial values are drawn from a normal distribution with zero mean and standard deviation $1 / \sqrt{N_{G}}$.

We numerically integrate the network dynamics by using the Euler method (Butcher, 2003) with a time step of $\Delta t_{\text {euler }}=1 \mathrm{~ms}$.

\section{Echo State Network Learning}

The echo state network (ESN) learning procedure as proposed by Jaeger, (2001) relies on sampling the network activity while driving the network with a sample of the input signal. Hereby, the first $s_{\text {discard }}$ time steps after the onset of the input signal are discarded to avoid transient effects of the network's initial conditions. At every one of the following $s_{\text {train }}$ time steps, the activity $F_{i}^{\mathrm{G}}\left(t_{s}^{\text {train }}\right)$ of all reservoir neurons $i \in\left\{1, \ldots, N_{\mathrm{G}}\right\}$ is collected into a row of the state matrix $M$ such that the final dimension of $M$ is $s_{\text {train }} \times N_{G}$. Here, $t_{s}^{\text {train }}$ denotes the actual time at which state number $s$ is collected. In parallel, the corresponding target signals $f_{l}\left(t_{s}^{\text {train }}\right)$, $l \in\left\{1, \ldots, N_{R}\right\}$, for the readout units are collected into columns of a teacher matrix $T$ of dimension $s_{\text {train }} \times N_{R}$. Note that in the case of non-zero feedback connections from the readout units back to the neurons within the generator network $\left(g_{\mathrm{GR}}>0\right)$, the ESN procedure uses so-called feedback-clamping during the training process: The signal transmitted from readout unit $R_{l}, l \in\left\{1, \ldots, N_{R}\right)$, to the generator network is artificially set to the respective target value $f_{l}\left(t_{s}^{\text {train }}\right)$ modified by Gaussian noise with a variance $\sigma_{\text {noise }}^{2}$. Concerning the strength of the applied noise, no clear guideline 
how to choose its amplitude is available. Throughout this thesis, we use a value of $\sigma_{\text {noise }}=0.1$.

After the sampling phase, the readout weights are determined by minimizing the mean squared error (MSE) of the resulting overdetermined system of equations with the help of the Moore-Penrose pseudoinverse $M^{+}$of the state matrix $M$ :

$$
W^{\mathrm{RG}}=\left(M^{+} T\right)^{T} .
$$

The root mean squared error RMSE $E_{\text {train }}$ of the readout signals, as they would have been read out during training by the optimized weight matrix $W^{\mathrm{RG}}$, provides information on how well the reservoir is able to approximate the trained sample of the target signal. This value is normalized by the root mean square $\mathrm{RMS}_{\text {target }}$ of the recorded target signal to obtain the training error $E_{\text {train: }}$ :

$$
\begin{aligned}
E_{\text {train }}=\frac{\mathrm{RMSE}_{\text {train }}}{\mathrm{RMS}_{\text {target }}} & =\frac{\sqrt{\sum_{s=1}^{s_{\text {train }}} \sum_{l=1}^{N^{\mathrm{R}}}\left(f_{l}\left(t_{s}^{\text {train }}\right)-\sum_{i=1}^{N^{\mathrm{G}}} w_{l i}^{\mathrm{RG}} F_{i}^{\mathrm{G}}\left(t_{s}^{\text {train }}\right)\right)^{2}}}{\sqrt{\sum_{s=1}^{s_{\text {train }} \sum_{l=1}^{N^{\mathrm{R}}}\left(f_{l}\left(t_{s}^{\text {train }}\right)\right)^{2}}}} \\
& =\sqrt{\frac{\left\|M \cdot\left(W^{\mathrm{RG}}\right)^{T}-T\right\|_{\mathrm{F}}}{\|T\|_{\mathrm{F}}}}
\end{aligned}
$$

where $\|\cdot\|_{F}$ denotes the Frobenius norm.

\section{FORCE Learning}

In contrast to the ESN learning procedure, first-order reduced and controlled error learning (FORCE, Sussillo and Abbott, 2009) relies on an on-line adaptation of the network where the weight matrix $W^{\mathrm{RG}}$ is modified in every simulation step. The idea of FORCE learning is to allow very rapid weight modifications. As a result, the signal at the readout units is close to the target signal right from the very beginning. This removes the necessity of feedback-clamping as used in the ESN learning approach.

As in the ESN approach, the network is first driven by the input signal for $s_{\text {discard }}$ time steps to eliminate the influence of the initial conditions. Afterwards, at every time step $t_{s}$ with $s \in\left\{1, \ldots, s_{\text {train }}\right\}$, the recursive least-squares (RLS) algorithm (Haykin, $2002)$ is utilized to adapt the readout weight vectors $\vec{w}_{l}^{\mathrm{RG}}=\left(w_{l, 1}^{\mathrm{RG}}, \ldots, w_{l, N_{\mathrm{G}}}^{\mathrm{RG}}\right)^{T}, l \in$ $\left\{1, \ldots, N_{R}\right\}$, according to

$$
\vec{w}_{l}^{\mathrm{RG}}\left(t_{s}\right)=\vec{w}_{l}^{\mathrm{RG}}\left(t_{s-1}\right)-e_{l}^{-}\left(t_{s}\right)\left(P\left(t_{s}\right) \vec{F}^{\mathrm{G}}\left(t_{s}\right)\right)^{T} .
$$

Here, $\vec{F}^{\mathrm{G}}\left(t_{s}\right)=\left(F_{1}^{\mathrm{G}}\left(t_{s}\right), \ldots, F_{N_{\mathrm{G}}}^{\mathrm{G}}\left(t_{s}\right)\right)^{T}$ is the activity vector of the neurons within the generator network and $e_{l}^{-}\left(t_{s}\right)$ denotes the difference between the readout signal with index $l$ and the corresponding target signal $f_{l}\left(t_{s}\right)$ prior to the weight update:

$$
e_{l}^{-}\left(t_{s}\right)=\left(\vec{w}_{l}^{\mathrm{RG}}\left(t_{s-1}\right)\right)^{T} \vec{F}_{\mathrm{G}}\left(t_{s}\right)-f_{l}\left(t_{s}\right) .
$$

Note that already the updated weights $\vec{w}_{l}^{\mathrm{RG}}\left(t_{s}\right)$ are used to determine the actual readout signals $R_{l}\left(t_{s}\right)$ in the given time step. The square matrix $P\left(t_{s}\right)$ of shape $N_{\mathrm{G}} \times N_{\mathrm{G}}$ has an initial value of $P\left(t_{0}\right)=\alpha^{-1} \mathbb{1}$. Here, $\mathbb{1}$ denotes the identity matrix. 
$P\left(t_{s}\right)$ is updated in every time step according to

$$
P\left(t_{s}\right)=P\left(t_{s-1}\right)-\frac{P\left(t_{s-1}\right) \vec{F}^{\mathrm{G}}\left(t_{s}\right)\left(\vec{F}^{\mathrm{G}}\left(t_{s}\right)\right)^{T} P\left(t_{s-1}\right)}{1+\left(\vec{F}^{\mathrm{G}}\left(t_{s}\right)\right)^{T} P\left(t_{s-1}\right) \vec{F}^{\mathrm{G}}\left(t_{s}\right)} .
$$

The value of $\alpha$ has to be adjusted according to the specific optimization problem at hand. Lower values of $\alpha$ allow for faster weight adaptations in the early learning phase. In the presence of feedback connections from the readout units back to the neurons within the generator network, however, this may reduce stability of the FORCE method (Sussillo and Abbott, 2009).

\section{Performance Evaluation}

We evaluate the actual performance of the networks trained by either of the two training methods on test data that was not used during training. For this, we iterate the network for further $s_{\text {test }}$ simulation steps and compare the readout signal $R_{l}\left(t_{s}^{\text {test }}\right)$ at every sampled time $t_{s}^{\text {test }}, s \in\left\{1, \ldots, s_{\text {test }}\right\}$, with the respective target signal $f_{l}\left(t_{s}^{\text {test }}\right)$ to obtain the error $E$ :

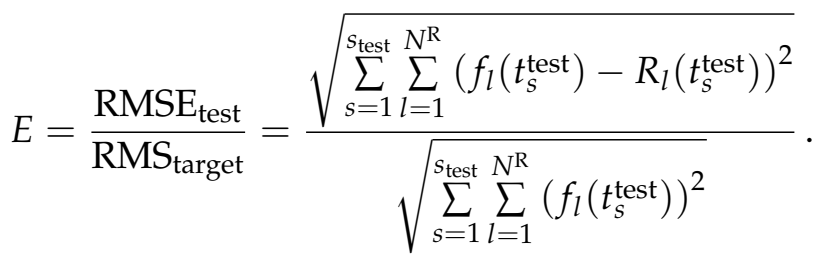

\subsubsection{An N-Back Task for Reservoir Networks}

In order to evaluate whether reservoir networks may solve the n-back task, we have to translate the experimental paradigm of the n-back task into a set of input signals which represent the different stimuli in this task. Additionally, we have to define the shape of the target signal. In the following, if not stated otherwise, we always choose $n=2$. Thus, the network has to compare the current incoming stimulus with the second last stimulus it received and to produce a corresponding output signal.

\section{Input Signal}

The network receives pulse-shaped input signals from $N_{I}=2$ different input pathways labeled A and B (Figure 3.2, top left). Every occurrence of a pulse at either of the two input pathways represents the presentation of a respective stimulus. The shape of the pulses is given by a convolution of a constant signal with unit magnitude and length $t_{\text {pulse }}=25 \mathrm{~ms}$ and a normalized Gaussian window with standard deviation $\sigma_{\text {smooth }}=5 \mathrm{~ms}$. The individual pulses are separated by interstimulus intervals $\Delta t_{i}$ which are drawn from a normal distribution with mean $\mu_{\Delta t}$ and standard deviation $\sigma_{\Delta t}$. To avoid overlaps between the different input stimuli, we additionally

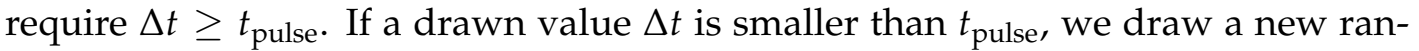
dom value. The pulse signals at the two input pathways are superposed by a small noisy background signal given by a white noise process with a standard deviation of $\sigma_{\mathrm{WN}}=0.001$. 


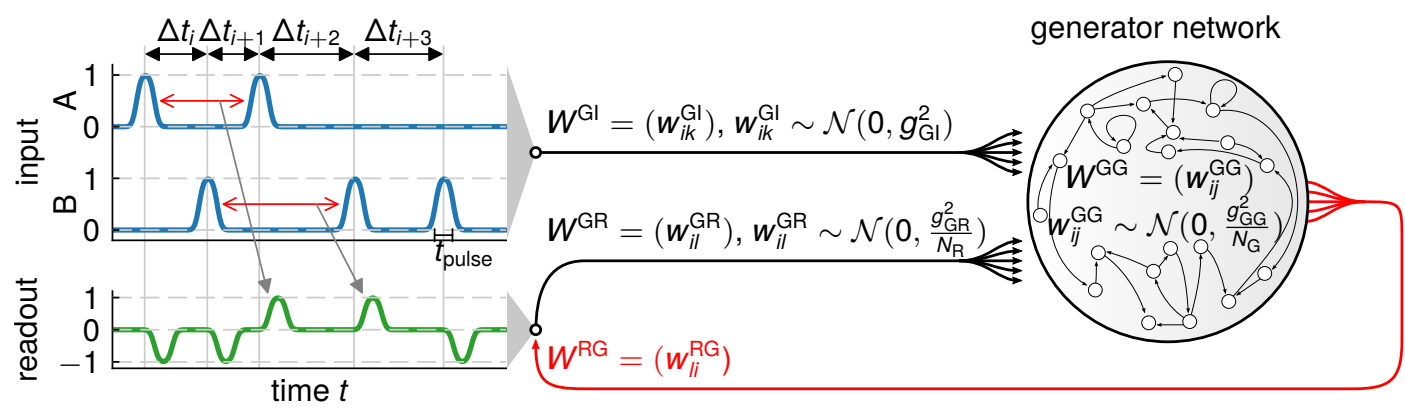

Figure 3.2: Setup for testing a reservoir's performance in the $\mathbf{n}$-back task (here $n=2$ ). The input signal consists of two channels representing the two different stimuli, namely $A$ and $B$. Every stimulus has an equal probability of being either of the A- or the B-type and is represented by a smooth pulse of duration $t_{\text {pulse. }}$. The individual stimuli are separated by time intervals $\Delta t_{i}$ drawn from a normal distribution $\mathscr{N}$ with mean $\mu_{\Delta t}$ and variance $\sigma_{\Delta t}^{2}$. The inputs are projected into the generator network by a synaptic weight matrix $W^{\mathrm{Gl}}$ whose elements $w_{i k}^{\mathrm{Gl}}$ are drawn from $\mathscr{N}\left(0, g_{\mathrm{Gl}}^{2}\right)$. Similar to a regular $\mathrm{n}$-back task, the network has to produce output pulses of a defined shape whenever the type of the current stimulus corresponds to the type of the stimulus two steps before (compare arrows). During learning, the readout weight matrix $W^{R G}$ is adapted (red). The readout signal is fed back into the generator network via a synaptic weight matrix $W^{\mathrm{GR}}$ with elements $w_{i l}^{\mathrm{GR}}$ drawn from $\mathscr{N}\left(0, g_{\mathrm{GR}}^{2}\right)$. Figure adapted from Nachstedt and Tetzlaff, (2017).

\section{Target Signal}

According to the n-back paradigm (Section 2.4.1), we define a target signal which depends on the comparison of the types (A or B) of the currently received input stimulus and of the stimulus received $n$ input pulses earlier (Figure 3.2, bottom left). The target signal is a one-dimensional signal for a single readout unit $\left(N_{R}=1\right)$. If the current stimulus is of the same type as the $\mathrm{n}^{\text {th }}$-last stimulus, the target signal is given by a positive pulse of the shape already described for input signals. This output pulse sets in with a delay $t_{\text {delay }}=25 \mathrm{~ms}$ after the onset of the current input pulse. If, in contrast, the current input stimulus is of a different type than the type of the $\mathrm{n}^{\text {th }}$-last stimulus, the target signal is given by a similar output pulse as in the first case but with a negative sign.

\section{Additional Readout Units}

In the second part of the analysis of the n-back performance of reservoir networks, we introduce $N_{\mathrm{A}}=2$ specially trained readout units to maintain the relevant information for solving the n-back task (Maass et al., 2007; Pascanu and Jaeger, 2011). These units reflect the types of the two last received input stimuli. In particular, the first additional readout unit is trained to have a value of 1 whenever the last received stimulus was of type $A$ and to attain a value of -1 whenever the last received stimulus was of type B. The second additional readout unit reflects the type of the second-last stimulus by a similar coding. Like the original target signal of the reservoir network n-back task, also the target signals of additional readout units react with a delay $t_{\text {delay }}=25 \mathrm{~ms}$ whenever a new input stimulus arrives. Furthermore, also the target signals for both additional readout units are smoothed by a convolution with a normalized Gaussian window with standard deviation $\sigma_{\text {smooth }}=5 \mathrm{~ms}$.

Formally, we define the additional readout units $A_{m}, m \in 1,2$, as

$$
A_{m}=\sum_{i=1}^{N_{\mathrm{G}}} w_{m i}^{\mathrm{AG}} F_{i}^{\mathrm{G}}
$$


with the trained synaptic weights $w_{m i}^{\mathrm{AG}}$. Feedback to the generator neurons is mediated by a weight matrix $W^{\mathrm{GA}}=\left(w_{i m}^{\mathrm{GA}}\right)$, with elements drawn from a normal distribution with zero mean and standard deviation $g_{\mathrm{GA}}$, and a corresponding additional term in Equation (3.2). During the training of the reservoir network using the ESN or the FORCE method, we treat these additional readout units completely identical to the original readout unit.

\section{Recall Stimulus}

In Section 3.2.5, we apply a recall stimulus to the network to trigger transient activity. This recall stimulus is modeled as an additional input signal to the network such that in this case, we have $N_{I}=3$. The recall stimulus is of similar shape as the input pulses representing the stimuli A or B. It is applied $25 \mathrm{~ms}$ before the onset of the target signal of the readout unit.

\subsubsection{Principal Component Analysis}

A complete analysis of the neuronal dynamics emerging in a reservoir network has to consider all $N_{\mathrm{G}}$ independent neuronal activities within the generator network. However, as proposed by Sussillo and Barak, (2013), projecting the reservoir dynamics onto its most relevant directions does already provide a good overview of the dynamics of the system. One established method to obtain such a projection is the principal component analysis (PCA; Wall et al., 2003). A PCA determines the linear uncorrelated directions within a set of high-dimensional samples and orders these directions according to the amount of variance occurring along them. These directions are referred to as the principal components of the system. They are given by the eigenvectors of the correlation matrix of the individual system variables. The corresponding eigenvalues, in turn, are proportional to the fraction of variance which is covered by the respective principal component.

For the analysis of the neuronal dynamics within the generator network, we sample the activity of the network while performing the n-back task for a certain amount of time steps. From the obtained samples, we determine the principal components by calculating the eigenvalues of the correlation matrix. Afterwards, we project the sampled dynamics onto the first two principal components, i.e. the two principal components with the highest eigenvalues. In the respective figures, we give the fraction of variance explained by the two principal components along the given axis.

\subsection{Performance of Reservoir Networks in the N-Back Task}

As discussed in Section 2.4.1, the n-back task is an established WM benchmark test. If neuronal networks relying on transient storage mechanism are a valid model of the neuronal system implementing WM, their performance in solving the n-back task should have similar characteristics as the performance observed in human subjects. Therefore, here, we study the performance of reservoir networks in solving the n-back task. We investigate the performance of reservoir networks trained with both the ESN and the FORCE algorithm to exclude training-algorithm specific effects.

We start by training reservoir networks to perform a regular n-back task with predictable stimulus timing. In a second step, we modify the n-back task similar to Koppe et al., (2014) by introducing unpredictable stimulus timing. Interestingly, we observe a break-down of the performance when gradually increasing the variance 
of the interstimulus intervals for both training methods. We demonstrate that the introduction of specially trained readout units, which assist the maintenance of the relevant information, restores the performance. In fact, we find that these additional readout units allow a level of robustness with respect to unpredictable stimulus timing which is comparable to the one observed in psychological experiments. In a final step, we investigate the dynamics of the original network and the one with additional specially trained readout units in the space spanned by the first two principal components. This investigation leads to an heuristic explanation of the observed effects.

\subsubsection{Predictable Stimulus Timing}

In a first step, we train reservoir networks consisting of $N_{G}=250$ neurons to solve an n-back task with $n=2$ in which the individual stimuli are presented with totally identical and hence predictable interstimulus intervals $\Delta t=0.2 \mathrm{~s}$ (compare Figure 3.2). Furthermore, we do not include feedback from the readout units to the generator network $\left(g_{\mathrm{GR}}=0\right)$. First, we study the results when training the network using the ESN method. In a second step, we employ the FORCE learning method for the same task.

When using the ESN method, the difference between the mean training error $E_{\text {train }}$ and the mean test error $E$ depends on the length of the training duration $t_{\text {train }}=s_{\text {train }} \cdot \Delta t_{\text {euler }}$. Here, $s_{\text {train }}$ is the number of time steps during which the reservoir dynamics is sampled during training and $\Delta t_{\text {euler }}$ denotes the time step of the numerical integration. If there is no feedback from the readout units to the neurons of the generator network, $E_{\text {train }}$ and $E$ converge toward a common value for long durations $t_{\text {train }}$ (Figure $3.3 \mathrm{a}$ ). The increase of $E_{\text {train }}$ with the parallel decrease of $E$ for longer learning durations $t_{\text {train }}$ reflects the transition of the readout weight matrix $W^{\mathrm{RG}}$ from a configuration specialized on the given training set to a configuration which generalizes across the presented input-output relation. The mean common convergence value is $\langle E\rangle \approx\left\langle E_{\text {train }}\right\rangle \approx 0.053$. Here, $\langle\cdot\rangle$ denotes the mean over 100 independent network instantiations. This low error value indicates a high capability of the network to perform the n-back task with predictable stimulus timing. This is confirmed by the visually close match between the target signal and the produced readout signal (Figure 3.3c). Hence, the ESN method is able to train a reservoir network to successfully perform the n-back task.

In contrast to the ESN method, the strength of the FORCE method is based on the fast adaptation of the readout weights to provide appropriate feedback signals. This implies that the FORCE method is not tailored for cases without feedback from the readout signals. As a result, the error $E$ of networks trained with the FORCE method does not converge as fast as a function of the training time $t_{\text {train }}$ as observed for the ESN method (Figure $3.3 \mathrm{~b}$ ). Still, after a training duration $t_{\text {train }}=10^{4} \mathrm{~s}$, the mean error of the readout signal is $\langle E\rangle \approx 0.065$. This error is only slightly larger than the average error obtained with the ESN method. Thus, also the FORCE method allows to train reservoir networks to perform the n-back task with predictable stimulus timing.

We conclude that both used training methods, ESN and FORCE learning, are able to optimize the readout weights of a reservoir network such as to achieve a high performance in the n-back task with predictable stimulus timing $\left(\sigma_{\Delta t}=0\right)$. The reservoir networks possess both the necessary short-term memory as well as the required computational power to perform this standard WM task with predictable temporal structure. In the following, we investigate the performance of reservoir networks in an $n$-back task with unpredictable stimulus timing. 

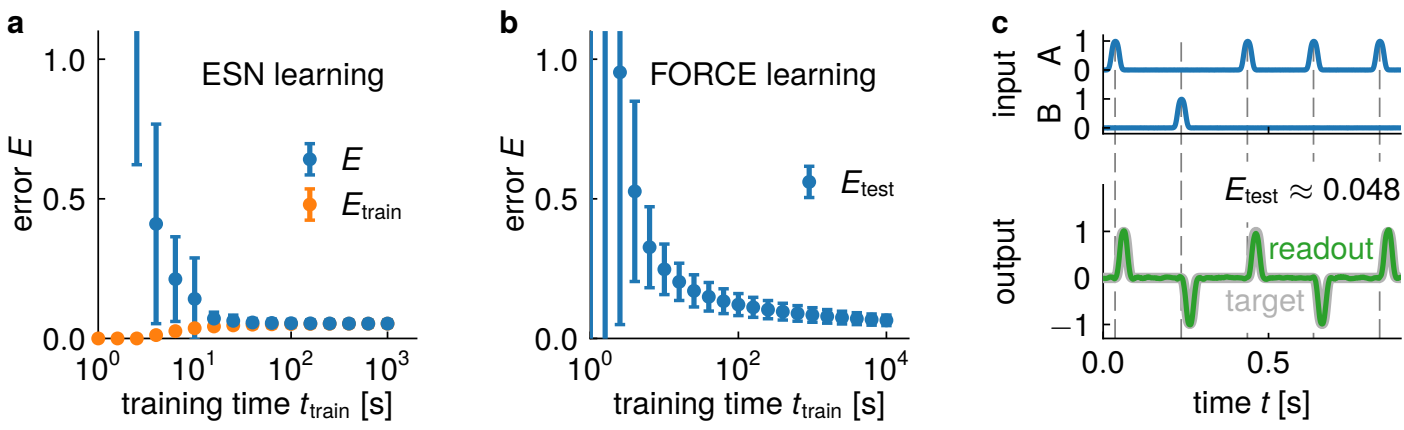

Figure 3.3: Training reservoir networks to perform the $\mathbf{n}$-back task. Reservoir networks are able to solve the $n$-back task with predictable stimulus timing. The constant interstimulus interval is $\Delta t=0.2 \mathrm{~s}$. The network parameters are $N_{\mathrm{G}}=250, g_{\mathrm{GG}}=1.0$, and $g_{\mathrm{GR}}=0$. (a) The mean training error $\left\langle E_{\text {train }}\right\rangle$ and the mean test error $\langle E\rangle$ when training reservoir networks to solve the n-back task using the ESN method converge toward a common small value $\left\langle E_{\text {train }}\right\rangle \approx\langle E\rangle \approx 0.053$ for long training sample durations $t_{\text {train }}=S_{\text {train }} \cdot \Delta t_{\text {euler }}$ (b) As the FORCE algorithm is an on-line method, there is no quantity like $E_{\text {train }}$ available when training reservoir networks using this method. After a training time $t_{\text {train }}=s_{\text {train }} \cdot \Delta t_{\text {euler }}=10^{4} \mathrm{~s}$, the mean test error in the $\mathrm{n}$-back task is $\langle E\rangle \approx 0.065$. The convergence is slower than for the ESN method. (c) The readout signal of a network which was trained using the ESN method and a training sample duration $t_{\text {train }}=1000 \mathrm{~s}$ corresponds almost perfectly to the target signal. The test error obtained for the complete test period $t_{\text {test }}$ in this reservoir network instantiation is $E \approx 0.048$. (a, b) Every data point represents the mean of 100 independent network instantiations. Error bars denote the standard deviation of the underlying distribution. Note the logarithmic scale of the $t_{\text {train }}$-axis.

\subsubsection{Unpredictable Stimulus Timing}

As we discussed in Section 2.4.1, experimental results show that the performance of human subjects while performing different variants of the n-back task does not decrease when the stimuli are presented with an unpredictable timing. If the reservoir networks investigated here are a plausible model of WM, they should show a comparable tolerance to variances in the input timing.

Before introducing unpredictability into the timing of the input stimuli by mixing different interstimulus intervals, however, we first investigate the general influence of different interstimulus intervals $\Delta t$ on the reservoir performance in the n-back task. For both learning methods, longer intervals $\Delta t$ lead to an increased average error $\langle E\rangle$ (Figures $3.4 \mathrm{a}$ and $3.4 \mathrm{c}$ ). This is because the activity of the network decays closer to the noise level in between the different stimuli for larger values of $\Delta t$. Note, however, that the mean error is below 0.5 for all interstimulus intervals up to $500 \mathrm{~ms}$. This is of relevance in the following, when a distribution of interstimulus intervals is employed.

We introduce unpredictability into the stimulus timing by drawing the individual interstimulus intervals $\Delta t_{i}$ from a normal distribution with mean $\mu_{\Delta t}$ and standard deviation $\sigma_{\Delta t}$. Note that we apply this distribution both during the training and the testing phase of the reservoir network. Thus, for every value of the standard deviation $\sigma_{\Delta t}$, a new set of readout weights is trained which is optimized for the specific value of $\sigma_{\Delta t}$. Interestingly, the reservoir networks turn out to be significantly more vulnerable to variances in the stimulus timing than it is found in WM experiments. For the ESN learning approach, a standard deviation of $\sigma_{\Delta t}=50 \mathrm{~ms}$ results in a mean error of $\langle E\rangle \approx 0.74 \pm 0.02$ (Figure $3.4 \mathrm{~b}$ ). Note that next to all interstimulus intervals which are expected to be drawn from a normal distribution with mean $\mu_{\Delta t}=200 \mathrm{~ms}$ and standard deviation $\sigma_{\Delta t}=50 \mathrm{~ms}$ result in significantly lower errors in the case of predictable stimulus timing (green distribution in Figure $3.4 \mathrm{a}$ ). Thus, the increase 

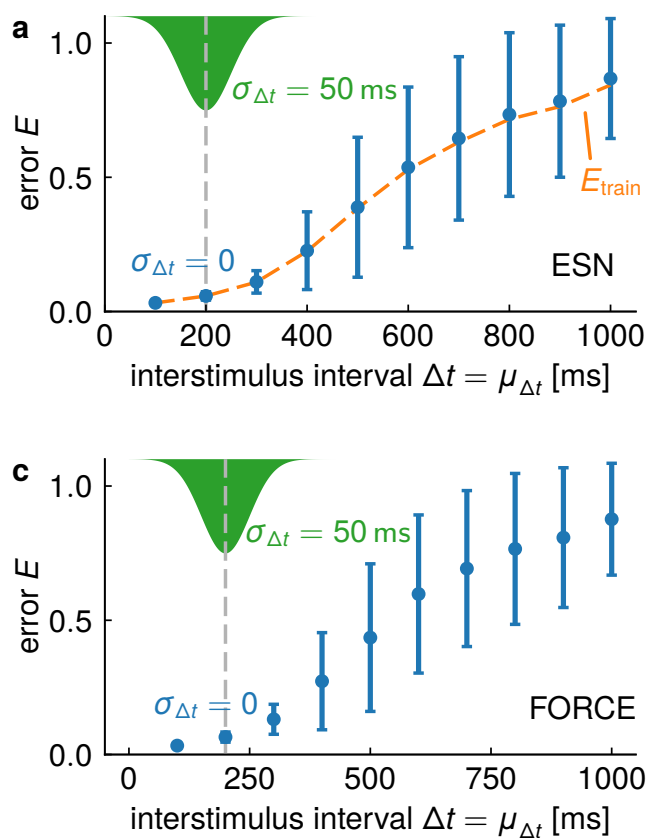
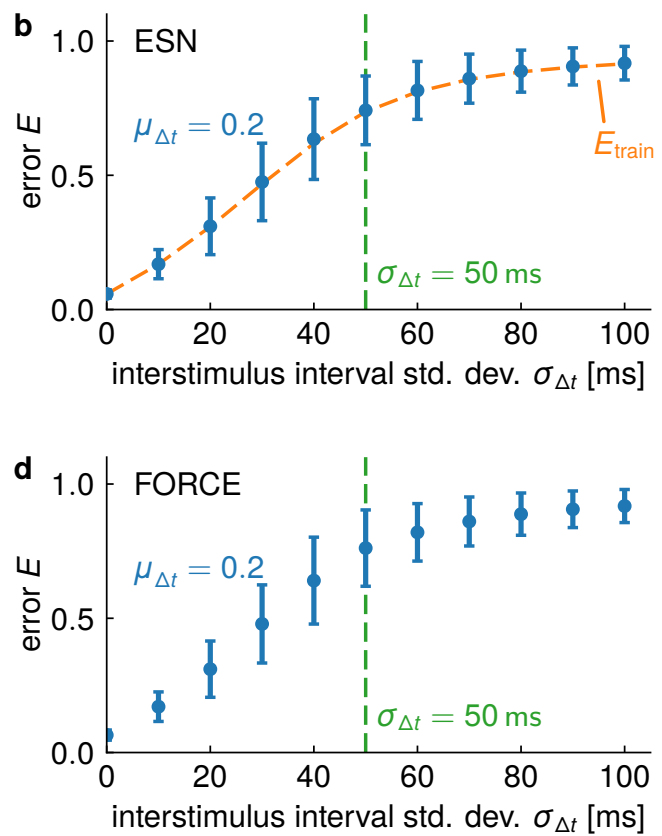

Figure 3.4: Influence of the interstimulus interval and of temporal unpredictability on the nback performance of reservoir networks. The error $E$ of reservoir networks solving the $n$-back task increases for larger (but constant) interstimulus intervals $\Delta t$ as well as for increased unpredictability of the stimulus timing as expressed by the standard deviation $\sigma_{\Delta t}$. (a) We train reservoir networks using the ESN method to solve $n$-back tasks with perfectly predictable temporal structure $\left(\sigma_{\Delta t}=0 \mathrm{~ms}\right)$. The mean error $\langle E\rangle$ increases for longer interstimulus intervals $\Delta t$. The green distribution at the top visualizes a normal distribution of interstimulus intervals with mean $\mu_{\Delta t}=200 \mathrm{~ms}$ (dashed gray line) and standard deviation $\sigma_{\Delta t}=50 \mathrm{~ms}$. Note that for all values of $\Delta t$ with relevant probability in this distribution, the mean error is well below 0.5. (b) Using again the ESN method, we train reservoir networks to solve the n-back task with a mean interstimulus interval $\mu_{\Delta t}=200 \mathrm{~ms}$ (dashed gray line in (a)) and varying standard deviation $\sigma_{\Delta t}$ of the distribution of interstimulus intervals. The error increases for larger values of $\sigma_{\Delta t}$. Importantly, for a standard deviation $\sigma_{\Delta t}=50 \mathrm{~ms}$ (green line), the mean error is well above 0.5 . This is significantly larger than for almost all constant values of $\Delta t$ which are expected to occur in the respective distribution (green distribution in (a)). (c, d) The results obtained using the FORCE method correspond to the results for the ESN method. This is expected in the case without feedback signals from the readout neurons back to the generator neurons $\left(g_{\mathrm{RG}}=0\right)$. (a-d) In all cases, we train the network on the same interstimulus interval statistics than used during testing. There is no feedback from the readout units to the generator network $\left(g_{\mathrm{RG}}=0\right)$. The data points represent the mean of 100 independent network instantiations. The error bars are given by the standard deviation of the underlying distribution of error values. (a, b) The training sample duration during the ESN learning is $t_{\text {train }}=10^{3} \mathrm{~s}$. The dashed orange line shows the mean $\left\langle E_{\text {train }}\right\rangle$ of the training error as a function of $\Delta t$. (c, $\left.\mathbf{d}\right)$ The reservoir networks are trained using the FORCE method for a duration of $t_{\text {train }}=10^{4} \mathrm{~s}$ and $\alpha=10^{-3}$. 
of the error as compared to the completely predictable n-back task ( $\left.\sigma_{\Delta t}=0 \mathrm{~ms}\right)$ cannot be solely explained by the fact that larger interstimulus intervals occur in the unpredictable case.

Training the networks using the FORCE algorithm, we find a very similar relation between the standard deviation $\sigma_{\Delta t}$ and the error $E$ (Figure 3.4 d). For a standard deviation of $\sigma_{\Delta t}=50 \mathrm{~ms}$, the mean error is $\langle E\rangle \approx 0.76 \pm 0.02$. Also here, this error is higher than what we found in the case of predictable stimulus timing for next to all interstimulus intervals which are drawn from the respective distribution (green distribution in Figure 3.4c). Having observed the increase of the error for both the ESN and the FORCE training method, we conclude that the break-down of performance in the presence of unpredictable input timing is independent of the used training algorithm.

Even though the vulnerability with respect to unpredictable stimulus timing is independent from the employed learning algorithm, it might still be related to the specific choice of reservoir parameters. Therefore, in the following, we investigate the stability of this finding when varying different parameters of the network architecture.

At first, we study the influence of the standard deviation $g_{\mathrm{GR}}$ of the weights projecting the readout signal back to the neurons of the generator network (Figure $3.5 \mathrm{a}$ and Figure $3.5 \mathrm{~b}$ ). As it turns out, introducing feedback via non-zero values of $g_{G R}$ does not support but rather weaken the performance of reservoir networks when performing the n-back task. This is more pronounced in the case of the ESN method (3.5 a) which has to rely on feedback-clamping during training to deal with these kind of connections (compare Section 3.1.2). However, also for the FORCE method (Figure $3.5 \mathrm{~b}$ ), no significant improvement of the performance can be observed for stronger feedback connections. Even though, the increase of the error for higher values $g_{\mathrm{GR}}$ is not as pronounced as in the ESN case. This reflects the superiority of the FORCE method in the presence of feedback connections as compared to the ESN method.

In a next step, we analyze the influence of the number of neurons $N_{G}$ in the generator network on the performance of the reservoir networks in the n-back task with unpredictable stimulus timing. Here, the qualitative results for the ESN learning method (Figure 3.5c) and the FORCE learning method (Figure 3.5d) are similar. In general, increasing $N_{G}$ decreases the average error $\langle E\rangle$ for a given value of $\sigma_{\Delta t}$. Nevertheless, the increase of $E$ for large values of $\sigma_{\Delta t}$ remains qualitatively similar. Basically, the error curve is shifted toward higher values of $\sigma_{\Delta t}$. Given that the tested values of $\sigma_{\Delta t}$ are comparably small and the related improvement of the tolerance with respect to timing variance is weak, this implies a very high number of neurons needed to solve this rather simple WM task. Thus, we conclude that a high number $N_{\mathrm{G}}$ of generator neurons may not overcome the problem of decreased performance in the n-back task with unpredictable stimulus timing.

Finally, we observe that also modifying the gain parameter $g_{\mathrm{GG}}$ of the distribution of weights within the generator network does not reduce the vulnerability of the reservoir network with respect to unpredictable stimulus timing in the n-back task (Figure 3.5 e and Figure $3.5 \mathrm{f}$ ). For both training methods, decreasing $g_{\mathrm{GG}}$ to a smaller value $\left(g_{\mathrm{GG}}=0.9\right)$ or increasing it to a larger value $\left(g_{\mathrm{GG}}=1.1\right)$ than the used value slightly below the edge of chaos $\left(g_{\mathrm{GG}}=1.0\right)$ leads to an increase of the error $E$ for most tested values of $\sigma_{\Delta t}$.

Thus, neither varying the standard deviation $g_{\mathrm{GR}}$ of the feedback connections from the readout units to the neurons in the generator network (Figures $3.5 \mathrm{a}$ and $3.5 \mathrm{~b}$ ) nor increasing or decreasing the number $N_{\mathrm{G}}$ of neurons within the generator network (Figures $3.5 \mathrm{c}$ and $3.5 \mathrm{~d}$ ) nor trying different gain parameters $g_{\mathrm{GG}}$ of the internal synaptic weights within the generator network (Figures $3.5 \mathrm{e}$ and $3.5 \mathrm{f}$ ) challenges the 

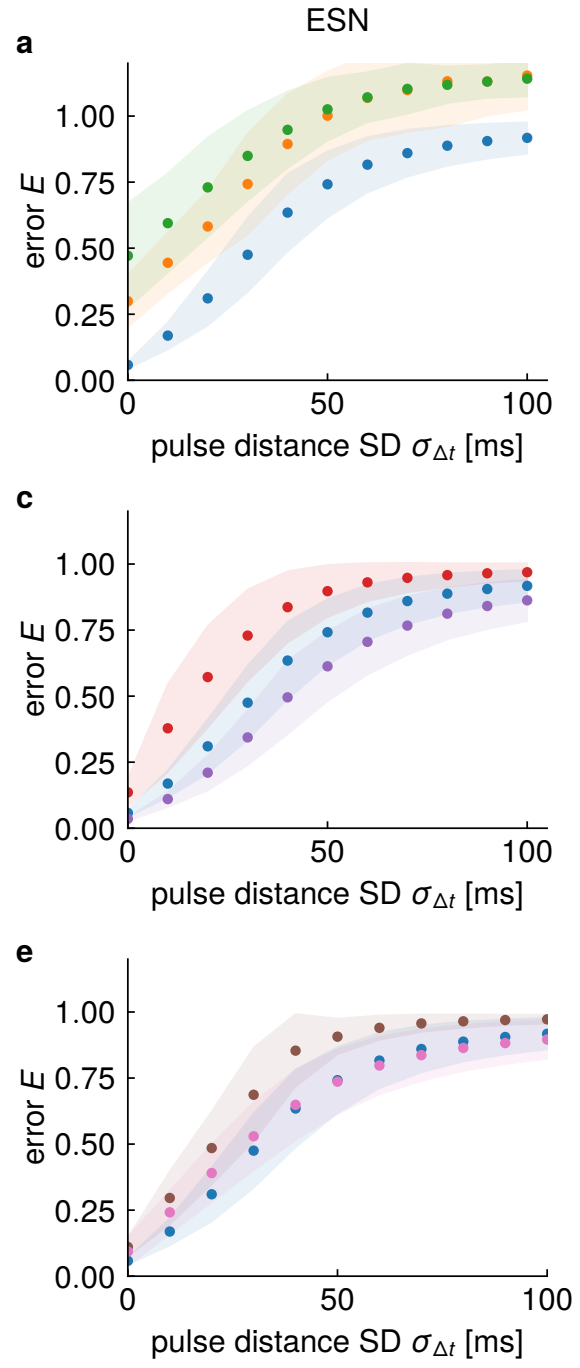

b

- $g_{\mathrm{GR}}=0.0$

- $g_{\mathrm{GR}}=0.1$

- $g_{\mathrm{GR}}=0.2$

$N_{\mathrm{g}}=250$

$g_{\mathrm{GG}}=1.0$

FORCE

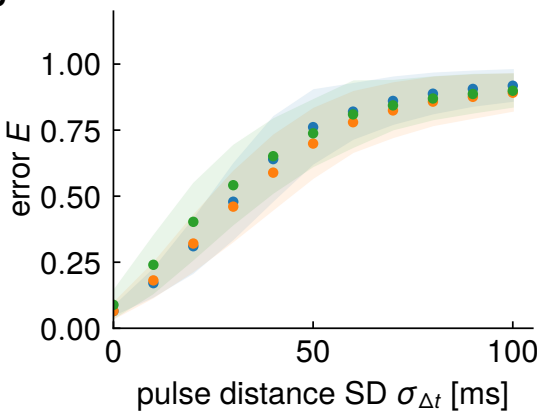

d

- $N_{G}=100$

- $N_{G}=250$

- $N_{G}=350$

$g_{\mathrm{GR}}=0.0$

$g_{\mathrm{GG}}=1.0$

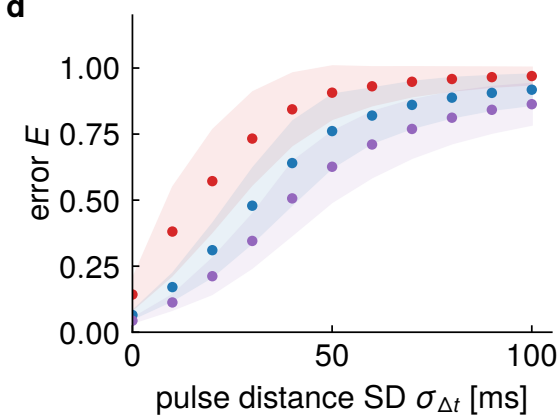

f

- $g_{\mathrm{GG}}=0.9$

- $g_{\mathrm{GG}}=1.0$

- $g_{\mathrm{GG}}=1.1$

$g_{\mathrm{GR}}=0.0$

$N_{\mathrm{G}}=250$

Figure 3.5: Influence of different network parameters on the performance of reservoir networks in the $\mathbf{n}$-back task with unpredictable stimulus timing. The error $E$ of the output signal in the $\mathrm{n}$-back task with $\mu_{\Delta t}=200 \mathrm{~ms}$ increases for larger standard deviations $\sigma_{\Delta t}$ of the interstimulus intervals $\Delta t$. This finding is independent of the used network parameters. (a, b) We train networks with different strengths of the feedback connections from the readout units back to the generator network as expressed by the standard deviation $g_{\mathrm{GR}}$ of the respective weight matrix. For both training methods (ESN and FORCE), increasing $g_{\mathrm{GR}}$ leads to increased errors $E$ for a given value of $\sigma_{\Delta t}$. Still, the FORCE methods achieves lower error values than the ESN method for nonzero $g_{\mathrm{GR}}$ values. The number $N_{\mathrm{G}}=250$ of neurons in the generator network and the gain parameter $g_{\mathrm{GG}}=1.0$ in the respective weight matrix are identical for all network instantiations. (c, d) Increasing the number $N_{G}$ of neurons in the generator network reduces the error $E$ for a given value of $\sigma_{\Delta t}$. Nevertheless, qualitatively, the performance of the networks shows the same strong vulnerability to unpredictable stimulus timing, i.e., large values of $\sigma_{\Delta t}$. The constant parameters are $g_{\mathrm{GR}}=0$ and $g_{\mathrm{GG}}=1.0$. (e, f) When varying the gain parameter $g_{\mathrm{GG}}$ of the weight matrix within the generator network, the lowest error $E$ for most values of $\sigma_{\Delta t}$ is obtained for $g_{\mathrm{GG}}=1.0$, i.e., slightly below the edge of chaos (compare Section 3.1.1). Neither decreasing nor increasing $g_{\mathrm{GG}}$ improves the robustness of the network performance with respect to unpredictable stimulus timing. (a-f) Every data point represents the mean error of 100 independent network instantiations. The shaded areas show the standard deviation of the respective distributions. (a, c, e) The training sample duration during the ESN learning is $t_{\text {train }}=10^{3} \mathrm{~s}$. (b, d, f) The FORCE learning parameters are $t_{\text {train }}=10^{4} \mathrm{~s}$ and $\alpha=10^{-3}$. Figure adapted from Nachstedt and Tetzlaff, (2017). 
described qualitative finding. Independent of the used network parameters and of the employed learning algorithm, reservoir networks show a low performance in the n-back task with unpredictable stimulus timing. As this contradicts the described results from psychological experiments which do not find an influence of stimulus timing unpredictability on the performance in the n-back task (Koppe et al., 2014), we can exclude reservoir networks with purely transient dynamics as a plausible model of WM.

\subsubsection{Restoring Performance with Additional Readout Units}

In the previous section, we observed that regular reservoir networks fail in the n-back task with unpredictable stimulus timing. This indicates that in these systems, the relevant information about past stimuli is represented in a way that strongly relies on precisely timed input stimuli. As a result, if the input stimuli occur unpredictably, the relevant information cannot be reliably read out to produce a time-dependent output signal as required in the n-back task. Therefore, here, we modify the training of the reservoir networks to ensure that the relevant information about past stimuli is kept in a robust manner. A possible way to obtain such a robust representation is by introducing $N_{\mathrm{A}}=2$ additional specially trained readout units whose output values reflect the stored information in a straight-forward way (compare Section 3.1.3 and Maass et al., 2007; Pascanu and Jaeger, 2011). The output of the first additional readout unit is always positive if the last received stimulus was of type $A$ and negative if it was of type $\mathrm{B}$. The second additional readout units remembers the type of the second-last received stimulus in an identical manner. The signal of the additional readout units are fed back via random connections into the generator network where they influence the ongoing dynamics based on the remembered information (Figure 3.6). Thus, in some sense, the idea is to lift the network up by its own bootstraps. Both additional readout units are trained by the same mechanisms and in parallel to the regular readout unit.

In order to evaluate whether this adapted reservoir training schema achieves better performances in the n-back task with unpredictable stimulus timing, various instances of reservoir networks with the same parameters as used before are trained on the modified task including the additional readout units. As above, for both the ESN approach and the FORCE learning method, the level of unpredictability of the stimulus timing as expressed by the standard deviation $\sigma_{\Delta t}$ of the underlying distribution is varied. According to the observation that feedback from the regular readout unit back to the reservoir network does not support the operation in the n-back task, we always set $g_{\mathrm{GR}}=0$.

We find that for both training methods, the standard deviation $g_{\mathrm{GA}}$ of the feedback weights from the additional readout units to the generator network determines the susceptibility of the n-back performance to unpredictable stimulus timing (Figures $3.7 \mathrm{a}$ and $3.7 \mathrm{~b}$ ). For very low values of $g_{\mathrm{GA}}$, higher standard deviations $\sigma_{\Delta t}$ still lead to an increase of the error $E$ until the saturation level near $E \approx 1.0$ is reached. If $g_{\mathrm{GA}}$ is chosen as a lager value, this saturation level decreases. For $g_{\mathrm{GA}}=1.0$, the error $E$ is low for all values of the timing standard deviation $\sigma_{\Delta t}$ or, in other words, no longer dependent on $\sigma_{\Delta t}$. Thus, adding the additional readout units which reflect and maintain the relevant information of past stimuli for the n-back task leads to a significant improvement of the performance of reservoir networks in the n-back task with unpredictable stimulus timing. The robustness of the system with respect to the stimulus timing is now compatible with the one found for human subjects performing the n-back task (Koppe et al., 2014). In the following, we investigate the 


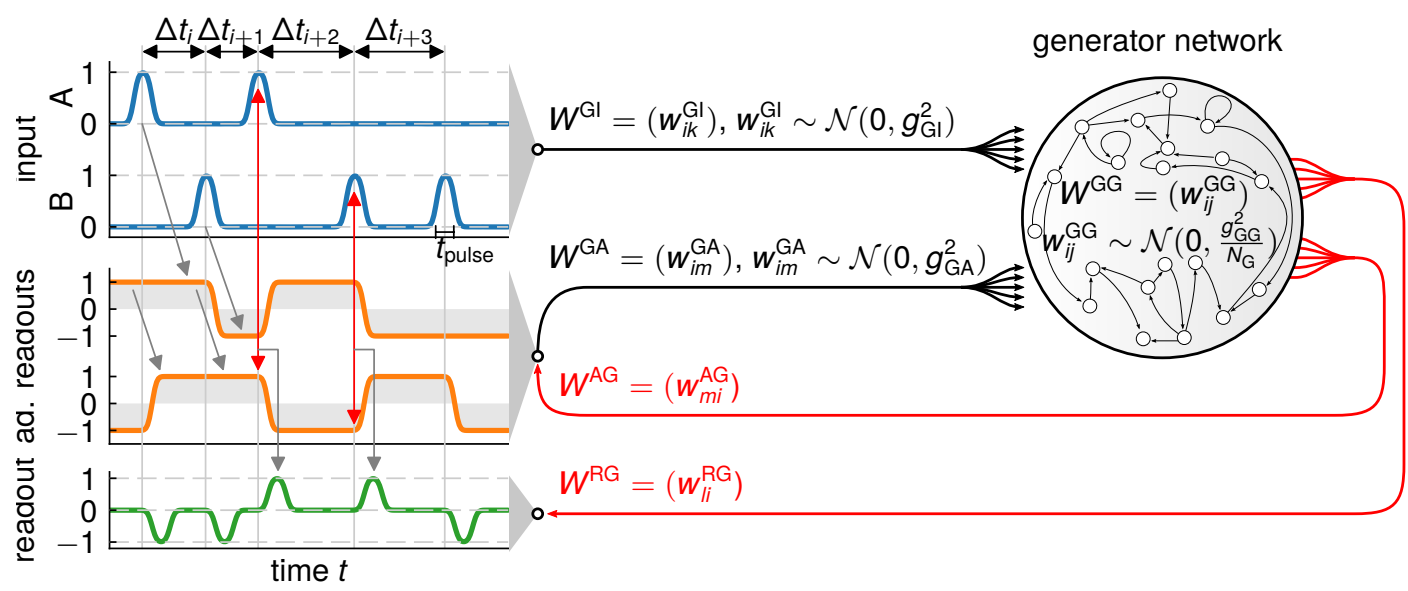

Figure 3.6: Setup for the reservoir $n$-back task with additional readout units. The input signal and the target signal as well as the related connectivity are identical to the setup without additional readout units (Figure 3.2). $N_{\mathrm{A}}=2$ additional readout neurons are introduced whose target signals reflect the identity of the two last received input stimuli. One of them (upper orange trace) attains a value of 1 when the last received stimulus was an "A". If the last received stimulus was a "B", the additional readout signal is supposed to attain a value of -1 . The target signal for the second additional readout unit (lower orange trace) contains the same information about the second-last stimulus. These two additional readout signals are projected back to the generator network via a synaptic weight matrix $W^{\mathrm{GA}}$ with elements $w_{i m}^{\mathrm{GA}}$ drawn from a normal distribution with zero mean and standard deviation $g_{\mathrm{GA}}$. Feedback from the original readout unit back to the generator network is neglected $\left(g_{\mathrm{GR}}=0\right)$. Figure adapted from Nachstedt and Tetzlaff, (2017).

dynamical cause of this qualitative change by projecting the reservoir dynamics into the space spanned by its principal components.

\subsubsection{Dynamics of Principal Components}

The qualitative performance difference in the n-back task with unpredictable stimulus timing between reservoir networks with and without the additional readout units indicates a qualitative difference in the underlying dynamics. Instead of analyzing the dynamics of the different reservoir networks in the complete high-dimensional activity space, we project the activities within the generator network onto its two most significant principal components (Section 3.1.4). This allows for an heuristic understanding of the basic dynamical modes that determine the dynamics of the respective reservoir networks (Sussillo and Barak, 2013).

At first, we investigate the dynamics of a representative example of a reservoir network without any additional readout units which operates on an n-back task with constant interstimulus intervals $\left(\sigma_{\Delta t}=0 \mathrm{~ms}\right.$, Figure $\left.3.8 \mathrm{a}\right)$. The dynamics of the network is dominated by a single attractor state at zero activity. Thus, the reservoir network indeed operates on purely transient dynamics. When the network is exposed to an ongoing stream of stimuli like in the n-back task, the attractor state is never reached. Instead, the incoming stimuli keep perturbing the system away from its convergence toward the attractor. Importantly, the state of the system at the occurrence of a stimulus depends on the earlier received stimuli. This results in transient trajectories which are not only characteristic for the recently received input stimulus but also for the history of the earlier received stimuli. For instance, there is a specific bundle of trajectories representing states in which the network has received an "A" stimulus followed by two "B" stimuli $\left(*_{1} \rightarrow *_{2} \rightarrow *_{3}\right.$ in Figure 3.8 a). As a result, trajectories during which the network has to produce a positive output pulse 

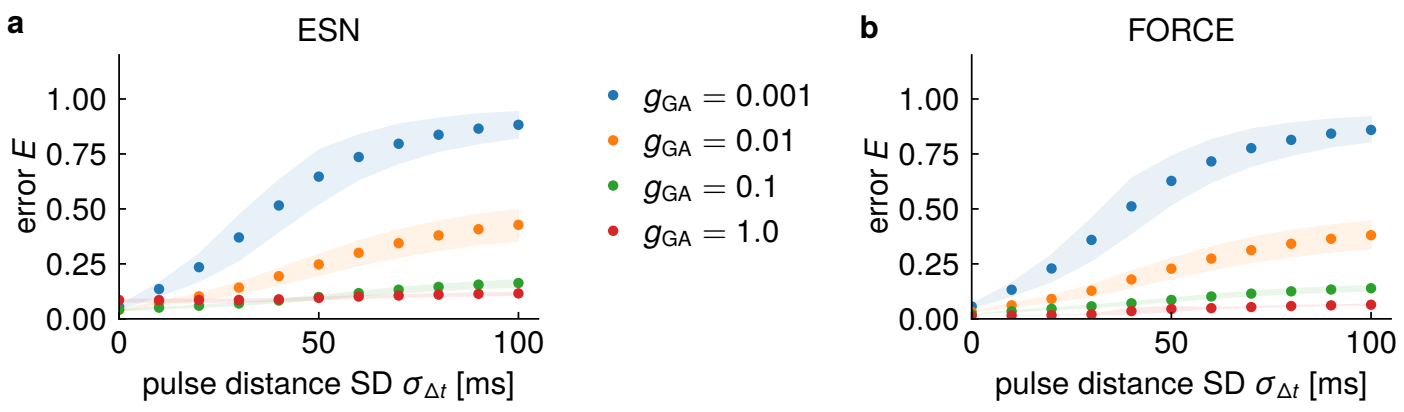

Figure 3.7: Performance of reservoir networks with additional readout units in the n-back task with unpredictable stimulus timing. In contrast to the case without additional readout units (Figure 3.5), reservoir networks with strong feedback from these additional readout units are able to solve the n-back task also for highly unpredictable input stimulus timing. For stronger feedback from the additional readout units, i.e. for larger values of $g_{\mathrm{GA}}$, the error $E$ gets smaller also for large values of the standard deviation $\sigma_{\Delta t}$ of the interstimulus intervals. Thus, the performance becomes independent from the predictability of the temporal structure of the $n$-back task. These results are confirmed by both training methods (ESN and FORCE). (a) The training sample duration during the $E S N$ learning is $t_{\text {train }}=10^{3} \mathrm{~s}$. (b) The FORCE learning parameters are $t_{\text {train }}=10^{4} \mathrm{~s}$ and $\alpha=10^{-3}$. Figure adapted from Nachstedt and Tetzlaff, (2017).

and trajectories during which a negative output pulse is required are close but can be clearly separated (red and blue trajectories in Figure 3.8 a). Thus, all the relevant information required for the $n$-back task is stored in distinguishable trajectories of the network dynamics.

Introducing unpredictability (here, $\sigma_{\Delta t}=100 \mathrm{~ms}$ ) in the timing of the input stimuli directly affects this transient storage mechanism of the network without additional readout units (Figure $3.8 \mathrm{~b}$ ). The incoming stimuli do now appear at different stages of the convergence of the system dynamics toward the single attractor state. As a result, the trajectory bundles representing different stimulus histories become broader. The same sequence of stimuli may result in very different trajectories in the phase space. For large value of $\sigma_{\Delta t}$, the trajectories requiring a positive output signal can no longer be reliably separated from the trajectories requiring a negative output signal. Thus, the readout unit is no longer able to extract the relevant information for the n-back task and to produce the required time-dependent readout signal.

When we add the additional readout neurons to the network, this results in a significant change of the system dynamics (Figure 3.8c). Now, the network possesses four distinct attractor states with each of them corresponding to one specific combination of the last two received stimuli. Whenever a new stimulus arrives, it pushes the system along a characteristic transient trajectory which interlinks the different attractor states. These transient dynamics are used to process the incoming information and to produce the desired time-dependent readout signals. As before, a reservoir network which receives an " $\mathrm{A}$ " stimulus followed by two " $\mathrm{B}$ " stimuli evolves along a set of characteristic trajectories $\left(*_{1}, *_{2}, *_{3}\right.$ in Figure $\left.3.8 \mathrm{c}\right)$. In contrast to the case without additional readout units, however, these trajectories do now interlink history-specific attractor states $\left(\diamond_{1}, \diamond_{2}\right)$. Thus, the information about the type of the recent stimuli is stored in the attractor states while the transient trajectories in between these states are exploited for reading out the desired output signal.

In contrast to the original reservoir network, the dynamics of the reservoir network with additional readout units is robust with respect to unpredictable stimulus timing in the n-back task. Increasing the standard deviation $\sigma_{\Delta t}$ of the interstimulus intervals (here, $\sigma_{\Delta t}=100 \mathrm{~ms}$ ) does not result in significant overlaps of the different trajectories 
$\sigma_{\Delta t}=0 \mathrm{~ms}$

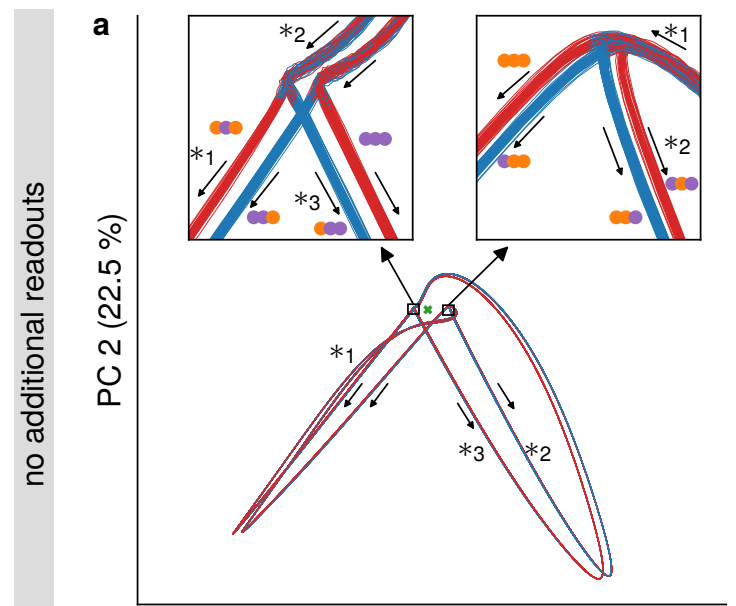

PC $1(37.9 \%)$

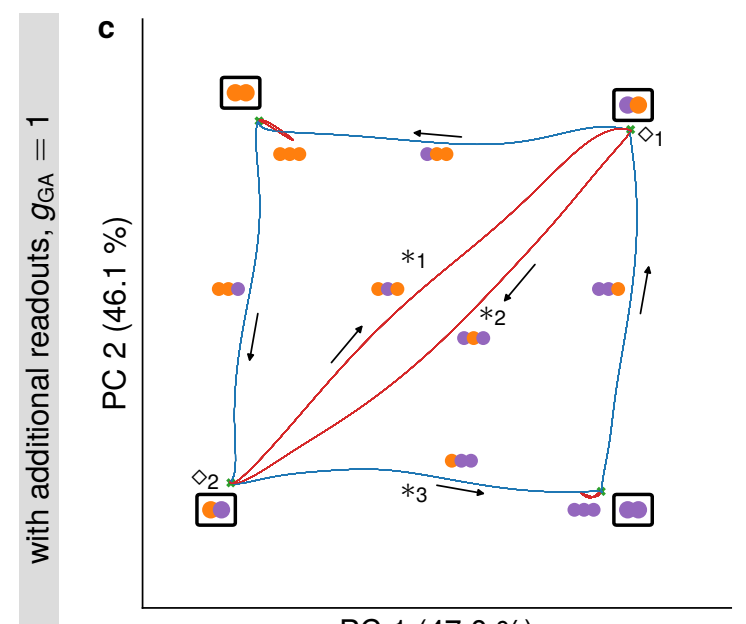

PC $1(47.0 \%)$
$\sigma_{\Delta t}=100 \mathrm{~ms}$

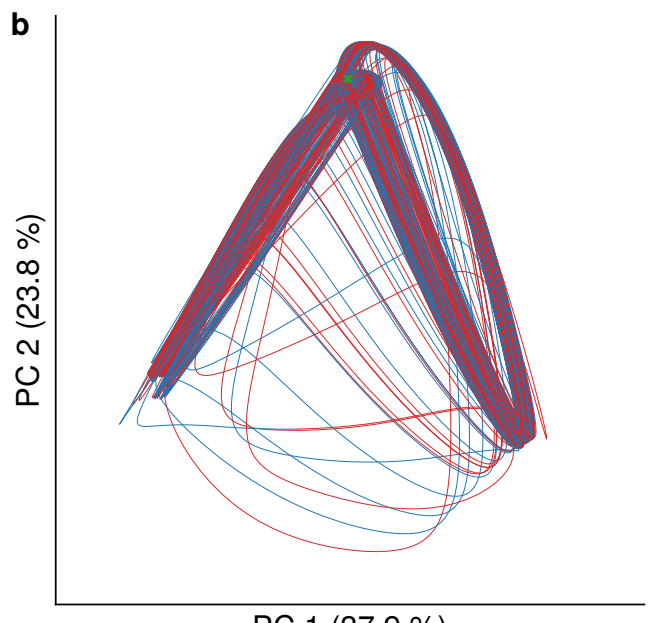

PC $1(37.9 \%)$

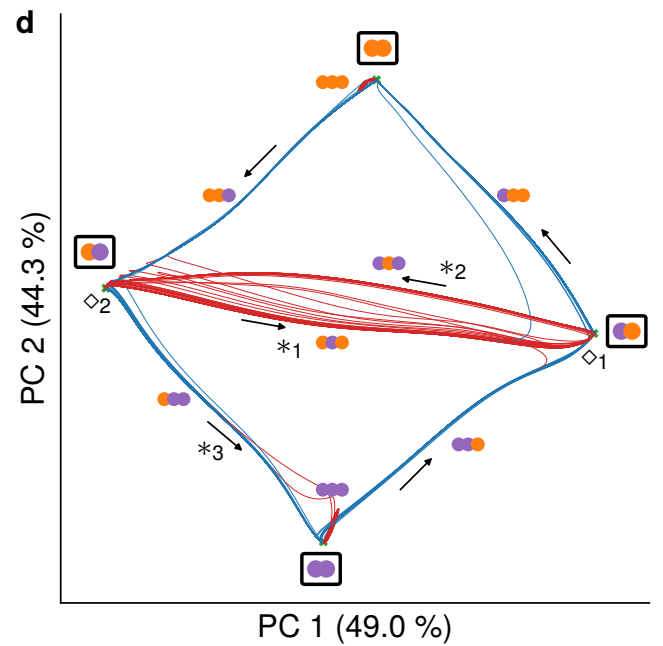

— negative output pulse required positive output pulse required
* stable equilibrium / attractor

- traversal direction

three most recent stimuli (second last, last, current):

$\begin{array}{llllll}A A A A & \text { ABA } & \text { BAA } & \square B A & \square B A \\ \text { AAB } & \text { ABB } & \text { BAB } & \text { B B } & \square A B & \square B B\end{array}$

Figure 3.8: Dynamics of a reservoir network during performing the $n$-back task projected onto the first two principal components. The color of the different trajectories encodes the sign of the output pulse that has to be produced along the given trajectory. The percentage values at the axes give the fraction of variance explained by the respective principal component. (a) The reservoir network without additional readout units stores the information about the current stimulus history in distinct transient trajectory bundles. (b) When we introduce unpredictable stimulus timing, these trajectory bundles overlap. This impedes a proper readout of the desired signals. (c) The addition of specific additional readout units "restructures" the dynamics of the system by introducing several distinct attractor states. These attractor states store the information about the past stimuli. (d) Even in the case of unpredictable stimulus timing, the attractor states introduced by the additional readout units preserve the difference between the different trajectory bundles. This enables a proper readout of the desired signals. (a-d) In all cases, the mean interstimulus interval is $\mu_{\Delta t}=200 \mathrm{~ms}$. There is no feedback from the original readout units $\left(g_{\mathrm{GR}}=0\right)$ and, if applicable, strong feedback from the additional readout units $\left(g_{\mathrm{GA}}=1\right)$. The networks are trained using the FORCE method with $t_{\text {learn }}=10^{4} \mathrm{~s}$ and $\alpha=10^{-3}$. Figure adapted from Nachstedt and Tetzlaff, (2017). 
a without recall stimulus

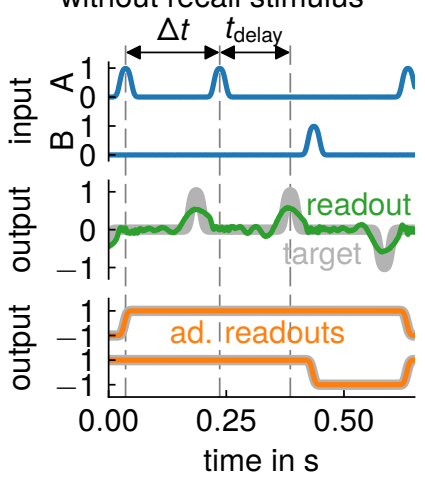

b with recall stimulus

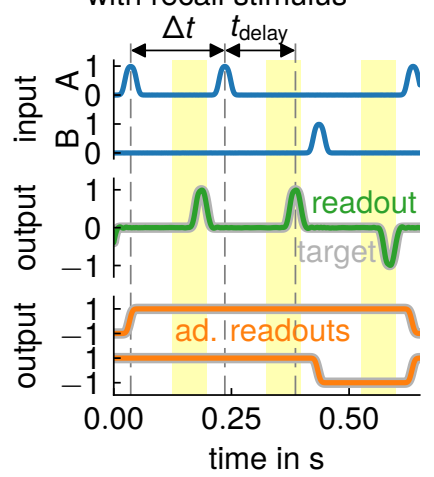

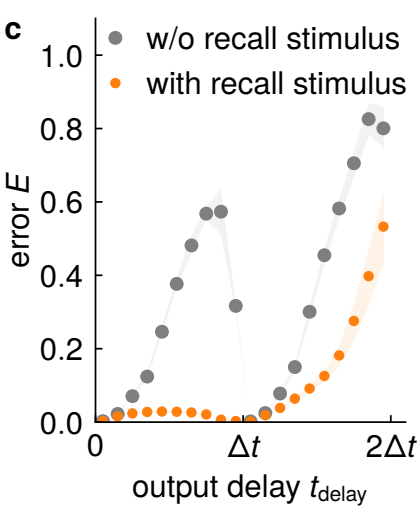

Figure 3.9: Performance of a reservoir network with additional readout units in n-back tasks with different response delays. We train reservoir networks with two additional readout units to perform the $n$-back task with $n=1$ and an increased output delay $t_{\text {delay }}$. (a) Interestingly, the reservoir network is only able to produce a readout signals (green) with significant differences to the target signal (gray). The pure information about the type of the past stimuli can still be reliably read out (orange). (b) Introducing an unspecific additional recall stimulus which perturbs the system shortly before the onset of the output pulse restores the performance. (c) Without an additional recall stimulus (gray dots), the error $E$ of the readout signal increases with larger output delays $t_{\text {delay }}<\Delta t$ as the system reaches an attractor state. The occurrence of the next input stimulus leads to a new transient trajectory which again enables the system to produce the time-dependent output signal. If an additional recall stimulus is introduced (orange dots), the error $E$ decreases for all output delays. For $t_{\text {delay }}<\Delta t$, the recall stimulus initializes a transient trajectory which can be used to read out a time-dependent signal based on the information stored in the attractor state. For $t_{\text {delay }}>\Delta t$, the effect diminishes as the relevant information is no longer available in the currently active attractor state. Figure adapted from Nachstedt and Tetzlaff, (2017).

(Figure $3.8 \mathrm{~d}$ ). Instead, the trajectories remain clearly separated. This enables the readout unit to extract the relevant information and to produce the desired timedependent output signal.

In summary, introducing the additional readout neurons into the reservoir network results in a set of distinct attractor states which "structure" the phase space. As a consequence, also the trajectories requiring different output signals remain clearly separated in the case of unpredictable stimulus timing. This separation of the trajectories reflects the dynamical cause of the robust performance of the network with additional readout units in the n-back task with unpredictable stimulus timing.

\subsubsection{Transient Dynamics and the Generation of Time-Dependent Signals}

As demonstrated, only the introduction of distinct attractor states enables a reservoir network to solve the n-back task with unpredictable stimulus timing. While this emphasizes the relevance of attractor states for robust WM operation, it does not diminish the importance of the transient dynamics for WM. In fact, only the transient trajectories of the system enable the readout unit to produce a time-dependent (nonconstant) output signal. This includes tasks during which the information stored in WM has to be read out only at specific periods of time.

By varying the time interval $t_{\text {delay }}$ in between the input stimulus and the respective output pulse in the target signal, this yields an interesting prediction. As before, we consider a reservoir network with two additional readout units. However, now, this network is performing an n-back task with $n=1$. This means it has to compare the type of the current stimulus with the type of the previous stimulus. Furthermore, we assume a completely predictable stimulus timing $\left(\sigma_{\Delta t}=0\right)$. According to our earlier results, the reservoir network is able to solve this task with only a small error $E$ for 
short time intervals $t_{\text {delay }}$ (gray dots in Figure $3.9 \mathrm{c}$ ). Here, the network is still in the transient state which is evoked by the recently received input stimulus. When we increase the delay $t_{\text {delay, }}$, the system dynamics gets closer to the attractor state and the complexity of the transient dynamics decreases. As a result, the time-dependent output signal can no longer be read out precisely and the error $E$ of the readout signal increases (Figure 3.9 a). Note that the pure information about the type of the two last stimuli can still be easily read out as demonstrated by the two additional readout units. Without transient dynamics, however, this information cannot be used to generate a time-dependent signal. Consequently, for $t_{\text {delay }}>\Delta t$, the occurrence of the next input stimulus leads to new complex transient dynamics and thereby again enables the reservoir network to produce the time-dependent output signal. Based on this observation, we introduce an additional recall stimulus which we always apply shortly before the onset of an output pulse. This additional input signal restores the performance of the reservoir network for time delays $0<t_{\text {delay }}<\Delta t$ by pushing the dynamics of the network back into a transient state (orange dots in Fig $3.9 \mathrm{~b}$ ). Importantly, this recall stimulus does not convey task-relevant information. Its only role is to trigger complex transient dynamics that may be used to readout the stored information in a time-dependent way. Therefore, this does only work as long as the relevant information is still available in the network. Once the current attractor state does no longer contain the necessary information to produce the current output pulse, the enhancing effect of the recall stimulus vanishes ( $t_{\text {delay }}>\Delta t$ in Figure $\left.3.9 \mathrm{c}\right)$.

These results show that both the attractor states as well as the transient dynamics in between them have to be exploited in order to obtain a robust and functional network model of working memory. In the presence of unpredictable stimulus timing, information can only be reliably stored in attractor states. Information processing in terms of a temporally specific readout signals, on the other hand, requires transient dynamics.

\subsection{Discussion}

In this chapter, we address the still unresolved question regarding the type of neuronal network dynamics underlying the operation of WM. Experimental studies provide evidence for both of the two seemingly contradictory views that either complex transient dynamics implement WM (e.g. Jun et al., 2010; Hussar and Pasternak, 2012) or that persistent activity as resulting from attractor dominated dynamics suffices to explain WM operation (e.g. Zhou et al., 2013; Wimmer et al., 2014). Here, we verify the first sub-hypothesis of this thesis (Figure 1.1) according to which the neuronal systems implementing WM actually require both, transient neuronal dynamics and distinct attractor states, to enable robust memory storage and complex computations.

The n-back task is an established measure of WM performance. We show that a purely transient network model is able to solve this task with predictable temporal structure by storing the information about the past stimuli in distinguishable trajectories. However, if we introduce uncertainty in the timing of stimuli, this storage mechanism is disturbed as the trajectory bundles representing different stimulus histories overlap. This overlap impedes the readout of the desired target signal. In contrast, human subjects do not show reduced performance in the n-back task with unpredictable stimulus timing (Koppe et al., 2014). We show that introducing distinct attractor states into the network dynamics, which store the relevant information about the past stimuli, leads to a structuring of the phase space of the system. Now, the trajectory bundles which correspond to the different stimulus histories remain 
well separated also in the case of unpredictable stimulus timing. The corresponding phase of transient dynamics triggered by an incoming stimulus is both necessary and sufficient to produce a complex time-dependent signal at the readout unit, i.e. to perform complex temporal computations. Thus, only a network exploiting distinct attractor states as well as transient dynamics is able to solve the n-back task with unpredictable stimulus timing. This verifies our first sub-hypothesis of this thesis.

We use the well studied class of reservoir networks as a model of neuronal systems which operate on purely transient dynamics (Jaeger, 2001; Maass et al., 2002; Lukoševičius et al., 2012). The robustness of reservoir networks with respect to additional or multiplicative noise in the input signal has been extensively studied (Maass and Sontag, 1999; Rabinovich et al., 2006; Lukoševičius and Jaeger, 2009; Laje and Buonomano, 2013). The vulnerability of reservoir networks with respect to noise in the temporal structure of a task, in contrast, has not been analyzed before. The dynamical origin of the inability to solve a task with unpredictable temporal structure is due to the very general principle of storing information about the recent stimulus history in distinct trajectories. We therefore expect that this inability can be generalized to other types of neuronal networks which store information in a purely transient manner (for a review, see Buonomano and Maass, 2009). However, a detailed investigation of the performance of other transient storage mechanisms in tasks with unpredictable temporal structure remains to be done.

Reservoir networks with additional attractor dynamics have been discussed in a general context (Sussillo and Barak, 2013) and in the context of WM (Jaeger and Eck, 2008; Pascanu and Jaeger, 2011) but not specifically in the context of solving task with unpredictable temporal structure. As in this chapter, also these studies introduce the attractor states via feedback loops through additional readout neurons. These feedback signals do not only enhance the stability and the performance of the network but can also enable universal computational power (Maass et al., 2007; Sussillo and Abbott, 2009; Gros, 2009). Actually, the activities in a reservoir network representing context signals via feedback from explicitly trained readout neurons resembles the neuronal activity in monkey cortex (Enel et al., 2016). Instead of training explicit additional readout units, also the internal synaptic weights within the generator network can be adapted to obtain equivalent attractor states (Sussillo and Abbott, 2012). Note that the states used to stably store the relevant information possibly do not necessarily need to be real attractors of the systems. Instead, it might also suffice to employ slow-states of the dynamics (Sussillo and Barak, 2013) or attractor relics as exploited in saddle point networks and heteroclinic networks (Rabinovich et al., 2008b; Gros, 2009; Bick and Rabinovich, 2009). As long as the dynamics of these states evolve on a slower time scale than the transient neuronal dynamics, they might structure the phase space of the neuronal activity in a similar manner as discussed above. Additionally, these attractor or attractor-like states do not necessarily have to be realized by persistent neuronal activities. Instead, they might also be implemented by short-term synaptic dynamics (Mongillo et al., 2008; Rose et al., 2016a).

Several experimental results agree with our finding that a combination of both transient dynamics and attractor states underly the dynamics of WM. In the monkey PFC, incoming stimuli during a WM task trigger neuronal dynamics which can be described as a fast transient transition through the activity space before ending up in a low activity attractor state (Stokes et al., 2013). Imaging studies of the human PFC reveal similar dynamics (Courtney et al., 1997). Furthermore, recent experimental results confirm the important role of persistent activity in the PFC in encoding information during different WM tasks (Riley and Constantinidis, 2016). An interplay of transient dynamics and attractor states is also found in other contexts in the brain, 
for instance, in the odor system of the locust (Mazor and Laurent, 2005).

In this chapter, we demonstrate the necessity of attractor states for the neuronal systems implementing WM as stated by our first sub-hypothesis. We introduce these attractor states by the addition of two appropriately trained additional readout units whose activities represent the relevant information about past stimuli (Section 3.2.3). This raises the question how such meaningful attractor states may emerge in the brain. According to the main hypothesis of this thesis, the attractor states are not formed in the WM system itself. Instead, they emerge in a self-organized way in the interacting memory systems. The attractor states which are required for robust memory storage in WM need to represent the types of the observed stimuli. For instance, in the WM task studied by Koppe et al., (2014), they need to represent triangles, squares and combinations of these. If these types are known to the subject performing the WM task, they are represented in the declarative parts of LTM. As discussed in Section 2.3, information in LTM is generally assumed to be encoded in distinct attractor states (Wood et al., 2011). Thus, by ways of mutual interactions, the attractor states formed in a self-organized way in the LTM system might be the basis of robust storage of information in WM. To evaluate this possibility, we need to understand the mechanisms which allow the self-organized formation of attractor states and their correct assignment to different stimuli in the neuronal system implementing LTM. These are covered by the second sub-hypothesis of this thesis which is the subject of the following chapter. 


\section{Chapter 4}

\section{Self-Organized Formation and Allocation of Cell Assemblies}

In the previous chapter, we show that the neuronal system implementing working memory (WM) requires attractor states (or attractor-like states, see Section 3.3) for solving WM tasks with unpredictable temporal structure. This yields the question how such attractor states, which represent the current history of stimuli, may emerge in the brain. Following the main hypothesis of this thesis, we propose that these attractor states are not formed in the specific neuronal system implementing WM but rather in different interacting memory systems. In particular, the declarative part of the long-term memory (LTM) is assumed to be implemented by input dependent attractor states which represent facts, events and concepts (Section 2.3). These concepts may be of abstract nature and may represent, for instance, the different types of stimuli experienced during a WM task. Thus, in order to understand the self-organized emergence of the attractor states which allow the stable information storage in WM, we need to understand the mechanisms which underly the formation and allocation of attractor states in LTM.

According to the Hebbian postulates (Section 2.2), a previously unknown stimulus leads to the activation of a certain group of neurons in the neuronal system implementing LTM. As a result, the weights of the synapses which interconnect these active neurons are potentiated by activity-dependent synaptic plasticity or, more specific, Hebbian plasticity. This leads to the formation of a group of strongly interconnected neurons called a cell assembly (CA). This CA is the new mental representation of the previously unknown stimulus. A later recall of this stimulus corresponds to the reactivation of the respective $\mathrm{CA}$. In other terms, recognizing two slightly different stimuli as being of the same type requires both stimuli to activate the same CA. This is possible if two key conditions are met. On the one hand, as described, the formation of a CA leads to strong recurrent synapses in between the respective neurons. This is required to enable pattern completion, i.e., the activation of the complete CA from just a fraction of the original stimulus (Palm et al., 2014; Hunsaker and Kesner, 2013). On the other hand, the feedforward synapses which project the different stimuli to the different CAs need to be accurately adjusted to ensure that only specific stimuli are able to evoke activity within a given CA. This process is referred to as memory assignment or memory allocation. Most existing studies, both theoretical and experimental ones, may either explain the mechanisms involved in the formation of CAs (Tetzlaff et al., 2013; Litwin-Kumar and Doiron, 2014; Zenke et al., 2015; Holtmaat and Caroni, 2016) or the processes involved in the allocation of specific stimuli to respective neuronal representations (Kohonen, 1982; Sullivan and Sa, 2006; Stevens et al., 2013; Rogerson et al., 2014). It is still unknown, however, how the neuronal system is able to reliably coordinate both processes simultaneously in a self-organized way. 
In this chapter, we show that attractor states representing different stimuli can be formed and assigned reliably in a self-organized way by two interacting forms of synaptic plasticity, Hebbian plasticity and synaptic scaling, in the LTM system. This corresponds to the verification of the second sub-hypothesis of this thesis (Figure 1.1). We present a neuronal network model in which stimuli are projected via feedforward synapses onto a recurrent area containing neurons which interact via recurrent synapses (Section 4.1). Both the feedforward and the recurrent synapses are subject to a combination of Hebbian plasticity and synaptic scaling (compare Section 2.1). Based on different topological and functional measures, we study the self-organized formation and allocation of CAs in this network model (Section 4.2). We derive a reduced population model of the network dynamics which allows for an analytical treatment. This enables the identification of three generic properties of the synaptic plasticity rule which allow the reliable formation and allocation of CAs (Section 4.3). Finally, we summarize and discuss the obtained results and its implications within the context of this thesis (Section 4.4).

Many of the results presented here are taken from a submitted manuscript:

J. M. Auth, T. Nachstedt, and C. Tetzlaff (2017). The interplay of synaptic plasticity and scaling enables the self-organized allocation of multiple memory representations. Submitted.

\subsection{Methods}

In this section, first, we present in detail the actual network model used to study the self-organization of CAs. We continue by defining the topological and functional measures used to investigate the CAs which emerge in this network and their dynamic behavior. Finally, we give a brief overview of the most relevant concepts employed in the analytical investigations of the population model in Section 4.3.

\subsubsection{Network Model}

The model which we study in this chapter is based on the recurrent network model presented by Tetzlaff et al., (2013). We extend this model by a set of plastic feedforward synapses. These synapses transmit the signals received from a lower level brain area into the recurrent area. In addition, we replace the local inhibition in this recurrent area by a global inhibitory mechanism.

\section{Network Topology}

The network model consists of three distinct neuronal areas (Figure $4.1 \mathrm{a}$ ): an input area, a recurrent area and an inhibitory population.

Signals or stimuli arriving from other brain areas are represented by the input area. In the input area, a stimulus is encoded by activating a subset of the $N^{\text {in }}$ excitatory neurons. Feedforward synapses project the firing rates of these neuron into the recurrent area. The recurrent area consists of $N^{\text {rec }}$ recurrently connected excitatory neurons. These neurons are distributed on a grid and interact via synapses with a distance dependent-topology. We apply periodic boundary conditions to avoid boundary effects. Every neuron in the recurrent area receives feedforward synaptic signals from exactly $n^{\mathrm{ff}}$ neurons in the input area. The corresponding synaptic weights are denoted as $w_{i k}^{\mathrm{ff}}$ with $i \in\left\{1, \ldots, N^{\mathrm{rec}}\right\}$ and $k \in\left\{1, \ldots, N^{\mathrm{in}}\right\}$. Additionally, every neuron in the recurrent area receives excitatory synaptic signals from all other 


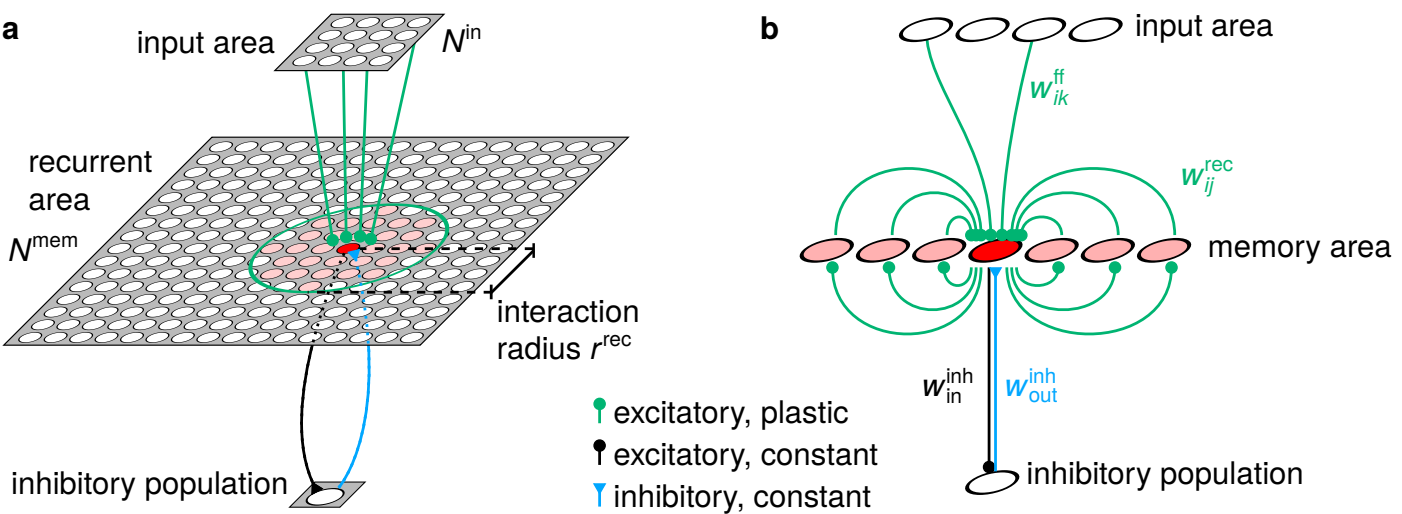

Figure 4.1: Network model for studying the self-organized formation of CAs. (a) The network is divided into three distinct areas. The neurons in the input area are externally controlled and represent the different stimuli presented to the network. Neurons in the input area project signals through feedforward synapses into the recurrent area composed of excitatory neurons distributed on a grid. The interaction radius $r^{\text {rec }}$ determines the distance-dependent synaptic topology of the neurons within the recurrent layer (green circle). A neuron receives signals from all other neurons in the recurrent area whose euclidean distance measured in grid units is smaller than $r^{\text {rec }}$. All neurons in the recurrent area excite the inhibitory population which, in turn, transmits inhibitory signals to all neurons in the recurrent area. In the figure, only the synaptic connections of one neuron (red neuron) in the recurrent area are shown as an example. (b) The synapses transmitting signals from the input area to the recurrent area and the recurrent synapses in the recurrent area are subject to synaptic plasticity. The outgoing and ingoing synapses at the inhibitory population are static.

neurons in the recurrent area whose distance on the grid is smaller than the recurrent interaction radius $r^{\text {rec }}$. The respective synaptic weights are referred to as $w_{i j}^{\text {rec }}$ with $i, j \in\left\{1, \ldots, N^{\text {rec }}\right\}$. Every neuron in the recurrent area also receives inhibitory signals through synapses with weights $w_{\text {out }}^{\text {inh }}$ from the inhibitory population. The inhibitory population, in turn, receives synaptic connections with weight $w_{\text {in }}^{\text {inh }}$ from all neurons in the recurrent area.

All synaptic connections in between excitatory neurons are subject to synaptic plasticity (Figure $4.1 \mathrm{~b}$ ). Only the synapses transmitting signals into and from the inhibitory population are assumed to be static.

\section{Model Dynamics}

The models used to describe the neurons and plastic synapses in the network are chosen according to the models used by Tetzlaff et al., (2013). All neurons in the network are described by a rate-coded neuron model. The synapses in between excitatory neurons are governed by a combination of Hebbian plasticity and synaptic scaling. In contrast to Tetzlaff et al., (2013), we normalize both the neuronal dynamics and the synaptic dynamics to reduce the number of covariant parameters. In the normalized formulation, the firing rate of a neuron ranges between zero and one. Similarly, the equilibrium weight of a synapse in between two neurons with maximum firing rate is also one. The details about how to convert the original model into the normalized version are given in Appendix A.1.

Every neuron in the recurrent area is described by a leaky membrane potential $u_{i}$, $i \in\left\{1, \ldots, N^{\text {rec }}\right\}$, which develops according to

$$
\tau \frac{\mathrm{d} u_{i}}{\mathrm{~d} t}=-u_{i}+\sum_{j \in C_{i}^{\text {rec }}} w_{i j}^{\mathrm{rec}} F_{j}+\sum_{k \in C_{i}^{\mathrm{ff}}} w_{i k}^{\mathrm{ff}} I_{k}+w_{\mathrm{out}}^{\mathrm{inh}} F^{\mathrm{inh}} .
$$


Here, the time constant $\tau$ characterizes the time scale of the dynamics. The set $C_{i}^{\text {rec }}$ contains the indices $j$ of neurons within the recurrent area which transmit synaptic signals to the neuron with index $i$. Accordingly, $C_{i}^{\mathrm{ff}}$ is the set of the indices $k$ of all neurons in the input area which project signals to the neuron $i$ within the recurrent area. The activity level of the neuron with index $k$ in the input area is given by $I_{k}$. The weight $w_{\text {out }}^{\text {inh }}$ determines the uniform coupling of the inhibitory population to the neurons within the recurrent area.

The firing rate $F_{i}$ of neuron $i$ within the recurrent area is given by a sigmoidal firing rate function $\phi$ of the membrane potential $u_{i}$ :

$$
F_{i}=\phi\left(u_{i}\right)=\frac{1}{1+\exp \left(\beta\left(\epsilon-u_{i}\right)\right)} .
$$

The parameter $\epsilon$ determines the location of the inflection point, i.e., of the steepest point of the firing rate function. The parameter $\beta$ controls the overall steepness of the function.

The inhibitory population is modeled like a single neuron. The dynamics of its membrane potential $u^{\text {inh }}$ is given by

$$
\tau^{\mathrm{inh}} \frac{\mathrm{d} u^{\mathrm{inh}}}{\mathrm{d} t}=-u^{\mathrm{inh}}+\sum_{i}^{N^{\text {rec }}} w_{\text {in }}^{\text {inh }} F_{i}
$$

where the time constant $\tau^{\text {inh }}$ defines the time scale of the dynamics of the inhibitory population. The synaptic weight $w_{\text {in }}^{\text {inh }}$ specifies the strengths of the synapses transmitting the activity of the excitatory neurons to the inhibitory population. Analogously to the firing rate of the excitatory neurons, the firing rate $F^{\text {inh }}$ of the inhibitory population is determined by a sigmoidal firing rate function $\phi^{\text {inh }}$ with parameters $\beta^{\text {inh }}$ and $\epsilon^{\text {inh: }}$

$$
F^{\text {inh }}=\phi^{\text {inh }}\left(u^{\text {inh }}\right)=\frac{1}{1+\exp \left(\beta^{\text {inh }}\left(\epsilon^{\text {inh }}-u^{\text {inh }}\right)\right)} .
$$

The dynamics of the recurrent synapses in between the excitatory neurons in the recurrent area (compare Figure $4.1 \mathrm{~b}$ ) is given by a normalized version of the combination of Hebbian plasticity and homeostatic synaptic scaling introduced by Tetzlaff et al., (2011). The weight $w_{i j}^{\text {rec }}$ of the synapse transmitting signals from neuron $j$ to neuron $i$ follows the following dynamics:

$$
\tau^{\mathrm{rec}} \frac{\mathrm{d} w_{i j}^{\mathrm{rec}}}{\mathrm{d} t}=F_{i} F_{j}+\frac{F^{\mathrm{T}}-F_{i}}{1-F^{\mathrm{T}}}\left(w_{i j}^{\mathrm{rec}}\right)^{2} .
$$

In this normalized plasticity model, the time constant $\tau^{\text {rec }}$ and the target firing rate $F^{\mathrm{T}}$ of the homeostatic process are the only free parameters.

The dynamics of the feedforward synapses follows the same plasticity rule. The weight $w_{i k}^{\mathrm{ff}}$ of the feedforward synapse transmitting signals from neuron $k$ in the input area to neuron $i$ in the recurrent area follows the following dynamics:

$$
\tau^{\mathrm{ff}} \frac{\mathrm{d} w_{i k}^{\mathrm{ff}}}{\mathrm{d} t}=F_{i} I_{k}+\frac{F^{\mathrm{T}}-F_{i}}{1-F^{\mathrm{T}}}\left(w_{i k}^{\mathrm{ff}}\right)^{2}
$$

with a time constant $\tau^{\mathrm{ff}}$ determining the timescale of the plasticity process. 


\section{Parameters}

The described network model contains a number of topological and dynamical parameters. In the following, we discuss the used set of parameter values.

Concerning the topological parameters of the model, the number of neurons in the recurrent area is chosen to be $N^{\text {rec }}=30 \times 30=900$. On the one hand, this number is large enough to allow the application of tools tailored for the analysis of large dynamical systems, for instance mean-field approaches. On the other hand, it ensures computational tractability when simulating the network. The interaction radius within the recurrent area is $r^{\mathrm{rec}}=3$. Consequently, every neuron in the recurrent area receives synaptic signals from $n^{\text {rec }}=28$ other neurons in this area. In addition, every neuron in the recurrent area receives synaptic signals from $n^{\mathrm{ff}}=25$ independently chosen neurons in the input area. The number of neurons in the input area is $N^{\text {in }}=100$.

The time scales of the excitatory and inhibitory neurons are typically in the range of tens of milliseconds (Tetzlaff et al., 2012a). Thus, we use $\tau=\tau_{\text {inh }}=10 \mathrm{~ms}$. The time scale of long-term synaptic plasticity, on the other hand, is several orders of magnitudes slower (Tetzlaff et al., 2012a). We choose $\tau^{\text {rec }}=\tau^{\mathrm{ff}}=10 \mathrm{~s}$. On the one hand, this significantly larger than the neuronal time scales. On the other hand, it still allows reasonable simulation times.

The target firing rate $F^{\mathrm{T}}$ of the synaptic scaling term in Equations (4.5) and (4.6) is a further free parameter of the synaptic plasticity model. In previous studies either very small values (Tetzlaff et al., 2012b) or a zero value (Tetzlaff et al., 2013) have been used for $F^{\mathrm{T}}$. Here, we consider the fact that the Hebbian terms in Equations (4.5) and (4.6) only describe long-term potentiation and no long-term depression of synapses. Therefore, the synaptic scaling terms only need to balance the potentiation of the synaptic weights. If $F^{\mathrm{T}}$ is chosen to be a strictly positive value, both Hebbian plasticity and synaptic scaling yield to potentiation for postsynaptic firing rates lower than $F^{\mathrm{T}}$. Hence, no equilibrium exists in this regime. This may result in diverging weights, for instance, if the postsynaptic neuron receives strong inhibitory inputs. We choose $F^{\mathrm{T}}=0$ to avoid these kind of instabilities.

The parameters of the firing rate function of the excitatory neurons determine how the neuron reacts to presynaptic inputs. For the position of the inflection point, we choose $\epsilon=12$. As a result, 12 fully active presynaptic neurons may drive a postsynaptic neuron to half of its maximum firing rate. Furthermore, we use $\beta=1$ as the overall steepness of the function. This means that few more than 12 fully active inputs suffice to drive the neuron close toward its maximum firing rate.

The strength of the synapses transmitting signals from neurons in the recurrent area to the inhibitory population is $w_{\text {in }}^{\text {inh }}=1$. This parameter is covariant with the parameters of the firing rate function of the inhibitory population. For this firing rate function, we choose $\epsilon^{\text {inh }}=100$ and $\beta^{\text {inh }}=1$. The position $\epsilon^{\text {inh }}$ of the inflection point is chosen such that it starts to balance the excitatory activity if approximately 100 neurons in the recurrent area are active. The strength of the synapses transmitting signals from the inhibitory population to the recurrent area is $w_{\mathrm{out}}^{\mathrm{inh}}=-20$. This strength needs to be large enough to balance the sum of excitatory signals which arrive at a neuron in the recurrent area.

When numerically solving the network dynamics, the initial weights of the recurrent and feedforward synapses are $w_{i j}^{\text {rec }}(t=0)=w_{i k}^{\mathrm{ff}}(t=0)=0.5$ for all values of $i, j$ and $k$ that belong to existing synapses. Table 4.1 summarizes all used parameters and their respective values. If not stated differently, this set of parameter values is used in all simulations whose results we present in the following. 
Table 4.1: Overview of the network model parameters and their values

\begin{tabular}{llr}
\hline Parameter Description & Symbol & Value \\
\hline number of neurons in the recurrent area & $N^{\text {rec }}$ & 900 \\
number of neurons in the input area & $N^{\text {in }}$ & 100 \\
excitatory interaction radius & $r^{\text {rec }}$ & 3 \\
number of feedforward synapses per neuron in the recurrent area & $n^{\text {ff }}$ & 25 \\
time constant of excitatory neurons & $\tau$ & $10 \mathrm{~ms}$ \\
time constant of inhibitory population & $\tau^{\text {inh }}$ & $10 \mathrm{~ms}$ \\
weight of synapses from inhibitory population to recurrent area & $W_{\text {out }}^{\text {inh }}$ & -20 \\
weight of synapses from recurrent area to inhibitory population & $W_{\text {in }}^{\text {inh }}$ & 1 \\
steepness of excitatory firing rate function & $\beta$ & 1 \\
inflection point of excitatory firing rate function & $\epsilon$ & 12 \\
steepness of inhibitory firing rate function & $\beta^{\text {inh }}$ & 1 \\
inflection point of inhibitory firing rate function & $\epsilon^{\text {inh }}$ & 100 \\
time constant of recurrent synaptic plasticity & $\tau^{\text {rec }}$ & $10 \mathrm{~s}$ \\
time constant of feedforward synaptic plasticity & $\tau^{\mathrm{ff}}$ & $10 \mathrm{~s}$ \\
target firing rate of synaptic scaling & $F^{\mathrm{T}}$ & 0 \\
initial weight of recurrent synapses & $W_{i j}^{\text {rec }}(t=0)$ & 0.5 \\
initial weight of feedforward synapses & $W_{i k}^{\mathrm{ft}}(t=0)$ & 0.5 \\
\hline
\end{tabular}

\section{Numerical Integration}

For numerically solving the network dynamics, we integrate the network model using the Euler method (Butcher, 2003) with a time step of $\Delta t_{\text {euler }}=1 \mathrm{~ms}$.

\subsubsection{Topological and Functional Measures}

When analyzing the described network model, we use different topological and functional measures to capture the characteristic properties of the representations emerging in the recurrent area. These are defined and motivated in the following.

\section{Active Neurons}

We refer to neurons whose current firing rate $F_{i}$ is higher than 0.5 as active neurons. Neurons with a firing rate $F_{i}<0.5$ are called inactive. The number of currently active neurons in the recurrent area is denoted as $N_{+}^{\text {rec }}$. When we present a stimulus to the network, we set the firing rate of every neuron in the input area to either zero (inactive) or one (active). The number of currently active neurons in the input area is denoted as $N_{+}^{\text {in }}$. We call the number of incoming recurrent synapses at neuron $i$ that transmit signals from active neurons in the recurrent area $n_{i,+}^{\text {rec }}$. The quantity $n_{i,+}^{\mathrm{ff}}$ gives the number of feedforward synapses which originate from active input neurons and converge at neuron $i$ in the recurrent area.

\section{Active Neighbor Ratio}

A CA is defined as a group of highly interconnected neurons (compare Section 2.2). If the set of currently active neurons in the recurrent area is a CA, every active neuron receives synaptic signals from many other active neurons in the recurrent area. This means in case of a CA, the active neurons form a cluster in the topology defined by the synaptic connectivity. To evaluate the degree of clustering, we introduce a measure which captures the ratio of the number $n_{i,+}^{\text {rec }}$ of incoming recurrent synapses from active neurons and the total number $n^{\text {rec }}$ of incoming recurrent synapses. We 
a unclustered activity

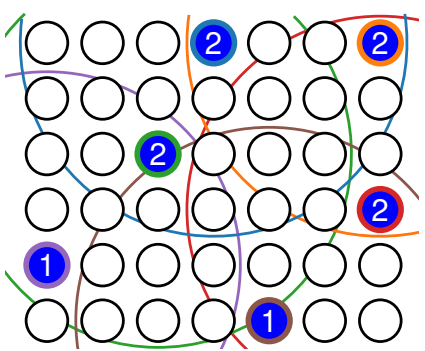

b

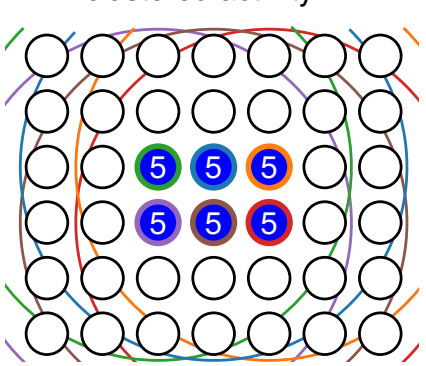

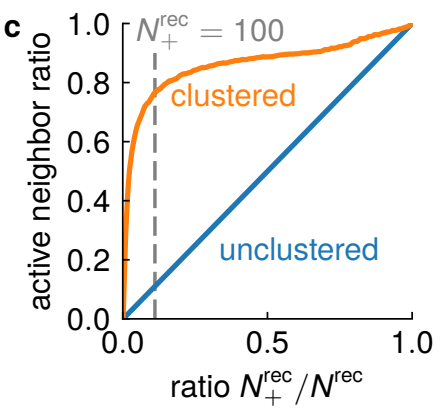

Figure 4.2: Active neighbor ratio (ANR) as a measure of activity clustering. The ANR is given by the mean ratio of the number of recurrent synapses originating from active $\left(F_{i}>0.5\right)$ neurons and the number of all incoming recurrent synapses for active neurons. (a) In the case of unclustered activity, the proportion of synapses transmitting signals from active neurons in the recurrent area is low. Here, active neurons are filled blue. The circles in the background indicate the recurrent interaction radius $r^{\text {rec }}$ of these neurons. For each active neuron, the number of other active neurons within this circular neighborhood is given. Note that only these values influence the ANR. (b) If the same number of active neurons is clustered at a specific location in the network, the number of synapses transmitting signals from active neurons in the recurrent area is much higher in these neurons. (c) Expected ANR for unclustered activity and for perfectly clustered activity in the recurrent area of the network as a function of the number $N_{+}^{\text {rec }}$ of active neurons in this area. The dashed vertical line indicates the value $N_{+}^{\text {rec }}=100$ as this approximate value is of relevance later on.

define the active neighbor ratio ANR of a network state as the mean of this ratio over all currently active neurons in the recurrent area:

$$
\mathrm{ANR}=\frac{1}{N_{+}} \sum_{\substack{i=1,1 \\ F_{i}>0.5}}^{\mathrm{N}^{\mathrm{rec}}} \frac{n_{i,+}^{\mathrm{rec}}}{n^{\mathrm{rec}}}
$$

If the currently active neurons are not clustered, i.e., randomly located in the recurrent area (Figure 4.2 a), the ANR depends linearly on the number $N_{+}$of active neurons (Figure $4.2 \mathrm{c}$ ). The expected value of the ANR for a perfect clustering of the neuronal activity (Figure $4.2 \mathrm{~b}$ ), in contrast, is much higher (Figure $4.2 \mathrm{c}$ ). The actual ANR difference between the clustered and the unclustered case depends on the number $N_{+}$of active neurons in the recurrent area. If $N_{+}$is close to $N^{\text {rec }}$, almost all neurons in the recurrent area are active. Thus, the ANR difference between clustered and unclustered activity is small. This is a finite size effect.

\section{Pattern Completion and Separation}

Pattern completion and pattern separation are fundamental capabilities of memory systems (Bakker et al., 2008; Hunsaker and Kesner, 2013). In very general terms, a memory system maps different input stimuli to corresponding memory representations. If the difference between two representations is smaller than the difference between the two respective input stimuli, this process is called pattern completion. If the difference between the representations is higher than the difference between the stimuli, this is referred to as pattern separation.

To quantify whether a system performs pattern completion or pattern separation for a given pair of input stimuli A and B, we introduce a measure for the similarity between the input stimuli and between the memory representations. We define the set $S_{\mathrm{A}}$ of neurons in the input area which are active when stimulus $\mathrm{A}$ is presented to the network. The corresponding memory representation $R_{\mathrm{A}}$ of stimulus $\mathrm{A}$ is given 
by the set of neurons in the recurrent area which are active when $\mathrm{A}$ is presented to the network. Accordingly, the set $S_{\mathrm{B}}$ contains the neurons in the input area which are active when stimulus $B$ is presented to the network. The corresponding active neurons in the recurrent area define the set $R_{\mathrm{B}}$.

We use the Jaccard index $J$ as a measure of the overlap between the two input stimuli and between the two memory representations. Given two sets, the Jaccard index $J$ is defined as the ratio of the size of the intersection and the size of the union of these two sets. In consequence, $J$ is zero for two disjunct sets and one for two identical sets. Given the sets $S_{\mathrm{A}}, S_{\mathrm{B}}, R_{\mathrm{A}}$, and $R_{\mathrm{B}}$, we have

$$
J\left(S_{\mathrm{A}}, S_{\mathrm{B}}\right)=\frac{\left|S_{\mathrm{A}} \cap S_{\mathrm{B}}\right|}{\left|S_{\mathrm{A}} \cup S_{\mathrm{B}}\right|} \text { and } J\left(R_{\mathrm{A}}, R_{\mathrm{B}}\right)=\frac{\left|R_{\mathrm{A}} \cap R_{\mathrm{B}}\right|}{\left|R_{\mathrm{A}} \cup R_{\mathrm{B}}\right|} .
$$

If the Jaccard index $J\left(R_{\mathrm{A}}, R_{\mathrm{B}}\right)$ of the two memory representations is larger than the Jaccard index $J\left(S_{\mathrm{A}}, S_{\mathrm{B}}\right)$ of the two input stimuli $\left(J\left(R_{\mathrm{A}}, R_{\mathrm{B}}\right)>J\left(S_{\mathrm{A}}, S_{\mathrm{B}}\right)\right)$, pattern completion has occurred. In contrast, if the Jaccard index of the representations is lower than the Jaccard index of the input stimuli $\left(J\left(R_{\mathrm{A}}, R_{\mathrm{B}}\right)<J\left(S_{\mathrm{A}}, S_{\mathrm{B}}\right)\right)$, the network performs pattern separation for these stimuli.

\section{Network and Ensemble Averages}

In the following, we use different kinds of mean values to discuss the properties of individual neurons or synapses. We denote a mean value of a property $x$ over a certain group of neurons or synapses within a single network instantiation as $\bar{x}$. The average of this mean value over an ensemble of several independent network instantiations is denoted as $\langle\bar{x}\rangle$.

\subsubsection{Nullclines, Equilibria and Asymptotic Stability}

In Section 4.3, we reduce the network dynamics to a low-dimensional model. We analyze this model using methods of standard stability theory. Here, we briefly summarize the most relevant concepts for this analysis.

We assume $\dot{x}=\frac{\mathrm{d} x}{\mathrm{~d} t} x=f(x)$ to be an autonomous system of $n$ ordinary differential equations:

$$
\begin{gathered}
\dot{x}_{1}=f_{1}\left(x_{1}, \ldots, x_{n}\right) \\
\dot{x}_{2}=f_{2}\left(x_{1}, \ldots, x_{n}\right) \\
\quad \vdots \\
\dot{x}_{n}=f_{n}\left(x_{1}, \ldots, x_{n}\right) .
\end{gathered}
$$

When we set each of the $n$ differential equations equal to zero $\left(\dot{x}_{i}=0\right.$ for $i \in$ $\{1, \ldots, n\})$, we obtain the $n$ nullclines of the system. The $i^{\prime}$ th nullcline is defined as the geometric shape in the phase space for which $\dot{x}_{i}=0$ holds. A point $x^{*}:=$ $\left(x_{1}^{*}, x_{2}^{*}, \ldots, x_{n}^{*}\right)$ in the phase space in which all the $n$ nullclines intersect is an equilibrium of the system. For this point, we have $f\left(x^{*}\right)=0$. Alternatively, the term fixed point is used interchangeably with the term equilibrium.

The dynamics of the system in the surroundings of an equilibrium $x^{*}$ determines the asymptotic stability of this equilibrium. If there is a region around $x^{*}$ from within which the system converges back to $x^{*}$, the equilibrium is called asymptotically stable or attracting. If no such region exist and even infinitesimally small perturbations suffice to drive the system away from the equilibrium, it is unstable or repulsive. Whether a given equilibrium $x^{*}$ of a system is asymptotically stable can be determined 
by evaluating the eigenvalues of the Jacobian matrix $D_{x} f$ of the system at $x^{*}$. If the real parts of all eigenvalues are negative, the given equilibrium is asymptotically stable. If the real part of at least one eigenvalue is larger than zero, the equilibrium is an unstable one. If the eigenvalue with the largest real part lies on the imaginary axis, linear stability theory is not conclusive about its stability and higher order terms have to be considered (Kuznetsov, 1998).

\subsection{Cell Assembly Dynamics}

In the following, we study the self-organized formation of CAs in the described network model. We start with an overview of the general process of CA formation and assignment in the model. Afterwards, we investigate different topological and functional properties of the formed CAs.

\subsubsection{General Cell Assembly Formation}

We initiate the process of self-organized CA formation by presenting a stimulus pattern in the input area, i.e., by activating a subset of the neurons in the input area (Figure $4.3 \mathrm{a}$, top row). This activity is transmitted to neurons in the recurrent area by the feedforward synapses. In the example discussed here, the resulting signals arriving at neurons in the recurrent area initially do not suffice to elicit significant firing rates $(t=0 \mathrm{~s})$. This may be different for a larger number $N_{+}^{\text {in }}$ of activated neurons in the input area. Here, however, the onset of the stimulus is first followed by a phase of very low activity in the recurrent area. Nevertheless, due to the large presynaptic activity, the weights of the feedforward synapses transmitting signals from the active neurons in the input area are slowly potentiated.

As a result of this potentiation, at some point of time, the membrane potential of neurons in the recurrent area which receive synaptic signals from a high number of active neurons in the input area reaches the inflection point of the firing rate function. These neurons do now show significant firing activity $(t=20 \mathrm{~s})$. This leads to the potentiation of the recurrent synapses in between active neurons in the recurrent area. Additionally, due to the high presynaptic activity, also the weights of synapses from active neurons in the recurrent area to inactive neighboring neurons are increased. This increases the probability of these neighboring neurons to also receive enough excitatory inputs to get active. At some point, this ongoing process of self-organization leads to a cluster of strongly interconnected active neurons or, in other words, a CA $(t=40 \mathrm{~s})$. This is also reflected by the time course of the ANR in the network which initially takes low values and then increases to an intermediate value (Figure $4.3 \mathrm{~d}$ ). This indicates a transition from a state of unclustered activity in the recurrent area to a state in which a CA coexists with some active neurons which do not possess a high number of synaptic connections with other active neurons.

The ongoing plasticity at both the recurrent and the feedforward synapses increases the number of neurons involved in the CA (Figure $4.3 \mathrm{c}$ ). The plasticity of the feedforward synapses potentiates the synapses projecting signals from active neurons in the input are to neurons in the CA. In parallel, synaptic scaling decreases the weights of synapses which connect inactive neurons in the input area to active neurons in the recurrent area (bottom row in Figure $4.3 \mathrm{a}$ ). The downscaling is important for the separation of distinct patterns as will be discussed in Section 4.3.3.

The recruitment of more and more neurons into the CA results in stronger activation of the inhibitory population. This leads to stronger inhibitory signals received 


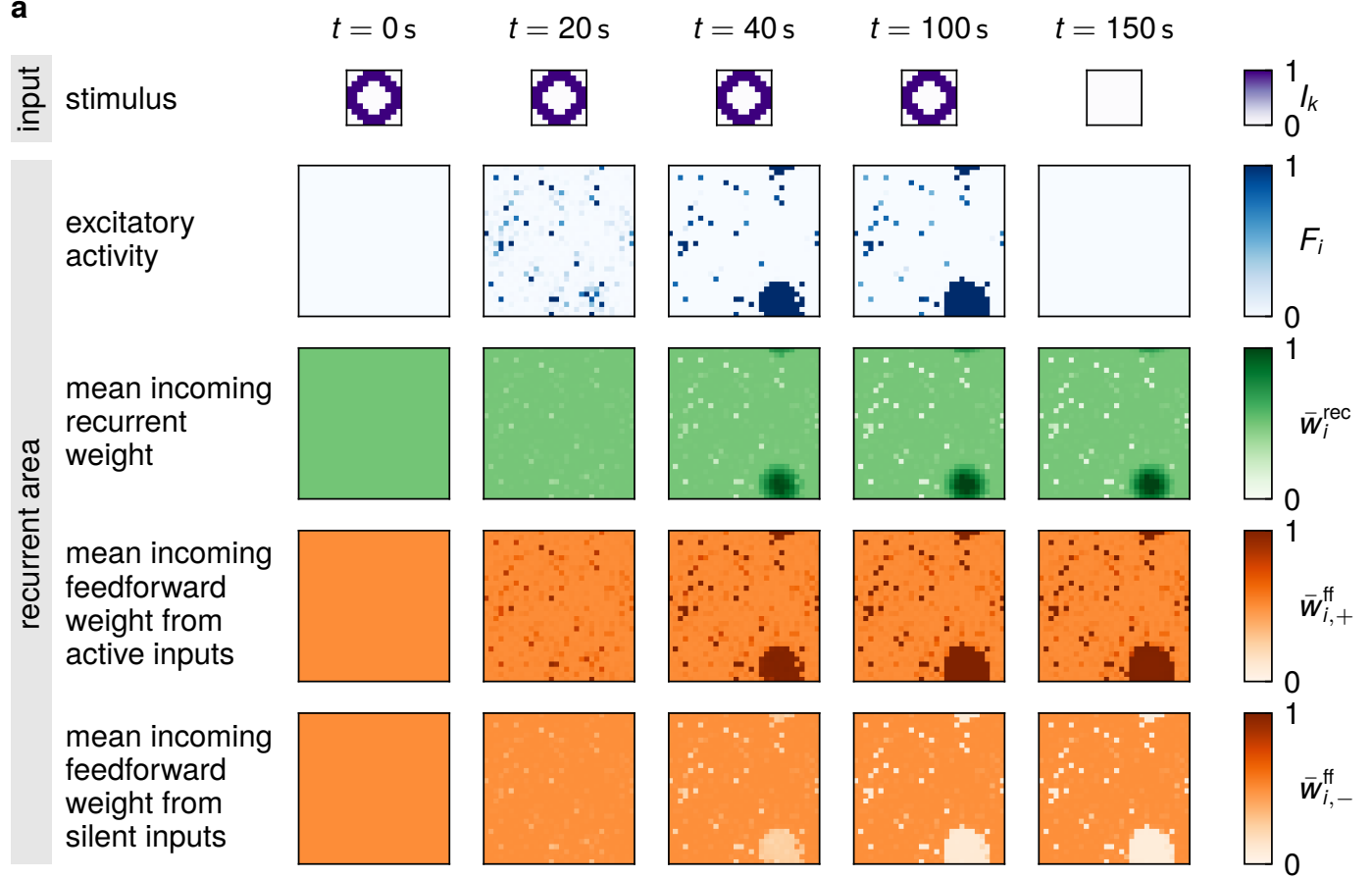

b

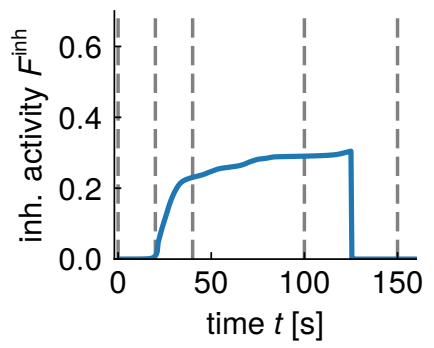

C

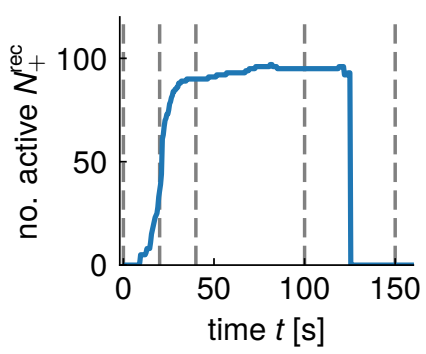

d

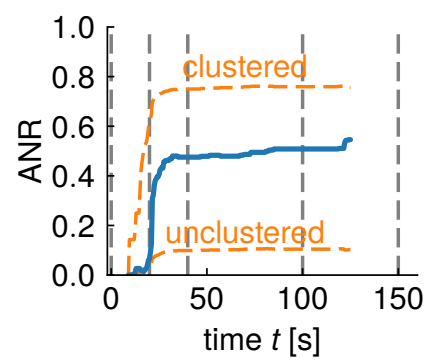

Figure 4.3: Self-organized formation of a CA. (a) Snapshots of the activities $I_{k}$ of the neurons within the input area, the excitatory activity $F_{i}$ of neurons within the recurrent area, the mean weights $\bar{w}_{i}^{\text {rec }}$ of the incoming recurrent synapses per neuron, and the mean weights $\bar{w}_{i,+}^{\mathrm{f}}$ and $\bar{w}_{i,-}^{\mathrm{Hf}}$ of incoming feedforward synapses originating from active or inactive input neurons, respectively, at different point of times during the CA formation. The input stimulus is active for $0 s \leq t \leq 125 \mathrm{~s}$. (b) The activity $F^{\text {inh }}$ of the inhibitory population increases rapidly during the initial formation of the CA. Afterwards it increases slowly. Note that $F^{\text {inh }}$ depends monotonously on the total excitatory activity in the recurrent area. (c) The number $N_{+}^{\text {rec }}$ of active neurons, i.e., neurons with a firing rate $F_{i}>0.5$, increases only slightly once the CA has emerged $(t>50 \mathrm{~s})$. (d) The active neighbor ratio (ANR) in the recurrent area reflects the degree of clustering of the active neurons in the space defined by the synaptic topology. The theoretically expected ANR values for perfectly clustered or completely unclustered activity depend on the current number $N_{+}^{\text {rec }}$ of active neurons in the recurrent area (dashed orange lines). Note that the ANR is only defined for a non-zero number of active neurons. The intermediate value of the ANR reached after the formation of the $\mathrm{CA}$ reflects the coexistence of an active $\mathrm{CA}$ and single active neurons with few synaptic connections with other active neurons in the recurrent area. $(\mathbf{b}, \mathbf{c}, \mathbf{d})$ The dashed vertical lines indicate the times of the snapshots shown in (a). 

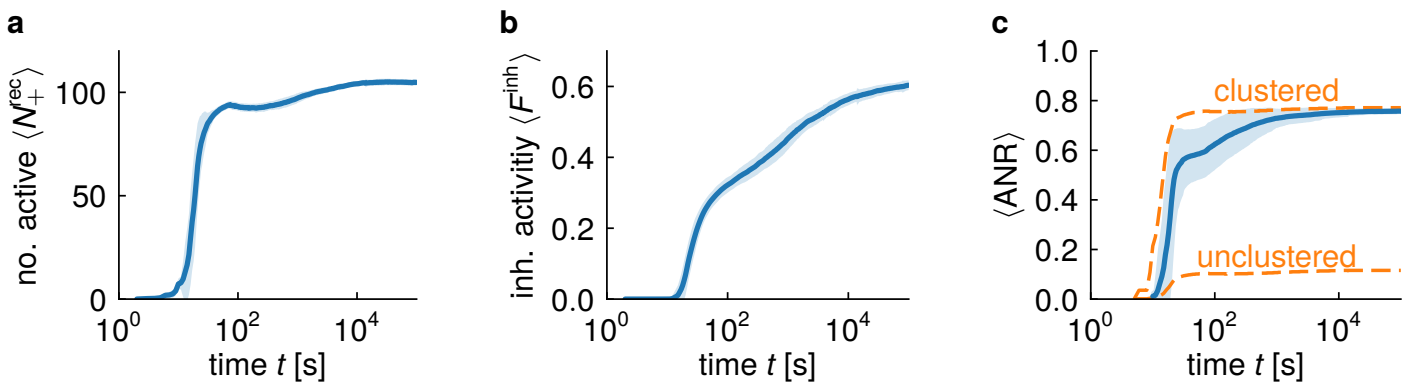

Figure 4.4: Mean long-term development of a CA. A stimulus activating half of the neurons in the input area is presented for $10^{5} \mathrm{~s}$ in 100 independent network instantiations. Note the logarithmic scale of the time axes. The shaded area indicates the standard deviation of the distribution of the respective value within the 100 network instantiations. (a) The mean number $\left\langle N_{+}^{\text {rec }}\right\rangle$ of active neurons in the recurrent area increases slowly after the formation of the CA and reaches a stable value after approximately $10^{4} \mathrm{~s}$. (b) The mean activity $\left\langle F^{\text {inh }}\right\rangle$ of the inhibitory population keeps increasing also for long stimulation times $t$. Due to the direct coupling between the activity in the recurrent area and the membrane potential of the inhibitory population, this directly indicates an increasing sum of excitatory activity in the recurrent area. (c) The mean active neighbor ratio $\langle A N R\rangle$ first reaches an intermediate value in between the theoretically expected values for unclustered activity and clustered activity in the recurrent area. For very long stimulus presentation times, it approaches the limit value for perfectly clustered activity. Here, all active neurons are part of the highly interconnected CA.

by all neurons in the recurrent area (Figure $4.3 \mathrm{~b}$ ). As a result, the number of active neurons within the recurrent area which are not connected to a high number of other active neurons decreases. These neurons do no longer receive enough recurrent excitatory input to counterbalance the inhibitory signals. This leads to a slow increase of the ANR (Figure $4.3 \mathrm{~d}$ ). At the same time, the growing of the CA gets slower and saturates with stronger inhibition which impedes the recruitment of further neurons into the CA ( $t=100 \mathrm{~s}$, see Section 4.2.2).

After the input stimulus is switched off, the activity of the CA drops to baseline $(t=150 \mathrm{~s})$. For the given set of parameters, the recurrent signals in between the neurons which are part of the CA are not sufficient to maintain persistent activity of the CA. However, while the activity of the CA drops, the strong weights of the synapses in between the neurons which are part of the CA remain. As will be shown in section 4.2.3, these modified synaptic weights allow for the recognition and completion of the learned input pattern.

In the example shown here (Figure 4.3), the input stimulus lasts for $125 \mathrm{~s}$. Although the network dynamics has considerably slowed down at the end of this interval, it has not yet reached an equilibrium. In a second analysis, we present the stimulus for a much longer time span of $10^{5} \mathrm{~s}$ to 100 independent network instantiations. We find that after the initial formation of a CA, the mean number $\left\langle N_{+}\right\rangle$of active neurons in the recurrent area continues to slowly increase (Figure $4.4 \mathrm{a}$ ). In parallel, also the mean inhibitory activity $\left\langle F^{\text {inh }}\right\rangle$ (Figure $4.4 \mathrm{~b}$ ) and the mean active neighbor ratio $\langle A N R\rangle$ in the recurrent area (Figure $4.4 \mathrm{c}$ ) increase. For long stimulation times, the value of $\langle$ ANR $\rangle$ gets close to the ANR value expected for perfect clustering of the active neurons. Thus, eventually, all active neurons in the recurrent area are part of the highly interconnected CA.

\subsubsection{Topological Properties}

Whereas the formation of CAs in the presented model is both visually apparent and indicated by the ANR value, the detailed topological properties of the CAs 
remain to be investigated. These properties are the basis for the derivation of a reduced population model in Section 4.3. The population model approximates the mean dynamics inside subpopulations of neurons in the recurrent area. Every such subpopulations has the potential to be recruited as a CA for a given input stimulus. Thus, the properties of the individual subpopulations have to be adjusted to the properties of the CA observed in the numerical solutions of the complete network model. In particular, we require the number of neurons that are recruited into a CA and the mean recurrent and feedforward synaptic connectivity of these neurons.

\section{Number of Neurons in a Cell Assembly}

As discussed in the previous section, the ANR approaches the expected value for perfect clustering for very long presentation times (Figure $4.4 \mathrm{c}$ ). Thus, all active neurons in this limit are part of the CA. Therefore, we define the number $N_{C A}$ of neurons in a CA for a given input stimulus as the limit value of $N_{+}^{\text {rec }}$ for long stimulus presentation times. This number varies only slightly across different network instantiations (Figure $4.4 \mathrm{a}$ ).

Topological and dynamical considerations allow an analytical derivation of $N_{\mathrm{CA}}$. We consider a situation in which a certain stimulus pattern consisting of $N_{+}^{\text {in }}$ active input neurons is presented to the network. We assume that this stimulus has already lead to the formation of a CA in the recurrent area and that this CA has reached its final size $N_{\mathrm{CA}}$. In other words, the final equilibrium of the network state is reached. All $N_{C A}$ neurons in the recurrent area which are part of the CA are highly active, i.e., their equilibrium firing rate $F_{i}^{*}$ is close to 1 . We assume that there are no more unclustered active neurons. All neurons which are not part of the CA are approximated by an equilibrium firing rate $F_{i}^{*} \approx 0$. The equilibrium membrane potential $u^{\mathrm{inh}, *}$ of the inhibitory population is given by the number of neurons in the CA (compare Equation 4.3):

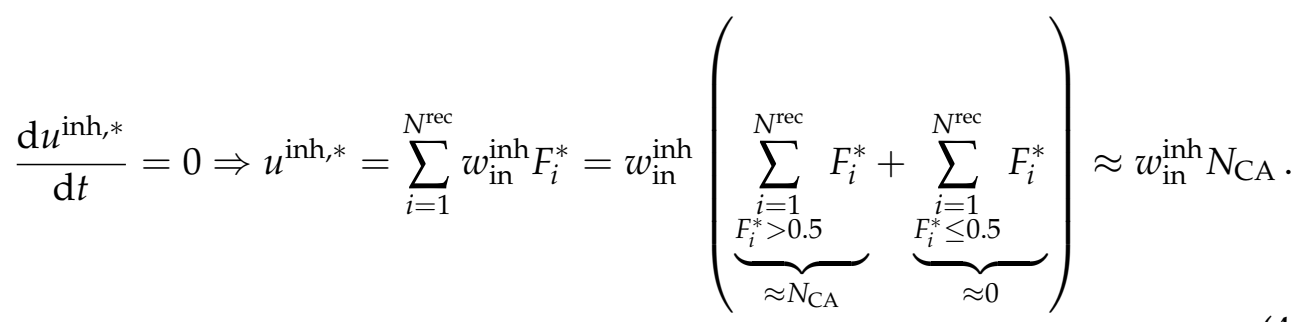

We assume that the network has reached the equilibrium state. This means no more additional neurons in the recurrent area are recruited into the CA. In particular, inactive neurons which are located directly next to the CA do not receive enough excitatory input to cross the inflection point of their firing rate function (Figure $4.5 \mathrm{a}$ ). We can calculate the equilibrium weights of the synapses that project signals from neurons within the CA to these neurons from Equation (4.5). For $F^{\mathrm{T}}=0$, the equilibrium condition $\dot{w}_{i j}^{\text {rec,* }}=0$ leads to $\left(w_{i j}^{\text {rec,* }}\right)^{2}=F_{j}^{*}$. Thus, the weights of all synapses arriving from active presynaptic neurons $\left(F_{j}^{*} \approx 1\right)$ can be approximated by $w_{i j}^{\text {rec,* }} \approx 1$. Similar consideration hold for the feedforward synapses which project signal from active neurons in the input area to inactive neurons in the network (Equation 4.6). From $I_{k}=1$ and $F^{\mathrm{T}}=0$, it follows that $w_{i k}^{\mathrm{ff}, *}=1$. We can therefore approximate the equilibrium value of the membrane potential of a neuron $i$ located 
a

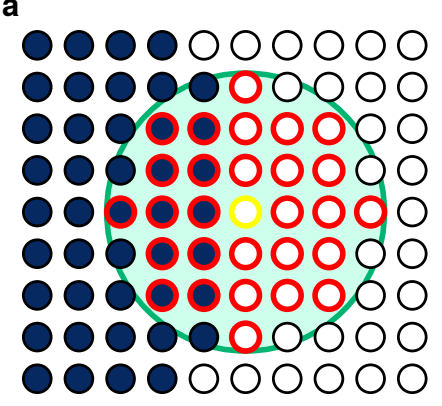

b

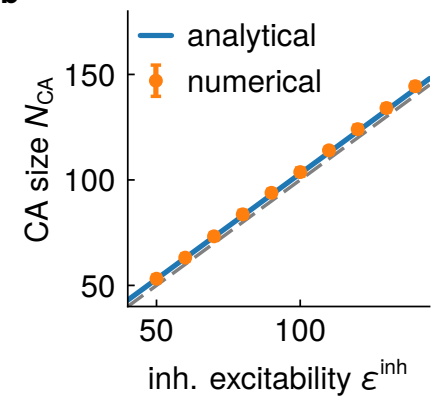

c

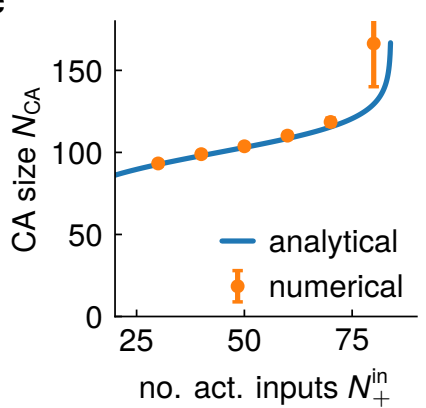

Figure 4.5: Analytical derivation of the CA size and numerical results. (a) The analytical derivation of the number $N_{\mathrm{CA}}$ of neurons in the recurrent area that are recruited for a $\mathrm{CA}$ is based on the excitatory and inhibitory signals received by a neuron (edged in yellow) located right next to the active (dark blue) CA. Based on the recurrent interaction radius $r^{\text {rec }}$ (green) in the recurrent area, such a neuron receives recurrent synaptic signals from $n^{\text {rec }}$ other neurons (edged in red). For estimating the CA size, the number $n_{+}^{\text {rec }}$ of recurrent synaptic signals from active neurons is crucial (dark blue and edged in red). In the case of $r^{\text {rec }}=3$, we find $n_{+}^{\text {rec }}=11$. (b) The analytically derived value of the number $N_{\mathrm{CA}}$ of neurons in the CA matches the mean value obtained in numerical simulations of the network for different values of the inflection point $\epsilon^{\text {inh }}$ of the inhibitory population. The dashed gray line marks the identity $N_{C A}=\epsilon_{\text {inh. }}$. (c) The analytical derived expression for $N_{C A}$ does also correctly capture the influence of different numbers $N_{+}^{\text {in }}$ of active neurons in the input area. Interestingly, $N_{C A}$ does only increase by $34 \%$ when increasing $N_{+}^{\text {in }^{+}}$from 25 to 75. (b, c) For every parameter value, we simulated 100 independent network instantiations. $N_{\text {CA }}$ is given by the number $N_{+}$of active neurons after a stimulus presentation for $10^{5} \mathrm{~s}$. To further accelerate the development of the CA, we reduce the time scales $\tau^{\text {rec }}$ and $\tau^{\text {tf }}$ of the synaptic plasticity processes to $0.1 \mathrm{~s}$. The error bars show the standard deviation among these trials (in many cases too small to be visible).

directly next to the CA (Figure $4.5 \mathrm{a}$ ) as follows (compare Equation 4.1):

$$
\begin{aligned}
& u_{i}^{*}=\sum_{j \in C_{i}^{\text {rec }}} w_{i j}^{\mathrm{rec}, *} F_{j}^{*}+\sum_{k \in C_{i}^{\mathrm{ff}}} w_{i k}^{\mathrm{ff}, *} I_{k}+w_{\mathrm{out}}^{\mathrm{inh}} F^{\mathrm{inh}, *} \\
& =\underbrace{\sum_{\substack{j \in C_{i}^{\text {rec }} \\
F_{j}^{*}>0.5}} w_{i j}^{\mathrm{rec}, *} F_{j}^{*}}_{\approx n_{i,+}^{\mathrm{rec}}}+\underbrace{\sum_{\substack{j \in C_{i}^{\text {rec }} \\
F_{j}^{*} \leq 0.5}} w_{i j}^{\mathrm{rec}, *} F_{j}^{*}+\underbrace{\sum_{\substack{k \in C_{i}^{\mathrm{ff}} \\
I_{k}>0.5}} w_{i k}^{\mathrm{ff}, *} I_{k}}_{\approx n_{i,+}^{\mathrm{ff}}}+\underbrace{\sum_{\substack{k \in C_{i}^{\mathrm{ff}} \\
I_{k} \leq 0.5}} w_{i k}^{\mathrm{ff}, *} I_{k}}_{\approx 0}+w_{\mathrm{out}}^{\mathrm{inh}} F^{\mathrm{inh}, *}}_{\approx 0} \\
& \approx n_{i,+}^{\mathrm{rec}}+n_{i,+}^{\mathrm{ff}}+w_{\mathrm{out}}^{\mathrm{inh}} F^{\mathrm{inh}, *} .
\end{aligned}
$$

Here, $n_{i,+}^{\text {rec }}$ is the number of recurrent synapses transmitting signals from active neurons in the recurrent area to neuron $i$ (blue neurons with red edges in Figure $4.5 \mathrm{a})$. For a recurrent interaction radius $r^{\text {rec }}=3$, geometrical considerations lead to $n_{i,+}^{\text {rec }}=11$ (Figure $4.5 \mathrm{a}$ ). Accordingly, $n_{i,+}^{\mathrm{ff}}=N_{+}^{\mathrm{in}} n^{\mathrm{ff}} / N^{\text {in }}$ is the expected number of synapses transmitting signals from active neurons in the input area to neuron $i$.

From Equation (4.10), we can extract the inhibitory equilibrium firing rate $F^{\text {inh,* }}$ which leads to an equilibrium membrane potential $u_{i}^{*}$ of a neuron located directly next to the CA. Using the inverse of the firing rate function of the inhibitory population (Equation 4.4), we can transform $F^{\mathrm{inh}, *}$ into the respective equilibrium membrane potential $u^{\mathrm{inh}, *}$. Finally, using the expression for $u^{\mathrm{inh}, *}$ in Equation (4.10), we arrive at a formula which gives the number $N_{C A}$ of neurons in the CA required to keep the inactive neuron $i$ at a membrane potential $u_{i}^{*}$. As $u_{i}^{*}=\epsilon$ defines the steepest point of the firing rate function and marks the transition from inactivity to activity, we evaluate the formula for this condition. This yields an expression for the number $N_{C A}$ 
of neurons which are recruited into the CA:

$$
N_{\mathrm{CA}}=\frac{1}{w_{\text {in }}^{\text {inh }}}\left(\epsilon^{\mathrm{inh}}-\frac{1}{\beta^{\text {inh }}}\left(\log \left(\frac{w_{\text {out }}^{\text {inh }}}{\epsilon-n_{i,+}^{\text {rec }}-n_{i,+}^{\text {ff }}}-1\right)\right)\right) .
$$

For deriving the expression in Equation (4.12), we assumed the weights of all synapses to be close to the respective equilibrium state. Therefore, a comparison of the values obtained from Equation (4.12) with the results from solving the network dynamics numerically requires long simulation times. These long-run simulations confirm the linear relationship between the position of the inflection point $\epsilon_{\text {inh }}$ and the CA size $N^{\mathrm{CA}}$ predicted by Equation (4.12) (Figure $4.5 \mathrm{~b}$ ). Note that the offset of the curve from the diagonal is comparably small indicating small values of the logarithm in Equation (4.12) $\left(\beta^{\text {inh }}=1\right)$. The influence of variations of the parameters which occur in the argument of this logarithm on the number of neurons in the CA is small. In particular, this holds for the expected number $n_{i,+}^{\mathrm{ff}}$ of signals received from active neurons in the input area. The value of $n_{i,+}^{\mathrm{ff}}$ is directly proportional to the total number $N_{+}^{\text {in }}$ of active neurons in the input area: $n_{i,+}^{\mathrm{ff}}=n^{\mathrm{ff}} N^{\mathrm{in}} / n^{\mathrm{ff}}$. Accordingly, we find $N_{\mathrm{CA}} \approx 90$ for $N^{\text {in }}=25$ and $N_{\mathrm{CA}} \approx 121$ for $N^{\text {in }}=75$ (Figure $4.5 \mathrm{c}$ ). Thus, an increase of $N_{+}^{\text {in }}$ of $200 \%$ leads to an increase of only approximately $34 \%$ of $N_{+}^{\text {rec }}$. This shows that the system is able to form memory representations with almost uniform properties for different stimulus sizes.

\section{Number of Active Feedforward and Recurrent Connections}

The collective dynamical properties of the neurons within a CA are determined by both the recurrent and the feedforward connectivity of the neurons within the CA. The numbers of the recurrent and feedforward synapses transmitting signals from active neurons to CA neurons are crucial parameters for the population model in Section 4.3. Therefore, here, we study these numbers for the active neurons in the recurrent area.

We denote by $\bar{n}_{++}^{\mathrm{ff}}$ the mean of all values $n_{i,+}^{\mathrm{ff}}$ of neurons $i$ whose firing rate $F_{i}$ is larger than 0.5 , i.e., which are active. Similarly, we define $\bar{n}_{++}^{\text {rec }}$ as the mean of all values $n_{i,+}^{\text {rec }}$ of active neurons $i$. In the very early CA development phase, only neurons $i$ in the recurrent area with a high number of feedforward synapses from active neurons in the input area receive enough excitation to get activated (Figure $4.6 \mathrm{a}$ ). This results in a high value of $\bar{n}_{++}^{\mathrm{ff}}$. As discussed in Section 4.2.1, these initially activated neurons in the recurrent are unclustered. Accordingly, they receive few inputs from other active neurons in the recurrent area and the value of $\bar{n}_{++}^{\text {rec }}$ is low. During the formation of the CA, the relation of $\bar{n}_{++}^{\mathrm{ff}}$ and $\bar{n}_{++}^{\text {rec }}$ starts to reverse. The increasing clustering of the active neurons in the recurrent area (Figure $4.4 \mathrm{c}$ ) leads to a higher number of synapses transmitting signals in between active neurons. Thus, $\bar{n}_{++}^{\text {rec }}$ starts to increase. At the same time, also neurons receiving fewer synapses from active neurons in the input area are recruited into the CA and $\bar{n}_{++}^{\mathrm{ff}}$ starts to decrease. This development reflects the transition from an unclustered representation of the input stimulus which only relies on feedforward signals to a representation by a CA.

As shown in Section 4.2.1, for long stimulus presentation times, the ANR approaches the theoretical expected value for perfect clustering. Thus, in this limit, all active neurons are part of the formed CA and the numbers $\bar{n}_{++}^{\mathrm{ff}}$ and $\bar{n}_{++}^{\text {rec }}$ characterize the connectivity of this CA. We find that the mean number $\bar{n}_{++}^{\mathrm{ff}}$ of synapses from active input neurons received by CA neurons varies only slightly among the different initializations of the network model (Figure $4.6 \mathrm{~b}$ ). The average value after $10^{5} \mathrm{~s}$ of 
a

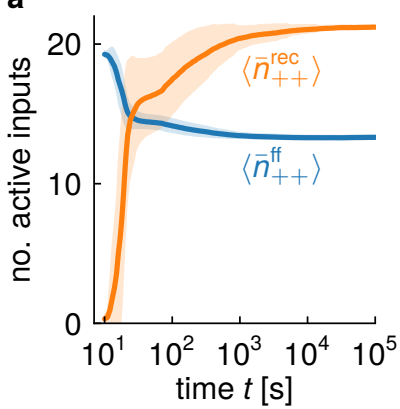

b

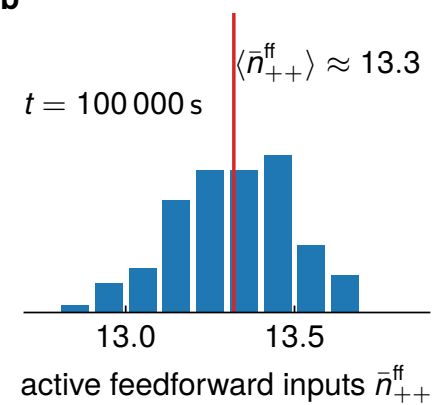

C

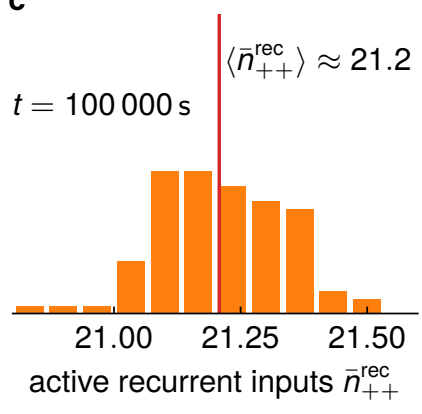

Figure 4.6: Mean number of active recurrent and feedforward inputs for CA neurons. The value $\bar{n}_{++}^{\mathrm{ff}}$ is given by the mean of the numbers of incoming active feedforward inputs for active neurons in the recurrent area. Similarly, $\bar{n}_{++}^{\text {rec }}$ is the mean number of active recurrent inputs received by active neurons in the recurrent area. For long stimulation durations, these values characterize the connectivity of the formed CA and are of relevance for the population model derived in Section 4.3. (a) The relation between $\left\langle\bar{n}_{++}^{\text {rec }}\right\rangle$ and $\left\langle\bar{n}_{++}^{\mathrm{Hf}}\right\rangle$ is reversed during the formation of a CA. This reflects the transition from a feedforward dominated representation of the input stimulus to a representation by a CA. As for long stimulus presentation durations all active neurons in the recurrent area are part of the CA, in this limit, $\left\langle\bar{n}_{++}^{\text {rec }}\right\rangle$ and $\left\langle\bar{n}_{++}^{\mathrm{ff}}\right\rangle$ describe the connectivity of the CA. The shaded area gives the standard deviation among 100 independent network instantiations. (b) The value of $\left\langle\bar{n}_{++}^{\mathrm{ff}}\right\rangle$ converges toward the mean number of incoming feedforward synapses from active neurons in the input area which a neuron within the CA receives. (c) The value of $\left\langle\bar{n}_{++}^{\text {rec }}\right\rangle$ corresponds to the mean number of incoming recurrent synapses which a neuron in the CA receives from other neurons in the CA. $(\mathbf{a}, \mathbf{b}, \mathbf{c})$ The data is extracted from the same 100 long-term network simulations as used in Figure 4.4. (b, c) The connectivity data is extracted after a stimulus presentation duration of $10^{5} \mathrm{~s}$. The red line indicates the mean of the distribution.

stimulus presentation is $\left\langle\bar{n}_{++}^{\mathrm{ff}}\right\rangle \approx 13.3$. The standard deviation of the underlying distribution is $\sigma\left(\bar{n}_{++}^{\mathrm{ff}}\right) \approx 0.2$. Every neuron in the recurrent area receives synapses from $n^{\mathrm{ff}}=25$ randomly chosen neurons in the input area. During the presentation of the stimulus, half of these neurons in the input area are active. Thus, we would expect the ensemble average of $n_{++}^{\mathrm{ff}}$ to be 12.5 if the CA would be formed at a random location within the recurrent area. Instead, the presentation of an input stimulus leads to a competition among the neurons in the recurrent area about representing this stimulus. In this competition, the recruitment of neurons which receive a higher number of signals from the active neurons in the input area is more likely. We have to include the resulting larger mean value $\left\langle\bar{n}_{++}^{\mathrm{ff}}\right\rangle$ when setting up the population model in Section 4.3.

The distribution of the mean number $\bar{n}_{++}^{\text {rec }}$ of incoming recurrent synapses from active neurons after long stimulus presentations has a mean of $\left\langle\bar{n}_{+}^{\text {rec }}\right\rangle \approx 21.2$ (Figure $4.6 \mathrm{c})$. The standard deviation of this distribution is $\sigma\left(\bar{n}_{+}^{\text {rec }}\right) \approx 0.1$.

\subsubsection{Pattern Completion and Separation}

The topological properties of a CA directly influence its functional properties. The most prominent functional property commonly attributed to CAs is their capability to perform pattern completion and separation. Pattern completion occurs if two input stimuli are mapped to memory representations whose difference is smaller than the difference of the input stimuli. Pattern separation, in contrast, refers to a mapping of two input stimuli to two memory representations with a higher difference than the one of the input stimuli.

We investigate the pattern completion and separation capabilities of the memory representations formed in the here described model. For this, we present a stimulus 
which activates half of the neurons in the input layer to the network. The active neurons in the input area define the set $S_{\mathrm{A}}$. This stimulus is presented to the network for a time span $t_{\text {learn. }}$. The active neurons in the recurrent area at the end of this learning phase define the set $R_{\mathrm{A}}$. Thus, $R_{\mathrm{A}}$ is the memory representation of the stimulus $S_{\mathrm{A}}$. After a short period without activity in the input area, a recall stimulus $S_{\mathrm{B}}$ is presented for a short time span $t_{\text {recall }}\left(t_{\text {recall }} \ll t_{\text {learn }}\right)$. The stimulus $S_{\mathrm{B}}$ consists of the same number of neurons in the input area as the stimulus $S_{\mathrm{A}}\left(\left|S_{\mathrm{A}}\right|=\left|S_{\mathrm{B}}\right|\right)$. The activity of the recurrent area at the end of this recall phase defines the recalled representation $R_{\mathrm{B}}$. As outlined in Section 4.1.2, the difference between the Jaccard index $J\left(S_{\mathrm{A}}, S_{\mathrm{B}}\right)$ of the two stimuli and the Jaccard index $J\left(R_{\mathrm{A}}, R_{\mathrm{B}}\right)$ of the corresponding representations in the recurrent area determines if pattern completion or pattern separation has occurred.

In the following, first, we investigate the pattern completion and pattern separation properties of the network that rely solely on neuronal dynamics. In a second step, we allow the synaptic plasticity processes to be also active during recall.

\section{Static Recall}

In a first experiment, similar to previous studies (e.g. Hopfield, 1982; Blumenfeld et al., 2006), we artificially switch off synaptic plasticity at both the feedforward and the recurrent synapses after the learning phase (Figure 4.7 a). Typical recall processes are assumed to occur on time scales short enough to ignore the influence of plasticity process. This way, we can analyze the pattern completion and separation properties relying solely on the dynamics of the neuronal activities.

For learning durations $t_{\text {learn }}$ which are significantly shorter than the typical stimulation time required to build up a CA $\left(t_{\text {learn }} \lesssim 20\right)$, the network performs pattern separation $\left(J\left(R_{\mathrm{A}}, R_{\mathrm{B}}\right)<J\left(S_{\mathrm{A}}, S_{\mathrm{B}}\right)\right)$ for the whole range of stimulus overlaps $J\left(S_{\mathrm{A}}, S_{\mathrm{B}}\right)$ $\left(t_{\text {learn }}=10 \mathrm{~s}\right.$ in Figure $\left.4.7 \mathrm{a}\right)$. For these values of $t_{\text {learn }}$, the sizes of the representations formed in the recurrent area are small and almost exclusively determined by the random feedforward connectivity. As the weights of the feedforward synapses are close to their initial values, only few memory neurons receive enough stimulation to reach the inflection point of the firing rate function, i.e., to become active. Additionally, the active neurons are not yet clustered and the recurrent weights are low preventing effective recurrent interactions. Small changes in the input stimulus can easily deactivate active neurons and vice versa. These changes in the neuronal activity imply differences in the respective memory representations which yield a low similarity $J\left(R_{\mathrm{A}}, R_{\mathrm{B}}\right)$ of the two representations.

If we increase the learning duration $t_{\text {learn }}$ to $t_{\text {learn }}=20 \mathrm{~s}$, the $J\left(S_{\mathrm{A}}, S_{\mathrm{B}}\right)-J\left(R_{\mathrm{A}}, R_{\mathrm{B}}\right)$ curve is shifted close to the identity line (Figure $4.7 \mathrm{a}$ ). This is due to the increased number of neurons representing the learned stimulus and receiving inputs from potentiated feedforward synapses (see Figure $4.3 \mathrm{a}$ ). At the same time, in some network instantiations a CA has already emerged at this point of time. This is also the reason of the high standard deviation of the $J\left(S_{\mathrm{A}}, S_{\mathrm{B}}\right)-J\left(R_{\mathrm{A}}, R_{\mathrm{B}}\right)$-curve for this learning duration.

We can observe the effect of the presence of a CA on the $J\left(S_{\mathrm{A}}, S_{\mathrm{B}}\right)-J\left(R_{\mathrm{A}}, R_{\mathrm{B}}\right)$-curve by analyzing this curve for a learning duration $t_{\text {learn }}$ at which a CA has been formed in almost all network instantiations ( $t_{\text {learn }}=30 \mathrm{~s}$ in Figure $\left.4.7 \mathrm{a}\right)$. Now, the network, or rather the CA, performs pattern completion $\left(J\left(R_{\mathrm{A}}, R_{\mathrm{B}}\right)>J\left(S_{\mathrm{A}}, S_{\mathrm{B}}\right)\right)$ for a wide range of stimulus similarities $J\left(S_{\mathrm{A}}, S_{\mathrm{B}}\right) \gtrsim 0.15$. As soon as neurons in the recurrent area which are part of the $C A$ receive enough feedforward signals to become active, 
a

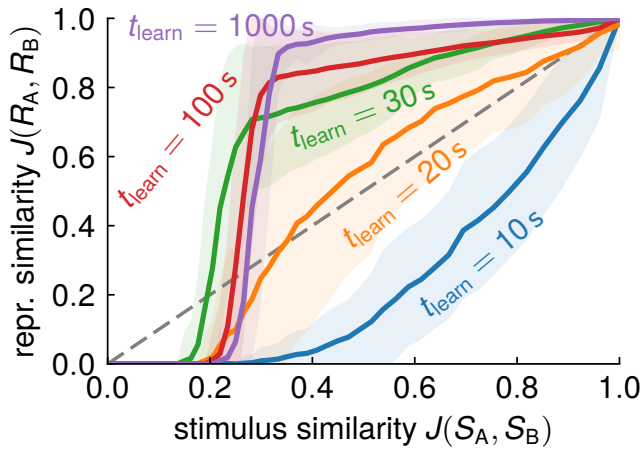

b

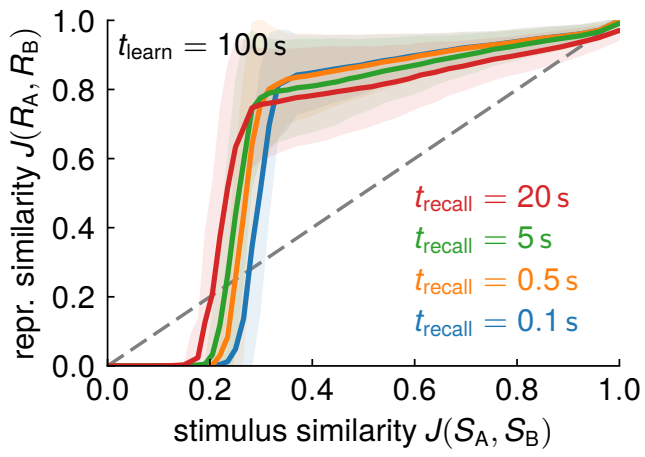

Figure 4.7: Pattern completion and separation properties of the network model. A stimulus $S_{\mathrm{A}}$ defined by a set of active neurons in the input area is present for a duration $t_{\text {learn }}$ to the network. The neurons in the recurrent area which are active at the end of this time span define the stimulus representation $R_{\mathrm{A}}$. Afterwards, a recall stimulus $S_{\mathrm{B}}$ with similarity $J\left(S_{\mathrm{A}}, S_{\mathrm{B}}\right)$ to $S_{\mathrm{A}}$ is presented to the network for a duration $t_{\text {recall. }}$. The activity in the recurrent area at the end of this time span defines the recalled representation $R_{\mathrm{B}}$. Pattern completion occurs if $J\left(R_{\mathrm{A}}, R_{\mathrm{B}}\right)>J\left(S_{\mathrm{A}}, S_{\mathrm{B}}\right)$. Pattern separation is defined as $J\left(R_{\mathrm{A}}, R_{\mathrm{B}}\right)<J\left(S_{\mathrm{A}}, S_{\mathrm{B}}\right)$ (a) We switch off the plasticity before presenting the recall stimulus. Thus, the recall is only based on activity dynamics. The mean similarity between $R_{\mathrm{A}}$ and $R_{\mathrm{B}}$ depends not only on the similarity of $S_{A}$ and $S_{B}$ but also on the time span $t_{\text {learn }}$. For short learning times $\left(t_{\text {learn }}=10 \mathrm{~s}\right)$, the network performs pattern separation for all values of $J\left(S_{A}, S_{B}\right)$. For longer learning times $\left(t_{\text {learn }} \gtrsim 30 \mathrm{~s}\right)$, pattern completion occurs for a broad range of $J\left(S_{A}, S_{B}\right)$ values. In all cases, we use $t_{\text {recall }}=5 \mathrm{~s}$. (b) After the stimulus presentation for $t_{\text {learn }}=100 \mathrm{~s}$, we keep the synaptic plasticity processes active also for the recall phase. Synaptic plasticity assists the process of pattern completion. For longer durations $t_{\text {recall }}$ of the recall stimulus presentation, the border between pattern separation and pattern completion is shifted toward lower stimulus similarities. $(\mathrm{a}, \mathrm{b})$ The dashed grey line indicates the identity $J\left(S_{\mathrm{A}}, S_{\mathrm{B}}\right)=J\left(R_{\mathrm{A}}, R_{\mathrm{B}}\right)$.

they excite other neurons of the CA via the strong recurrent synaptic connections. This eventually leads to the activation of the complete CA.

Shortly after the formation of the CA, there is still a number of active neurons in the recurrent area that are not part of the CA, i.e., not part of the cluster of active neurons. These unclustered active neurons vanish during an ongoing presentation of the learning stimulus ( $t_{\text {learn }}=1000 \mathrm{~s}$ in Figure $4.7 \mathrm{a}$ ). This sharpens the pattern completion effect: The representation similarity $J\left(R_{\mathrm{A}}, R_{\mathrm{B}}\right)$ reaches values close to one for a wide range of stimulus similarities $J\left(S_{\mathrm{A}}, S_{\mathrm{B}}\right)$. Interestingly, also the regime for low stimulus similarities $J\left(S_{\mathrm{A}}, S_{\mathrm{B}}\right)$ in which pattern separation is performed increases for long stimulation durations. Now, pattern completion occurs only for $J\left(S_{\mathrm{A}}, S_{\mathrm{B}}\right) \gtrsim$ 0.25 . This is due to the downscaling of feedforward synapses from inactive neurons in the input area to neurons in the CA due to the synaptic scaling term of the plasticity rule in Equation (4.6). Thus, neurons in the input area which are part of $S_{\mathrm{B}}$ but not of $S_{\mathrm{A}}$ cannot contribute to the activation of the memory representation $R_{\mathrm{A}}$.

In conclusion, the network model initially performs pattern separation for all input stimulus similarities. The formation of a CA which represents the learned stimulus in the recurrent area, in contrast, leads to pattern completion for a broad range of stimulus similarities. Longer stimulation times during the CA learning phase shift the border between pattern separation and pattern completion toward higher stimulus similarities. At the same time, long stimulus durations pronounce the pattern completion effect for mediate to high stimulus similarities. 


\section{Plastic Recall}

As discussed above, most studies of neuronal network models of memory assume synaptic plasticity to be only active during the learning phase. In living neuronal systems, however, plasticity is an ongoing dynamic process which influences the activity dynamics both during learning and during recall. Therefore, we now keep the synaptic plasticity processes in the here described network model active during the recall phase. We find that the representation similarity $J\left(R_{\mathrm{A}}, R_{\mathrm{B}}\right)$ in the presence of a CA depends on the duration $t_{\text {recall }}$ of the recall interval (Figure $4.7 \mathrm{~b}$ ). For small values of $t_{\text {recall, }}$, the influence of synaptic plasticity is negligible and the recall of the CA solely relies on the faster neuronal activity dynamics.

In contrast, for longer recall intervals, synaptic plasticity supports the pattern completion process induced by the recall stimulus. While a stimulus similarity of $J\left(S_{\mathrm{A}}, S_{\mathrm{B}}\right)=0.25$ leads to almost complete pattern separation $\left(J\left(R_{\mathrm{A}}, R_{\mathrm{B}}\right) \approx 0.05\right)$ for $t_{\text {recall }}=0.1 \mathrm{~s}$, it results in pattern completion $\left(J\left(R_{\mathrm{A}}, R_{\mathrm{B}}\right) \approx 0.63\right)$ for $t_{\text {recall }}=20 \mathrm{~s}$. The ongoing synaptic plasticity leads to the potentiation of feedforward synapses transmitting signals from neurons in the input area which belong to $S_{\mathrm{B}}$ but not to $S_{\mathrm{A}}$. The increasing weights of these synapses result in to stronger excitatory signals received by the neurons in the recurrent area. This, in turn, may lead to the activation of a CA which beforehand did not receive enough excitatory input signals to become active. As a result, the transition between pattern separation and pattern completion is shifted toward lower values of $J\left(S_{\mathrm{A}}, S_{\mathrm{B}}\right)$ for longer recall times $t_{\text {recall }}$. This effect saturates for long recall intervals $t_{\text {recall }}$.

Ongoing synaptic plasticity during the recall phase supports pattern completion. Still, it is not possible to activate the originally formed CA with recall stimuli with very low similarity to the original stimulus $\left(J\left(S_{\mathrm{A}}, S_{\mathrm{B}}\right) \lesssim 0.2\right)$. Instead, in these cases, the ongoing synaptic plasticity results in the formation of a second $C A$. This new CA explicitly represents the recall stimulus $S_{\mathrm{B}}$. In the following, we investigate the formation of a second CA in more detail.

\subsubsection{Formation of Multiple Cell Assemblies}

The formation of a first CA in the recurrent area may influence the initial conditions for the formation of further CAs representing different stimuli. In the following, we refer to the set of neurons in the recurrent are which are part of the first CA as population 1 . The stimulus which lead to the formation of the CA in population 1 is called stimulus $A$. The presentation of a second stimulus $B$ leads to the formation of a new CA if the similarity between stimulus A and stimulus B is small enough (compare Figure $4.7 \mathrm{~b}$ ). We refer to the neurons which are recruited for this new CA as population 2. Additionally, we introduce a control population. This control population contains all neurons in the recurrent area which belong to neither of the two CA populations.

Interestingly, population 1 and population 2 do not share any common neurons (Figure $4.8 \mathrm{a}$ ). The competition introduced by the synaptic scaling term of the plasticity rule of the recurrent synapses (Equation 4.5) prevents overlaps. The time course of the activity of the inhibitory population during the formation of the CA in population 2 is highly similar to the one of the inhibitory activity during the formation of the CA in population 1 (Figure $4.8 \mathrm{~b}$ ). The same is true for the time course of the number of active neurons (Figure $4.8 \mathrm{c}$ ) and the active neighbor ratio in the network (Figure $4.8 \mathrm{~d}$ ). Thus, the formation of additional CAs is qualitatively equivalent to the formation of the first CA in a blank network. 
a
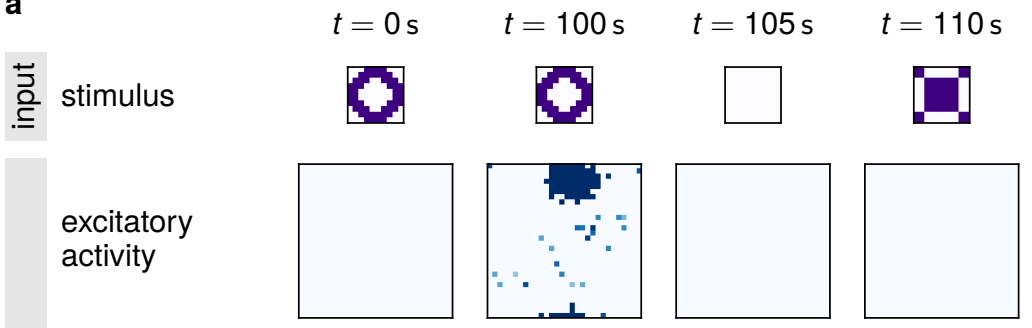

$t=210 \mathrm{~s}$

\begin{tabular}{|l} 
mean incoming \\
recurrent \\
weight
\end{tabular}
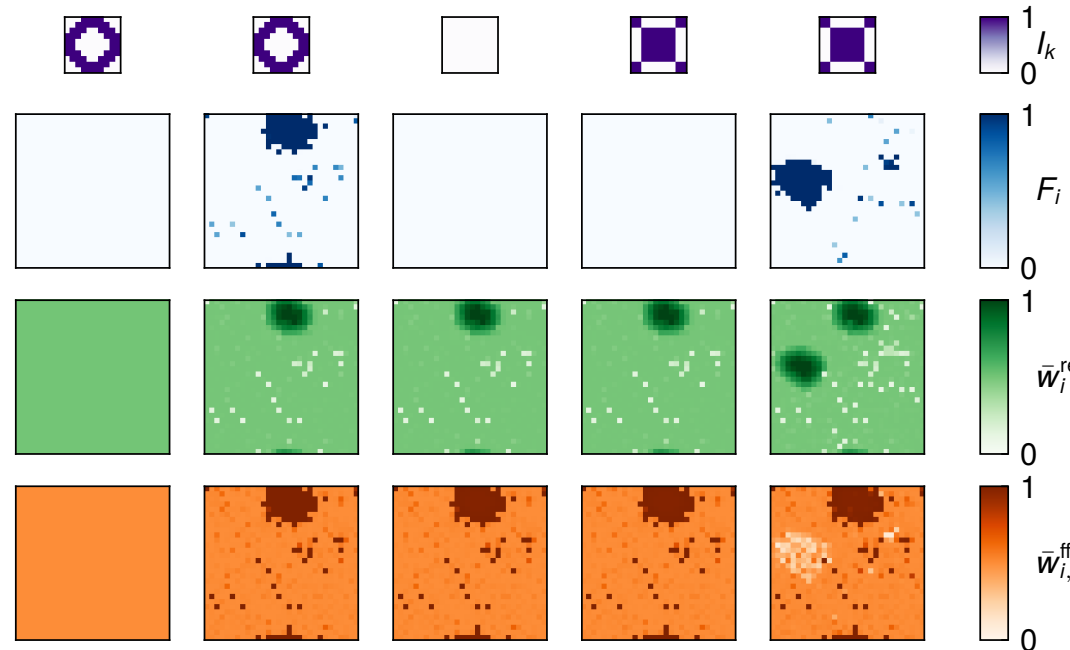

mean incoming

feedforward

weight from

stimulus $B$
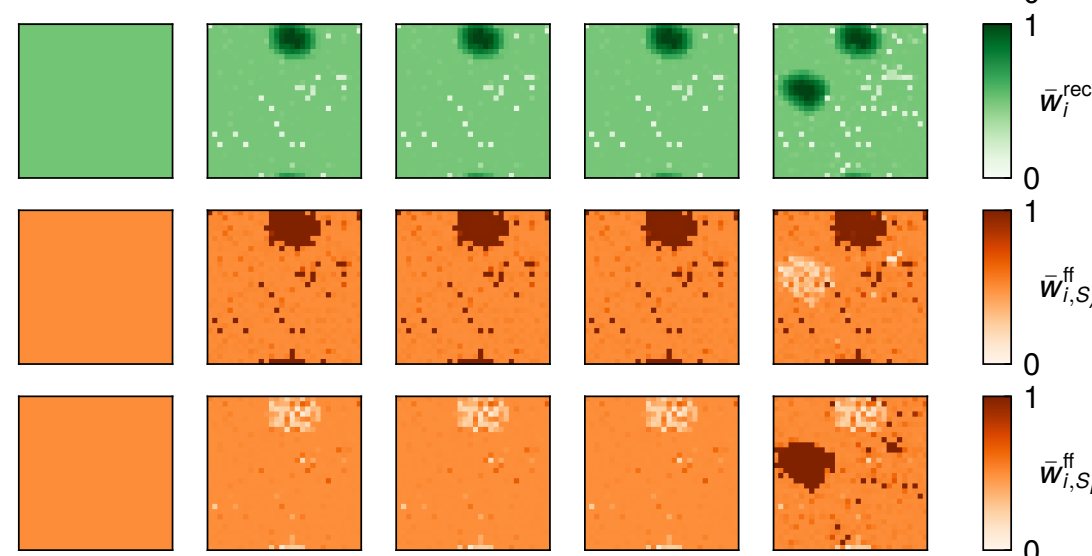

b

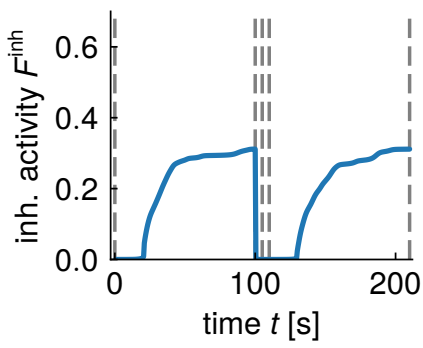

C

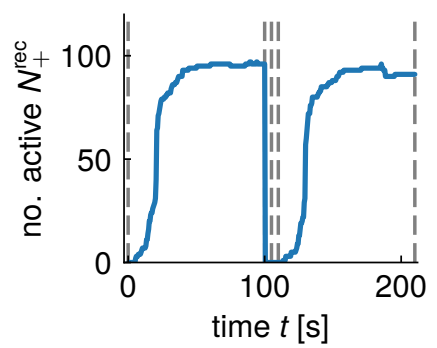

d

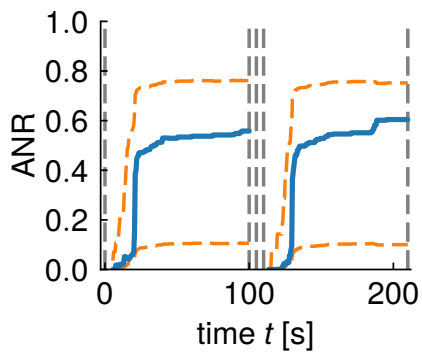

Figure 4.8: Formation of a second self-organized CA in the network model. Two different stimuli are presented successively to the network. Stimulus $A$ is active for $0 \mathrm{~s}<t<100 \mathrm{~s}$. Stimulus $B$ is active for $110 \mathrm{~s}<t<210 \mathrm{~s}$. Both stimuli contain 52 out of the 100 stimulus neurons of which they share 12 . The stimulus similarity is thus $J\left(S_{A}, S_{B}\right)=12 / 92 \approx 0.13$. (a) Every stimulus leads to the formation of a CA in the recurrent area. The two CAs are formed at non-overlapping locations and both CAs are characterized by strong recurrent synapses. Feedforward synapses originating from the stimulus that is represented by the CA are potentiated. Feedforward synapses from neurons in the input area that belong to the respective other stimulus are scaled down. (b) The time course of the activity $F^{\text {inh }}$ of the inhibitory population during the formation of the first CA resembles the time course during the formation of the second CA. (c) Also the time course of the number $N^{\text {rec }}$ of active neurons in the recurrent area and (d) the time course of the active neighbor ratio (ANR) during the two CA formation processes does not show significant differences. The dashed orange lines give the expected ANR for perfectly clustered and completely unclustered activity depending on the current number $N_{+}^{\text {rec }}$ of active neurons. Note that the ANR is only defined for a non-zero number of active neurons. (b, c, d) The dashed vertical lines indicate the times of the network plots shown in (a). 

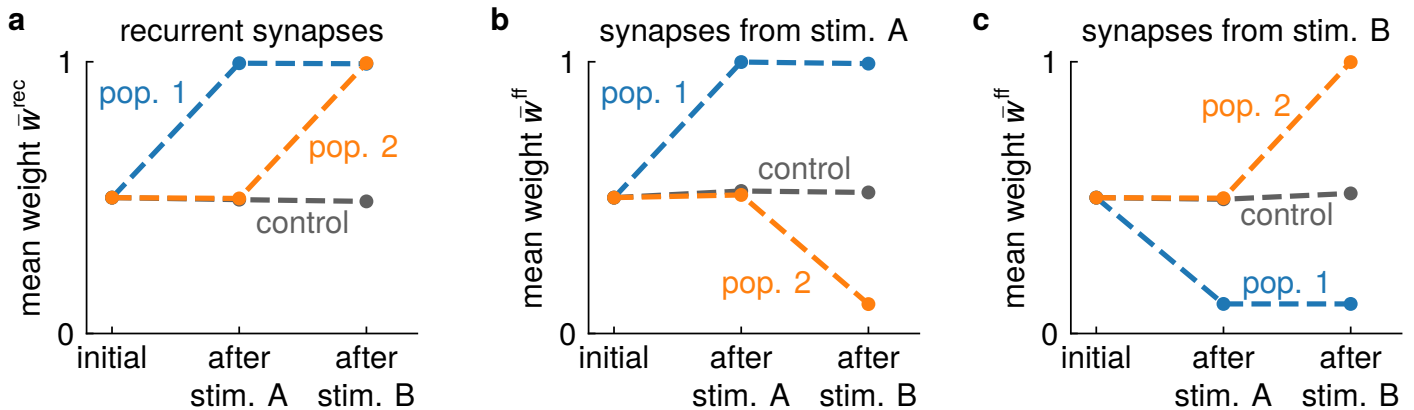

Figure 4.9: Plasticity of feedforward and recurrent synapses during the sequential formation of two CAs. Two non-overlapping stimuli $A$ and $B$ each consisting of 50 stimulus neurons are successively presented for $100 \mathrm{~s}$ each to the network. All neurons which are active at the end of the presentation of stimulus $A$ are considered as population 1. All neurons which are active at the end of the presentation of stimulus B are part of population 2. Neurons which belong neither to population 1 or population 2 are part of the control group. The shown mean synaptic weights are averaged over 100 independent network initializations. The dashed lines are visual aids and do not represent the actual time courses. (a) During the formation of a CA in one of the two populations, the weights of recurrent synapses in the respective population are potentiated. The weights of recurrent synapses in the other populations are not modified. (b) The formation of a CA in population 1 leads to the potentiation of the feedforward synapses transmitting signals from neurons in the input area which are part of stimulus $A$ to the neurons within population 1. Feedforward synapses from the same neurons in the input area to other neurons in the recurrent area are not modified. The formation of a CA in populations 2 results in the downscaling of the feedforward synapses from stimulus A neurons in the input area to the neurons belonging to population 2 in the recurrent area. (c) The weights of the feedforward synapses originating from stimulus $B$ neurons and transmitting signals to neurons within population 1 are downscaled during the formation of a CA in population 1. The weights of feedforward synapses connecting neurons in the input area which belong to stimulus $B$ with neurons in the recurrent area which are part of population 2 are potentiated during the formation of a CA in population 2. Figure adapted from Auth et al., (2017).

During the presentation of stimulus $\mathrm{A}$, the recurrent synapses in population 1 are potentiated (Figure $4.9 \mathrm{a}$ ). Accordingly, during the presentation of stimulus B, the recurrent synapses in population 2 are potentiated. In both cases, the recurrent synapses in the respective other population and in the control population are not significantly modified. Similarly, also the synaptic plasticity of feedforward synapses results in significant weight changes only at the synapses that converge at the population which is recruited for representing the current stimulus (Figures $4.9 \mathrm{~b}$ and $4.9 \mathrm{c})$. Synapses transmitting signals from the active neurons in the input areas to the active population in the recurrent area are potentiated. In parallel, synapses transmitting signals from inactive neurons in the input area to the active population are scaled down by the homeostatic synaptic scaling term of the plasticity rule. As will be outlined in Section 4.3.4, this downscaling is crucial for the discrimination of the currently learned stimulus from other stimuli.

In summary, the network model allows for the formation of multiple distinct CAs in the recurrent area. During the formation of a CA, the recurrent and feedforward synapses of other populations in the recurrent area are not significantly modified. Thus, the formation of a new CA does neither disrupt existing CAs nor prevent the formation of additional CAs. Of course, this holds only as long as the number of neurons which are not recruited for any $\mathrm{CA}$ is high enough to allow for the formation of a new CA. 


\subsection{Population Model}

The network model investigated in the previous section is a high-dimensional dynamical system. The list of its dynamic variables includes the membrane potentials of the 900 neurons in the recurrent area, the membrane potential of the inhibitory population, the weights of $900 \times 28=25200$ recurrent synapses, and the weights of the $900 \times 25=22500$ feedforward synapses. In total, there are 48601 dynamic variables in the model. This prevents a direct analytical treatment of the underlying dynamics in terms of its attractive and repulsive equilibria. In this section, we develop a population model which reduces the network dynamics to a set of seven interacting dynamical equations. This reduction allows the derivation of the equilibria and bifurcations underlying the self-organized formation and allocation of CAs in the network. These equilbria and bifurcations determine the long-run behavior of the system. Therefore, they allow us to obtain a more detailed understanding of the self-organized interplay of the plasticity at the recurrent and feedforward synapses in the limit of long stimulation times. Furthermore, we can draw conclusions about the components of the model which are essential for making the self-organized formation and allocation of CAs possible.

\subsubsection{Model Definition}

The population model is based on two distinct neuronal populations which both have the potential to represent a given input stimulus (Figure 4.10). We assume the two populations to be locally separated in the recurrent area such that there is effectively no interaction via recurrent synapses in between them. Their only way of interaction is by means of the inhibitory population. We describe each of the two populations by a mean membrane potential $\bar{u}_{i}, i \in\{1,2\}$. Differences between neurons at different positions within the populations are neglected.

In addition to the inhibitory signals, the neurons within each population receive recurrent excitation from other neurons within the same population and external inputs from two different input stimuli $\mathrm{A}$ and $\mathrm{B}$. The mean membrane potential $\bar{u}_{i}$, $i \in\{1,2\}$, in each of the two populations is described by

$$
\tau \frac{\mathrm{d} \bar{u}_{i}}{\mathrm{~d} t}=-\bar{u}_{i}+\bar{n}_{++}^{\mathrm{rec}} \bar{w}_{i}^{\mathrm{rec}} \bar{F}_{i}+w_{\mathrm{out}}^{\mathrm{inh}} F^{\mathrm{inh}}+\sum_{k=A, B} \bar{n}_{++}^{\mathrm{ff}} \tau \bar{w}_{i k}^{\mathrm{ff}} \bar{I}_{k} .
$$

The time scale $\tau$ and the weight $w_{\text {out }}^{\text {inh }}$ correspond to the equivalent values in the network simulation. The mean input strength $\bar{I}_{k}, k \in\{A, B\}$, describes the mean firing rate of the active neurons within the input area which belong to the respective stimulus. If not stated otherwise, $\bar{I}_{k}$ takes values of either one or zero. The number $\bar{n}_{++}^{\text {rec }}$ describes the mean number of recurrent synapses received by a neuron within one of the two populations from other neurons belonging to the same population. It corresponds to the mean number $\bar{n}_{++}^{\text {rec }}$ of synaptic signals in between active neurons obtained for long stimulation durations in the previous section (Figure 4.6c). Accordingly, $\bar{n}_{++}^{\mathrm{ff}}$ gives the mean number of incoming feedforward synapses from active neurons in the input area received by neurons within one of the two populations. For this, we use the value $\bar{n}_{++}^{\mathrm{ff}}$ obtained for long stimulation durations in the complete network model (Figure 4.6b). As in the full network simulations, we transform the mean membrane potential $\bar{u}_{i}$ of a population into a mean firing rate $\bar{F}_{i}$ through the 


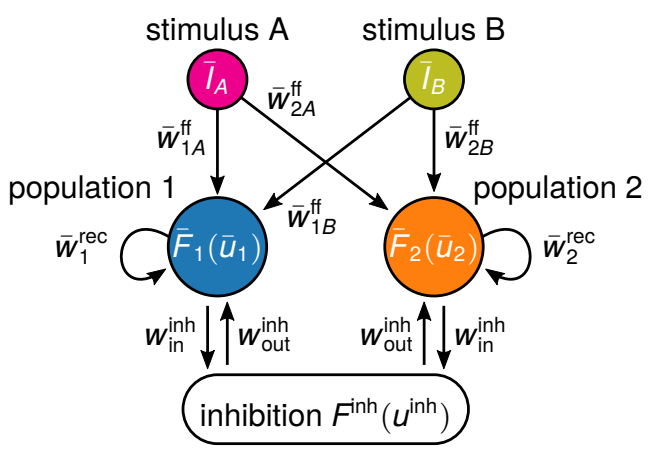

Figure 4.10: Structure of the population model. The two populations 1 and 2 are described by their mean membrane potentials $\bar{u}_{i}, i \in\{1,2\}$, and the mean weight $\bar{w}_{i}^{\text {rec }}$ of their internal recurrent synapses. The populations receive feedforward signals from two input stimuli with mean activity $\bar{I}_{k}, k \in\{A, B\}$, via synapses with mean weights $\bar{w}_{i k}^{\mathrm{ff}}$. The only interaction between the two populations is mediated by the inhibitory population with membrane potential $u^{\text {inh }}$. Figure adapted from Auth et al., (2017).

firing rate function $\phi$ :

$$
\bar{F}_{i}=\phi\left(\bar{u}_{i}\right)=\frac{1}{1+\exp \left(\beta\left(\epsilon-\bar{u}_{i}\right)\right)} .
$$

The dynamics of the membrane potential of the inhibitory population depends on the number $N_{\text {pop }}$ of neurons in each population:

$$
\tau^{\mathrm{inh}} \frac{\mathrm{d} u^{\mathrm{inh}}}{\mathrm{d} t}=-u^{\mathrm{inh}}+w_{\mathrm{in}}^{\mathrm{inh}} N_{\text {pop }} \bar{F}_{1}+w_{\text {in }}^{\mathrm{inh}} N_{\text {pop }} \bar{F}_{2} .
$$

We use $N_{\text {pop }}=N_{\mathrm{CA}} \approx 104.3$ as obtained from the expression for the number of neurons in a CA in Equation (4.12) (compare Figure 4.5). The firing rate of the inhibitory population is given by the same firing rate function $\phi^{\text {inh }}$ as in the full network model:

$$
F^{\text {inh }}=\phi^{\text {inh }}\left(u^{\text {inh }}\right)=\frac{1}{1+\exp \left(\beta^{\text {inh }}\left(\epsilon^{\text {inh }}-u^{\text {inh }}\right)\right)} .
$$

We assume that the dynamics of the mean synaptic weights $\bar{w}_{i}^{\text {rec }}$ of the recurrent synapses within the two populations follows the same plasticity rule as an individual recurrent synaptic weight:

$$
\tau^{\mathrm{rec}} \frac{\mathrm{d} \bar{w}_{i}^{\text {rec }}}{\mathrm{d} t}=\bar{F}_{i}^{2}+\frac{F^{\mathrm{T}}-\bar{F}_{i}}{1-F^{\mathrm{T}}}\left(\bar{w}_{i}^{\mathrm{rec}}\right)^{2} .
$$

Also the mean weights of the feedforward synapses projecting the two input stimuli onto the populations are assumed to follow the same plasticity rule as the weight of a single feedforward synapse:

$$
\tau^{\mathrm{ff}} \frac{\mathrm{d} \bar{w}_{i k}^{\mathrm{ff}}}{\mathrm{d} t}=\bar{F}_{i} \bar{I}_{k}+\frac{F^{\mathrm{T}}-\bar{F}_{i}}{1-F^{\mathrm{T}}}\left(\bar{w}_{i k}^{\mathrm{ff}}\right)^{2}
$$

In summary, the dynamics of the population model are described by nine independent dynamical variables: the mean membrane potentials $\bar{u}_{1}$ and $\bar{u}_{2}$ of the two excitatory populations, the membrane potential $u^{\text {inh }}$ of the inhibitory population, the mean weights $\bar{w}_{1}^{\text {rec }}$ and $\bar{w}_{2}^{\text {rec }}$ of recurrent synapses within the two populations, and the four mean weights $\bar{w}_{1 A}^{\mathrm{ff}}, \bar{w}_{1 B}^{\mathrm{ff}}, \bar{w}_{2 A}^{\mathrm{ff}}$ and $\bar{w}_{2 B}^{\mathrm{ff}}$ of the feedforward synapses. As we 
Table 4.2: Overview of the population model parameters and their respective values.

\begin{tabular}{llr}
\hline Parameter Description & Symbol & Value \\
\hline number of neurons in each excitatory population & $N_{\text {pop }}$ & 104.3 \\
mean number of intra-population synapses per neuron & $\bar{n}_{\text {rec }}$ & 21.0 \\
mean number of synapses from active inputs per neuron & $\bar{n}_{++}^{++}$ & 13.3 \\
synaptic weight from inhibitory population to recurrent area & $w_{\text {out }}^{\text {inh }}$ & -20 \\
synaptic weight from recurrent area to inhibitory population & $w_{\text {in }}^{\text {inh }}$ & 1 \\
steepness of excitatory firing rate function & $\beta$ & 1 \\
inflection point of excitatory firing rate function & $\epsilon$ & 12 \\
steepness of inhibitory firing rate function & $\beta^{\text {inh }}$ & 1 \\
inflection point of inhibitory firing rate function & $\epsilon^{\text {inh }}$ & 100 \\
target firing rate of synaptic scaling & $F^{\top}$ & 0 \\
time constant of excitatory neurons & $\tau$ & $10 \mathrm{~ms}$ \\
time constant of inhibitory population & $\tau^{\text {inh }}$ & $10 \mathrm{~ms}$ \\
time constant of recurrent plasticity & $\tau^{\text {rec }}$ & $10 \mathrm{~s}$ \\
time constant of feedforward plasticity & $\tau^{\text {ff }}$ & $10 \mathrm{~s}$ \\
\hline
\end{tabular}

assume that at any point of time at least one of the two stimuli is inactive, always two of the four feedforward connections can be omitted. This effectively reduces the population model to a total of seven dimensions. Table 4.2 summarizes all parameters of the model.

\subsubsection{Population Nullclines and Equilibria}

In contrast to the underlying network model with its 900 neurons and several thousands of plastic synapses, the reduction to a population model with only nine or rather seven dynamical variables allows an analytical investigation of the underlying structure of the equilibria. This analysis is based on determining the intersections of the membrane potential nullclines of the two excitatory populations.

The equilibrium values $\bar{w}_{i}^{\text {rec, } *}, i \in\{1,2\}$, of the mean recurrent synaptic weights are given as a function of the equilibrium values $\bar{F}_{i}^{*}$ of the population activities from Equation (4.17):

$$
\bar{w}_{i}^{\mathrm{rec}, *}\left(\bar{F}_{i}^{*}\right)=\sqrt{\frac{1-F^{\mathrm{T}}}{\bar{F}_{i}^{*}-F^{\mathrm{T}}}} \bar{F}_{i}^{*} .
$$

Accordingly, the equilibrium values $\bar{w}_{i k}^{\mathrm{ff}, *}, i \in\{1,2\}$ and $k \in\{\mathrm{A}, \mathrm{B}\}$, of the mean weights of the feedforward synapses are obtained as a function of $\bar{F}_{i}^{*}$ from Equation (4.18):

$$
\bar{w}_{i k}^{\mathrm{ff}, *}\left(\bar{F}_{i}^{*}\right)=\sqrt{\frac{1-F^{\mathrm{T}}}{\bar{F}_{i}^{*}-F^{\mathrm{T}}} \bar{F}_{i}^{*} \bar{I}_{k}} .
$$

The only interaction between the two excitatory populations is mediated by the inhibitory population. We derive the equilibrium value $u^{\text {inh, } *}$ of its membrane potential as a function of both $\bar{F}_{1}^{*}$ and $\bar{F}_{2}^{*}$ from Equation (4.15):

$$
u^{\mathrm{inh}, *}\left(\bar{F}_{1}^{*}, \bar{F}_{2}^{*}\right)=w_{\text {in }}^{\text {inh }} N_{\text {pop }}\left(\bar{F}_{1}^{*}+\bar{F}_{2}^{*}\right) .
$$

We transform $u^{\mathrm{inh}, *}$ into the equilibrium firing rate $F^{\mathrm{inh}, *}$ by means of the firing rate function $\phi^{\text {inh }}$ of the inhibitory population (Equation 4.16):

$$
F^{\mathrm{inh}, *}\left(\bar{F}_{1}^{*}, \bar{F}_{2}^{*}\right)=\phi^{\mathrm{inh}}\left(u^{\mathrm{inh}, *}\left(\bar{F}_{1}^{*}, \bar{F}_{2}^{*}\right)\right) .
$$


In Equations (4.19), (4.20) and (4.22), we express the equilibrium values of the weights of the plastic synapses and of the inhibitory firing rate as functions of the equilibrium values of the mean firing rates of the excitatory populations. We insert these expressions into the dynamical equation of the membrane potentials of the excitatory populations (Equation 4.13). Thus, we obtain a system of equations which implicitly define the two population nullclines $(i \in\{1,2\})$ :

$$
0=-\bar{u}_{i}^{*}+\bar{n}_{++}^{\mathrm{rec}} \bar{w}_{i}^{\mathrm{rec}, *}\left(\bar{F}_{i}^{*}\right) \bar{F}_{i}^{*}+w_{\mathrm{out}}^{\mathrm{inh}} F^{\mathrm{inh}, *}\left(\bar{F}_{1}^{*}, \bar{F}_{2}^{*}\right)+\sum_{k=\mathrm{A}, \mathrm{B}} \bar{n}_{++}^{\mathrm{ff}} \bar{w}_{i k}^{\mathrm{ff}, *}\left(\bar{F}_{i}^{*}\right) \bar{I}_{k} .
$$

Using $\bar{F}_{i}^{*}=\phi\left(\bar{u}_{i}^{*}\right)$, we transform the two equations into explicit function equations describing the respective population nullclines $(i, j \in\{1,2\}$ and $i \neq j)$ :

$$
\bar{u}_{j}^{*}=\phi^{-1}\left[\frac{\phi^{\mathrm{inh},-1}\left(\frac{1}{w_{\text {out }}^{\text {in }}}\left(u_{i}-\bar{n}_{++}^{\text {rec }} \bar{w}_{i}^{\text {rec }, *}\left(\bar{u}_{i}^{*}\right) \phi\left(u_{i}^{*}\right)-\sum_{k} \bar{n}_{++}^{\mathrm{ff}} \bar{w}_{i k}^{\mathrm{ff}, *}\left(\bar{u}_{i}^{*}\right) \bar{I}_{k}\right)\right)}{w_{\text {in }}^{\text {inh }} N_{\text {pop }}}-\phi\left(u_{i}^{*}\right)\right] .
$$

The inverse firing rate functions $\phi^{-1}$ and $\phi^{\mathrm{inh},-1}$ are analytically accessible.

The nullcline $\bar{u}_{j}^{*}\left(\bar{u}_{i}^{*}\right)$ gives the mean membrane potential $\bar{u}_{j}^{*}$ of neurons in population $j$ which is necessary for an equilibrium at which the neurons in population $i$ have the mean membrane potential $\bar{u}_{i}^{*}$. Actual equilibria of the system require that both equations are fulfilled and are therefore located at intersections of the two nullclines (Figure 4.11 a). Due to the nonlinearity of the nullclines in Equation (4.24), their intersections can only be numerically determined. To do so, we use the methods provided by the SciPy software package (Jones et al., 2001). For the set of parameters which we derived from the network model (Table 4.2), the dynamics of the population model is dominated by a total of three equilibria if one of the two input stimuli is active. We determine the stability of these equilibria by evaluating the eigenvalues of the Jacobi matrix of the system (Appendix A.2) at the respective locations of the phase space. We find that there are two stable equilibria in the population model separated by an unstable one on the symmetry axis (Figure $4.11 \mathrm{a}$ ). The two stable equilibria (2, 3) represent states in which the neurons of one of the two populations have a high mean membrane potential $\bar{u}_{i}^{*}$. The mean membrane potential of the neurons in the respective other population is close to zero.

Using the firing rate equation for the two excitatory populations (Equation 4.14), we project the nullclines and equilibria of the population model into the space which is spanned by the two mean population firing rates $\bar{F}_{i}^{*}$ (Figure $4.11 \mathrm{~b}$ ). As to be expected, the asymmetric equilibria $(2,3)$ are characterized by a close to maximum firing rate in one population and almost no neuronal activity in the other population. Furthermore, Equation (4.19) allows to project the nullclines and the equilibria into the space spanned by the mean weights $\bar{w}_{1}^{\text {rec,* }}$ and $\bar{w}_{2}^{\text {rec,**}}$ of the recurrent synapses (Figure 4.11c). The high or low level of activities in the two equilibria are accompanied by corresponding strong or weak recurrent synaptic weights. The stable equilibria $(2,3)$ therefore describe the formation of a CA in one of the two populations.

It is noteworthy that there is no stable equilibrium for low or intermediate firing rates and corresponding low or intermediate recurrent weights in both populations. This illustrates the preference of the system to build up local clusters of high activity instead of unclustered and wide-spread intermediate activity and explains the reliable formation of CAs observed in the simulations of the full network model. 

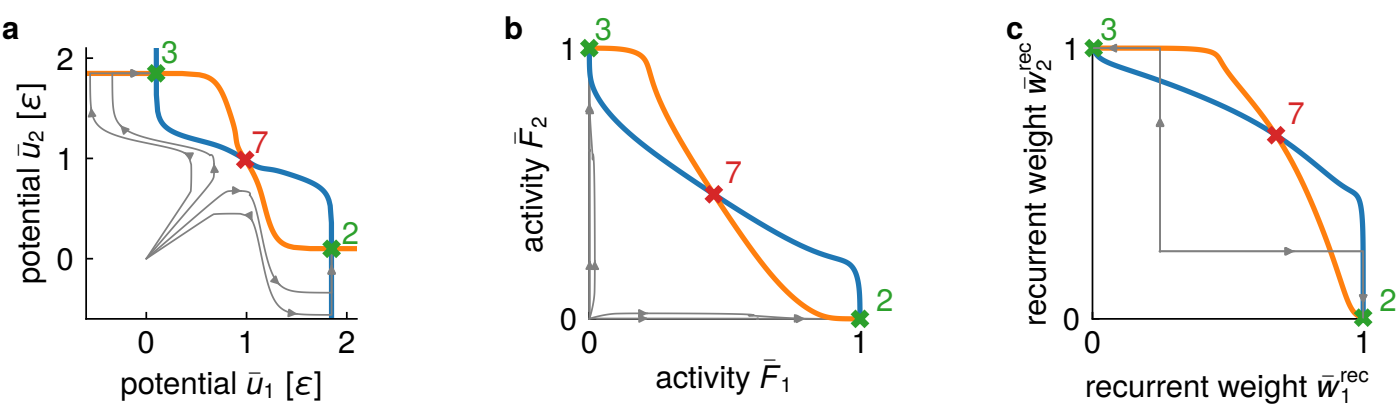

$\begin{array}{ll}- \text { population } 1 \text { nullcline } & * \text { stable equilibrium } \\ - \text { population } 2 \text { nullcline } & * \text { unstable equilibrium }\end{array}$

Figure 4.11: Nullclines and equilibria of the population model. (a) The nullcline of population $i$ is given by the mean membrane potential $\bar{u}_{j}^{*}$ in population $j \neq i$ which results in an equilibrium in population $i$ with mean membrane potential $\bar{u}_{i}^{*}$. Intersections of the two nullclines are equilibria of the system which are either asymptotically stable or unstable. The two asymmetric stable equilibria $(2,3)$ indicate the formation of a CA in population 1 or 2 , respectively. They are separated by an unstable equilibrium (7). (b) The projection of the nullclines and equilibria into the activity space shows that the formation of CAs is accompanied by a high mean firing rate in the respective population. (c) In the space spanned by the mean weights of the recurrent synapses, the stable equilibria correspond to maximum recurrent weights in one of the populations and almost zero weights in the second population. $(\mathbf{a}, \mathbf{b}, \mathbf{c})$ The numbers of the equilibria correspond to the numbers in Figure 4.12. The gray lines show example trajectories of the integrated population model for slightly different initial conditions. Figure adapted from Auth et al., (2017).

\section{Input Amplitude Bifurcation}

The nullclines of the population model and, as such, also the equilibria calculated from the population model do not only depend on the chosen parametrization of the model, but also on the properties of the input stimulus. For instance, in living neuronal systems, we cannot assume that all input signals are characterized by the same stimulus amplitude. Therefore, here, we study the bifurcation behavior of the population model for varying input amplitudes $\bar{I}_{\mathrm{A}}$. In other words, we investigate the stable and unstable equilibria of the population model as a function of $\bar{I}_{\mathrm{A}}$ (Figure 4.12).

For very low stimulus intensities $\bar{I}_{\mathrm{A}} \lesssim 0.35$, there are either two unstable equilibria $(2,3)$ representing CA formations in one of the two populations or no such equilibria at all $\left(\bar{I}_{\mathrm{A}} \lesssim 0.25\right)$. A symmetric equilibrium (1) with low recurrent weights in both populations is the only stable equilibrium of the system in this input amplitude regime. This equilibrium corresponds to no CA being formed in either of the two populations. Thus, neither can new CAs be learned nor existing ones reliably recalled. Still, this does not exclude the possibility to recall an already existing CA on short time scales by solely exploiting activity dynamics.

For stimulus intensities $0.35 \lesssim \bar{I}_{\mathrm{A}} \lesssim 0.8$, the population model still possesses the stable symmetric equilibrium (1) which corresponds to low potentials $\bar{u}_{i}$ and low recurrent weights $\bar{w}_{i}^{\text {rec }}$ in both populations. However, in this input regime, there are also the two stable asymmetric equilibria $(2,3)$ existing. These equilibria correspond to the formation of a CA in one of the two populations. They are separated from the stable symmetric equilibrium by two unstable equilibria $(5,6)$. Which of the three stable equilibria is reached depends on the initial conditions of the model. The stable symmetric equilibrium (1) is likely to be reached from intermediate and low initial recurrent weights, i.e., from a situation in which there is no CA existing in either of the two populations. The stable asymmetric equilibria $(2,3)$, in turn, are reached from 


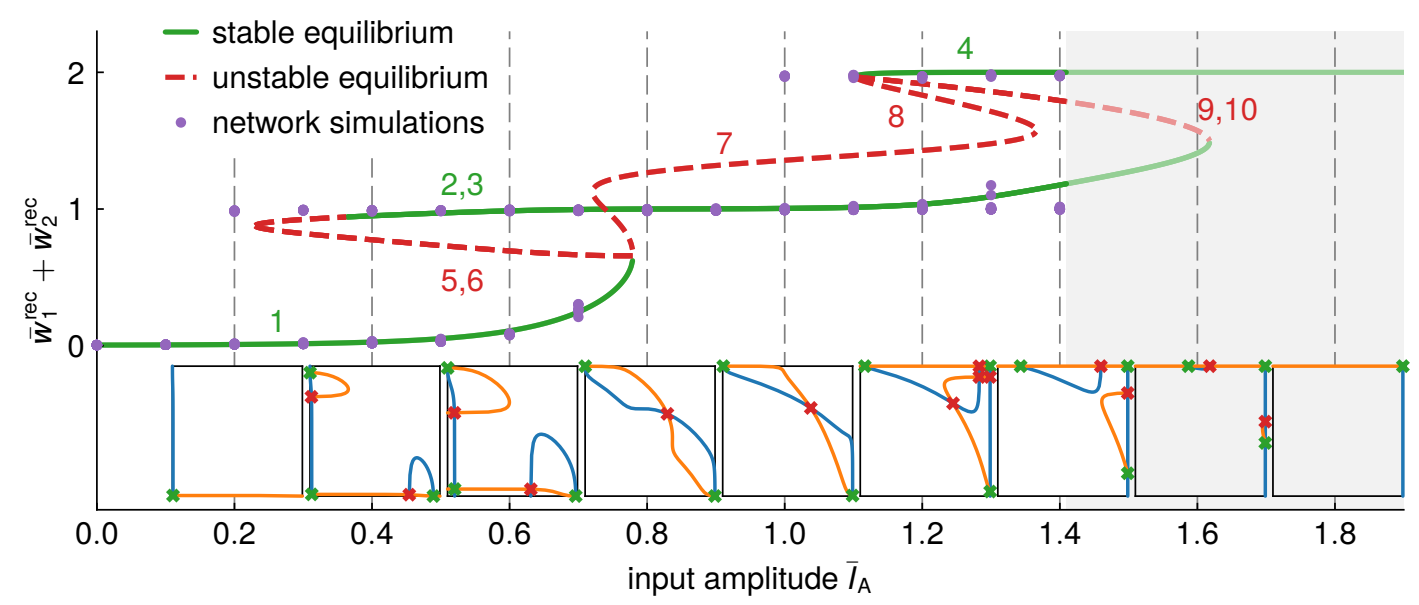

Figure 4.12: Bifurcation diagram of the population model for varied input amplitudes $\bar{I}_{\mathrm{A}}$. Shown is the sum of the mean recurrent weights $\bar{w}_{1}^{\text {rec,* }}$ and $\bar{w}_{2}^{\text {rec, } *}$ within the two populations for the respective equilibria. This projection provides a good overview over the bifurcation diagram as it allows to distinguish symmetric equilibria at different recurrent weight levels. The red and green numbers identify the different equilibria (compare Figure 4.11). Note that in some cases, two different equilibria are projected onto the same line in this bifurcation diagram. The violet points stem from long-run network simulations $\left(10^{4} \mathrm{~s}\right)$ with different initial conditions of the feedforward and recurrent weights corresponding to zero, one or two CAs in the network (10 runs each per value of $\bar{I}_{\mathrm{A}}$, see main text for details). While the network simulations show the same general bifurcation behavior, the exact positions of these bifurcations differ from the population model due to the simplifying assumptions of the latter. In the gray shaded area $\left(\bar{I}_{\mathrm{A}} \gtrsim 1.41\right)$, even maximum inhibition is not able to balance the sum of excitatory feedforward and recurrent inputs received by neurons in the recurrent area. Thus, CA growth is unbounded (Appendix A.3). In this regime, the population model does not apply. The insets at the bottom show the nullclines and the equilibria at their intersections in the $\bar{w}_{1}^{\text {rec }}-\bar{w}_{2}^{\text {rec }}$-space. Stable equilibria are marked green, unstable ones are red. Figure adapted from Auth et al., (2017).

initial conditions in which a CA is already existing in one of the two populations. Thus, input amplitudes in this regime suffice to recall existing CAs. This recall is reliable, i.e., does not reduce the respective feedforward and recurrent weights in the long run.

For higher input amplitudes $0.8 \lesssim \bar{I}_{\mathrm{A}} \lesssim 1.1$, the two stable equilibria $(2,3)$ dominate the dynamics of the model. There is no more stable symmetric equilibrium existing. In this regime, the formation of a CA in one of the two populations is robust to variations of the stimulus intensity $\bar{I}_{\mathrm{A}}$. Only if there is perfect symmetry in the initial conditions, the unstable equilibrium (7) is reached. In all other cases, a CA is formed in one of the two populations.

For even higher stimulus intensities $1.1 \lesssim \bar{I}_{\mathrm{A}} \lesssim 1.35$, an additional stable equilibrium (4) accompanied by a total of three new unstable equilibria $(8,9,10)$ emerges. The stable equilibrium (4) is characterized by a high mean membrane potential and thus also close to maximum recurrent weights in both populations. This may either correspond to epileptic activity of the complete network or to the formation of two CAs for a given stimulus. However, this equilibrium is densely "shielded" by the three unstable equilibria $(8,9,10)$ and therefore unlikely to be reached from usual initial conditions.

Even though there are stable equilibria in the population model for input amplitudes $\bar{I}_{\mathrm{A}} \gtrsim 1.41$, these do no longer relate to stable CAs in the complete network model. This is due to the fact that for these input amplitudes, the inhibition in the network model is no longer able to balance the excitatory signals in the recurrent area. Therefore, the CA growth is not limited and eventually all neurons within the 
recurrent area become active. The derivation of the maximum input amplitude for CA formation is outlined in Appendix A.3.

For the range $\bar{I}_{\mathrm{A}} \lesssim 1.41$, we compare the bifurcation curve with results obtained from simulating the complete network dynamics with stimuli of the respective input amplitudes $\bar{I}_{\mathrm{A}}$. In order to reach the different equilibria, we perform these simulations with three different initial weight configurations: In addition to the normal homogenous initialization, we also perform simulations in which we initially create one or two CAs consisting of approximately $N_{\text {pop }}$ neurons in the recurrent area by scaling up the respective recurrent and feedforward weights to their maximum values. Furthermore, in order to reach the equilibrium of the network dynamics in finite time, at the end of the simulation period $\left(10^{4} \mathrm{~s}\right)$, we set the plastic synaptic weights to their respective equilibrium values as defined by the final pre- and postsynaptic activity. For each value of $\bar{I}_{\mathrm{A}}$, we simulate ten independent network instantiations for each of the three initial weight configurations. If there are two distinct active CAs in the final state, we consider the corresponding active neurons as populations 1 and 2. If there is only one active CA in the final state, we consider all active neurons as population 1 and the circular group of $N_{\text {pop }}$ neurons with the highest topological distance to population 1 as population 2 . If there is no active CA in the final state, we define two distinct circular groups of $N_{\text {pop }}$ neurons each as populations 1 and 2. The resulting values of the sum of the mean recurrent weights within these two populations show a similar bifurcation behavior than obtained for the population model (violet points in Figure 4.12). However, for small values of $\bar{I}_{\mathrm{A}}$, differences emerge regarding the maximum input amplitude $\bar{I}_{\mathrm{A}}$ for which the formation of a CA is a stable equilibrium of the network dynamics. Furthermore, also the position of the bifurcation at $\bar{I}_{\mathrm{A}} \approx 1.1$ at which a stable equilibrium with two active CAs emerges differs between the population model and the network simulations. We assume that in both cases, this is due to the fixed population size $N_{\text {pop }}$ underlying the bifurcation in the population model. In the complete network model, smaller CAs may emerge which result in less inhibitory signals received by the neurons in the recurrent area. Thus, lower excitatory feedforward signals may still suffice to elicit the formation of a CA.

In summary, the analysis of the bifurcation curve of the population model for varying input amplitudes $\bar{I}_{\mathrm{A}}$ shows that CAs can be reliably formed for a wide range of different values $0.8 \lesssim \bar{I}_{\mathrm{A}} \lesssim 1.35$. A reliable recall of existing CAs is possible also for smaller values of $I_{\mathrm{A}} \gtrsim 0.35$.

\subsubsection{Relevance of Feedforward Plasticity}

Up to now, we concentrated our discussion of the self-organized CA formation in the population model on the dynamics of the membrane potential and the weights of the recurrent synapses within the two populations. In parallel, however, also the weights of the feedforward synapses develop according to the plasticity rule stated in Equation (4.18). While the equilibrium value of this combination of Hebbian plasticity and synaptic scaling for small target firing rates $F^{\mathrm{T}}$ mostly (in the case of $F^{\mathrm{T}}=0$ solely) depends on the presynaptic activity (Equation 4.20), the postsynaptic activity value strongly determines the time scale of the adaptation toward this equilibrium value.

In the case of the feedforward synapses in the population model, the presynaptic activity level is given by the stimulus intensity $\bar{I}_{k}, k \in\{\mathrm{A}, \mathrm{B}\}$. The postsynaptic activity level corresponds to the activity $\bar{F}_{i}, i \in\{1,2\}$, of the population in the recurrent area. Assuming $\bar{I}_{k}$ and $\bar{F}_{i}$ to be constant, the mean weight dynamics of 
the feedforward synapses described by Equation (4.18) can be exactly solved by separation of variables. In the special case $\bar{F}_{i} \cdot \bar{I}_{k}=0$, the solution is given by

$$
\bar{w}_{i k}^{\mathrm{ff}}(t)=\left(\frac{1}{\bar{w}_{i k}^{\mathrm{ff}}(0)}+\frac{\bar{F}_{i}-F^{\mathrm{T}}}{1-F^{\mathrm{T}}} \frac{t}{\tau^{\mathrm{ff}}}\right)^{-1} .
$$

If $\bar{F}_{i} \cdot \bar{I}_{k}>0$, the solution reads as follows:

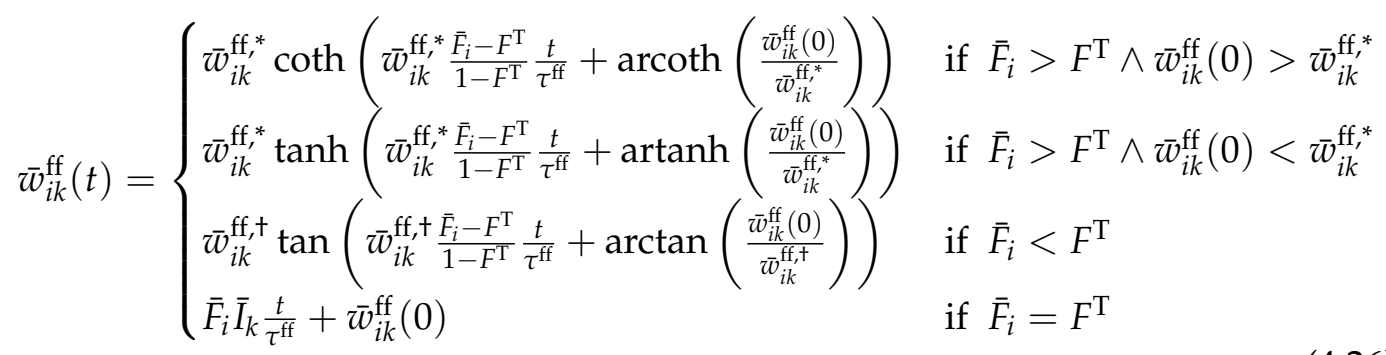

with $\bar{w}_{i k}^{\mathrm{ff}, *}=\sqrt{\bar{F}_{i} \bar{I}_{k}\left(1-F^{\mathrm{T}}\right) /\left(\bar{F}_{i}-F^{\mathrm{T}}\right)}$ and $\bar{w}_{i k}^{\mathrm{ff}, \mathrm{t}}=\sqrt{\bar{F}_{i} \bar{I}_{k}\left(1-F^{\mathrm{T}}\right) /\left(F^{\mathrm{T}}-\bar{F}_{i}\right)}$.

In all cases, the rate of the adaptation is directly scaled by either the factor $\bar{F}_{i}-F^{\mathrm{T}}$ or by $\bar{F}_{i}$. Thus, a high population activity $\bar{F}_{i}$ results in a fast evolution toward the equilibrium value $\bar{w}_{i k}^{\mathrm{ff}, *}$ defined by the stimulus intensity $\bar{I}_{k}$. When starting from an intermediate value $\bar{w}_{i j}^{\mathrm{ff}}(0)$, high values of $\bar{F}_{i}$ and $\bar{I}_{k}$ result in strong potentiation of the mean synaptic weight (case II in Figure 4.13 a). As defined in Section 2.1, this kind of plasticity which is evoked by strong simultaneous pre- and postsynaptic activity is referred to as homosynaptic potentiation. A high population activity $\bar{F}_{i}$ in combination with a low stimulus intensity $\bar{I}_{k}$, on the other hand, leads to strong and fast downscaling of the mean synaptic weight due to the low equilibrium value $\bar{w}_{i k}^{\mathrm{ff}, *}$ (case I in Figure $4.13 \mathrm{a}$ ). As this downscaling is essentially due to the postsynaptic activity evoked by signals from other neuronal inputs, it can be classified as heterosynaptic depression (compare Section 2.1). Low values of the population activity $\bar{F}_{i}$, in contrast, slow down the evolution of the synaptic weight toward the respective equilibrium value. Both homosynaptic potentiation and heterosynaptic depression result only in negligible changes of the synaptic weights for low values of $\bar{F}_{i}$ independent of the specific presynaptic input stimulus intensity $\bar{I}_{k}$ (cases III and IV in Figure 4.13 a).

All of the four discussed limiting cases of the plasticity dynamics of the feedforward synapses contribute to the formation of a CA in the recurrent area. In the following, we assume that due to a symmetry breaking condition, stimulus A is assigned to population 1 . For instance, initially, population 1 might receive slightly stronger feedforward synapses from stimulus A than population 2 . This means the system initially resides in a regime in the phase space in which the presentation of stimulus A leads to the formation of a CA in population 1 (Figure $4.13 \mathrm{~b}$, blue). We refer to this regime as the A1-regime. In contrast, the A2-regime of the phase space is given by the set of states from which on the presentation of stimulus $A$ results in a CA in population 2 (Figure $4.13 \mathrm{~b}$, orange). During the formation of the CA in population 1 , the plasticity of the recurrent synapses in population 1 leads to a strong potentiation of their mean weight $\bar{w}_{1}^{\text {rec }}$. The high activity of both presynaptic and postsynaptic activity additionally results in strong homosynaptic potentiation of the mean feedforward weight $\bar{w}_{1 \mathrm{~A}}^{\mathrm{ff}}$ (case II). Both processes drive the system away from the boundary between the A1-regime and the A2-regime implying a more stable 

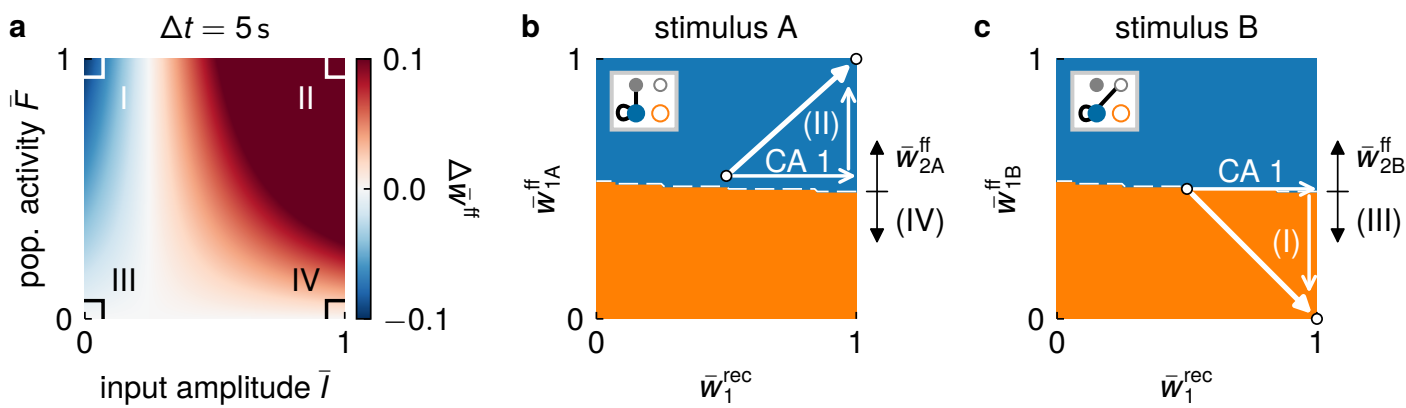

Figure 4.13: The role of heterosynaptic plasticity in the self-organized assignment of multiple CAs. (a) The change $\Delta \bar{w}^{\text {ff }}$ of the mean weight of the feedforward synapses, here in a time interval of $\Delta t=5 \mathrm{~s}$, depends strongly on both the mean population activity $\bar{F}$ and the mean input amplitude $\bar{I}$. The four small black and white rectangles (I-IV) indicate the four limiting cases of near to maximum or near to minimum activities, respectively. Note that $\Delta \bar{W}^{\text {ff }}$ vanishes in cases III and IV. (b, c) During the formation of a CA in one of the two populations (here population 1), the four different cases (I-IV) of the feedforward plasticity are exploited to pronounce the assignment of the active stimulus to this population. The blue and orange regimes in the $\bar{w}_{1}^{\text {rec }}-\bar{w}_{1 A}^{\mathrm{fH}}$-space and the $\bar{w}_{1}^{\text {rec }}-\bar{w}_{1 B}^{\mathrm{fH}}$-space indicate the phase-space regimes in which either population 1 (blue) or population 2 (orange) is recruited to represent (b) stimulus $A$ or (c) stimulus $B$. The initial feedforward weight $\bar{W}_{1 \mathrm{~A}}^{\mathrm{Hf}}$ is assumed to be marginally higher than $\bar{w}_{1 \mathrm{~B}}^{\mathrm{H}}$ such that stimulus $A$ is assigned to population 1. The growth of the recurrent synapses in this population (CA 1) in conjunction with the growth of the feedforward weights $\bar{w}_{1 \mathrm{~A}}^{\mathrm{ff}}$ drives the system deeper into the regime where stimulus $A$ is represented in population 1 (b). At the same time, the shrinkage of the weight $\bar{W}_{1 \mathrm{~B}}^{\mathrm{ff}}$ prevents stimulus $\mathrm{B}$ from also being represented by population 1 (c). Note that the shown regimes also depend on the mean synaptic weights $\bar{w}_{2 A}^{\mathrm{ff}}$ and $\bar{w}_{2 \mathrm{~B}}^{\mathrm{ff}}$. These, however, are only slightly changed during the CA formation (cases III and IV). Figure adapted from Auth et al., (2017).

memory representation. A parallel potentiation of the mean weights $\bar{w}_{2 \mathrm{~A}}^{\mathrm{ff}}$ of the feedforward synapses projecting the active stimulus onto population 2 would counteract this stabilization of the memory representation. It would shift the boundary between the A1-regime and the A2-regime in the $\bar{w}_{1}^{\text {rec }}-\bar{w}_{1 \mathrm{~A}}^{\mathrm{ff}}$ space toward higher values of $\bar{w}_{1 \mathrm{~A}}^{\mathrm{ff}}$. Due to the low postsynaptic activity, however, the rate of the corresponding adaptation is very low (case IV).

The formation of a CA in population 1 influences the initial conditions for a later presentation of stimulus B (Figure $4.13 \mathrm{c}$ ). Similar to the A1- and A2-regimes defined above, also the $\bar{w}^{\mathrm{rec}}-\bar{w}_{1 \mathrm{~B}}^{\mathrm{ff}}$-space can be subdivided into a B1-regime, in which stimulus $\mathrm{B}$ is represented by a CA in population 1, and a B2-regime, in which population 2 is recruited for stimulus $B$. Not assuming any kind of initial symmetry breaking, the system initially resides exactly at the boundary between these two regimes. Without any additional modifications, the formation of a CA in population 1 would drive the model into the B1-regime. Thus, both stimuli A and B eventually would equally be represented by population 1 impeding any discrimination ability. This situation is circumvented by the heterosynaptic depression of the feedforward synapses projecting stimulus B into population 1 during the formation of the CA in population 1 (case I). This reduction of the mean weight $\bar{w}_{1 \mathrm{~B}}^{\mathrm{ff}}$ prevents the system from ending up in the B1-regime and drives it instead into the B2-regime (Figure $4.13 \mathrm{c}$, orange). Again, this could be counterbalanced by a depression of the mean weight $\bar{w}_{2 \mathrm{~B}}^{\mathrm{ff}}$ projecting stimulus B onto population 2. It would shift the boundary between the B1-regime and the B2-regime in the $\bar{w}^{\text {rec }}-\bar{w}_{1 \mathrm{~B}}^{\mathrm{ff}}$-space toward smaller values of $w_{1 \mathrm{~B}}^{\mathrm{ff}}$. As before, this counterbalancing effect is negligible due to the low postsynaptic activity level and the associated slow plasticity of the converging feedforward synapses (case III).

Thus, indeed, all four limiting cases of the plasticity of the feedforward synapses contribute critically to the successful assignment of the CA on the one hand and to 


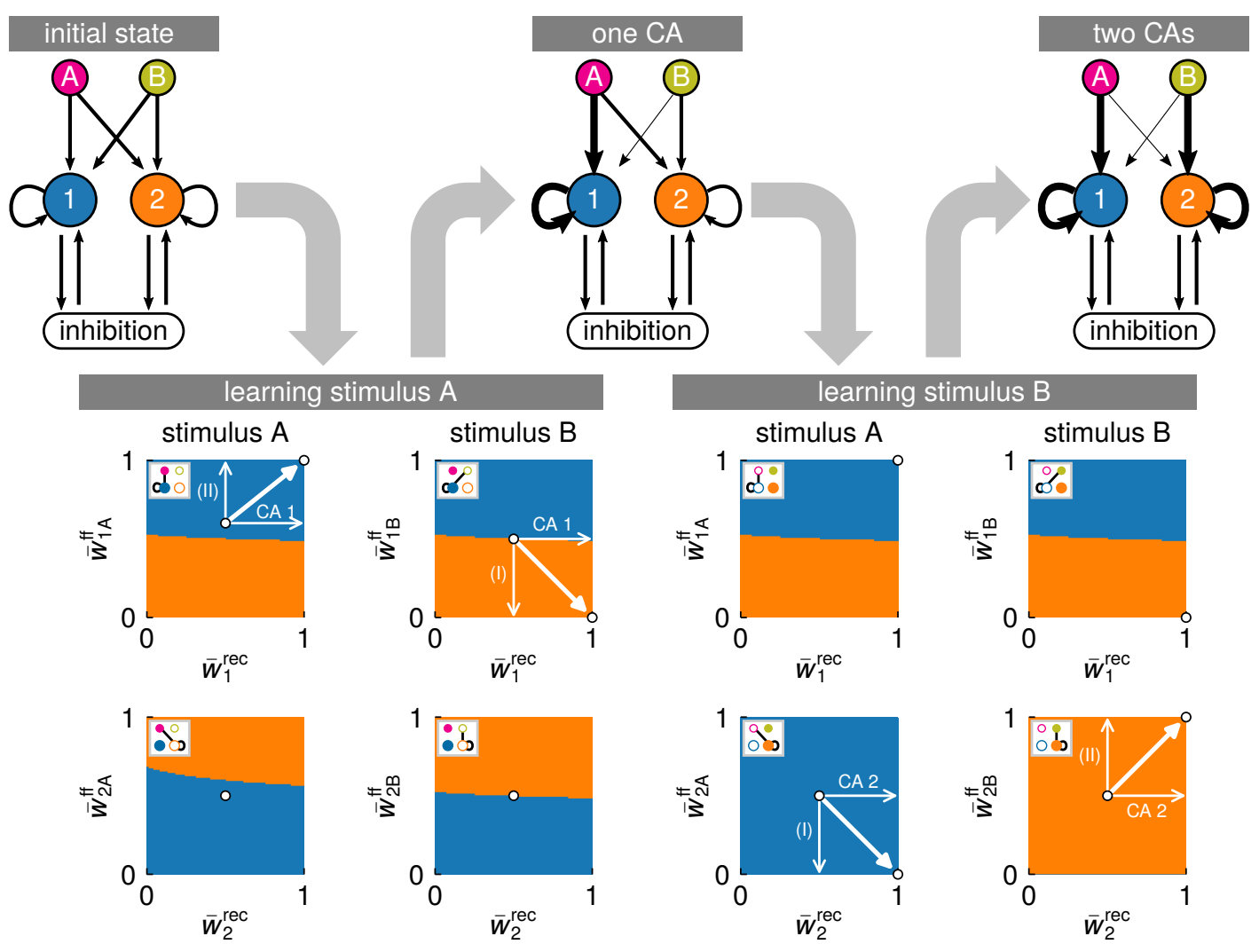

Figure 4.14: Summary of the synaptic weight changes underlying the formation and allocation of memory representations. The different figures illustrate the chronology of the assignment of the two distinct stimuli $A$ and $B$ to the two different populations 1 and 2. The sketches in the upper row illustrate the strengths of the different groups of plastic feedforward and recurrent synapses. Bolder arrows indicate stronger synaptic connections. The plots in the lower row visualize the plasticity processes during the presentation of stimulus $A$ and during the presentation of stimulus $B$, respectively, in the spaces spanned by the mean recurrent synaptic weights $\bar{w}_{1}^{\text {rec }}$ and $\bar{w}_{2}^{\text {rec }}$ and the mean feedforward synaptic weights $\bar{w}_{1 \mathrm{~A}}^{\mathrm{ff}}, \bar{w}_{1 \mathrm{~B}}^{\mathrm{ft}}, \bar{w}_{2 \mathrm{~A}}^{\mathrm{ft}}$ and $\bar{w}_{2 \mathrm{~B}}^{\mathrm{Hf}}$. As in Figures $4.13 \mathrm{~b}$ and $4.13 \mathrm{c}$, the blue area designates the regime in which the stimulus is assigned to population 1 . In the orange area, the stimulus is assigned to population 2. The shape of these regimes depends on the state of all plastic synapses in the model. The state at the beginning of the learning process is given by the white dot. The horizontal arrows labeled "CA 1" and "CA 2" show the growing of the recurrent synaptic weights. The arrows labeled (I) and (II) belong to a change of the feedforward synaptic weights according to the cases defined in Figure $4.13 \mathrm{a}$. Figure adapted from Auth et al., (2017).

the discrimination against other stimuli on the other hand. This shows that not only the structure of the equilibria but also the different time scales emerging from the underlying dynamical processes is of relevance for the self-organized formation and assignment of CAs in the network model.

\subsubsection{Summary of the Formation and Assignment of Cell Assemblies}

The study of the equilibria of the population model in the space of the recurrent weights in Section 4.3.2 and the analysis of the dynamics of the feedforward weights in Section 4.3.3 lead to a complete picture of the self-organized formation of two CAs and their assignment to two different stimuli (Figure 4.14).

Starting from a "blank" network with all synapses at their initial values, a small symmetry break decides which population (here population 1 ) represents the stimulus which is first presented to the network (here stimulus A). As a result, a CA is formed 
by potentiation of the recurrent synapses within population 1. It is furthermore assigned to the current stimulus via homosynaptic potentiation and discriminated from other stimuli by heterosynaptic depression. The reaction of the system to the presentation of a second stimulus B is biased by the results of the first CA formation. The system already resides deeply in the B2-regime, meaning that stimulus B is safely assigned to the CA which is now formed in population 2 .

The fact that even when there is not yet a CA in population 2 existing, population 2 is already designated to represent stimulus B, is an artifact of the reduction of the complete network model to a population model with only two distinct stimuli and only two different populations. If there would have been, for instance, three populations included into the model, the formation of a CA in population 1 would not influence the decision about which of the two remaining populations is going to represent stimulus B. Nevertheless, this reduction does not diminish the validity of the key observations resulting from the analyses of the population model: The successful formation of a CA requires the described equilibria structure with stable equilibria for the recruitment of a given population for the representation of a given stimulus. The correct assignment of the CA to the corresponding stimulus with parallel discrimination against other stimuli, in turn, relies on the interplay of homosynaptic potentiation, heterosynaptic depression and a slow adaptation rate of synapses with low postsynaptic activity level.

\subsection{Discussion}

In this chapter, we show that the combination of Hebbian plasticity and synaptic scaling operating on both the feedforward and the recurrent synapses in a neuronal network model enables reliable self-organized memory formation and memory allocation. The resulting CAs are input dependent attractors of the network state and can be stably stored on long time scales, i.e., implement LTM. This verifies the second sub-hypothesis of this thesis (Figure 1.1) and provides a possible mechanism by which the attractor states required for robust WM operation in tasks with unpredictable temporal structure (compare Chapter 3) may emerge in a self-organized way.

The network model which we study in this chapter is based on the model presented by Tetzlaff et al., (2013). In this study, the authors show that a CA is formed by the dynamic interaction between pure Hebbian plasticity and synaptic scaling in a predefined population of recurrently interconnected neurons. While there are several alternative models of CA formation (e.g. Litwin-Kumar and Doiron, 2014; Zenke et al., 2015; Holtmaat and Caroni, 2016), the model of Tetzlaff et al., (2013) stands out by being able to explain non-trivial effects during reconsolidation protocols (Walker et al., 2003; Tetzlaff et al., 2013). However, this model does not answer the question how different stimuli may be assigned to different neurons in the recurrent area in a self-organized way. In other words, it does not tackle the problem of memory allocation. Here, we show that including the plasticity of feedforward synapses into the model resolves this limitation.

In our model, homosynaptic potentiation of the recurrent synapses explains the formation of a CA. The correct assignment of the CA to the respective stimulus and the discrimination against other stimuli are enabled by the interplay of homosynaptic potentiation, heterosynaptic depression and slow weight adaptation for low postsynaptic activities at the feedforward synapses. While the relevance of homosynaptic potentiation and heterosynaptic depression for the formation of memory representations, or Hebbian CAs, has been pronounced before (Tetzlaff 
et al., 2013; Litwin-Kumar and Doiron, 2014; Zenke et al., 2015), these studies do not include the dynamics of the feedforward connectivity into their analyses. In contrast, other studies solely concentrate on the importance of homosynaptic potentiation and heterosynaptic depression in the feedforward connectivity to solve the memory assignment problem (Kohonen, 1982; Sullivan and Sa, 2006; Stevens et al., 2013). By considering the dynamics of both the feedforward and the recurrent synapses in parallel, the here presented network model does not only confirm the relevance of homosynaptic potentiation and heterosynaptic depression in both processes, but also reveals the relevance of a third property for the successful self-organizing of memories in neuronal systems: Low levels of postsynaptic activity have to significantly slow down the synaptic adaptation processes. This property supports the assignment and discrimination process by only potentiating synapses converging at the currently active memory representation. Experimental as well as theoretical results support the modulatory role of postsynaptic activity on the magnitude of weight changes induced by synaptic plasticity (Sjöström et al., 2001; Graupner and Brunel, 2010).

In the network model investigated in this chapter, the synapses in between excitatory neurons are subject to a synaptic plasticity rule composed of two countering terms: Hebbian plasticity and synaptic scaling. The Hebbian term is the origin of the homosynaptic potentiation, synaptic scaling produces the heterosynaptic depression. Thus, in our model, synaptic scaling contributes essentially to the successful discrimination of different stimulus representations. This goes beyond the traditionally assumed role of synaptic scaling as pure stabilization of Hebbian plasticity (Turrigiano et al., 1998; Abbott and Nelson, 2000; Turrigiano and Nelson, 2004).

A characteristic feature of the CAs emerging in the network model is their total distinctness, i.e., the fact that two CAs are not sharing any common neurons. The corresponding finding in other models of memory formation are diverse with some models also producing non-overlapping representations (e.g. Garagnani et al., 2009) and others being able to build up CAs with some neurons being shared between multiple CAs (e.g. Litwin-Kumar and Doiron, 2014). Theoretically, it is known that overlapping assemblies provide a higher storage capacity than distinct ones (Tsodyks and Feigel'man, 1988). Additionally, the overlap between different LTM representations has been proposed to underly the sequential recall of information from LTM (Katkov et al., 2017). Experimentally, neuronal representations of different sounds in the auditory cortex have been shown to indeed posses overlaps (Bathellier et al., 2012). In the here presented network model, the distinctness of the CAs arises due to the competition introduced by the synaptic scaling term, i.e., due to heterosynaptic downscaling of the weights of synapses transmitting signals from inactive neurons to highly active neurons. Thus, a modification of the used plasticity rule would be necessary to allow for the formation of overlapping CAs in this model. For instance, adding a dependence on the presynaptic activity to the synaptic scaling term might provide a possible solution (Liu, 2011).

As long as a new stimulus is able to recall an earlier learned CA in the recurrent area, no new CA can emerge in our network model. The transition from pattern separation to pattern completion for varying stimulus similarities and ongoing synaptic plasticity (Figure $4.7 \mathrm{~b}$ ) gives the maximum stimulus similarity between a new stimulus and an already learned one which allows for the generation of a new CA. Given the parametrization which we used in this chapter, this maximum similarity is comparably small. Thus, our network model is not able to discriminate stimulus patterns with a significant overlap. It is an open question how this discrimination ability may be increased. A possible solution might be given by intrinsic plasticity (Zhang and Linden, 2003) processes at the neurons in the recurrent area. Intrinsic 
plasticity refers to the activity-dependent persistent modification of the properties of a neuron. This intrinsic plasticity might, for instance, increase the parameter $\epsilon$ of the firing rate function of neurons which are recruited for a CA. This would lead to a higher sum of incoming excitatory signals being required to activate neurons within a CA and would therefore increase the specificity of this CA. Alternatively, inhibitory regulation mechanisms (Isaacson and Scanziani, 2011) or inhibitory plasticity mechanism (Vogels et al., 2011; Kullmann et al., 2012) might also allow for stronger stimulus discrimination abilities.

Anatomically, declarative LTM is assumed to be implemented in the hippocampus and in the neocortex (Eichenbaum, 2000; Nadel and Hardt, 2011). The recurrent area of the network model which we study in this chapter contains 900 neurons. This is of course still orders of magnitudes smaller than the number of neurons in the human cortex (Herculano-Houzel, 2009) or the human hippocampus (West and Gundersen, 1990). When scaling up our model, several assumptions underlying the architecture of the network model will have to be adapted. While it is known that in small cortical areas, inhibitory neurons indeed interact with a high fraction of the excitatory neurons (Fino and Yuste, 2011; Isaacson and Scanziani, 2011), the assumption of an unspecific global inhibition does not hold on larger cortical distances (Muir and Cook, 2014). Thus, it remains an open question how effective competition between different neuronal populations about representing a given stimulus may be realized in large networks with a realistic topology.

The CAs which are formed in our network model are input dependent attractor states of the network dynamics. Thus, in order to stay active, they require ongoing excitatory feedforward signals. Importantly, these signals do not have to be of the same intensity as the signal required to form a new CA (Figure 4.12). When interacting with the WM system, a low level feedforward activity might be provided by attention gating mechanisms as proposed by Cowan, (1988). Alternatively, lowering the level of global inhibition would allow a CA to maintain persistent activity also in the absence of excitatory feedforward signals. In both cases, the activity of a specific CA (a specific attractor state) would provide the information of past inputs as required by the WM system to operate on tasks with unpredictable temporal structure. 



\section{Chapter 5}

\section{Fast Adaptation of Central Pattern Generators}

In Chapter 3, we show that the neuronal system implementing working memory (WM) requires both transient dynamics and distinct attractor states to operate on tasks with unpredictable temporal structure. We demonstrate that in the employed formulation of the n-back task, the duration during which the neuronal system moves along the transient trajectory in between two different attractor states suffices to produce the required target signal. Indeed, also humans performing an n-back task typically have to produce rather short response actions whenever a new stimulus arrives. In more realistic settings, however, based on processes in $\mathrm{WM}$, a subject might have to produce or adapt motor control signals whose durations are much longer than the duration of a transient trajectory in the neuronal WM system. For instance, it may be required to control the movement of writing, speaking or walking. Thus, the question arises how such long lasting control signals may be produced based on short lasting transient signals arriving from WM.

Many natural motor control tasks rely on periodic movements. In particular, this includes the different types of locomotion like walking, running or swimming. As discussed in Section 2.5.1, these periodic movements are controlled by neuronal signals which are produced by specific structures called central pattern generators (CPGs; Hooper, 2001; Ijspeert, 2008). CPGs are neuronal circuits which are able to produce periodic neuronal signals without the need for any periodic input. Thus, these systems are dominated by periodic attractor states resulting in ongoing periodic activity. If a subject needs to activate such a periodic signal or to adapt its properties based on processes in WM, this requires WM to be able to interact with the respective CPG and to influence, for instance, the frequency of the produced signal. As an example, we may imagine a subject who is told to walk at some given velocity but only after having received a specific "Go"-signal. In this task, first, the subject has to keep the information about the specified velocity in its WM. Second, when receiving the "Go"-signal, the subject has to use this information to appropriately tune the respective CPGs. If this tuning process depends on ongoing active control by WM, this would require a long transient trajectory within the neuronal system implementing WM. This would prevent the storing of new information and the performance of further manipulations and computations. In contrast, the third subhypothesis of this thesis (Figure 1.1) states that transient signals can fast and precisely adapt the frequency of periodic attractors of CPGs in a self-organized way. If this subhypothesis holds, WM could be released from active control after a short adaptation time. In order to verify this sub-hypothesis, we need to find a mechanism which allows a short-lasting signal to rapidly adapt the output frequency of a CPG.

Mathematically, a CPG can be described as a nonlinear system whose dynamics possesses a stable limit cycle, i.e., a periodic attractor. Such a system is referred to as 
a nonlinear oscillator. The adaptive frequency oscillator (AFO) mechanism allows to adapt the frequency of nonlinear oscillators based on an external signal originating, for instance, from WM (Section 5.1). Oscillators adapted by the AFO mechanism actually learn the frequency of the external signal and are able to keep oscillating at this frequency even after the removal of the external signal. In order to be a plausible candidate mechanism of the WM-CPG interaction, however, the adaptation provided by the AFO mechanism has to be fast and precise. Here, we show that the AFO mechanism does not provide the ability for fast and precise adaptations, in particular when considering a larger range of possible external frequencies (Section 5.2). Therefore, we develop and analyze a new mechanism which overcomes this deficit (Section 5.3). This new mechanism relies on dynamically adjusting the coupling strengths between the external signal and the nonlinear oscillator. We refer to this mechanism as "Adaptation through Fast Dynamical Coupling" (AFDC). Compared to the AFO mechanism, the AFDC mechanism allows significantly faster and more precise adaptations within a wider range of frequencies (Section 5.3.4). We discuss the implications of these results for our third sub-hypothesis as well as in the context of biological plausibility and possible applications of the mechanism for robotic control problems (Section 5.4)

Most of the results presented in this section have been published in the following journal article:

T. Nachstedt, C. Tetzlaff, and P. Manoonpong (2017). Fast dynamical coupling enhances frequency adaptation of oscillators for robotic locomotion control.

Frontiers in Neurorobotics 11, pp. 1-14. DOI: 10.3389/fnbot.2017.00014.

\subsection{Methods}

In this section, first, we define the general concept of an oscillator as used in this thesis. Furthermore, we introduce two specific realizations of oscillators which are used throughout this chapter. We continue by summarizing the adaptive frequency oscillator mechanism and by applying this mechanism to the two previously introduced oscillators. Finally, we introduce a set of measures which we use to quantitatively evaluate the quality of a given frequency adaptation process of an oscillator.

\subsubsection{Oscillators}

In mathematical terms, an oscillator is defined as an autonomous dynamical system with at least one limit cycle attractor, i.e., a stable two-dimensional periodic orbit (Buchli et al., 2006b). The projection of such a system on two variables $x$ and $y$ which span the plane of the limit cycle can be expressed as a system of two general dynamical equations:

$$
\begin{aligned}
& \dot{x}(t)=f_{x}(x(t), y(t), \theta) \\
& \dot{y}(t)=f_{y}(x(t), y(t), \theta) .
\end{aligned}
$$

The functions $f_{x}$ and $f_{y}$ define the dynamics of the system on the limit cycle. We explicitly include the dependency on a parameter $\theta$ which determines the intrinsic oscillation frequency $v$ of the system. In many cases, there is no analytic expression of the relation $v(\theta)$ between the frequency $v$ and the parameter $\theta$ available. In the following, we only assume that this function is monotone. Apart from this, $v(\theta)$ may be an arbitrary function. 


\section{Hopf Oscillator}

The Hopf oscillator (Andronov et al., 1971) is described by the following system of dynamical equations for the state variables $x$ and $y$ :

$$
\begin{aligned}
& \dot{x}(t)=\left(\mu-r(t)^{2}\right) x(t)-\theta y(t) \\
& \dot{y}(t)=\left(\mu-r(t)^{2}\right) y(t)+\theta x(t)
\end{aligned}
$$

with $r(t)=\sqrt{x(t)^{2}+y(t)^{2}}$. The dynamics of the system is dominated by a single asymptotically stable and harmonic limit cycle with radius $\mu>0$ and an angular frequency of exactly $\theta$. Accordingly we have $v(\theta)=\theta /(2 \pi)$.

\section{Van der Pol Oscillator}

The second oscillatory system which we investigate in this chapter is the Van der Pol oscillator (Van der Pol, 1920). Without external stimulation, the Van der Pol oscillator is described by the following system of equations for the state variables $x$ and $y$ :

$$
\begin{aligned}
& \dot{x}(t)=y(t) \\
& \dot{y}(t)=\mu\left(1-x(t)^{2}\right) y(t)-\theta^{2} x(t) .
\end{aligned}
$$

Here, the parameter $\mu \geq 0$ determines the degree of nonlinearity of the limit cycle which is harmonic for $\mu=0$. The variable $\theta$ determines the frequency $v$ of the oscillations. In contrast to the Hopf oscillator, there is no analytical expression for the function $v(\theta)$ available. We approximate this function numerically (see Appendix B for details).

\subsubsection{Adaptive Frequency Oscillators}

Based on the general concept of an oscillator, the original adaptive frequency oscillator (AFO) mechanism has been proposed by Righetti et al., (2006). It describes a mechanism that can be universally applied to any kind of nonlinear oscillatory system where a frequency-determining variable $\theta$ is available. The method is able to adapt the intrinsic frequency of the oscillator to the frequency of an external periodic stimulation.

Transforming the general oscillator in Equation (5.1) into an oscillator with AFO mechanism requires two modifications. In a first step, the variable $x$ of the oscillator is coupled to an external periodic stimulation $F(t)$ via a coupling constant $\epsilon$. The value of $\epsilon$ determines the strength of the influence of the external stimulation on the oscillator:

$$
\begin{aligned}
& \dot{x}(t)=f_{x}(x(t), y(t), \theta(t))+\epsilon F(t) \\
& \dot{y}(t)=f_{y}(x(t), y(t), \theta(t)) .
\end{aligned}
$$

In a second step, the frequency parameter $\theta$ is turned into a dynamic variable $\theta(t)$ which is adapted according to the following dynamics (Righetti et al., 2006):

$$
\dot{\theta}(t)= \pm \eta F(t) \frac{y(t)}{\sqrt{x(t)^{2}+y(t)^{2}}} .
$$

The learning rate $\eta$ determines the speed of the adaptation process. The sign of the right-hand side of Equation (5.5) depends on the orientation of the oscillations in the phase space of the specific oscillator at hand. In the original formulation, 
always $\epsilon=\eta$ is chosen. This choice is suggested by the derivation the adaptation rule (Equation 5.5) from the effect of the stimulation $F(t)$ on the phase velocity of an oscillator (Righetti et al., 2006). In general, however, there is no a priori reason for choosing $\epsilon=\eta$ to obtain optimal adaption behavior.

In conjunction, Equations (5.4) and (5.5) describe how to transform an oscillator as defined by Equation (5.1) into an AFO. This mechanism has been successfully applied to a wide range of different oscillatory systems including harmonic and nonharmonic ones (Righetti et al., 2006). Additionally, it has been shown that the AFO mechanism enables adaptation of the intrinsic frequency to very different external stimuli (Righetti et al., 2006). In the following, we discuss the application of the AFO mechanism to the purely harmonic Hopf oscillator (Equation 5.2) and to the Van der Pol oscillator (Equation 5.3) with a highly non-harmonic limit cycle.

\section{Hopf Oscillator with AFO Mechanism}

As a first example, the AFO mechanism is applied to the harmonic Hopf oscillator. Following the schema described above, we couple the variable $x(t)$ to an external stimulation $F(t)$. Additionally, we turn the frequency parameter $\theta$ into a dynamic variable $\theta(t)$ which is adapted according to Equation (5.5). This results in the following system of equations for the Hopf oscillator with AFO mechanism:

$$
\begin{aligned}
& \dot{x}(t)=\left(\mu-r(t)^{2}\right) x(t)-\theta(t) y(t)+\epsilon F(t) \\
& \dot{y}(t)=\left(\mu-r(t)^{2}\right) y(t)+\theta(t) x(t) \\
& \dot{\theta}(t)=-\eta F(t) \frac{y(t)}{\sqrt{x(t)^{2}+y(t)^{2}}} .
\end{aligned}
$$

We couple this system to a periodic stimulation $F(t)$ with a frequency $v_{\text {ext }}$. Importantly, $v_{\text {ext }}$ is different from the initial intrinsic frequency $v_{0}$ of the oscillator as determined by the initial value $\theta_{0}$ of $\theta(t)$. This results in the typical AFO adaptation curve (Figure 5.1 a). As the frequency adaptation is based on the correlation between $F(t)$ and $y(t)$, the intrinsic frequency of the oscillator is not modified as long as $F(t)=0$ holds $(t<100$ in Figure 5.1 a). As soon as we switch $F(t)$ on, $\theta(t)$ starts to slowly develop into the direction of the value $\theta_{\text {ext }}(100<t<600)$. Here $\theta_{\text {ext }}$ denotes the value of the frequency variable $\theta(t)$ which corresponds to the frequency $v_{\text {ext }}$ in the unperturbed system. The adaptation of $\theta(t)$ gets faster the closer $\theta(t)$ gets to $\theta_{\text {ext }}$. Briefly before $\theta(t)$ converges to a quasi-constant state with only small periodic fluctuations, a brief overshoot is observed. The small fluctuations in the quasi-constant state are due to the ongoing adaption which averages to zero over one oscillation period $(600<t<700)$. Once the quasi-constant state is reached, the external stimulation $F(t)$ can be removed. The oscillator continues to oscillate with the learned frequency $(t>700)$. 
a

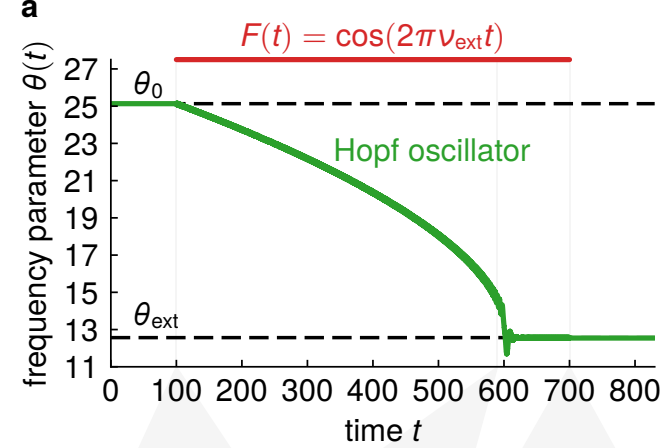

$$
-x(t) \quad-y(t) \quad-F(t)
$$

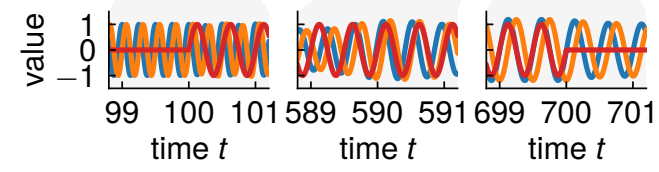

b

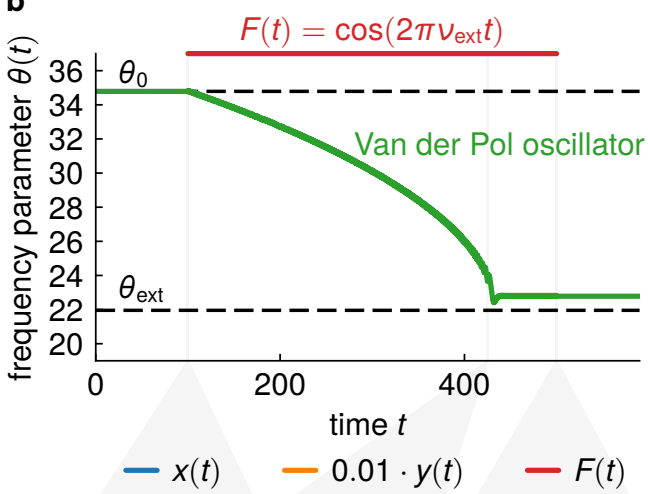

$99 \cos _{\text {time } t}^{100101424} 425426499500501$
time $t$

Figure 5.1: Adaptation of two oscillators with AFO mechanism. A Hopf oscillator with AFO mechanism (a) and a Van der Pol oscillator with AFO mechanism (b) adapt their intrinsic frequency to a sine-wave stimulation $F(t)$ with a frequency $\nu_{\text {ext }}=2.0$ and unit amplitude. The initial intrinsic frequency of both oscillators is $\nu_{0}=4.0$. The time interval during which the stimulation is applied is indicated by the red bar. The values $\theta_{0}$ and $\theta_{\text {ext }}$ (horizontal dashed line) of the frequency variable correspond to the exact frequencies $\nu_{0}$ and $\nu_{\text {ext }}$ of the unperturbed oscillators. The small insets at the bottom show the oscillating variables $x$ and $y$ of the oscillator and the signal $F(t)$ at the time of the onset of the stimulation, shortly before convergence and at the end of the stimulation. (a) The Hopf oscillator with AFO mechanism and parameter values $\mu=1.0$ and $\epsilon=\eta=1.0$ adjusts its intrinsic frequency to a value very close to $\nu_{\text {ext }}$. The values of $\theta(t)$ which correspond to the frequencies $\nu_{0}$ and $\nu_{\text {ext }}$ are $\theta_{0}=2 \pi \nu_{0} \approx 25.1$ and $\theta_{\mathrm{ext}}=2 \pi \nu_{\mathrm{ext}} \approx 12.6$. (b) The quasi-converged state of the Van der Pol oscillator with AFO mechanism and parameter values $\mu=100.0$ and $\epsilon=\eta=0.7$ is characterized by an offset of the final intrinsic frequency from $\nu_{\text {ext }}$. The relevant values of $\theta(t)$ have been numerically determined to be $\theta_{0} \approx 34.8$ and $\theta_{\text {ext }} \approx 22.0$ (Appendix B). Figure adapted from Nachstedt et al., (2017).

\section{Van der Pol Oscillator with AFO Mechanism}

Based on the regular Van der Pol oscillator in Equation (5.3), the Van der Pol oscillator with AFO mechanism is given by the following set of equations:

$$
\begin{aligned}
& \dot{x}(t)=y(t)+\epsilon F(t) \\
& \dot{y}(t)=\mu\left(1-x(t)^{2}\right) y(t)-\theta(t)^{2} x \\
& \dot{\theta}(t)=+\eta F(t) \frac{y(t)}{\sqrt{x(t)^{2}+y(t)^{2}}} .
\end{aligned}
$$

When the Van der Pol oscillator with AFO mechanism is coupled to an external periodic stimulation with a frequency $v_{\text {ext }}$ which is different from the initial intrinsic frequency $v_{0}$ of the oscillator, the behavior of the frequency variable $\theta(t)$ resembles the one discussed for the Hopf oscillator with AFO mechanism (Figure $5.1 \mathrm{~b}$ ). However, for the Van der Pol oscillator with AFO mechanism, it is more likely to end up in a quasi-constant state in which the final value of the frequency variable $\theta(t)$ is significantly different from the value $\theta_{\text {ext }}$ which would correspond to the frequency of the external stimulation $v_{\mathrm{ext}}$. As a result, the Van der Pol oscillator with AFO mechanism will oscillate at a slightly different frequency once the external stimulation is switched of. As we discuss later in this chapter, this phenomenon is not restricted to the Van der Pol oscillator and can only be reduced at the cost of longer convergence 
times.

\subsubsection{Adaptation Quality Indicators}

The trade-off between fast adaptation times and precise adaptation results can be quantitatively captured by introducing three measures characterizing the respective features of a given adaptation process (Figure 5.2).

As long as the external stimulation $F(t)$ is active, the adaptation rule in Equation (5.5) results in ongoing changes of the frequency parameter $\theta(t)$. However, at some point, the system reaches a quasi-converged state in which the changes of $\theta(t)$ integrate to zero over one oscillation period. In other words, in this state, the mean value $\bar{\theta}$ calculated over one oscillation period is constant. The duration until the quasi-convergence is reached is captured by the convergence time $\Delta$. We define $\Delta$ as the time interval between the onset of the external stimulation and the last deviation of the frequency parameter $\theta(t)$ of the system of more than $5 \%(10 \%$ for the Van der Pol oscillator) from the finally reached mean value $\bar{\theta}$. In general, $\Delta$ should be small to allow fast reconfiguration of the oscillatory output signal.

The precision of the adaptation process is captured by two measures reflecting orthogonal properties of the intrinsic frequency in the quasi-converged state. First, the difference between the mean value $\bar{\theta}$ of the frequency-determining variable of the oscillator and the value $\theta_{\text {ext }}$ should be small for a precise adaptation. Therefore, we define the frequency offset $\delta_{\theta}=\theta_{\text {ext }}-\bar{\theta}$. Remember that $\theta_{\text {ext }}$ is defined as the value of $\theta(t)$ at which the intrinsic frequency of the oscillator corresponds to the frequency $v_{\text {ext }}$ of the external simulation. Second, the amplitude of the fluctuations of $\theta(t)$ around its mean value $\bar{\theta}$ should be small in the quasi-converged state. In case of large amplitudes of these fluctuations, the frequency stored by the oscillator once the external stimulation vanishes would strongly depend on the exact point of time at which the external stimulation is removed. (see $F(t)=0$ in Figure 5.2). This reduces the reliability of the stored frequency. The magnitude of the fluctuations in the quasiconverged state is captured by the standard deviation $\sigma_{\theta}$ of the frequency-determining variable $\theta(t)$.

For interpreting the three defined quality measures, we assume that the frequency $v_{\text {ext }}$ of the external stimulation defines the relevant time scale of a given adaptation process. Therefore, we need to set the convergence time $\Delta$, the frequency offset $\delta_{\theta}$ and the standard deviation $\sigma_{\theta}$ in relation to $v_{\text {ext }}$. Equivalently, we can set them in relation to the corresponding value $\theta_{\text {ext }}$ of the frequency-determining variable. We introduce three relative measures scaled by the cycle duration $v_{\text {ext }}^{-1}$ of the external stimulation or the parameter value $\theta_{\text {ext }}$ :

$$
\tilde{\Delta}=\frac{\Delta}{v_{\mathrm{ext}}^{-1}}, \quad \tilde{\delta}_{\theta}=\frac{\delta_{\theta}}{\theta_{\mathrm{ext}}}, \quad \tilde{\sigma}_{\theta}=\frac{\sigma_{\theta}}{\theta_{\mathrm{ext}}} .
$$

Additionally, we define a quality index $Q$ with $0 \leq Q<1$ which combines the three relative measures into a single scalar value and simplifies the evaluation of the overall quality of a given adaptation process:

$$
Q=\max \left(1-\frac{\tilde{\Delta}}{\tilde{\Delta}_{\max }}-\frac{\left|\tilde{\delta}_{\theta}\right|}{\tilde{\delta}_{\theta, \max }}-\frac{\tilde{\sigma}_{\theta}}{\tilde{\sigma}_{\theta, \max }}, 0\right) .
$$

Here, $\tilde{\Delta}_{\max }, \tilde{\delta}_{\theta, \max }$ and $\tilde{\sigma}_{\theta, \max }$ are defined as the maximum values of the respective quality measures which we allow for a reasonably good adaptation process. If either of these maximum values is reached, the combined quality measure $Q$ vanishes. 


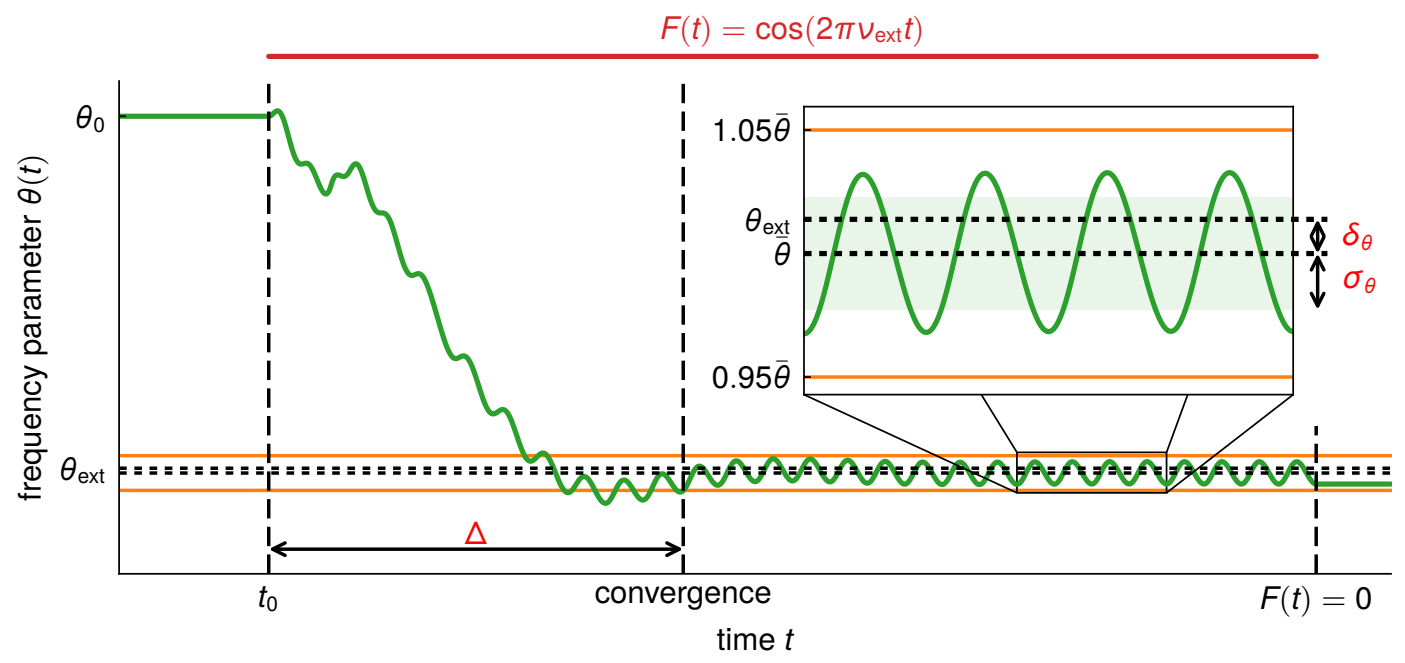

Figure 5.2: Quantitative measures of the quality of a frequency adaptation process. An AFO with an initial intrinsic frequency $\nu_{0}$, as determined by an initial frequency parameter $\theta_{0}$, is exposed to an external stimulation with a frequency $\nu_{\text {ext }}$ which corresponds to a value $\theta_{\text {ext }}$ of the frequency parameter. The stimulation sets in at time $t_{0}$ as indicated by the red horizontal bar. The inset plot in the top right shows a close up of the indicated area in the main plot. Three measures are introduced to quantify the different qualities of the frequency adaptation process. The convergence time $\Delta$ is defined as the time interval between the onset of the stimulation and the last deviation of the parameter variable $\theta(t)$ of the oscillator of more than $5 \%$ (orange horizontal lines) from the finally reached mean value $\bar{\theta}$. The difference between $\bar{\theta}$ and the value $\theta_{\text {ext }}$ which would correspond to the exact frequency $\nu_{\text {ext }}$ of the stimulation defines the frequency offset $\delta_{\theta}$. The standard deviation $\sigma_{\theta}$ of the parameter $\theta(t)$ in the converged state captures the magnitude of the final fluctuations of $\theta(t)$ (light green area). Note that after the switch-off of the external stimulation $(F(t)=0)$, the frequency parameter $\theta(t)$ remains at a value which is different from both $\theta_{\text {ext }}$ and $\bar{\theta}$. The shown time course stems from an adaptation process of an Hopf oscillator with AFO mechanism and parameter values $\mu=1.0, \epsilon=5.0, \eta=5.0$, and $\nu_{0}=2.0$. The oscillator adapts its intrinsic frequency to an external sine-wave signal with unit amplitude and frequency $\nu_{\text {ext }}=1$.0. Figure adapted from Nachstedt et al., (2017).

Alternatively, $Q$ may also be zero if the sum of the ratios in Equation (5.9) is larger than one.

In the following, we use $\tilde{\Delta}_{\max }=100, \tilde{\delta}_{\theta, \max }=0.05$, and $\tilde{\sigma}_{\theta, \max }=0.05$ when analyzing adaptation processes of the Hopf oscillator. The limit cycle of the Van der Pol oscillator is more complex than the one of the Hopf oscillator. This complicates the usage of correlation based adaptation process as employed in the AFO mechanism. Therefore, we choose different maximum values for the Van der Pol oscillator. In particular, we use $\tilde{\Delta}_{\max }=200, \tilde{\delta}_{\theta, \max }=0.1$, and $\tilde{\sigma}_{\theta, \max }=0.05$.

The three defined measures $\tilde{\Delta}, \tilde{\delta}_{\theta}$ and $\tilde{\sigma}_{\theta}$ in conjunction with the combined quality index $Q$ allow the quantitative analysis of the different adaptation processes and the study of the influence of the different adaptation parameters.

\subsubsection{Numerical Integration}

All dynamical systems in this chapter are integrated using the odeint method of the scipy python package (Jones et al., 2001). This method employs the LSODA algorithm (Brown and Hindmarsh, 1989) which is part of odepack FORTRAN library (Hindmarsh, 1983) and operates by dynamically adapting the step size of the numerical integration. 
a

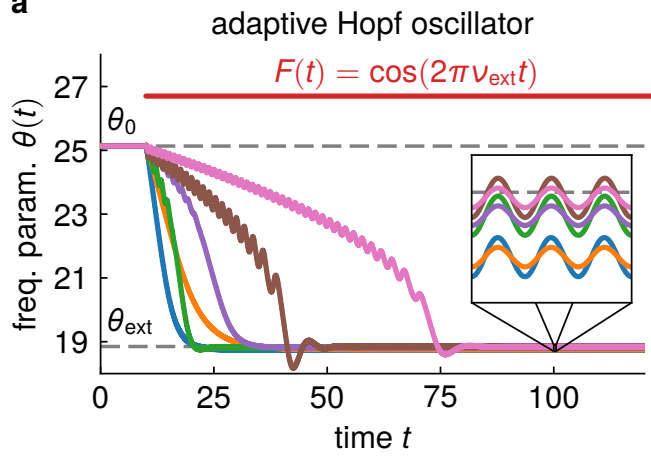

b

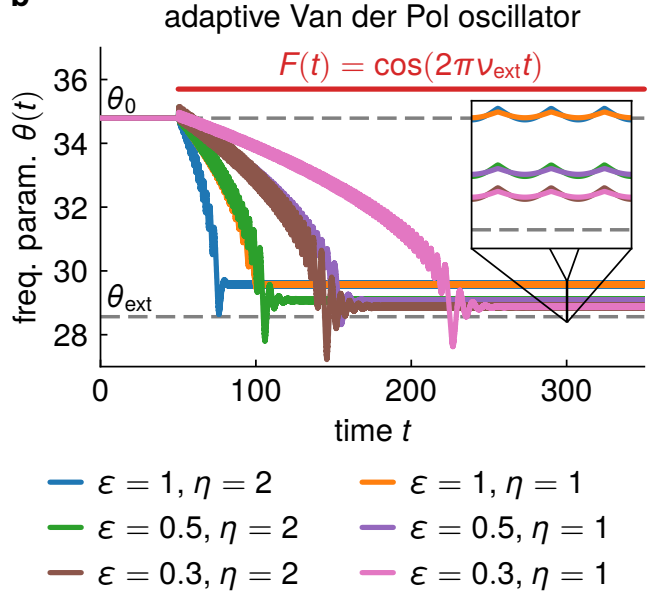

Figure 5.3: Influence of the coupling strength $\epsilon$ and the learning rate $\eta$ on the adaptation of oscillators with AFO mechanism. A higher coupling strength $\epsilon$ and a higher learning rate $\eta$ lead to shorter convergence times $\Delta$. At the same time, larger values of $\epsilon$ also result in a larger offset $\delta_{\theta}$ of the final mean parameter value $\bar{\theta}$ from the value $\theta_{\text {ext }}$. Larger values of $\eta$, in turn, lead to larger fluctuations of the frequency variable $\theta(t)$ in the quasi-converged state as measured by $\sigma_{\theta}$. Shown is the time course of the frequency parameter $\theta(t)$ for different $(\epsilon, \eta)$-parameter pairs. The time during which the external stimulation $F$ with frequency $\nu_{\text {ext }}=3.0$ is active is indicated by the red bar on the time axis. The value of the frequency parameter of the oscillator at which its internal frequency corresponds exactly to $\nu_{\text {ext }}$ is designated by $\theta_{\text {ext }}$. The initial internal frequency of the oscillator is $\nu_{0}=4.0$ as determined by the frequency parameter value $\theta_{0}$. The small insets show a close up of the time course of $\theta(t)$ in the quasi-converged state. (a) The Hopf oscillator with AFO mechanism and parameter $\mu=1.0$ can be tuned to achieve short convergence times $\Delta$ with only small values of $\delta_{\theta}$ and $\sigma_{\theta}$. (b) For the Van der Pol oscillator with AFO mechanism and parameter $\mu=100.0$, larger values of $\delta_{\theta}$ and $\sigma_{\theta}$ occur for even longer convergence times $\Delta$ than achieved in the Hopf oscillator with AFO mechanism. Figure adapted from Nachstedt et al., (2017).

\subsection{Deficits of the AFO Mechanism}

The AFO mechanism has been shown to be applicable for a variety of different oscillators and is able to adapt them to periodic signals of different shapes (Righetti et al., 2006). A common deficit of the different implementations and applications of the AFO mechanism, however, is related to the long time interval required until the internal frequency of the oscillator reaches an approximately stable value close to the frequency of the external periodic stimulation, i.e., their long convergence time. In the following, we first investigate in how far the parameters of the AFO mechanism can be tuned to accelerate the adaptation process. Afterwards, we test whether a given parameter configuration is able to provide fast adaptations for a wider range of initial and external frequencies.

\subsubsection{Trade-off between Speed and Precision}

The convergence time $\Delta$ of oscillators with AFO mechanism depends on both the coupling strength $\epsilon$ of the external stimulation to the oscillatory system and the learning rate $\eta$ of the frequency parameter $\theta(t)$. On the one hand, increasing either of the two values reduces the time interval $\Delta$ until the adaptation mechanism converges to a quasi-stable value (Figure 5.3). On the other hand, increasing $\epsilon$ or $\eta$ also results in a stronger influence of the external stimulation on the oscillatory system in general. A stronger coupling $\epsilon$ leads to a higher offset $\delta_{\theta}$ of the finally reached internal frequency from the exact frequency of the external signal (Figure 5.3). A higher learning rate $\eta$, 

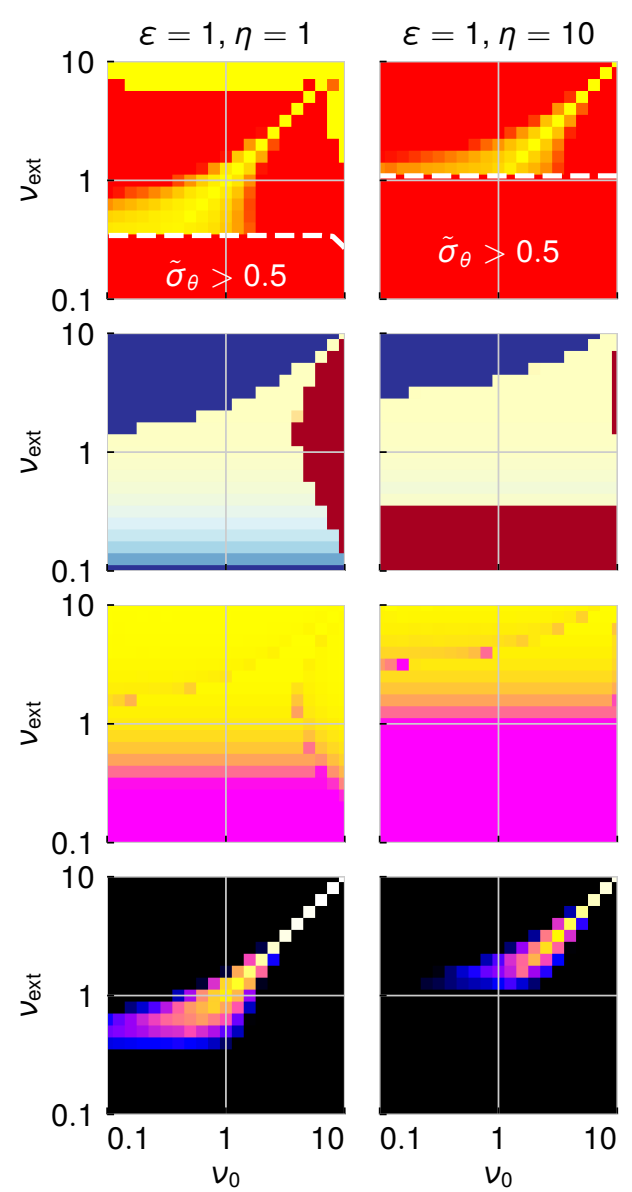
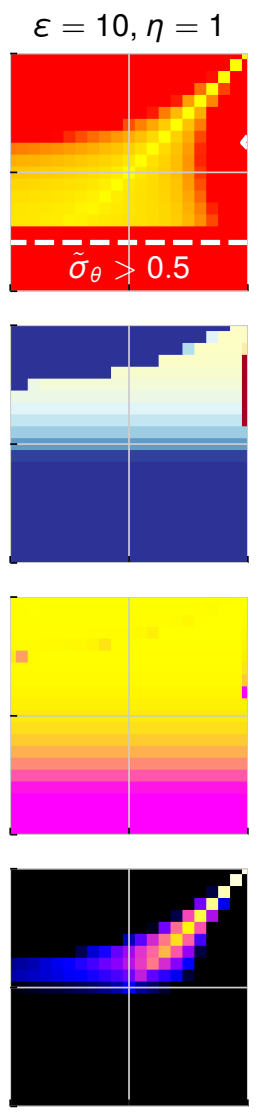

0.1
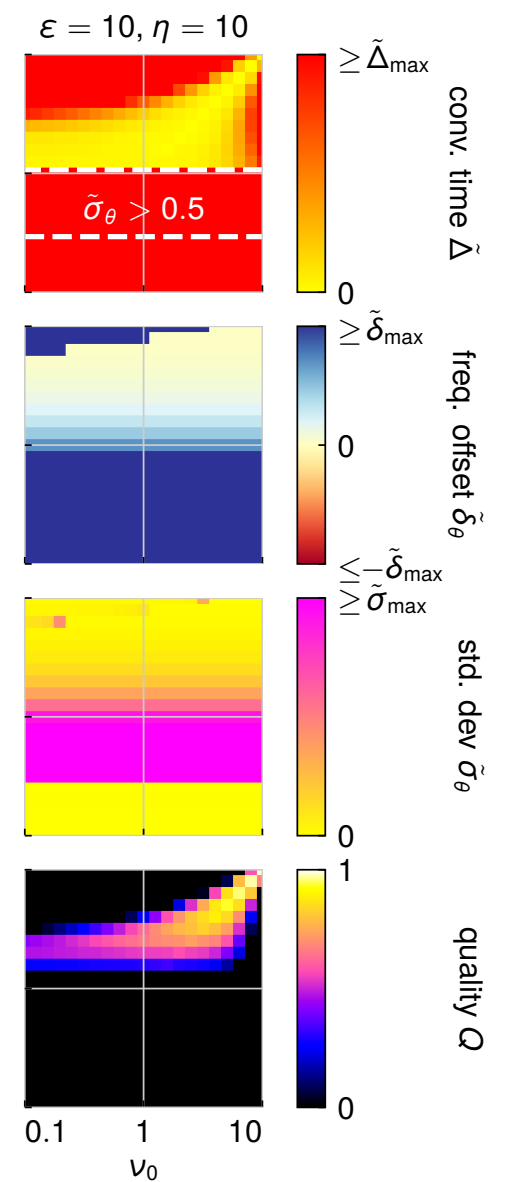

Figure 5.4: Adaptation quality measures in the $\nu_{0}-\nu_{\text {ext }}$-frequency space for different parameter values of the Hopf oscillator with AFO mechanism. For every given $(\epsilon, \eta)$-parameter pair, the respective column shows (from top to bottom) the relative convergence time $\tilde{\Delta}$, the frequency parameter offset $\tilde{\delta}_{\theta}$, the final standard deviation $\tilde{\sigma}$ and the combined quality index $Q$ in the parameter space spanned by the initial frequency $\nu_{0}$ and the external frequency $\nu_{\text {ext. }}$. For all $(\epsilon, \eta)$-parameter pairs, the area in which the adaptive Hopf oscillator allows adaptation processes with a nonzero quality index $Q$ is only a small part of the complete tested $\nu_{0}-\nu_{\text {ext }}$-frequency space. Note that the convergence time $\Delta$ is defined as the time interval between the onset of the external stimulation and the last deviation of the frequency parameter $\theta(t)$ of more than $5 \%$ from the value $\theta_{\text {ext }}$. As a consequence, $\Delta$ cannot be reasonably determined for high values of $\tilde{\sigma}_{\theta}$, i.e., takes very high values, even for $\nu_{0}=\nu_{\text {ext }}$ (indicated areas). Figure adapted from Nachstedt et al., (2017).

in turn, increases the fluctuations $\sigma_{\theta}$ of the internal frequency around the frequency of the external signal in the quasi converged state. Thus, in any case, shorter AFO convergence times $\Delta$ imply a loss of precision of the adaptation process.

\subsubsection{Adaptations Within a Wide Frequency Range}

Humans and animals expose a wide range of different behaviors. The associated movements and manipulations are performed on very different timescales. Accordingly, adaptive oscillators which are supposed to model CPG networks need to be able to adapt their intrinsic dynamics to a wide range of external frequencies. At the same time, all these adaptation processes need to be fast and precise. This can be measured in terms of the relative measures and the quality index defined in Section 5.1.3. 

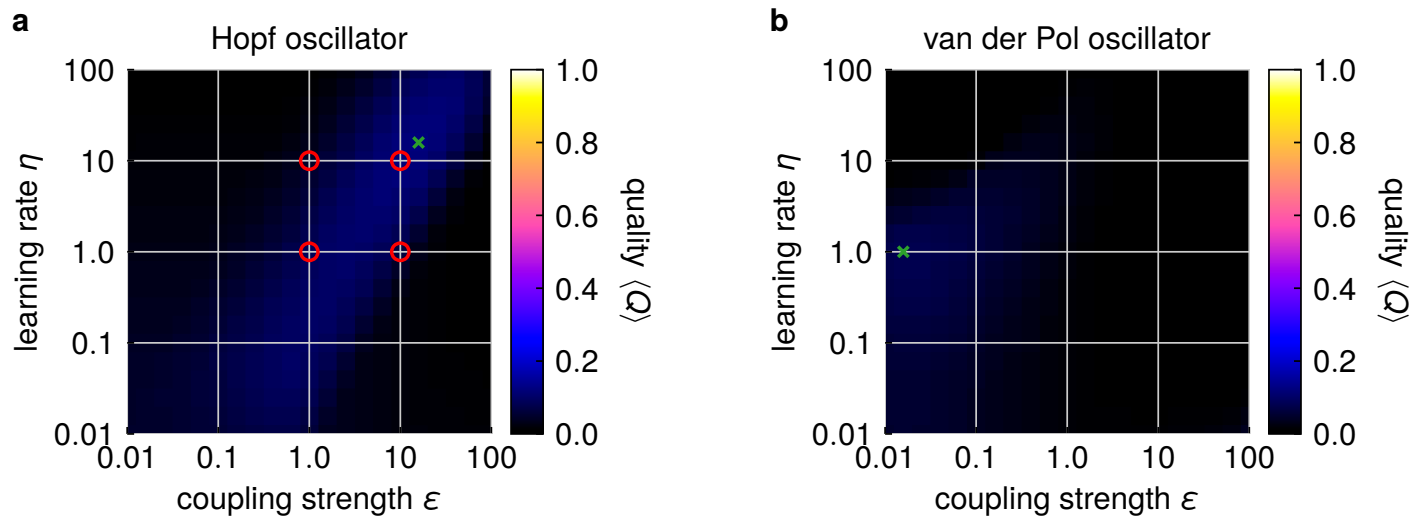

Figure 5.5: Average combined quality measure $\langle Q\rangle$ in the $\epsilon-\eta$-parameter space. For every $(\epsilon, \eta)$ parameter pair of the coupling strength $\epsilon$ and the learning rate $\eta$, the plots show the average adaptation quality index $\langle Q\rangle$ in the logarithmically sampled $\nu_{0}-\nu_{\text {ext }}$-frequency space spanned by the initial intrinsic frequency $\nu_{0}$ and the frequencies $\nu_{\text {ext }}$ of the external stimulation (compare Figure 5.4). The green crosses indicate the respective maximum values of $\langle Q\rangle$. (a) The best found configuration (green cross) of the Hopf oscillator with AFO mechanism and parameter $\mu=1.0$ allows a frequency spaced averaged quality index $\langle Q\rangle \approx 0.12$. The red circles mark the four examples shown in Figure 5.4. (b) For the Van der Pol oscillator with AFO mechanism and parameter $\mu=100.0$, the optimal configuration allows an average quality $\langle Q\rangle \approx 0.08$ (green cross). Note that we use different values of $\tilde{\Delta}_{\max }, \tilde{\delta}_{\theta, \max }$ and $\tilde{\sigma}_{\theta, \max }$ for the two different oscillators. Figure (a) is adapted from Nachstedt et al., (2017).

In the following, we analyze adaptation processes in between frequencies from two orders of magnitude of the initial intrinsic frequency $v_{0}$ of the adaptive oscillator and of the external frequency $v_{\text {ext }}$ of the stimulation: $0.1 \leq\left\{v_{0}, v_{\text {ext }}\right\} \leq 10$. Due to its harmonic limit cycle, we can tune the Hopf oscillator with AFO mechanism for shorter convergence times without the same loss of precision as observed for the Van der Pol oscillator (compare Figures $5.3 \mathrm{a}$ and $5.3 \mathrm{~b}$ ). Still, the frequency range within which the AFO mechanism allows fast as well as precise adaptation of the Hopf oscillator for a given $\epsilon-\eta$-parameter pair is very limited (Figure 5.4). Strong couplings $\epsilon$ of the external stimulation to the oscillatory system and high learning rates $\eta$ are required for large frequency intervals in which adaptation processes with fast convergence times $\tilde{\Delta}$ are possible (first row in Figure 5.4). At the same time, however, small frequency offsets $\tilde{\delta}_{\theta}$ require small values of $\epsilon$ (second row in Figure 5.4). Small standard deviations $\tilde{\sigma}_{\theta}$ of the intrinsic frequency in the converged state for a broad range of frequencies are only obtained for small values of $\eta$ (third row in Figure 5.4). The $(\epsilon, \eta)$-parameter pairs which allow a reasonable balance of speed and precision for a given frequency configuration can be identified by a nonzero value of the quality index $Q$ (bottom row in Figure 5.4). The area in the $v_{0}-v_{\text {ext }}$-space within which nonzero values of $Q$ are achieved depends strongly on the chosen parameter values. In each case, this area captures only a small portion of the complete $v_{0}-v_{\text {ext }}$-space under investigation. Thus, the trade-off between speed and precision can only be resolved for very narrow and well defined frequency configurations.

We can express the inability of the AFO mechanism to allow fast and precise adaptation of the Hopf oscillator by determining the average quality index $\langle Q\rangle$ as a function of the $(\epsilon, \eta)$-parameter pair in the logarithmically sampled frequency space defined by $0.1 \leq\left\{v_{0}, v_{\text {ext }}\right\} \leq 10$ (Figure 5.5 a). As it turns out, no $(\epsilon, \eta)$-combination allows for a value of $\langle Q\rangle$ higher than approximately 0.12 . The identical analysis of the Van der Pol oscillator results in a maximum average value of $\langle Q\rangle \approx 0.08$ (Figure $5.5 \mathrm{~b})$. Note that the maximum relative quality measures $\tilde{\Delta}_{\max }, \tilde{\delta}_{\theta, \max }$ and $\tilde{\sigma}_{\theta, \max }$ used to determine the quality index $Q$ differ between the Hopf oscillator and the Van der 
Pol oscillator (Section 5.1.3).

We conclude that in both cases, the Hopf oscillator and the Van der Pol oscillator, the AFO mechanism is not able to provide fast as well as precise adaptation over the complete range of analyzed frequencies. As a consequence, we cannot use the AFO mechanism to reliably convert short-lasting transient signals into a long-lasting periodic signal. This renders this mechanism unlikely to mediate the interaction between WM and CPGs as discussed above. In the following, we develop an extension of the AFO mechanism which is able to overcome this limitation.

\subsection{Adaptation Through Fast Dynamical Coupling}

As shown in the previous section, using the AFO mechanism, there is no fixed pair of coupling strength $\epsilon$ and learning rate $\eta$ which allows for both fast as well as precise adaptation within the investigated range of initial intrinsic frequencies and external stimulation frequencies. In general, fast adaptation tends to require high values of both $\epsilon$ and $\eta$ while precision is achieved for low values of these parameters. This frequency-specific trade-off between speed and precision does complicate applications in robotic and artificial systems. In addition, it prevents this mechanism from being a plausible model of the interaction of short-lasting transient dynamics and long-lasting periodic signals. For this interaction, it is required that the system which produces the long-lasting periodic signals is able to adapt its output fast and precisely to the inputs received from the transient system.

Here, we propose a mechanism that dynamically adjusts the coupling of the external stimulation to the oscillator during the adaptation process. This mechanism is able to achieve both fast and precise adaptation over a wide range of frequencies.

\subsubsection{Derivation of the Mechanism}

In contrast to the AFO mechanism, we do not couple the oscillatory system (Equation 5.1) directly to the external stimulation $F(t)$. Instead, we introduce a filtered signal $P(t)$ and couple this signal to the oscillator:

$$
\begin{aligned}
& \dot{x}(t)=f_{x}(x(t), y(t), \theta(t))+P(t) \\
& \dot{y}(t)=f_{y}(x(t), y(t), \theta(t)) .
\end{aligned}
$$

Accordingly, also the adaptation of the frequency variable $\theta(t)$ now depends on $P(t)$ :

$$
\dot{\theta}(t)= \pm \eta P(t) \frac{y(t)}{\sqrt{x^{2}(t)+y^{2}(t)}} .
$$

The filtered signal $P(t)$ is given by a weighted difference of the external stimulation $F(t)$ and the variable $x(t)$ of the oscillatory system:

$$
P(t)=\epsilon(t) F(t)-\beta(t) x(t) .
$$

The dynamical coupling strengths $\epsilon(t)$ and $\beta(t)$ determine the magnitude of $P(t)$. These dynamical coupling strengths are the key components of the proposed mechanism. As we have seen in Section 5.2, a strong coupling of the external stimulation leads to fast adaptation. Thus, $\epsilon(t)$ and $\beta(t)$ have to amplify the influence of the external stimulation $F(t)$ on the oscillator at the begin of an adaptation process. In contrast, a precise adaptation of the intrinsic frequency of the oscillator is enabled 
by a loose coupling of the external stimulation to the oscillator (compare Section 5.2). Therefore, the influence of $F(t)$ needs to be reduced toward the end of an adaptation process.

As we will show in the following, both of these two requirements are met by adapting $\beta(t)$ and $\epsilon(t)$ based on a combination of a correlation-based growth process and a passive decay toward a low resting value. In particular, the dynamical coupling strength $\beta(t)$ follows the following dynamics:

$$
\tau \dot{\beta}(t)=\beta_{0}-\beta(t)+\kappa P(t) x(t)
$$

with a time constant $\tau$, a correlation learning rate $\kappa$ and a decay value $\beta_{0}$. The coupling strength $\beta(t)$ scales the negative influence of the oscillator variable $x(t)$ on the filtered stimulation $P(t)$ in Equation (5.12). Its dynamics is based on the correlation of $P(t)$ and $x(t)$. This correlation is high if the oscillator is able to follow the frequency of the external stimulation which, in turn, indicates that the difference between $\theta(t)$ and $\theta_{\text {ext }}$ is low. At this stage, the adaptation process should rather concentrate on precision than on speed. Accordingly, the amplitude of $P(t)$ should be decreased. This is achieved by increasing $\beta(t)$.

The dynamical coupling strength $\epsilon(t)$ is adapted according to a very similar dynamics:

$$
\tau \dot{\epsilon}(t)=\epsilon_{0}-\epsilon(t)+\kappa F(t) P(t) .
$$

The time constant $\tau$ and the correlation learning rate $\kappa$ are the same as in Equation (5.13). The decay value $\epsilon_{0}$ may be different from $\beta_{0}$. As $\epsilon(t)$ determines the strength of the influence of the external stimulation $F(t)$ on the filtered signal $P(t)$, it is adapted based on the correlation of these two signals. If this correlation is high, the subtraction of $x(t)$ in Equation (5.12) is not able to cancel the additive contributions of $F(t)$. In other words, the difference between $\theta(t)$ and $\theta_{\text {ext }}$ is large. In this situation, an increase of $\epsilon(t)$ is desirable to increase the influence of the external stimulation on the oscillator and thereby to accelerate the adaptation process.

In conclusion, the proposed mechanism is based on automatically detecting the current phase of the adaptation process and on modifying the dynamical coupling strengths accordingly. The coupling strength $\epsilon(t)$ detects the onset of an external stimulation with a frequency different from the intrinsic one. The dynamical coupling strength $\beta(t)$, in turn, gets activated once the intrinsic frequency is close to the external one. Based on this information about the current phase of the adaptation process, the mechanism determines whether a stronger or a weaker coupling of the external stimulation to the oscillatory system is currently required. In the following, we call this mechanism "Adaptation through Fast Dynamical Coupling" (AFDC).

\subsubsection{Examples of Adaptations with the AFDC mechanism}

The AFDC mechanism can be easily applied to both the Hopf oscillator (Figure 5.6 a) and the Van der Pol oscillator (Figure 5.6 b). The Hopf oscillator equipped with the 
AFDC mechanism is given by the following system of differential equations:

$$
\begin{aligned}
\dot{x}(t) & =\left(\mu-r(t)^{2}\right) x(t)-\theta(t) y(t)+P(t) \\
\dot{y}(t) & =\left(\mu-r(t)^{2}\right) y(t)+\theta(t) x(t) \\
\tau \dot{\beta}(t) & =\beta_{0}-\beta(t)+\kappa P(t) x(t) \\
\tau \dot{\epsilon}(t) & =\epsilon_{0}-\epsilon(t)+\kappa F(t) P(t) \\
\dot{\theta}(t) & =-\eta P(t) \frac{y(t)}{\sqrt{x(t)^{2}+y(t)^{2}}}
\end{aligned}
$$

with $P(t)=\epsilon(t) F(t)-\beta(t) x(t)$.

The Van der Pol oscillator with AFDC mechanism is described by the following system of equations:

$$
\begin{aligned}
\dot{x}(t) & =y(t)+P(t) \\
\dot{y}(t) & =\mu\left(1-x(t)^{2}\right) y(t)-\theta(t)^{2} x \\
\tau \dot{\beta}(t) & =\beta_{0}-\beta(t)+\kappa P(t) x(t) \\
\tau \dot{\epsilon}(t) & =\epsilon_{0}-\epsilon(t)+\kappa F(t) P(t) \\
\dot{\theta}(t) & =+\eta P(t) \frac{y(t)}{\sqrt{x(t)^{2}+y(t)^{2}}}
\end{aligned}
$$

with $P(t)=\epsilon(t) F(t)-\beta(t) x(t)$.

A typical adaptation process of the AFDC mechanism (Figure 5.6) can be separated into several phases which we describe in the following.

As long as the external stimulation is absent $(F(t)=0)$, the dynamics of the coupling strength $\epsilon(t)$ in Equation (5.14) is solely dominated by the decay term. Therefore, $\epsilon(t)$ converges toward its resting state equilibrium $\epsilon_{0}(t<5$ in Figure 5.6 a and Figure 5.6b). As for $F(t)=0$ we have $P(t)=-\beta(t) x(t)$, the dynamics of $\beta(t)$ in Equation (5.13) involves a quadratic dependence on $x(t)$. From this equation, we obtain the equilibrium of $\beta(t)$ for $F(t)=0$ as $\beta_{0} /\left(1+\kappa \bar{x}^{2}\right)$. Here, $\bar{x}^{2}$ is the mean over time of the squared signal $x(t)^{2}$. In the absence of an external stimulation, $\beta(t)$ decays toward this value.

When the external stimulation sets in, the average product between the signal $F(t)$ and the filtered signal $P(t)$ is positive (Equation 5.12) and the value of $\epsilon(t)$ starts to increase (Equation 5.14). With larger values of $\epsilon(t)$, the average product between $F(t)$ and $P(t)$ increases. This positive feedback loop enables a fast increase of the amplitude of $P(t)$ at the begin of the adaptation process. As explained above, this accelerates the adaptation process by amplifying the influence of the stimulation on the oscillator (Equation 5.10) and on the dynamics of $\theta(t)$ (Equation 5.11).

At some point, the strong influence of the external stimulation and the resulting fast learning rate suffice to make the oscillator follow the external frequency $(t \approx 15$ in Figure $5.6 \mathrm{a}$ and $t \approx 30$ in Figure 5.6b). In other words, now, the average product of the filtered signal $P(t)$ and the oscillator variable $x(t)$ is positive and results in an increase of $\beta(t)$ (Equation 5.13). Higher values of $\beta(t)$ decrease the amplitude of $P(t)$ if $x(t)$ and $P(t)$ are correlated (Equation 5.12). A lower amplitude of $P(t)$ leads to a reduction of the average product between $P(t)$ and $x(t)$. This provides a negative feedback loop which breaks the positive feedback loop between $\epsilon(t)$ and the average product of $P(t)$ and $F(t)$ as described above (Equation 5.14). This, finally, results in a decay of both $\beta(t)$ and $\epsilon(t)$ to their respective resting values. Once the two coupling strengths are sufficiently close to these resting values, the external stimulation can be 
a
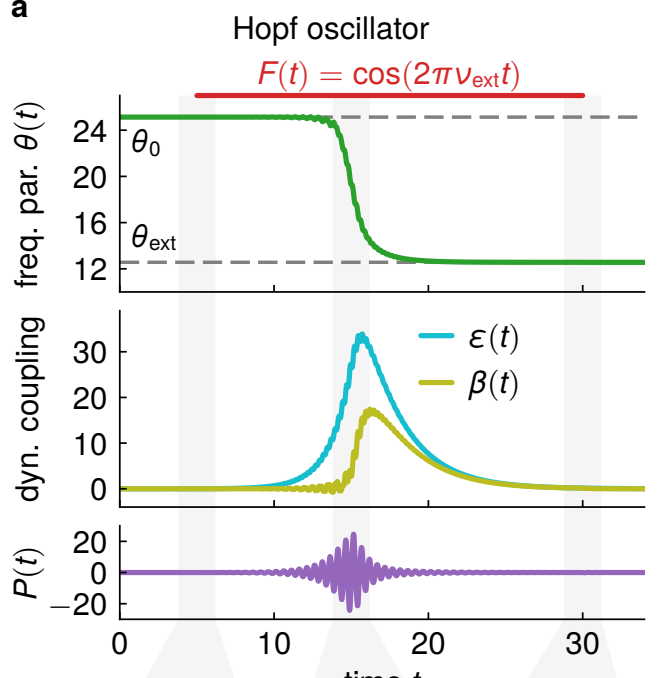

$-x(t)-y(t)-F(t)$

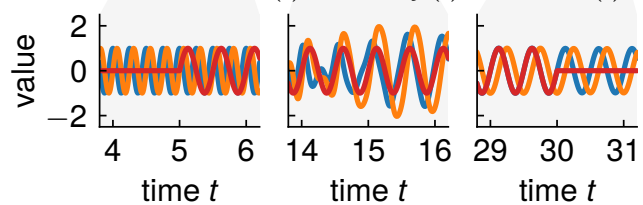

b

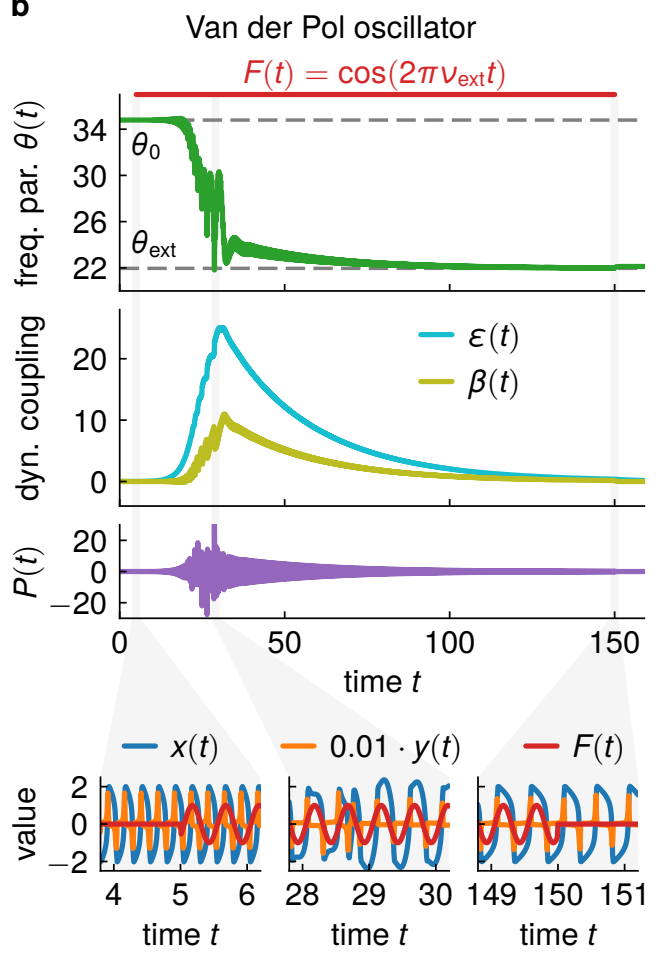

Figure 5.6: Examples of frequency adaptation with the AFDC mechanism. Coupling an oscillator with AFDC mechanism and intrinsic frequency $\nu_{0}$ to an external stimulation with a frequency $\nu_{\text {ext }}$ results in an increase of the dynamical coupling strength $\epsilon(t)$. This increases the magnitude of the signal $P(t)$ which leads to a fast adaptation of the frequency parameter $\theta(t)$ toward the value $\theta_{\text {ext }}$ which corresponds to the frequency $\nu_{\text {ext }}$. Once $\theta(t)$ is close to $\theta_{\text {ext }}$, the dynamical coupling strength $\beta(t)$ starts to grow which results in a decrease of the magnitude of $P(t)$. This allows precise adaptation of $\theta(t)$. Finally, $\epsilon(t)$ and $\beta(t)$ decay toward their respective resting values. The insets at the bottom show the variables $x(t)$ and $y(t)$ of the oscillator and the stimulation $F(t)$ at different short time-windows during the adaptation process (time intervals indicated by gray area). (a) The parameter values of the Hopf oscillator with AFDC mechanism are $\mu=1.0, \eta=0.5, K=5.0, \tau=2.0, \beta_{0}=0.0$, and $\epsilon_{0}=0.01$. The initial intrinsic frequency is $\nu_{0}=4.0$ and the frequency of the external stimulation is $\nu_{\mathrm{ext}}=2.0$. The corresponding values of the frequency variable $\theta(t)$ are $\theta_{0}=2 \pi \nu_{0} \approx 25.1$ and $\theta_{\text {ext }}=2 \pi \nu_{\text {ext }} \approx 12.6$. The external stimulation is applied for $5 \leq t<30$. (b) The parameter values of the Van der Pol oscillator with AFDC mechanism are $\mu=100.0, \eta=2.0, K=5.0, \tau=15.0, \beta_{0}=0.0$ and $\epsilon_{0}=0.01$. The frequencies $\nu_{0}$ and $\nu_{\text {ext }}$ are the same as in (a) and translate into $\theta_{0} \approx 34.8$ and $\theta_{\text {ext }} \approx 22.0$ (see Appendix B). The external stimulation is applied for $5 \leq t \leq 150$. Figure adapted from Nachstedt et al., (2017).

switched off without significantly influencing the system dynamics. The influence of the stimulation on the system has already been reduced to a minimum.

In conclusion, the combination of the dynamics of the two coupling strengths $\epsilon(t)$ and $\beta(t)$ amplifies the external stimulation as long as strong adaptation of the frequency variable $\theta(t)$ is required and reduces its magnitude toward the end of the adaptation process. This allows for a fast as well as precise adaptation of the intrinsic frequency of the oscillator.

\subsubsection{Optimal AFDC Parameters}

The AFDC mechanism has three key parameters: the time scale $\tau$ of the adaptive coupling strengths, the correlation learning rate $\kappa$, and the learning rate $\eta$ of the frequency variable $\theta(t)$. Technically, the decay values $\epsilon_{0}$ and $\beta_{0}$ are two more parameters of the model. However, their influence on the dynamics is small as long as they are 


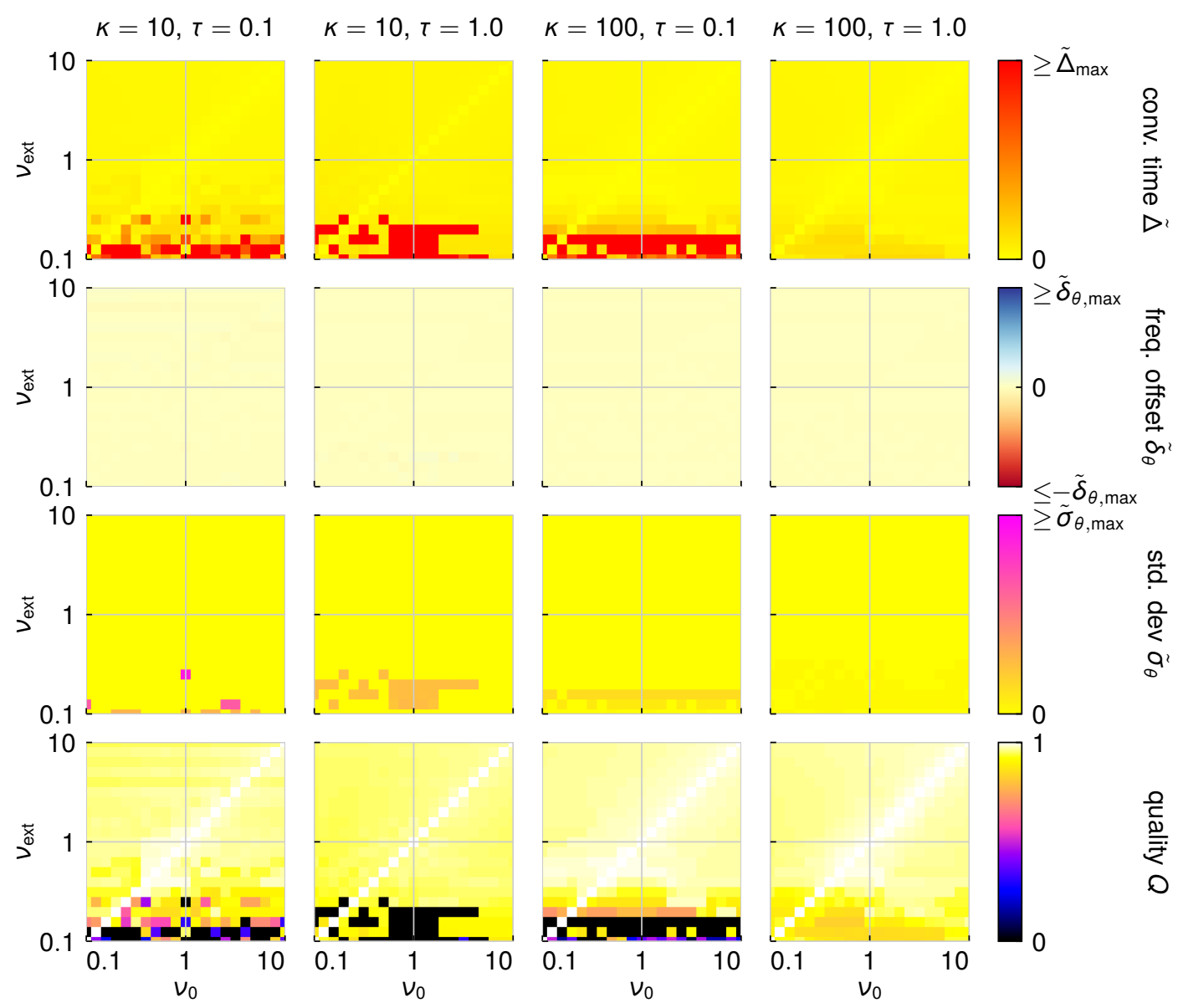

Figure 5.7: Adaptation quality measures for different parameter values of the Hopf oscillator with AFDC mechanism in the $\nu_{0}-\nu_{\text {ext }}$-frequency space. For every given parameter pair of the correlation learning rate $K$ and the time scale $\tau$, the respective columns show (from top to bottom), the relative convergence time $\tilde{\Delta}$, the relative frequency parameter offset $\tilde{\delta}_{\theta}$, the final standard deviation $\tilde{\sigma}_{\theta}$ of $\theta(t)$, and the combined quality index $Q$ in the frequency space spanned by the initial intrinsic frequency $\nu_{0}$ of the oscillator and the frequency $\nu_{\text {ext }}$ of the external stimulation. In all cases, the frequency variable learning rate $\eta$ has the value $\eta=1$. In comparison to the Hopf oscillator with AFO mechanism (Figure 5.4 ), here, a fixed set of parameter values allows high-quality adaptation processes within a wide range of frequencies. For $K=100$ and $\tau=1.0$, the quality index $Q$ attains values close to 1 for all sampled frequency configurations. Figure adapted from Nachstedt et al., (2017).

chosen sufficiently small. The analysis of the optimal parametrization of an AFDC oscillator therefore focuses on the $\tau-\kappa-\eta$-parameter space. We first discuss in detail the results for the Hopf oscillator with AFDC mechanism. Afterwards, we make sure that the findings also hold for the Van der Pol oscillator with AFDC mechanism.

We start by investigating the relative convergence time $\tilde{\Delta}$, the relative frequency offset $\tilde{\delta}_{\theta}$, the relative standard deviation $\tilde{\sigma}_{\theta}$, and the combined quality index $Q$ of the Hopf oscillator with AFDC mechanism in the space spanned by the initial intrinsic frequency $v_{0}$ and the frequency $v_{\mathrm{ext}}$ of the external stimulation. In contrast to the Hopf oscillator with AFO mechanism, fixed sets of parameter values of the Hopf oscillator with AFDC mechanism yield adaptation processes with high $Q$-values for a large range of frequencies $v_{0}$ and $v_{\text {ext }}$ (Figure 5.7). In particular the relative frequency offset $\tilde{\delta}_{\theta}$ is low for all tested parameterizations and frequencies. While the convergence time $\tilde{\Delta}$ is also low for most parameter values and frequencies, adaptation processes with low stimulation frequencies $v_{\text {ext }}$ turn out to require larger values of the correlation 
a Hopf oscillator with AFDC mechanism

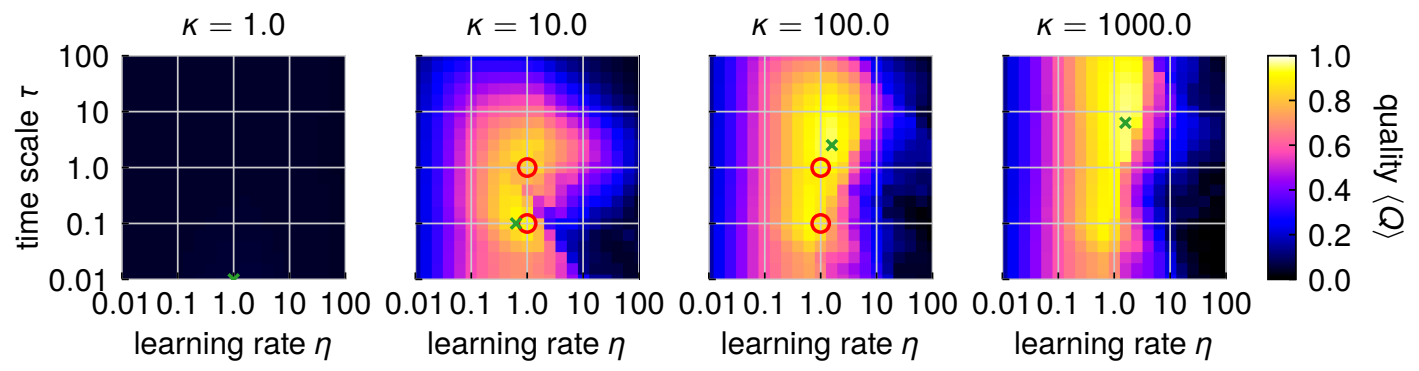

b

Van der Pol oscillator with AFDC mechanism

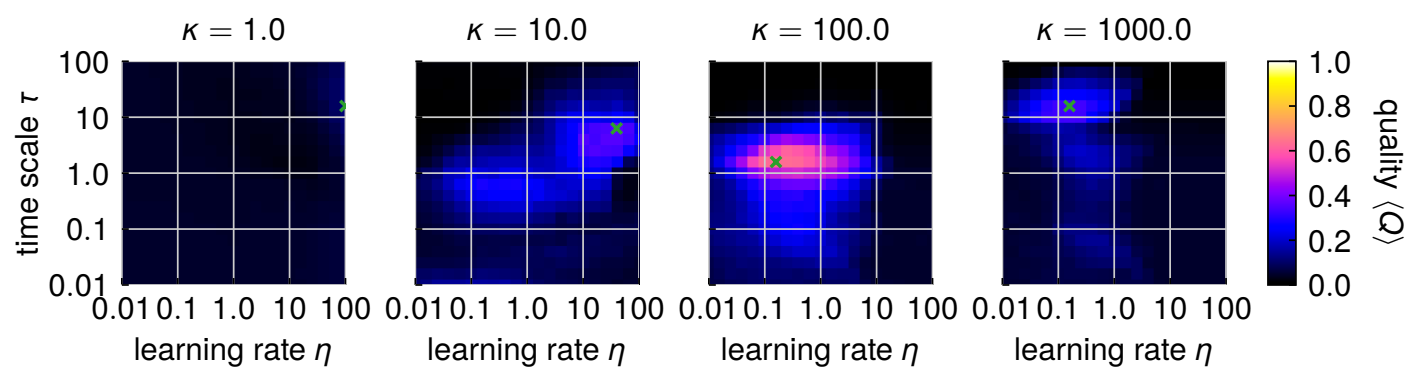

Figure 5.8: Frequency space averaged quality index $\langle Q\rangle$ for different parameter values of oscillators with the AFDC mechanism. The average adaptation quality index $\langle Q\rangle$ aggregates the quality index $Q$ of all combinations of the initial intrinsic frequency $\nu_{0}$ of the oscillator and of the frequency $\nu_{\text {ext }}$ of the external stimulation. The average is calculated over the logarithmically sampled frequency space defined by $0.1 \leq\left\{\nu_{0}, \nu_{\text {ext }}\right\} \leq 10$. (a) For sufficiently high values of the correlation learning rate $K$, we always find $(\eta, \tau)$-parameter values which yield a value of $\langle Q\rangle$ close to the maximum of one for the Hopf oscillator with AFDC mechanism. This corresponds to fast and precise adaptation processes within the complete space of sampled frequencies. The red circles indicate the four configuration of the Hopf oscillator shown in Figure 5.7. (b) For the Van der Pol oscillator with AFDC mechanism, the parameter range enabling a large average quality $\langle Q\rangle$ is more restricted. $(\mathbf{a}, \mathbf{b})$ The green crosses indicate the maximum values for the respective values of $K$. Figure adapted from Nachstedt et al., (2017).

learning rate $\kappa$ and the time scale $\tau$. These larger parameter values do not increase the value $\tilde{\Delta}$ for other areas of the investigated frequency space. Similarly, larger values of $\kappa$ and $\tau$ also result in smaller values of the relative standard deviation $\tilde{\sigma}_{\theta}$ for low values of $v_{\text {ext }}$. For instance, the parameterization $\eta=1.0, \kappa=100$, and $\tau=1.0$ results in quality values $Q$ close to one within the complete investigated $v_{0}-v_{\text {ext }}$-frequency space (fourth column in Figure 5.7).

The range of initial intrinsic frequencies $v_{0}$ and external stimulation frequencies $v_{\text {ext }}$ for which a given parameterization of the Hopf oscillator with AFDC mechanism achieves high values of the quality index $Q$ is significantly larger as found for the Hopf oscillator with AFO mechanism (compare Figure 5.4 and Figure 5.7). This results in higher values of the frequency space averaged quality value $\langle Q\rangle$. Investigating $\langle Q\rangle$ in the $\eta$ - $\tau$-parameter space for different values of $\kappa$ shows that, for sufficiently high values of $\kappa$, parameter values of $\epsilon$ and $\eta$ can be found which enable an average quality index $\langle Q\rangle$ close to the theoretical maximum of one (Figure $5.8 \mathrm{a}$ ). The best found value is $\langle Q\rangle \approx 0.96$. This is significantly larger than the best found value $\langle Q\rangle \approx 0.12$ for the Hopf oscillator with the AFO mechanism. The large value of $\langle Q\rangle$ corresponds to very fast adaptation processes with high precision for any combination of initial intrinsic frequencies $v_{0}$ and external stimulation frequencies $v_{\text {ext }}$ contained in the $v_{0}-v_{\text {ext }}$-frequency space spanning two orders of magnitudes.

Repeating the analysis for the Van der Pol oscillator with AFDC mechanism 
a

AFO mechanism
AFDC mechanism

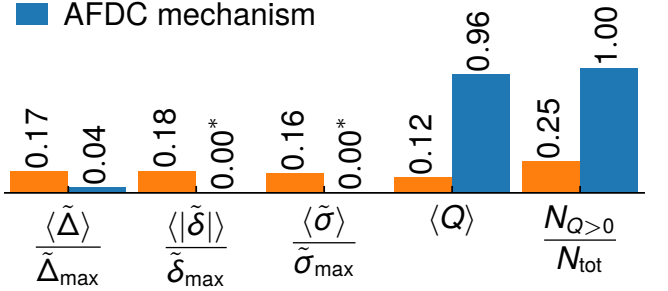

b

Van der Pol oscillator

AFO mechanism

AFDC mechanism

Figure 5.9: Average quality measures for the best found parameterizations of the oscillators with AFO mechanism and with AFDC mechanism. For both (a) the Hopf oscillator and (b) the Van der Pol oscillator, the AFDC mechanism outperforms the AFO mechanism in terms of all defined quality measures. Note that the average relative convergence time $\langle\tilde{\Delta}\rangle$, the average absolute relative frequency variable offset $\left\langle\left|\tilde{\delta}_{\theta}\right|\right\rangle$ and the average frequency variable standard deviation $\langle\tilde{\sigma}\rangle$ include only values from $\left(\nu_{0}, \nu_{\text {ext }}\right)$-frequency pairs for which the combined quality index $Q$ has a nonzero value. The ratio of the number $N_{Q>0}$ of these frequency pairs and the number $N_{\text {tot }}$ of all testes frequency pairs is shown on the very right. All numbers are rounded. (a) The best found parameter values for the Hopf oscillator with AFO mechanism are $\epsilon=\eta \approx 15.8$. The best found configuration of the Hopf oscillator with AFDC mechanism is $\tau \approx 3.98, \eta \approx 1.58$, and $K \approx 398\left(\beta_{0}=0.0\right.$ and $\epsilon_{0}=0.01$ ). (b) For the Van der Pol oscillator with AFO mechanism, the best parametrization is $\epsilon \approx 0.0158$ and $\eta=1.0$. The Van der Pol oscillator with AFDC mechanism performs best for $\tau \approx 1.58, \eta \approx 0.158$, and $K \approx 100\left(\beta_{0}=0.0\right.$ and $\left.\epsilon_{0}=0.01\right)$. ( $\left.{ }^{*}\right)$ Values shown as 0.00 are too small to be resolved in the figure and are stated in the following. Hopf oscillator with AFDC mechanism: $\left\langle\left|\tilde{\delta}_{\theta}\right|\right\rangle / \tilde{\delta}_{\theta, \max } \approx 5.3 \times 10^{-7}$ and $\left\langle\tilde{\sigma}_{\theta}\right\rangle / \tilde{\sigma}_{\theta, \max } \approx 8.5 \times 10^{-8}$. Van der Pol oscillator with AFDC mechanism: $\left\langle\tilde{\sigma}_{\theta}\right\rangle / \tilde{\sigma}_{\theta, \max }=2.5 \times 10^{-3}$. Figure adapted from Nachstedt et al., (2017).

shows that, compared to the Hopf oscillator with AFDC mechanism, the parameter range enabling fast and precise adaptation is more restricted (Figure $5.8 \mathrm{~b}$ ). Still, we find a configuration which results in a frequency space averaged quality index of $\langle Q\rangle \approx 0.63$. Compared to best value found for the Van der Pol oscillator with AFO mechanism $(\langle Q\rangle \approx 0.08)$, this is still a significant increase.

\subsubsection{Quantitative Comparison of the AFO and the AFDC mechanism}

In the previous section, we have demonstrated the ability of the AFDC mechanism to enable fast and precise adaptations within a wider frequency regime than the AFO mechanism. In particular, we compared the best found frequency space averaged quality index $\langle Q\rangle$. Here, we compare the individual quality measures of the corresponding optimal parameter configurations. This quantitative comparison further underlines the qualitative difference between the two adaptation mechanisms.

For the Hopf oscillator with AFO mechanism, the parameter value pair $\epsilon=\eta \approx$ 15.8 performs best and results in an average quality index value of $\langle Q\rangle \approx 0.12$ (Figure 5.9 a). Still, only the adaptation processes for roughly $25 \%$ of the investigated $\left(v_{0}, v_{\text {ext }}\right)$-frequency pairs are characterized by a nonzero quality index in this case. When restricted to these cases, the average relative convergence time $\tilde{\Delta}$, the relative frequency variable offset $\tilde{\delta}_{\theta}$, and the standard deviation $\tilde{\sigma}$ normalized by the respective maximum values for the quality index calculation take values in between 0.16 and 0.18. The best tested configuration for the Hopf oscillator with AFDC mechanism is $\tau \approx 3.98, \eta \approx 1.58$, and $\kappa \approx 398$. For this parametrization, all $\left(v_{0}, v_{\text {ext }}\right)$-frequency pairs lead to adaptation processes with nonzero quality indices $Q$ and their frequency 
space averaged quality index $\langle Q\rangle \approx 0.96$ is close to maximum. The individual quality measures are all significantly lower (hence better) than in the case of the AFO mechanism.

Similar observations do also apply for the comparison between the best configuration of the Van der Pol oscillator with AFO mechanism and the Van der Pol oscillator with AFDC mechanism (Figure 5.9b). Due the highly nonlinear limit cycle of the Van der Pol oscillator, however, also the best parametrization of the AFDC version of this oscillator only results in nonzero quality index values for approximately $79 \%$ of the investigated frequency pairs. Still, this is a considerably larger ratio than the $15 \%$ found for the AFO mechanism. Similar to the Hopf oscillator, also the average individual quality measures $\langle\tilde{\Delta}\rangle,\left\langle\left|\tilde{\delta}_{\theta}\right|\right\rangle$ and $\left\langle\sigma_{\theta}\right\rangle$ calculated for the $N_{Q>0}$ nonzero quality index cases are all lower (better) for the AFDC version of the Van der Pol oscillator than for the AFO mechanism. Note that the maximum values $\tilde{\Delta}_{\max }, \tilde{\delta}_{\theta \text {, max }}$ and $\tilde{\sigma}_{\theta, \max }$, which we use to calculate the quality index $Q$, are larger for the Van der Pol oscillator than for the Hopf oscillator.

\subsection{Discussion}

In this chapter, we propose a mechanism that may explain how short-lasting transient signals of the WM system may be used to evoke adaptive long-lasting time-dependent neuronal signal as required, for instance, in the control of locomotion. We model CPGs as nonlinear oscillators and develop a mechanism that, in contrast to the previously existing AFO mechanism (Righetti et al., 2006), allows to adapt the frequency of the periodic attractor in these oscillators fast and precisely within a wide frequency range in a self-organized way. This verifies the third sub-hypothesis of this thesis and shows that WM may evoke long-lasting signals of varying frequencies by only transiently producing few periods of this respective signal.

The AFO mechanism, as introduced by Righetti et al., (2006), is a powerful method to adapt the frequencies of different types of oscillatory systems to the frequency of an external signal. We show, however, that the trade-off between speed and precision inherent to the AFO mechanism does not allow to find parameter values which enable fast and precise adaptations within a wide range of frequencies. Therefore, we develop a new adaptation mechanism which relies on the dynamic adaptation of the coupling strengths between the oscillatory system and an external periodic stimulation. The dynamics of the adaptive coupling strengths temporally increases the influence of the external stimulation on the system and thereby increase the adaptation rate. Once the intrinsic frequency of the oscillator is close to the one of the external stimulation, the influence of the latter on the oscillator is reduced. This enables a precise adaptation toward the end of the adaptation process. As a consequence, this adaptation through fast dynamical coupling (AFDC) allows fast and precise adaptation within a wide range of frequencies with a single fixed set of parameter values.

Mechanisms to dynamically adjust the coupling strengths of oscillators have been studied before in the context of networks of regular, i.e., non-adaptive, oscillators with different intrinsic frequencies (Ren and Zhao, 2007). Also in this study, the adaptive coupling strengths are strong in the beginning, when the oscillators are still desynchronized, and are weak once synchronization is achieved. As in this study the oscillators do not adapt their intrinsic frequencies, however, the coupling strengths cannot ultimately decay to zero like they do in the AFDC mechanism. 
Within this chapter, the AFO mechanism and the AFDC mechanism are studied by applying them to the harmonic Hopf oscillator and the highly non-harmonic Van der Pol oscillator. In both cases, the AFDC mechanism clearly outperforms the AFO mechanism in the employed benchmark test. Still, the adaptive versions of the Van der Pol oscillator in general performs weaker than the equivalent versions of the Hopf oscillator. In order to verify the generality of the AFDC mechanism, it remains to be investigated whether the mechanism can also be applied to other standard types of nonlinear oscillators like the Rayleigh-oscillator (Rayleigh, 1877), the Duffing oscillator (Duffing, 1918), or the FitzHugh-Nagumo oscillator (Fitzhugh, 1961).

Considering possible biological implementations of the AFDC mechanism, the interaction of the fast transient dynamics of the adaptive coupling strengths and the slower but permanent adaptation of the intrinsic frequency of the oscillator resembles the interplay of short-term plasticity (Zucker and Regehr, 2002) and long-term plasticity (Wood et al., 2011) in neuronal systems. In Nachstedt et al., (2017), we propose an implementation of the AFDC rule that employs synaptic plasticity to modify the output frequency of an abstract neuronal CPG consisting of only two fully connected point-like and rate based neurons (Pasemann et al., 2003). In this neuronal implementation of the AFDC mechanism, the adaptive coupling strengths translate into synaptic connections whose transmission efficacy is subject to transient correlation-based plasticity. The adaptation of the intrinsic frequency, in turn, constitutes a long-term modification of the network dynamics (Nachstedt et al., 2012). Therefore, when applied to a neuronal system, the AFDC mechanism describes the emergence of fast and precise adaptation behavior based on the interplay of shortterm and long-term plasticity processes (Nachstedt et al., 2012). This agrees with other results indicating the relevance of this interplay of short-term and long-term plasticity for fast network reconfiguration in biological motor control (Nadim and Manor, 2000).

Nevertheless, CPGs in biological neuronal system that adapt their oscillation frequencies on short timescales have not yet been reported. Interestingly, however, it has been shown that the concentration of the neuromodulator serotonin is able to modulate the output frequency of a CPG over a wide frequency range (Harris-Warrick and Cohen, 1985). However, due to the ongoing diffusion processes, it is not clear whether a neuromodulator concentration can account for the permanent frequency adaptation implemented by the AFDC mechanism.

As discussed earlier, CPGs play an important role in the control of the different types of locomotion including legged locomotion. It is known that the leg frequency during locomotion is tightly coupled to the resonant frequency of the freely swinging leg (Holt et al., 1990). This enables animals to achieve optimal energy efficiency (Ahlborn and Blake, 2002). Actually, it has also been proposed that animals might actively modify the resonant frequency of their legs to allow for different locomotion speeds (Ahlborn and Blake, 2002). The adaptation of the intrinsic frequency of a CPG toward the resonant frequency of the controlled system has been repeatedly modeled (Verdaasdonk et al., 2006; Verdaasdonk et al., 2009). The AFDC mechanism also offers an explanation how animals might achieve fast and precise adaptation of their CPGs in this context. This opens up the possibility to rely on few distinct CPGs being responsible for the complete range of available locomotion speeds.

Following the path of transferring successful biological concepts to the field of robotics (Pfeifer et al., 2007), the time-discrete predecessor of the general AFDC mechanism has been shown to be functional in different robotic control tasks including self-organized control of a snake-like robot (Nachstedt et al., 2013), adaptive control of a robot leg with compliant tarsus (Canio et al., 2016b), and bipedal locomotion 
which is robust against global loss of sensory feedback (Canio et al., 2016a). As the here developed general AFDC mechanism is applicable to all kinds of nonlinear oscillators, it may allow further robotic applications relying on different kinds of CPGs (Ijspeert, 2008; Nassour et al., 2014; Santos et al., 2017).

In the context of this thesis, the AFDC mechanism provides a mechanism which allow a CPG to adapt its output signal based on transient signals arriving from the neuronal system implementing WM. Still, the AFDC mechanism does only allow to appropriately adjust the frequency of the CPG output signal. A complete interaction schema between WM and CPGs does also have to explain how to dynamically adapt the shape of the produced signal. Interestingly, it has been shown that a set of parallel operating oscillators with AFO mechanism is able to adaptively synthesize complex periodic signals (Righetti et al., 2009). If this schema can be transferred to oscillators with the AFDC mechanism, it might provide an explanation how a CPG can adapt both the frequency and the shape of its output signal based on short transient signals from the WM system. 


\section{Chapter 6}

\section{Discussion and Future Work}

The ability to form memories of past stimuli is fundamental to the generation of complex behavior (Eichenbaum, 2011). At the same time, the neuronal mechanisms implementing the processing and storage of past information across different time scales are still not known. In particular, it is not clear whether the neuronal circuits that implement working memory (WM) are dominated by dynamics that relies on different attractor states or by purely transient dynamics. Experimental (e.g. Fuster and Alexander, 1971; Funahashi et al., 1989; Goldman-Rakic, 1995; Rainer and Miller, 2002; Constantinidis and Wang, 2004; Sreenivasan et al., 2014) as well as theoretical studies (e.g. Durstewitz et al., 2000; Compte et al., 2000; Rabinovich et al., 2008a; Stokes et al., 2013; Drover, 2014; Barak and Tsodyks, 2014) are not conclusive and yield support for both seemingly contradictory assumptions.

In this thesis, we evaluate the hypothesis that complex and robust WM operation arises from the interaction of transient neuronal dynamics with self-organizing attractor states in other neuronal memory systems. In order to evaluate this hypothesis, we derive three necessary sub-hypotheses which we verify separately. First, we demonstrate that robust WM indeed requires both transient dynamics and distinct attractor states (Chapter 3). Second, we show that these distinct attractor states may be formed in a self-organized way in the long-term memory (LTM) based on elementary synaptic plasticity processes (Chapter 4). Finally, we develop a mechanism that is able to transform short-lasting transient outputs from WM into long-lasting time-dependent signals in a self-organized way (Chapter 5).

In the following, we discuss the results which we obtain in this thesis with respect to the formulated hypotheses. More detailed discussions of the individual results are given at the end of the respective chapters.

\section{Interplay of Transient Dynamics and Attractor States in WM}

As stated above, experimental findings on the type of neuronal dynamics that underly WM are diverse. Our first sub-hypothesis states that only the combination of both, complex transient dynamics and distinct attractor states, allows for robust WM operation. In order to validate this hypothesis, we first show that purely transient neuronal dynamics does not enable robust WM operation. We demonstrate that a transient network model is able to solve a typical WM task only for predictable stimulus timing (Section 3.2.1 and 3.2.2). This is in contrast to the results reported for human subjects whose performance in this task does not depend on the predictability of the stimulus timing (Koppe et al., 2014). Importantly, previous studies using networks with purely transient dynamics to solve WM tasks did not systematically evaluate the influence of unpredictable stimulus timing on the performance (e.g. Barak et al., 2013; Dasgupta et al., 2013; Barak and Tsodyks, 2014; Cheng et al., 2015). The break-down of the performance in the case of unpredictable stimulus timing is 
related to the overlap of the trajectory bundles of the system which represent the different stimulus histories (Section 3.2.4). This phenomenon is very generic and we expect that it can be generalized from the class of network models used here to other network models which store information in a purely transient manner (for a review, see Buonomano and Maass, 2009). Thus, we conclude that robust WM cannot be implemented by purely transient neuronal dynamics.

To enable the network to solve the WM task with unpredictable stimulus timing, we introduce additional feedback loops which represent the relevant information about the current stimulus history (Section 3.2.3). Such feedback loops have been proposed before to enable the longer storage of information in reservoir networks (Maass et al., 2007; Pascanu and Jaeger, 2011). They have not been considered, however, in the context of unpredictable stimulus timing as done here. The feedback loops effectively structure the phase space of the system by introducing distinct attractor states (Section 3.2.4). As a result, the trajectory bundles representing different stimulus histories remain well separated also in the case of unpredictable stimulus timing. Accordingly, the network model is now able to perform the WM task robustly irrespective of the predictability of the stimulus timing. Importantly, this network model employs both transient dynamics and attractor states to solve the WM task.

The supporting role of persistent activity for the maintenance of information in WM has recently been experimentally confirmed (Kamiński et al., 2017). Importantly, in this experiment, it was found that information-specific persistent activity just constitutes one fraction of the total neuronal activity. Similar to the requirements identified here, stable or attractor dominated encoding of information coexists with dynamic and transient encoding (Murray et al., 2017). The dynamic components of the coding are especially strong during the cue and the early delay period as also observed for the transient dynamics in our model (Murray et al., 2017). Interestingly, maintenance of information in WM without persistent activity is mostly found for experimental paradigms which use fixed maintenance durations (e.g. Watanabe and Funahashi, 2007; Barak et al., 2010). Thus, it seems that the brain relies on transient information storage when the temporal structure of the task is predictable and switches to more robust storage mechanism otherwise (Stokes, 2015). This agrees well with our results. When the temporal structure of the task is predictable, the network can rely on attractor-less transient storage of the information to perform this task. If the temporal structure is unpredictable, on the other hand, attractor states in other memory systems have to be employed. This explains why during performing a WM task with unpredictable stimulus timing, a higher activity in multiple brain regions was recorded than solving the same WM task with predictable stimulus timing (Koppe et al., 2014). Switching in between these two modes may be beneficial in terms of energetic efficient processing (Laughlin, 2001).

The attractor states which we introduce to allow robust memory storage in the presence of unpredictable stimulus timing are formed via additional feedback loops. We can interpret the signals that the recurrent network receives via these feedback loops as self-generated context signals (Santiago, 2004; Sussillo and Barak, 2013). It has been shown that a transient network model that receives such context signals reproduces population dynamics similar to dynamics observed in the PFC (Mante et al., 2013). Thus, transient dynamics modulated by a constant context or feedback signal might indeed be a plausible mechanism to explain complex dynamical computations in the brain.

The beneficial effect of the attractor states emerges from the additional structure introduced into the phase space of the neuronal dynamics. Accordingly, we propose that also other mechanism that modify the neuronal dynamics in a similar way may 
allow robust WM performance in tasks with unpredictable temporal structures. In particular, this includes attractor relics as employed in saddle point and heteroclinic networks (Rabinovich et al., 2008b; Gros, 2009; Bick and Rabinovich, 2009) as well as short-term synaptic plasticity effects (Mongillo et al., 2008; Rose et al., 2016a). As long as these processes reflect past stimuli reliably on time scales that are significantly longer than the time scale of the neuronal dynamics, they effectively might behave like attractor states for the evolution of the neuronal dynamics.

At this point, we have shown that a purely transient network is not able to solve the n-back task with unpredictable temporal structure and that a network that exploits both, transient dynamics and distinct attractor states, can solve this task. To completely verify our first sub-hypothesis, we finally show that the n-back task cannot be solved by a network that is solely relying on different attractor states. We do so by investigating the performance of the reservoir network with two feedback loops in a simplified version of the WM task with predictable stimulus timing (Section 3.2.5). Although all the relevant information is available to the network, its ability to produce the time-dependent readout signal is reduced once the network dynamics is close to the attractor state at the end of a transient trajectory. Introducing an unspecific recall stimulus leads to a new transient trajectory and restores the computational power of the network. Hence, time-dependent computation cannot be implemented without complex transient dynamics in the network. As WM requires the ability to perform complex computations (Baddeley, 2012; Revlin, 2013), the underlying neuronal system must be using complex transient dynamics.

In summary, these findings verify our first sub-hypothesis in this thesis: Only the combination of both distinct attractor states and transient neuronal dynamics enables WM to robustly store information and to perform complex computations. It remains an open question, however, whether these distinct attractor states might also be evoked by non-stationary processes evolving on slower time scales.

\section{Self-Organized LTM Representations as Attractor States for WM Operation}

The verification of the first sub-hypothesis yields to a subsequent research questions: Given that WM requires meaningful attractor states for robust performance on unreliably timed input stimuli, there needs to be a mechanism by which these attractor states are formed in a self-organized way in a neuronal network. Following the main hypothesis of this thesis, we propose that these attractor states emerge from the interaction of WM with other memory systems, in particular with LTM. This requires us to verify the second sub-hypothesis stating that relevant attractor states may be formed and assigned reliably in a self-organized way in the LTM system.

To verify the second sub-hypothesis, we propose a network model that is based on a recurrent area which receives feedforward signals from a dedicated input area (Section 4.1.1). We show that in this network, representations of different input stimuli are formed and assigned to these stimuli in a self-organized way (Section 4.2.1). The time scale of these processes is defined by the time scales of Hebbian plasticity and synaptic scaling which evoke synaptic changes that last for hours and days (Mayford et al., 2012; Tetzlaff et al., 2012a). Therefore, our model indeed operates on the time scales relevant for LTM. The stimulus representations which are formed in the network are functional Hebbian cell assemblies (CAs) (Hebb, 1949; Palm et al., 2014, , Section 4.2.3). The self-organization is based on the interplay of Hebbian plasticity (Hebb, 1949) and synaptic scaling (Turrigiano et al., 1998) at both the recurrent synapses and the feedforward synapses. By deriving a reduced population model, we investigate the equilibrium structure which underlies the 
network dynamics (Section 4.3). To ensure that the formation of CAs in our model does not depend on specific features of the input stimuli, we show that it is robust with respect to the number of active neurons in the input area (Section 4.2.2) and with respect to the firing rate of these neurons (Section 4.3.2). Thus, this confirms our second sub-hypothesis: The interaction of Hebbian plasticity and synaptic scaling enables the reliable self-organized formation and assignment of attractor states in LTM.

According to the main hypothesis of this thesis, the self-organized attractor states in the LTM system provide the attractor states required by WM to robustly process unreliable input stimuli. This notion is supported by recent experimental evidence showing that the storage of WM information is distributed across multiple brain regions which are not specifically dedicated to WM (Christophel et al., 2017), i.e., in brain regions other than the prefrontal cortex (PFC). Brain regions which are involved in processing a specific kind of information in non-WM tasks are also active during WM maintenance of the same kind of information (D'Esposito and Postle, 2015). Phenomenologically, the existence of LTM representations has been shown to support the operation of WM (Hulme et al., 1991; Poirier et al., 2011). All these findings suggest that the PFC as central WM unit uses existing LTM representations of stimuli to maintain the respective information. In terms of the WM model of Baddeley, (2003) (Section 2.4), the PFC might thus rather correspond to the central executive and not to any of the three short term buffer systems (D'Esposito and Postle, 2015).

Note that in Section 3.2.3, we introduce attractor states which represent the identities of the two most recent stimuli in the form of ongoing activity. The CAs formed in our LTM model in Chapter 4, however, are input-dependent attractors of the system. Without additional excitation, they do not show persistent activity. A possible mechanism which allows the CAs to represent past stimuli in a way similar to the additional readout units introduced in Section 3.2.3 might be based on an ongoing low-level excitatory input during the delay phase of a WM task. This low-level excitatory input would constitute a kind of gating mechanism as already proposed by (Cowan, 1988). Alternatively, adapting the level of inhibition received by neurons in the recurrent layer of our network model could allow CAs to show persistent activity. Dynamical switching between input-driven attractors and persistent activity states might be mediated, for instance, by mechanism of feedforward inhibition (Buzsáki, 1984; Large et al., 2016).

Apart from being input-dependent attractors, the CAs formed in our LTM model also only correspond to exactly one stimulus. Thus, in order to keep multiple items robustly in WM, there need to be attractor states in which multiple CAs are active simultaneously. Due to the strong competition introduced by the global inhibition, our network model currently does not allow a reliable way to achieve this (Section 4.3.2). In other LTM models operating with similar attractor dynamics, it has been shown that the number of simultaneously active representations can be controlled by the level of the global inhibitory signals (Romani et al., 2013). It remains to be investigated whether such a mechanism would also work in the LTM model discussed within this thesis. Alternatively, introducing local inhibition mechanisms (Fukai and Tanaka, 1997; Harris and Mrsic-Flogel, 2013; Stevens et al., 2013; Tetzlaff et al., 2013) which limit the growth of a given CA without preventing other non-overlapping CAs to get active might also allow the concurrent activity of multiple CAs. Note that ultimately, this concurrent activity of multiple CAs would also have to encode the temporal order of the respective stimuli. For instance, in the n-back task, it is required to be able to distinguish the last stimulus from the second-last stimulus. Different models of the storage of temporal and serial order in the brain have been 
proposed (Henson, 1998; Burgess and Hitch, 1999; Brown et al., 2000; Hurlstone et al., 2014). It remains to be investigated if any of these mechanisms can be transfered to the here-described model.

Alternatively to storing information in terms of ongoing activity, it has been proposed that WM may also rely on specific patterns of synaptic weights that arise from short-term synaptic plasticity (Mongillo et al., 2008; Barak and Tsodyks, 2014; Stokes, 2015; Wolff et al., 2017). These specific patterns are called activity-silent representations. The information stored in these activity-silent representations is recalled when the modified synaptic weights influence the emerging neuronal dynamics. This may be guided by attention mechanisms (Rose et al., 2016b). In comparison to persistent activity, activity-silent representations are assumed to be less energetically expensive (Stokes, 2015). In this framework, the number of items that can be kept in WM can be directly related to parameters of the short-term plasticity process and regulated by external excitation (Mi et al., 2017). It is proposed that activity-silent representations underly unconscious WM, i.e., the short-term maintenance of information without the subject being aware of this information (Trübutschek et al., 2017). Accordingly, both persistent activity representations as well as activity-silent representations contribute to WM operation (Silvanto, 2017). The hypotheses in this thesis are compatible with activity-silent representations. Introducing short-term synaptic plasticity into the LTM model in Chapter 4 might increase the level of excitability of a given CA after its activation. A certain level of activity in the WM system might then suffice to activate this CA. Due to the pattern completion properties of the CA (Section 4.2.3), the resulting signals from the LTM system to the WM system might influence the transient dynamics in the WM system in a similar prototypical way as the signals mediated by the activity feedback loops (Section 3.2.3). Thus, the mechanism of activity-silent representations conforms to our main hypothesis stating that self-organized attractor states in the LTM system support the transient dynamics in the WM system.

In summary, the interaction of Hebbian plasticity and synaptic scaling enables the self-organized formation and allocation of attractor states which represent different stimuli in LTM. These attractor states might support the storage of information in LTM either by persistent activity or in the form of activity-silent representations with a higher level of excitability. Regarding the possibility of persistent activity, it remains to be investigated how multiple CAs may be kept active simultaneously in the network and how this concurrent activity may represent the temporal order of the corresponding stimuli.

\section{Unpredictable Timing in the Interaction of WM and LTM}

In Chapter 3, we consider unpredictable stimulus timing in an established WM benchmark task, namely the n-back task (Jaeggi et al., 2003; Conway et al., 2005; Owen et al., 2005; Kane et al., 2007b; Jaeggi et al., 2010). As such, the n-back task does not require much contributions of other memory systems like LTM, at least in the case of a predictable temporal structure. In everyday life, however, WM and LTM are known to continuously interact and support each other's operation (Baddeley et al., 1988; Hulme et al., 1991; Poirier et al., 2011; Marton and Eichorn, 2014). The neuronal mechanisms underlying this interaction have not yet been analyzed intensively (but see Burgess and Hitch, 2005).

The fact that WM and LTM interact continuously shows that WM needs to be able to operate on unpredictably timed input stimuli already during the internal signal exchange in between different neuronal systems in the brain. Like the model of selforganized CAs in Section 4, LTM is generally assumed to operate based on attractor 
dynamics (Hopfield, 1982; Gerstner and Kistler, 2002; Wood et al., 2011; Tetzlaff et al., 2013). Thus, recalling a specific item from LTM corresponds to the convergence of the system to the respective attractor state. The duration of this convergence determines the retrieval time of the respective memory item. The retrieval time may vary from recall to recall depending on the state of the memory system and the recall stimulus. This yields a distribution of retrieval times which has been studied theoretically (Kohring, 1990) as well as phenomenologically (Juola et al., 1971). Given the continuous interaction of WM and LTM, the WM system has to be able to robustly operate on signals retrieved from LTM despite of the varying retrieval times.

Thus, the unpredictability of retrieval times in LTM provide further evidence for the importance of robustness with respect to unpredictable stimulus timing in the neuronal system implementing WM.

\section{Transforming Transient Activity to Long-Lasting Signals with adaptive CPGs}

Having shown that computation and the production of complex output signals in WM depends on transient dynamics, a second follow-up research question arises. Given that the transient dynamics represents the transition of the WM system from one attractor state to the next, its duration is coupled to the speed of operation of WM. Shorter transients enable a faster succession of attractor states and therefore a higher rate of different computations. At the same time, many actions that may be triggered by WM require much longer-lasting control signals. For instance, walking or running requires ongoing neuronal control. Thus, the question arises how shortlasting transient signals can be used to control longer-lasting output signals.

According to our third hypothesis, one possible solution to this problem is given by central pattern generators (CPGs) (Hooper, 2001; Ijspeert, 2008) which are able to adapt fast and precisely the properties of their output signal based on short input signals. Short transient signals produced by WM might trigger a CPG to adapt its attractive limit cycle such that its output signal mimics the prototypical signal received from WM. In order to enable CPGs to accomplish this task, we introduce a new kind of adaptation mechanism which is based on adaptation through fast dynamical coupling strengths (AFDC). In contrast to the existing adaptive frequency oscillator (AFO) mechanism (Righetti et al., 2006, Section 5.2), the AFDC mechanism is able to adapt the frequency of an oscillatory system fast and precise within only few periods of input activity (Section 5.3). When the input stimulation vanishes, the oscillatory system keeps oscillating at the adapted frequency. Thus, this mechanism is able to transform the frequency of a short-lasting, i.e., transient signal into a long-lasting time-dependent signal of the same frequency. This verifies our third sub-hypothesis.

For the AFO mechanism, it has been shown that multiple parallel adaptive oscillators are able to synthesize complex periodic signals (Righetti et al., 2009). Basically, this is based on a Fourier decomposition of the incoming signal where every oscillator automatically adapts its frequency to one of the significant Fourier components. This shows that, in general, frequency adaptation of single oscillators suffices to reproduce periodic signals of basically arbitrary shape (Righetti et al., 2009). We expect that a similar structure composed of oscillators with AFDC mechanism would also significantly speed up the adaptation towards arbitrary periodic signals.

In this thesis, we consider CPGs on the very abstract level of non-linear limit cycle systems, i.e., systems with periodic attractors. A variety of more detailed biophysical models and studies have been proposed that show how CPGs may be implemented in neuronal networks (Brown, 1911; Pearson and Duysens, 1976; Miller and Scott, 
1977; Matsuoka, 1985; Hellgren and Grillner, 1992; Tråvén et al., 1993; Hooper, 2001; Guertin, 2009; Guertin, 2013). In general, the AFDC mechanism is applicable to any CPG model as long as there is a parameter available which allows to monotonically control the frequency of the produced signal. However, the comparison between the adaptations of the Hopf oscillator with AFDC mechanism and the Van der Pol oscillator with AFDC mechanism also show that oscillatory systems with complex limit cycles are more difficult to adapt. Thus, it remains an open question in how far the AFDC mechanism is actually able to adapt the frequencies of complex biological CPG models.

In summary, the AFDC mechanism developed in this thesis provides a possible explanation for how short-lasting transient activity in WM may evoke long-lasting periodic control signals by self-organized adaptation of the periodic attractors of CPGs. It remains to be investigated in how far this mechanism can be transferred to more realistic CPG models.

\section{Evaluation of the Main Hypothesis}

As discussed above, in this thesis, we successfully verify all of our three initially formulated sub-hypotheses: First, we show that WM operation depends on both transient dynamics and attractor states for robust information storage and complex computation (sub-hypothesis 1). Second, we demonstrate that attractor states representing different stimuli can be formed and assigned in a self-organized way by Hebbian plasticity and synaptic scaling in the LTM system (sub-hypothesis 2). Finally, we also develop a mechanism that allows short-lasting transient signals to fast and precisely adapt the frequencies of periodic attractors of CPGs (sub-hypothesis 3). The verification of these three sub-hypotheses is an important prerequisite for the verification of the main hypothesis. To fully verify the main hypothesis, however, the functionality of the interaction of transient neuronal dynamics with stable attractor states in the LTM system and adaptive periodic attractor states in CPGs remains to be actually shown. The solutions developed during the verifications of the three sub-hypotheses of this thesis provide valuable insights about promising approaches to implement this interaction in a unifying neuronal network model.

\section{Outlook}

The presented models and the corresponding results and insights provide multiple possible starting points for future works. We already mentioned a couple of open questions regarding the individual memory systems which we investigated in this thesis: Regarding the neuronal dynamics underlying WM, we need to verify in how far also attractor relics (Rabinovich et al., 2008b; Gros, 2009; Bick and Rabinovich, 2009) or short-term synaptic plasticity processes (Mongillo et al., 2008; Rose et al., 2016a) may influence transient neuronal dynamics in a way that allows solving WM tasks with temporal unpredictable structure. In the LTM model, we have to explore possible mechanisms which allow to reliably control the simultaneous activity of multiple CAs in the recurrent network and to represent their temporal structure. Finally, concerning the AFDC mechanism for CPGs, we need to evaluate in how far the AFDC mechanism is applicable to complex biophysical models of CPGs and whether it can be generalized to adapt also the shape of the produced periodic output signal.

Apart from these research questions for the individual memory components, there are also various open questions regarding the interaction of these components. In 
this thesis, we analyze models of WM, LTM and CPGs and show that these three models provide the abilities required for beneficial interaction between them. The actual interaction of these model, however, remains to be demonstrated.

In a first step, the transient WM model and the self-organized LTM model might be combined. By repeated presentation of stimuli to this combined system, CAs representing these stimuli would emerge in the LTM system. We might then show that these representations can be used by the transient reservoir network model to solve an n-back task with unpredictable temporal structure (Koppe et al., 2014) operating on these stimuli.

In a second step, we might demonstrate the interaction between WM and CPGs. For this, we might train a reservoir network to produce transient periodic signals with a frequency that depends on an incoming input stimulus. This transient signal could then be transmitted to one or multiple CPGs with AFDC mechanism which adapt their periodic output signal accordingly. By training the reservoir network in parallel to also solve an n-back task, a so-called cognitive-motor dual-task could be modeled (Leone et al., 2017). In one particular setup of this task, it has been shown that human subjects are able to solve an n-back task well above chance level while at the same time performing periodic ankle movements (Johannsen et al., 2013). We predict that the interaction of a WM network model with transient dynamics and CPGs with AFDC mechanism should be able to reproduce this ability. After the initial control of the periodic ankle movement, the transient network model would be released from the ongoing active control of the movements by appropriately adjusting the output signals of the responsible CPGs. Arising deviations of the CPG frequency resulting, for instance, from sensory feedback, might still require occasional control by WM and account for a reduced performance in the n-back task (Johannsen et al., 2013). Based on this model, we might, for instance, derive experimental predictions regarding the consequences of an instructed change of the movement frequency.

In a final step, all three neuronal memory system might be combined to solve and analyze complex WM tasks, for instance, in closed-loop scenarios (Potter et al., 2014). As an example, we might design a scenario in which an agent has to integrate multiple sequentially and temporally unpredictably occurring stimuli and, based on the combination of the received stimuli, adapt its locomotion behavior. Here, LTM would allow to form representations of the relevant stimuli in a self-organized way. The representations would allow to robustly store the recently occurred stimuli in WM. In conjunction with the transient dynamics evoked by these stimuli, this would enable WM to produce a specific time-dependent transient signal controlling the first few periods of the new locomotion behavior. This signal, in turn, would result in the adaptation of CPGs with AFDC mechanism which would afterwards allow the ongoing control of this locomotion. Thus, this agent would be able to adapt its behavior based on a possibly complex function of the received stimuli.

Apart from these possible future modeling approaches, the hypothesis of this thesis also yields an interesting experimental prediction. Note that in the n-back study with unpredictable stimulus timing as performed by Koppe et al., (2014), the subjects were operating on well-known visual stimuli. Hence, we can assume that these stimuli were able to activate specific attractor states in the LTM system. According to our hypothesis, this explains the robustness of the performance even in the case of unpredictable stimulus timing. This robustness should vanish, however, if the n-back task is performed on stimuli which are not able to activate existing representations in LTM. For instance, these might be given by abstract images that cannot be decomposed into known geometrical shapes. We predict that in such an experiment, subjects are able to achieve a high level of performance only as long as 
the WM system can rely on transient storage mechanisms, i.e., as long as the stimuli appear with a predictable timing. In contrast, introducing unpredictability in the timing of the stimuli should significantly decrease the performance of the subjects as there are no LTM attractor states available to support the WM operation. This should change, however, if the experiment is repeated several times with the same set of stimuli. The repeated presentation of the same stimuli should trigger the formation of respective representations in LTM which may then support the dynamics in the WM system. The performance of the subjects in the n-back task with unpredictable stimulus timing should therefore eventually reach the same level of performance as achieved with predictable stimulus timing.

In summary, the neuronal memory models developed and analyzed in this thesis offer various possibilities for future works. Besides of different research questions regarding the individual models, their combination might allow to model a complex cognitive-motor WM dual-task as well as closed-loop scenarios requiring the transformation of a sequence of stimuli into specific locomotion behaviors. Finally, our results also yield nontrivial experimental predictions that may be verified in a modified version of the n-back task.

\section{Summary}

In this thesis, we show that reliable WM operation requires an interplay of both transient dynamics and attractor states and that these attractor states may be formed in a self-organized way in other neuronal memory systems which interact with the neuronal system implementing WM. We analyze the neuronal dynamics required to solve a WM task with unpredictable temporal structure and show that only by exploiting both transient dynamics and attractor states, a neuronal system is able to solve this task. Furthermore, we present and analyze a new neuronal network model of LTM which, in contrast to earlier models, allows to form CAs and simultaneously allocate these CAs to the correct stimuli in a self-organized way based on the interaction of two elementary plasticity processes. These CAs are input-dependent attractor states and may thus provide the kind of attractor dynamics required by WM to process unreliably timed stimuli. Finally, we develop a new mechanism which is able to adapt the frequencies of general oscillatory systems based on periodic input signals within a wide frequency range faster and more precisely as compared to an existing mechanism. This new mechanism may allow short-lasting transient trajectories in the WM system to evoke long-lasting time-dependent output signals. Although our models of the individual memory system involve several abstractions and simplifications, our studies provide potential explanations for several experimental findings and open questions (e.g. memory maintenance without persistent activity only in WM experiments with predictable temporal structure). At the same time, there remain several open questions regarding the actual interaction of the investigated memory systems. Thus, our results may be the starting point for a more detailed model incorporating all the investigated neuronal memory systems. This will allow to study the emergence of complex behavior out of the interaction of different neuronal memory systems. 



\section{Appendix A}

\section{Supplementary Calculations for the Cell Assembly Model}

\section{A.1 Normalization of the Network Model}

The dynamics of the neurons and synapses in the network model in Chapter 4 is based on the model proposed by Tetzlaff et al., (2013). In contrast to Tetzlaff et al., (2013), however, we use a normalized version of both the neuronal dynamics and the synaptic dynamics. This reduces the number of covariant parameters in the model. Here, we show how to transform the unnormalized model into an equivalent normalized version.

\section{Unnormalized Model}

In the following, we denote all variables of the unnormalized model by a tilde ( $\left.{ }^{\sim}\right)$. In this model, the dynamics of the membrane potential $\tilde{u}_{i}$ of neuron $i\left(i \in\left\{1, \ldots, N^{\text {rec }}\right\}\right)$ in the recurrent area is given by the following equation:

$$
\frac{\mathrm{d} \tilde{u}_{i}}{\mathrm{~d} t}=-\frac{\tilde{u}_{i}}{\tilde{\tau}}+\tilde{R}\left(\sum_{j \in C_{i}^{\text {rec }}} \tilde{w}_{i j}^{\text {rec }} \tilde{F}_{j}+\sum_{k \in C_{i}^{\text {fff }}} \tilde{w}_{i k}^{\text {ff }} \tilde{I}_{k}+\tilde{w}_{\text {out }}^{\text {inh }} \tilde{F}^{\text {inh }}\right) .
$$

Here, the time scale $\tilde{\tau}$ defines the decay rate of the membrane potential. The membrane resistance $\tilde{R}$ scales the influence of the incoming signals on the membrane potential. The weights $\tilde{w}_{i j}^{\text {rec }}$ of the recurrent synapses characterize the interaction between neurons in the recurrent area to the inhibitory population. The set $C_{i}^{\text {rec }}$ contains all indexes of neurons $j$ within the recurrent area which transmit synaptic signals to neuron $i$. Accordingly, the weights of the feedforward synapses transmitting signals from the inputs $\tilde{I}_{k}\left(k \in\left\{1, \ldots, N^{\text {in }}\right\}\right)$ to neurons within the recurrent area are given by $\tilde{w}_{i k}^{\text {ff }}$. The set $C_{i}^{\mathrm{ff}}$ contains all indexes $k$ of neurons in the input area which transmit signals to neuron $i$ in the recurrent area. Finally, the weight $\tilde{w}_{\text {out }}^{\text {inh }}$ determines the strengths of the synapses via which the neurons in the recurrent area receive signals from the inhibitory population. The firing rate of the inhibitory population is given by $\tilde{F}^{\text {inh }}$.

In the unnormalized model, the firing rate function of the neurons in the recurrent area is given by

$$
\tilde{F}_{i}=\tilde{\phi}\left(\tilde{u}_{i}\right)=\frac{\tilde{\alpha}}{1+\exp \left(\tilde{\beta}\left(\tilde{\epsilon}-\tilde{u}_{i}\right)\right)} .
$$

In addition to the location $\tilde{\epsilon}$ of the inflection point and the steepness $\tilde{\beta}$, Equation (A.2) also contains a parameter $\tilde{\alpha}$ which determines the maximum firing rate. 

by

The dynamics of the membrane potential $\tilde{u}^{\text {inh }}$ of the inhibitory population is given

$$
\frac{\mathrm{d} \tilde{u}^{\mathrm{inh}}}{\mathrm{d} t}=-\frac{\tilde{u}^{\mathrm{inh}}}{\tilde{\tau}^{\mathrm{inh}}}+\tilde{R}^{\mathrm{inh}} \sum_{i=1}^{N^{\mathrm{rec}}} \tilde{w}_{\mathrm{in}}^{\text {inh }} \tilde{F}_{i}
$$

with the time constant $\tilde{\tau}^{\text {inh }}$, the membrane resistance $\tilde{R}^{\text {inh }}$ and the weight $\tilde{w}_{\text {in }}^{\text {inh }}$ of the synapses transmitting excitatory signals from neurons in the recurrent area. The firing rate $\tilde{F}^{\text {inh }}$ of the inhibitory population is determined by the inhibitory firing rate function:

$$
\tilde{F}^{\text {inh }}=\tilde{\phi}^{\text {inh }}\left(\tilde{u}^{\text {inh }}\right)=\frac{\tilde{\alpha}}{1+\exp \left(\tilde{\beta}^{\text {inh }}\left(\tilde{\epsilon}^{\text {inh }}-\tilde{u}^{\text {inh }}\right)\right)}
$$

with the parameters $\tilde{\beta}^{\text {inh }}$ and $\tilde{\epsilon}^{\text {inh }}$.

According to Tetzlaff et al., (2013), the synaptic plasticity of the recurrent synapse transmitting signals from neuron $j$ to neuron $i$ is described by the following differential equation:

$$
\frac{\mathrm{d} \tilde{w}_{i j}^{\mathrm{rec}}}{\mathrm{d} t}=\tilde{\mu}^{\mathrm{rec}}\left(\tilde{F}_{i} \tilde{F}_{j}+\left(\tilde{\kappa}^{\mathrm{rec}}\right)^{-1}\left(\tilde{F}^{\mathrm{T}}-\tilde{F}_{i}\right)\left(\tilde{w}_{i j}^{\mathrm{rec}}\right)^{2}\right) .
$$

Here, $\tilde{\mu}^{\text {rec }}$ is the learning rate of the recurrent synapses. The parameter $\tilde{\kappa}^{\text {rec }}$ is supposed to describe the ratio of the time scales of Hebbian plasticity and synaptic scaling. Larger values of $\tilde{\kappa}^{\text {rec }}$ reduce the contributions of the synaptic scaling term. The target firing rate $\tilde{F}^{\mathrm{T}}$ defines the set point of the synaptic scaling process. The plasticity of the feedforward synapses is described by similar dynamics:

$$
\frac{\mathrm{d} \tilde{w}_{i k}^{\mathrm{ff}}}{\mathrm{d} t}=\tilde{\mu}^{\mathrm{ff}}\left(\tilde{F}_{i} \tilde{F}_{k}+\left(\tilde{\kappa}^{\mathrm{ff}}\right)^{-1}\left(\tilde{F}^{\mathrm{T}}-\tilde{F}_{i}\right)\left(\tilde{w}_{i k}^{\mathrm{ff}}\right)^{2}\right)
$$

with a learning rate $\tilde{\mu}^{\mathrm{ff}}$ and time scale ratio $\tilde{\kappa}^{\mathrm{ff}}$.

\section{Normalization}

In order to reduce the number of covariant parameters in the network model, in this thesis, we use normalized versions of the neuronal and synaptic dynamics described above. Here, we show how to transform a given parameter configuration of the unnormalized model into a parameter configuration of the normalized one. We thus prove that every solution of the unnormalized model can be reduced to a solution of the normalized model. Starting from a parameterization of the unnormalized model, we introduce rescaled variables $u_{i}$ and $u^{\text {inh }}$ for the excitatory and inhibitory membrane potentials:

$$
\begin{aligned}
u_{i} & =\frac{1}{\tilde{\tau} \tilde{R} \tilde{\alpha}^{2}} \sqrt{\frac{\tilde{\alpha}-\tilde{F}^{\mathrm{T}}}{\tilde{\kappa}^{\mathrm{rec}}}} \tilde{u}_{i} \\
u^{\mathrm{inh}} & =\frac{1}{\tilde{\tau}^{\mathrm{inh}} \tilde{\alpha} \tilde{R}^{\mathrm{inh}}} \tilde{u}^{\mathrm{inh}} .
\end{aligned}
$$


We also introduce rescaled versions $w_{i j}^{\mathrm{rec}}$ and $w_{i k}^{\mathrm{ff}}$ of the weights of the recurrent and feedforward synapses:

$$
\begin{aligned}
w_{i j}^{\mathrm{rec}} & =\frac{1}{\tilde{\alpha}} \sqrt{\frac{\tilde{\alpha}-\tilde{F}^{\mathrm{T}}}{\tilde{\kappa}^{\mathrm{rec}}}} \tilde{w}_{i j}^{\mathrm{rec}} \\
w_{i k}^{\mathrm{ff}} & =\frac{1}{\tilde{\alpha}} \sqrt{\frac{\tilde{\alpha}-\tilde{F}^{\mathrm{T}}}{\tilde{\kappa}^{\mathrm{rec}}}} \tilde{w}_{i k}^{\mathrm{ff}} .
\end{aligned}
$$

Furthermore, we define scaled firing rates $F_{i}$ of the excitatory neurons and $F^{\text {inh }}$ of the inhibitory population:

$$
\begin{aligned}
F_{i} & =\phi\left(u_{i}\right)=\frac{\tilde{F}_{i}}{\tilde{\alpha}}=\frac{\tilde{\phi}\left(\tilde{u}_{i}\right)}{\tilde{\alpha}} \\
F^{\text {inh }} & =\phi^{\text {inh }}\left(u^{\text {inh }}\right)=\frac{\tilde{F}^{\text {inh }}}{\tilde{\alpha}}=\frac{\tilde{\phi}^{\text {inh }}\left(\tilde{u}^{\text {inh }}\right)}{\tilde{\alpha}} .
\end{aligned}
$$

Finally, we also introduce a set of scaled parameter values:

$$
\begin{aligned}
& \tau=\tilde{\tau} \\
& \tau^{\mathrm{inh}}=\tilde{\tau}^{\mathrm{inh}} \\
& \tau^{\mathrm{rec}}=\tilde{\mu}^{\mathrm{rec}} \tilde{\alpha} \sqrt{\frac{\tilde{\alpha}-\tilde{F}^{\mathrm{T}}}{\tilde{\kappa}^{\mathrm{rec}}}} \\
& \tau^{\mathrm{ff}}=\tilde{\mu}^{\mathrm{ff}} \tilde{\alpha} \sqrt{\frac{\tilde{\alpha}-\tilde{F}^{\mathrm{T}}}{\tilde{\kappa}^{\mathrm{ff}}}} \\
& w_{\text {out }}^{\text {inh }}=\sqrt{\frac{\tilde{\alpha}-\tilde{F}^{T}}{\tilde{\kappa}^{\text {rec }}}} \frac{\tilde{w}_{\text {out }}^{\text {inh }}}{\tilde{\alpha}} \\
& w_{\text {in }}^{\text {inh }}=\tilde{w}_{\text {in }}^{\text {inh }} \\
& \beta=\tilde{\tau} \tilde{R} \tilde{\alpha}^{2} \tilde{\beta} \sqrt{\frac{\tilde{\kappa}^{\mathrm{rec}}}{\tilde{\alpha}-\tilde{F}^{\mathrm{T}}}} \\
& \epsilon=\frac{\tilde{\epsilon}}{\tilde{\tau} \tilde{R} \tilde{\alpha}^{2}} \sqrt{\frac{\tilde{\alpha}-\tilde{F}^{\mathrm{T}}}{\tilde{\kappa}^{\mathrm{rec}}}} \\
& \beta^{\text {inh }}=\tilde{\tau}^{\text {inh }} \tilde{R}^{\text {inh }} \tilde{\alpha} \tilde{\beta}^{\text {inh }} \\
& \epsilon^{\mathrm{inh}}=\frac{\tilde{\epsilon}^{\mathrm{inh}}}{\tilde{\tau}^{\mathrm{inh}} \tilde{R}^{\mathrm{inh}} \tilde{\alpha}} \\
& I_{k}=\frac{\tilde{I}_{k}}{\tilde{\alpha}} \\
& F^{\mathrm{T}}=\frac{\tilde{F}^{\mathrm{T}}}{\tilde{\alpha}}
\end{aligned}
$$

In the following, we show that rescaling the unnormalized network model according to the stated rules leads to the normalized version of the model used in Chapter 4 .

\section{Dynamics of the Excitatory Membrane Potentials}

We obtain the dynamics of the scaled membrane potential $u_{i}$ by inserting its definition in Equation (A.1). We simplify the resulting expression by using the scaled variables 
and parameters defined above:

$$
\begin{aligned}
& \frac{\mathrm{d} u_{i}}{\mathrm{~d} t}=\frac{1}{\tilde{\tau} \tilde{R} \tilde{\alpha}^{2}} \sqrt{\frac{\tilde{\alpha}-\tilde{F}^{\mathrm{T}}}{\tilde{\kappa}^{\mathrm{rec}}}} \frac{\mathrm{d} \tilde{u}_{i}}{\mathrm{~d} t} \\
& =-\frac{1}{\tilde{\tau}} \underbrace{\frac{1}{\tilde{\tau} \tilde{R} \tilde{\alpha}^{2}} \sqrt{\frac{\tilde{\alpha}-\tilde{F}^{\mathrm{T}}}{\tilde{\kappa}^{\mathrm{rec}}}} \tilde{u}_{i}}_{=u_{i}}+\frac{1}{\tilde{\tau}} \sum_{j \in C_{i}^{\text {rec }}} \underbrace{\frac{\tilde{w}_{i j}^{\text {rec }}}{\tilde{\alpha}} \sqrt{\frac{\tilde{\alpha}-\tilde{F}^{\mathrm{T}}}{\tilde{\kappa}^{\mathrm{rec}}}}}_{=w_{i j}^{\text {rec }}} \underbrace{\frac{\tilde{F}_{j}}{\tilde{\alpha}}}_{=F_{j}} \\
& +\frac{1}{\tilde{\tau}} \underbrace{\sqrt{\frac{\tilde{\kappa}^{\mathrm{ff}}}{\tilde{\kappa}^{\mathrm{rec}}}}}_{=s} \sum_{k \in C_{i}^{\mathrm{ff}}} \frac{\tilde{w}_{i k}^{\mathrm{ff}}}{\frac{\tilde{\alpha}}{\frac{\tilde{\alpha}-\tilde{F}^{\mathrm{T}}}{\tilde{\kappa}^{\mathrm{rec}}}}} \underbrace{\frac{\tilde{I}_{k}}{\tilde{\alpha}}}_{=w_{i k}^{\mathrm{ff}}}+\underbrace{\sqrt{\frac{\tilde{\alpha}-\tilde{F}^{\mathrm{T}}}{\tilde{\kappa}^{\mathrm{rec}}}} \frac{\tilde{w}_{\mathrm{out}}^{\mathrm{inh}}}{\tilde{\alpha}}}_{=I^{k}} \underbrace{\frac{\tilde{F}^{\mathrm{inh}}}{\tilde{\alpha}}}_{=w_{\text {out }}^{\text {inh }}} \\
& =\frac{1}{\tau}\left(-u_{i}+\sum_{j \in C_{i}^{\text {rec }}} w_{i j}^{\mathrm{rec}} F_{j}+s \sum_{k \in C_{i}^{\mathrm{ff}}} w_{i k}^{\mathrm{ff}} I^{k}+w_{\mathrm{out}}^{\mathrm{inh}} F^{\mathrm{inh}}\right) \text {. }
\end{aligned}
$$

Here, we introduced a correction factor $s=\sqrt{\tilde{\kappa}^{\mathrm{ff}} / \tilde{\kappa}^{\mathrm{rec}}}$. In the case of $\tilde{\kappa}^{\mathrm{ff}}=\tilde{\kappa}^{\text {rec }}$, we have $s=1$ and the final expression in Equation (A.19) directly describes the dynamics of the membrane potential of excitatory neurons in Chapter 4 (compare Equation 4.1). If $\tilde{\kappa}^{\text {ff }} \neq \tilde{\kappa}^{\text {rec }}$, the different maximum strengths of recurrent and feedforward synapses result in a correction factor $s \neq 1$. Instead of explicitly including $s$ in Equation (A.19), however, we adjust the number of incoming recurrent and feedforward synapses per neuron. If we have $s=2$, for instance, we double the number of incoming feedforward synapses per neuron in the recurrent area.

\section{Dynamics of the Inhibitory Membrane Potential}

From Equation (A.3), we obtain the dynamics of the scaled membrane potential $u^{\text {inh }}$ as follows:

$$
\begin{aligned}
\frac{\mathrm{d} u^{\mathrm{inh}}}{\mathrm{d} t} & =\frac{1}{\tilde{\tau}^{\mathrm{inh}} \tilde{\alpha} \tilde{R}^{\mathrm{inh}}} \frac{\mathrm{d} \tilde{u}^{\mathrm{inh}}}{\mathrm{d} t} \\
& =\frac{1}{\tilde{\tau}^{\mathrm{inh}}}(-\underbrace{\frac{\tilde{u}^{\mathrm{inh}}}{\tilde{\tau}^{\mathrm{inh}} \tilde{\alpha} \tilde{R}^{\mathrm{inh}}}}_{u^{\mathrm{inh}}}+\sum_{i=1}^{N^{\mathrm{rec}}} \tilde{w}_{\mathrm{in}}^{\mathrm{inh}} \underbrace{\frac{\tilde{F}_{i}}{\tilde{\alpha}}}_{F_{i}}) \\
& =\frac{1}{\tau^{\mathrm{inh}}}\left(-u^{\mathrm{inh}}+\sum_{i=1}^{\mathrm{N}^{\mathrm{rec}}} w_{\mathrm{in}}^{\mathrm{inh}} F_{i}\right) .
\end{aligned}
$$

This expression corresponds to the dynamics of the inhibitory membrane potential in Equation (4.3).

\section{Excitatory Firing Rate Function}

We rearrange Equation (A.7) to obtain $\tilde{u}_{i}$ as a function of $u_{i}$. Inserting this expression into the firing rate function $\tilde{\phi}$ in Equation (A.2) yields the excitatory firing rate 
function $\phi$ of the normalized model:

$$
\begin{aligned}
\phi\left(u_{i}\right) & =\frac{1}{\tilde{\alpha}} \tilde{\phi}\left(\tilde{\tau} \tilde{R} \tilde{\alpha}^{2} \sqrt{\frac{\tilde{\kappa}^{\mathrm{rec}}}{\tilde{\alpha}-\tilde{F}^{\mathrm{T}}}} u_{i}\right) \\
& =\frac{1}{1+\exp (\underbrace{\tilde{\tau} \tilde{R} \tilde{\alpha}^{2} \sqrt{\frac{\tilde{\kappa}^{\mathrm{rec}}}{\tilde{\alpha}-\tilde{F}^{\mathrm{T}}}} \tilde{\beta}}_{\beta}(\underbrace{\frac{\tilde{\epsilon}}{\tilde{\tau} \tilde{R} \tilde{\alpha}^{2}} \sqrt{\frac{\tilde{\alpha}-\tilde{F}^{\mathrm{T}}}{\tilde{\kappa}^{\mathrm{rec}}}}}_{\epsilon}-u_{i}))} \\
& =\frac{1}{1+\exp \left(\beta\left(\epsilon-u_{i}\right)\right)} .
\end{aligned}
$$

This is identical to the firing rate function in Equation (4.2).

\section{Inhibitory Firing Rate Function}

Rearranging Equation (A.8) yields $\tilde{u}^{\text {inh }}$ as a function of $u^{\text {inh }}$. This allows us to obtain the firing rate function $\phi^{\text {inh }}$ of the inhibitory population by inserting this expression into the function $\tilde{\phi}^{\text {inh }}$ in Equation (A.4):

$$
\begin{aligned}
\phi\left(u^{\mathrm{inh}}\right) & =\frac{1}{\tilde{\alpha}} \tilde{\phi}^{\mathrm{inh}}\left(\tilde{\tau}^{\mathrm{inh}} \tilde{R}^{\mathrm{inh}} \tilde{\alpha} u^{\mathrm{inh}}\right) \\
& =\frac{1}{1+\exp (\underbrace{\tilde{\tau}^{\operatorname{inh}} \tilde{R}^{\operatorname{inh}} \tilde{\alpha} \tilde{\beta}^{\text {inh }}}_{\beta^{\text {inh }}}(\underbrace{\frac{\tilde{\epsilon}^{\text {inh }}}{\tilde{\tau}^{\text {inh }} \tilde{R}^{\text {inh }} \tilde{\alpha}}}_{\epsilon^{\text {inh }}}-u^{\text {inh }}))} \\
& =\frac{1}{1+\exp \left(\beta^{\text {inh }}\left(\epsilon^{\text {inh }}-u^{\text {inh }}\right)\right)} .
\end{aligned}
$$

The final expression in Equation (A.22) corresponds to the firing rate function of the inhibitory population in Equation (4.4).

\section{Plasticity of Recurrent Synapses}

By expressing the dynamics of the weights of the recurrent synapses in Equation (A.5) in terms of the rescaled variables we obtain:

$$
\begin{aligned}
& \frac{\mathrm{d} w_{i j}^{\mathrm{rec}}}{\mathrm{d} t}=\frac{1}{\tilde{\alpha}} \sqrt{\frac{\tilde{\alpha}-\tilde{F}^{\mathrm{T}}}{\tilde{\kappa}^{\mathrm{rec}}}} \frac{\mathrm{d} \tilde{w}_{i j}^{\mathrm{rec}}}{\mathrm{d} t}
\end{aligned}
$$

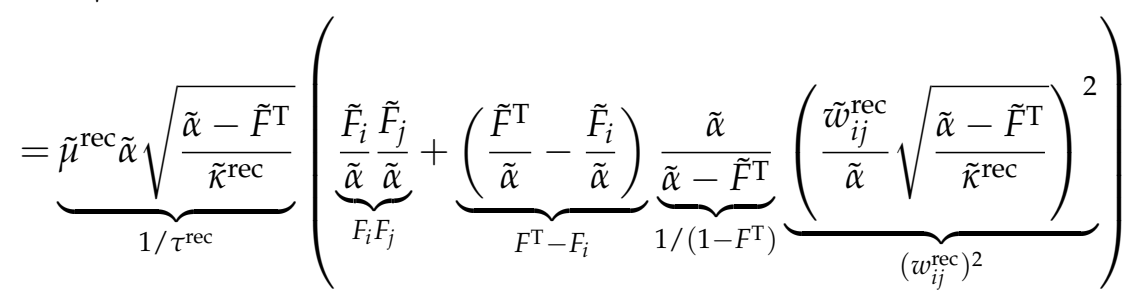

$$
\begin{aligned}
& =\frac{1}{\tau^{\mathrm{rec}}}\left(F_{i} F_{j}+\frac{F^{\mathrm{T}}-F_{i}}{1-F^{\mathrm{T}}}\left(w_{i j}^{\mathrm{rec}}\right)^{2}\right) \text {. }
\end{aligned}
$$

This is the plasticity rule of the recurrent synapses as stated in Equation (4.5). 


\section{Plasticity of Feedforward Synapses}

Finally, we transform the plasticity rule of the feedforward synapses in Equation (A.6) into the equivalent expression for the rescaled synaptic weight $w_{i k}^{\mathrm{ff}}$ :

$$
\begin{aligned}
\frac{\mathrm{d} w_{i k}^{\mathrm{ff}}}{\mathrm{d} t} & =\frac{1}{\tilde{\alpha}} \sqrt{\frac{\tilde{\alpha}-\tilde{F}^{\mathrm{T}}}{\tilde{\kappa}^{\mathrm{ff}}}} \frac{\mathrm{d} \tilde{w}_{i k}^{\mathrm{ff}} \mathrm{d} t}{} \\
& =\underbrace{\tilde{\mu}^{\mathrm{ff}} \tilde{\alpha} \sqrt{\frac{\tilde{\alpha}-\tilde{F}^{\mathrm{T}}}{\tilde{\mathcal{K}}^{\mathrm{ff}}}}}_{1 / \tau^{\mathrm{ff}}}(\underbrace{\frac{\tilde{F}_{i}}{\tilde{\alpha}} \frac{\tilde{I}_{k}}{\tilde{\alpha}}}_{F_{i} I_{k}}+\underbrace{\left(\frac{\tilde{F}^{\mathrm{T}}}{\tilde{\alpha}}-\frac{\tilde{F}_{i}}{\tilde{\alpha}}\right)}_{F^{\mathrm{T}}-F_{i}} \underbrace{\frac{\tilde{\alpha}}{\tilde{\alpha}-\tilde{F}^{\mathrm{T}}}}_{1 /\left(1-F^{\mathrm{T}}\right)} \underbrace{\left(\frac{\tilde{w} \tilde{w}_{i k}^{\mathrm{ff}}}{\tilde{\alpha}} \sqrt{\left.\frac{\tilde{\alpha}-\tilde{F}^{\mathrm{T}}}{\tilde{\mathcal{K}}^{\mathrm{ff}}}\right)^{2}}\right)}_{\left(w_{i k}^{\mathrm{ff}}\right)^{2}} \\
& =\frac{1}{\tau^{\mathrm{ff}}}\left(F_{i} I_{k}+\frac{F^{\mathrm{T}}-F_{i}}{1-F^{\mathrm{T}}}\left(w_{i k}^{\mathrm{ff}}\right)^{2}\right) .
\end{aligned}
$$

Note that this expression is identical to the plasticity rule stated in Equation (4.6).

Thus, we have shown that the rescaled variables and parameters in Equations (A.7-A.18) transform any parametrization of the unnormalized network model into an equivalent parametrization of the normalized network model. In other words, studying the normalized network model suffices to capture the complete dynamics of the unnormalized model.

\section{A.2 Jacobi Matrix of the Population Model}

In order to determine the stability of the equilibria of the population model in Section 4.3, we numerically calculate the eigenvalues of the Jacobi matrix of the model at the respective equilibria. In the following, we state all nonzero terms of the Jacobi matrix $(i \in\{1,2\}$ and $k \in\{\mathrm{A}, \mathrm{B}\})$ :

$$
\begin{aligned}
& \frac{\partial \dot{\bar{u}}_{i}}{\partial \bar{u}_{i}}=\frac{1}{\tau}\left(-1+\bar{n}_{++}^{\text {rec }} \bar{w}_{i}^{\text {rec }} \frac{\partial \bar{F}_{i}}{\partial \bar{u}_{i}}\right) \quad \frac{\partial \dot{\bar{u}}_{i}}{\partial u^{\text {inh }}}=\frac{w_{\text {out }}^{\text {inh }}}{\tau} \frac{\partial F^{\text {inh }}}{\partial u^{\text {inh }}} \\
& \frac{\partial \dot{\bar{u}}_{i}}{\partial \bar{w}_{i}^{\mathrm{rec}}}=\frac{\bar{n}_{++}^{\mathrm{rec}} \bar{F}_{i}}{\tau} \quad \frac{\partial \dot{\bar{u}}_{i}}{\partial \bar{w}_{i}^{\mathrm{ff}}}=\frac{\bar{n}_{++}^{\mathrm{ff}} \bar{I}_{k}}{\tau} \\
& \frac{\partial \dot{\bar{u}}^{\text {inh }}}{\partial \bar{u}_{i}}=\frac{w_{\text {in }}^{\text {inh }} N_{\text {pop }}}{\tau^{\text {inh }}} \frac{\partial \bar{F}_{i}}{\partial \bar{u}_{i}} \\
& \frac{\partial \dot{\bar{u}}^{\text {inh }}}{\partial \bar{u}^{\text {inh }}}=-\frac{1}{\tau^{\text {inh }}} \\
& \frac{\partial \dot{\bar{w}}_{i}^{\text {rec }}}{\partial \bar{u}_{i}}=\frac{2 \bar{F}_{i}-\left(\bar{w}_{i}^{\text {rec }}\right)^{2}}{\tau^{\text {rec }}} \frac{\partial \bar{F}_{i}}{\partial \bar{u}_{i}} \\
& \frac{\partial \dot{\bar{w}}_{i}^{\mathrm{rec}}}{\partial \bar{w}_{i}^{\mathrm{rec}}}=\frac{2 \bar{w}_{i}^{\mathrm{rec}}}{\tau^{\mathrm{rec}}} \frac{F_{\mathrm{T}}-\bar{F}_{i}}{1-F_{\mathrm{T}}} \\
& \frac{\partial \dot{\bar{w}}_{i}^{\mathrm{ff}}}{\partial \bar{u}_{i}}=\frac{1}{\tau^{\mathrm{ff}}}\left(\bar{I}_{i}-\frac{\left(\bar{w}_{i}^{\mathrm{ff}}\right)^{2}}{1-F_{\mathrm{T}}}\right) \frac{\partial \bar{F}_{i}}{\partial \bar{u}_{i}} \\
& \frac{\partial \dot{\bar{w}}_{i}^{\mathrm{ff}}}{\partial \bar{w}_{i}^{\mathrm{ff}}}=\frac{2 \bar{w}_{i}^{\mathrm{ff}}}{\tau^{\mathrm{ff}}} \frac{F_{\mathrm{T}}-\bar{F}_{i}}{1-F_{\mathrm{T}}}
\end{aligned}
$$

where

$$
\frac{\partial \bar{F}_{i}}{\partial \bar{u}_{i}}=\beta \bar{F}_{i}\left(1-\bar{F}_{i}\right) \quad \text { and } \quad \frac{\partial \bar{F}^{\text {inh }}}{\partial \bar{u}^{\text {inh }}}=\beta^{\text {inh }} \bar{F}^{\text {inh }}\left(1-\bar{F}^{\text {inh }}\right) .
$$




\section{A.3 Maximum Input Amplitude for Cell Assembly Forma- tion}

In Section 4.3.2, we state that there is a maximum input amplitude $\bar{I}_{\mathrm{A}}$ of a stimulus A which allows for a stable formation of CAs in the network model. For higher amplitudes, the signals transmitted from the inhibitory population do not suffice to balance the recurrent and feedforward excitatory signals in the recurrent area. As a consequence, the recruitment of additional neurons in the recurrent area continues until eventually all neurons in the recurrent area are active.

The derivation of the maximum input amplitude that can be balanced by the inhibitory population is similar to the derivation of the number of neurons in a CA in Section 4.2.2. We assume that a stimulus $\mathrm{A}$ activates $N_{+}^{\text {in }}$ neurons in the input area such that these neurons fire at a rate of $I_{\mathrm{A}}$. Furthermore, we assume that this stimulus has lead to the formation of a CA in the recurrent area. As in Section 4.2.2, we investigate the equilibrium potential of a neuron $i$ in the recurrent area which is not active and located directly next to the active CA neurons (Figure $4.5 \mathrm{a}$ ). This neuron receives recurrent synaptic signals from $n_{i,+}^{\text {rec }}$ active neurons in the recurrent area. It also receives and $n_{i,+}^{\mathrm{ff}}$ feedforward signals from active neurons in the input area.

In the equilibrium, the weights of the recurrent synapses from active neurons are at their maximum value of 1 (compare Equation 4.20). The equilibrium weights of the feedforward synapses transmitting signals from active neurons in the input area are approximated by $w_{i,+}^{\mathrm{ff}, *} \approx \sqrt{I_{\mathrm{A}}}$ (compare Equation (4.19)). This approximation is valid for low values of the target firing rate $F_{\mathrm{T}}$ of the synaptic scaling term. For $F_{\mathrm{T}}=0$ as used in this thesis, $w_{i,+}^{\mathrm{ff}, *}=\sqrt{I_{\mathrm{A}}}$ is exact. Based on these assumptions, similar to Equation (4.11), we express the equilibrium membrane potential of neuron $i$ as a function of $I_{\mathrm{A}}$ :

$$
\begin{aligned}
& u_{i}^{*}=\sum_{j \in C_{i}^{\text {rec }}} w_{i j}^{\mathrm{rec}, *} F_{j}^{*}+\sum_{k \in C_{i}^{\mathrm{ff}}} w_{i k}^{\mathrm{ff}, *} I_{k}+w_{\mathrm{out}}^{\mathrm{inh}} F^{\mathrm{inh}, *} \\
& =\underbrace{\sum_{\substack{j \in C_{i}^{\text {rec }} \\
F_{j}^{*}>0.5}} w_{i j}^{\mathrm{rec}, *} F_{j}^{*}}_{\approx n_{i,+}^{\text {rec }}}+\underbrace{\sum_{\substack{j \in C_{i}^{\text {rec }} \\
F_{j}^{*} \leq 0.5}} w_{i j}^{\mathrm{rec}, *} F_{j}^{*}}_{\approx 0}+\underbrace{\sum_{\substack{k \in C_{i}^{\mathrm{ff}} \\
I_{k}=I_{\mathrm{A}}}} w_{i k}^{\mathrm{ff}, *} I_{k}}_{=n_{i,+}^{\mathrm{ff}} \sqrt{I_{\mathrm{A}}} I_{\mathrm{A}}}+\underbrace{\sum_{\substack{k \in C_{i}^{\mathrm{ff}} \\
I_{k}=0}} w_{i k}^{\mathrm{ff}, *} I_{k}}_{\approx 0}+w_{\mathrm{out}}^{\mathrm{inh}} F^{\mathrm{inh}, * *} \\
& \approx n_{i,+}^{\mathrm{rec}}+n_{i,+}^{\mathrm{ff}} I_{\mathrm{A}}^{\frac{3}{2}}+w_{\mathrm{out}}^{\mathrm{inh}} F^{\mathrm{inh}, *} .
\end{aligned}
$$

In order to limit the recruitment of neurons in the recurrent area into the $\mathrm{CA}$, the signals received by a neuron $i$ must not allow this neuron to become active. In other words, the equilibrium potential $u_{i}^{*}$ of neuron $i$ must be lower than the potential $\epsilon$ required to reach the inflection point of the firing rate function: $u_{i}^{*}<\epsilon$. In particular, this must be true if the equilibrium activity of the inhibitory population is given by its maximum firing rate $F^{\mathrm{inh}, *}=1$. Therefore, we require

$$
u_{i}^{*}=n_{i,+}^{\mathrm{rec}}+n_{i,+}^{\mathrm{ff}} I_{\mathrm{A}}^{\frac{3}{2}}+w_{\mathrm{out}}^{\mathrm{inh}}<\epsilon .
$$


Isolating $I_{\mathrm{A}}$ in this inequality yields the expression for the maximum input amplitude $I_{\mathrm{A}}^{\max }$ which allows for stable CA formation:

$$
I_{\mathrm{A}}<\left(\frac{\epsilon-w_{\mathrm{inh}}^{\mathrm{out}}-n_{i,+}^{\mathrm{rec}}}{n_{i,+}^{\mathrm{ff}}}\right)^{\frac{2}{3}}=I_{\mathrm{A}}^{\max } .
$$

According to the parametrization of the network model in Chapter 4, we have $\epsilon=12$ and $w_{\text {out }}^{\text {inh }}=-20$ (Table 4.1). Furthermore, based on the recurrent interaction radius $r^{\text {rec }}=3$, a neuron $i$ in the described position directly next to a CA receives synaptic signals from $n_{i,+}^{\text {rec }}=11$ active neurons in the recurrent area (Figure $4.5 \mathrm{a}$ ). The expected number of synaptic signals from active neurons in the input area is $n_{i,+}^{\mathrm{ff}}=N^{\mathrm{in}} n^{\mathrm{ff}} / N^{\mathrm{inh}}=50 \cdot 25 / 100=12.5$. Thus, we obtain a maximum input amplitude of $I_{\mathrm{A}}^{\max } \approx 1.41$. 


\section{Appendix B}

\section{Frequency-Curve of the Van der Pol Oscillator}

As stated in Equation (5.3), the Van der Pol oscillator is given by the following system of equations for the state variables $x$ and $y$ :

$$
\begin{aligned}
& \dot{x}(t)=y(t) \\
& \dot{y}(t)=\mu\left(1-x(t)^{2}\right) y(t)-\theta^{2} x(t) .
\end{aligned}
$$

The parameter $\mu>0$ determines the degree of nonlinearity of the limit cycle (Figure B.1 a). For small values of $\mu$, the Van der Pol oscillator is almost harmonic. Thus, the limit cycle in the $x-y$-space is close to a circle. For larger values of $\mu$, the shape of the limit cycle is more complex. While, its expansion in the direction of the variable $x$ is constant, $y$ reaches, according to amount, larger values for larger values of $\mu$. The variable $\theta$ determines the intrinsic frequency $v$ of the oscillations. There is no analytical expression for the function $v(\theta)$ available. In addition, this function also depends of the variable $\mu$ (Figure B.1 b).

In order to obtain the oscillation frequency of the Van der Pol oscillator for a given $(\mu, \theta)$-frequency pair, we numerically integrate the dynamical system in Equation (B.1) for a duration $t_{\mathrm{FFT}}=1000$. We sample the variable $x$ at time intervals of $\Delta t_{\mathrm{FFT}}=0.025$ and use the SciPy software package (Jones et al., 2001) to perform a fast Fourier transform (Cooley and Tukey, 1965) on the sampled data. The frequency component with the highest magnitude identifies the intrinsic frequency $v$ of the system. Note that the precision of this frequency depends on the length of the
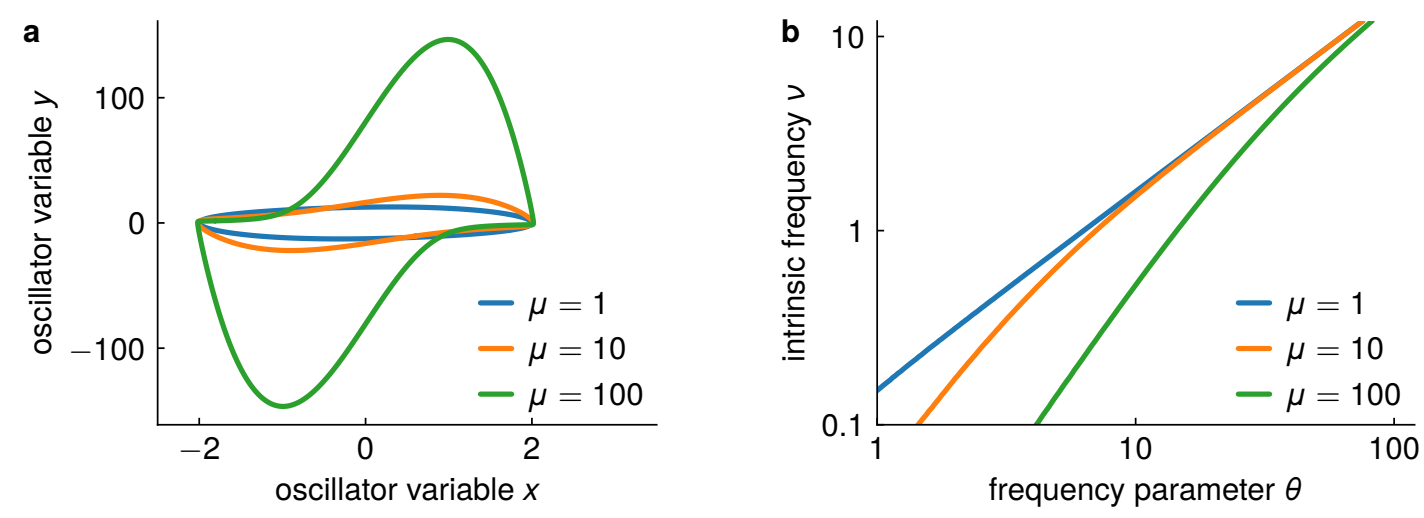

Figure B.1: Limit cycle and frequency curve of the Van der Pol oscillator. (a) The shape of the limit cycle in the $x-y$-space depends on the parameter $\mu$ of the oscillator. For low values of $\mu$, the limit cycle approaches a circle (dashed line). For hight values of $\mu$, the shape of the limit cycle is more complex and reaches large positive and negative values of $y$. (b) The relation between the frequency $v$ 
sampled time interval $t_{\mathrm{FFT}}$. The smallest distinguishable frequency difference is given by $\Delta v=1 / t_{\mathrm{FFT}}$. The maximum unambiguously detectable frequency is given by the Nyquist frequency $v_{\text {nyquist }}=1 /\left(2 \Delta t_{\mathrm{FFT}}\right)$.

When transforming a frequency $v$ into the corresponding parameter $\theta$, we use a binary search algorithm. We start with a minimum parameter bound $\theta_{\min }$ that corresponds to a frequency $v_{\min }$ and a maximum parameter bound $\theta_{\max }$ that corresponds to a frequency $v_{\max }$. Accordingly, in the beginning, we have $\theta_{\min }<\theta<\theta_{\max }$ and $v_{\min }<v<v_{\max }$. We then evaluate the frequency $v_{\text {test }}$ of the parameter $\theta_{\text {test }}=0.5\left(\theta_{\min }+\theta_{\max }\right)$ using the fast Fourier transform as described above. If $v_{\text {test }}<v$, we update the value of $\theta_{\min }: \theta_{\text {min }} \leftarrow \theta_{\text {test }}$. If, in contrast, $v_{\text {test }}>v$, we update the value of $\theta_{\max }: \theta_{\max } \leftarrow \theta_{\text {test }}$. Thus, we still have $\theta_{\min }<\theta<\theta_{\max }$ but halved the interval $\theta_{\max }-\theta_{\max }$. We repeat this procedure until we have reached the desired level of precision, i.e., until the difference $v_{\max }-v_{\min }$ is sufficiently small. For our analyses, we require $v_{\max }-v_{\min }<10^{-4}$. Once this condition is met, we set $\theta=0.5\left(\theta_{\min }+\theta_{\max }\right)$. 


\section{Bibliography}

Abbott, L. F. and Nelson, S. B. (2000). Synaptic plasticity: taming the beast.. Nat. Neurosci. 3 Suppl, pp. 1178-83. DOI: 10.1038/81453.

Ahlborn, B. K. and Blake, R. W. (2002). Walking and running at resonance.. Zoology (Jena) 105, pp. 165-74. DOI: 10.1078/0944-2006-00057.

Andronov, A. A., Leontovich, E. A., Gordon, I. I., and Maier, A. G. (1971). Theory of Bifurcations of Dynamical Systems on a Plane. Translated. Jerusalem: Israel Program for Scientific Translations.

Auth, J. M., Nachstedt, T., and Tetzlaff, C. (2017). The interplay of synaptic plasticity and scaling enables the self-organized allocation of multiple memory representations. submmitted.

Baddeley, A. D. (2000). The episodic buffer: A new component of working memory?. Trends Cogn. Sci. 4, pp. 417-423. DOI: 10.1016/S1364-6613(00)01538-2.

Baddeley, A. D. (2003). Working memory: looking back and looking forward.. Nat. Rev. Neurosci. 4, pp. 829-839. DOI: 10.1038/nrn1201.

Baddeley, A. D. (2012). Working memory: theories, models, and controversies.. Annu. Rev. Psychol. 63, pp. 1-29. DOI: 10.1146/annurev-psych-120710-100422.

Baddeley, A. D. and Hitch, G. (1974). "Working Memory". The psychology of learning and motivation: Advances in research and theory. Ed. by G. H. Bower. New York: Academic Press, pp. 47-89.

Baddeley, A. D., Papagno, C., and Vallar, G. (1988). When long-term learning depends on short-term storage. J. Mem. Lang. 27, pp. 586-595. DOI: 10.1016/0749-596X (88) 90028-9.

Bakker, A., Kirwan, C. B., Miller, M., and Stark, C. E. L. (2008). Pattern separation in the human hippocampal CA3 and dentate gyrus. Science 319, pp. 1640-1642. DOI: 10.1126/science. 1152882.

Barak, O., Sussillo, D., Romo, R., Tsodyks, M., and Abbott, L. F. (2013). From fixed points to chaos: three models of delayed discrimination. Prog. Neurobiol. 103, pp. 214-222. DOI: 10.1016/j . pneurobio.2013.02.002.

Barak, O. and Tsodyks, M. (2014). Working models of working memory.. Curr. Opin. Neurobiol. 25, pp. 20-24. DOI: 10.1016/j . conb. 2013.10.008.

Barak, O., Tsodyks, M., and Romo, R. (2010). Neuronal population coding of parametric working memory.. J. Neurosci. 30, pp. 9424-9430. DOI: 10.1523/JNEUROSCI . 1875-10.2010.

Barkai, N. and Leibler, S. (2000). Circadian clocks limited by noise.. Nature 403, pp. 267-8. DOI: $10.1038 / 35002258$.

Barnett, M. W. and Larkman, P. M. (2007). The action potential.. Pract Neurol 7, pp. 192197. DOI: $10.1007 / 978-3-319-26197-3 \backslash \_3$.

Bathellier, B., Ushakova, L., and Rumpel, S. (2012). Discrete neocortical dynamics predict behavioral categorization of sounds.. Neuron 76, pp. 435-449. DOI: 10 . 1016/j.neuron.2012.07.008.

Bennett, M. V. L. and Zukin, R. S. (2004). Electrical Coupling and Neuronal Synchronization in the Mammalian Brain. Neuron 41, pp. 495-511. DOI: 10.1016/S08966273(04)00043-1. 
Bertschinger, N. and Natschläger, T. (2004). Real-time computation at the edge of chaos in recurrent neural networks.. Neural Comput. 16, pp. 1413-36. DOI: 10 . 1162/089976604323057443.

Bick, C. and Rabinovich, M. I. (2009). Dynamical origin of the effective storage capacity in the brain's working memory. Phys. Rev. Lett. 103, p. 218101. DOI: $10.1103 /$ PhysRevLett.103.218101.

Blumenfeld, B., Preminger, S., Sagi, D., and Tsodyks, M. (2006). Dynamics of Memory Representations in Networks with Novelty-Facilitated Synaptic Plasticity. Neuron 52, pp. 383-394. DOI: 10.1016/j.neuron.2006.08.016.

Brown, G. D. A., Preece, T., and Hulme, C. (2000). Oscillator-based memory for serial order.. Psychol. Rev. 107, pp. 127-181. DOI: 10.1037//0033-295X.107.1.127.

Brown, J. (1958). Some tests of the decay theory of immediate memory. Q. J. Exp. Psychol. 10, pp. 12-21. DOI: 10.1080/17470215808416249.

Brown, P. N. and Hindmarsh, A. C. (1989). Reduced storage matrix methods in stiff ODE systems. Appl. Math. Comput. 31, pp. 40-91. DOI: 10. 1016/0096-3003 (89) 90110-0.

Brown, T. G. (1911). The Intrinsic Factors in the Act of Progression in the Mammal. Proc. R. Soc. Lond., B, Biol. Sci. 84, pp. 308-319. DOI: 10.1098/rspb.1911.0077.

Brunel, N. (2016). Is cortical connectivity optimized for storing information?. Nat. Neurosci. 19, pp. 749-755. DOI: 10.1038/nn. 4286.

Buchli, J., Iida, F., and Ijspeert, A. J. (2006a). "Finding Resonance: Adaptive Frequency Oscillators for Dynamic Legged Locomotion". Proceedings of the 2006 IEEE/RSJ International Conference on Intelligent Robots and Systems. Beijing: IEEE, pp. 39033909.

Buchli, J., Righetti, L., and Ijspeert, A. J. (2006b). Engineering entrainment and adaptation in limit cycle systems : From biological inspiration to applications in robotics.. Biol. Cybern. 95, pp. 645-664. DOI: 10.1007/s00422-006-0128-y.

Buchli, J., Righetti, L., and Ijspeert, A. J. (2008). Frequency analysis with coupled nonlinear oscillators. Physica D 237, pp. 1705-1718. DOI: 10.1016/j . physd. 2008. 01.014.

Buonomano, D. V. and Maass, W. (2009). State-dependent computations: spatiotemporal processing in cortical networks.. Nat. Rev. Neurosci. 10, pp. 113-125. DOI: $10.1038 / \mathrm{nrn} 2558$.

Burgess, N. and Hitch, G. (2005). Computational models of working memory: Putting long-term memory into context. Trends Cogn. Sci. 9, pp. 535-541. DOI: 10.1016/j . tics.2005.09.011.

Burgess, N. and Hitch, G. J. (1999). Memory for serial order: A network model of the phonological loop and its timing. Psychol. Rev. 106, pp. 551-581. DOI: $10.1037 /$ 10033-295X.106.3.551.

Burkitt, A. N. (2006). A review of the integrate-and-fire neuron model: I. Homogeneous synaptic input.. Biol. Cybern. 95, pp. 1-19. DOI: 10.1007/s00422-006-00686.

Buschman, T. J., Denovellis, E. L., Diogo, C., Bullock, D., and Miller, E. K. (2012). Synchronous Oscillatory Neural Ensembles for Rules in the Prefrontal Cortex. Neuron 76, pp. 838-846. DOI: 10.1016/j.neuron. 2012.09.029.

Butcher, J. C. (2003). Numerical Methods for Ordinary Differential Equations. 1st Ed. New York: John Wiley \& Sons.

Buzsáki, G. (1984). Feed-forward inhibition in the hippocampal formation. Prog. Neurobiol. 22, pp. 131-153. DOI: 10.1016/0301-0082(84)90023-6.

Buzsáki, G. (2010). Neural syntax: cell assemblies, synapsembles, and readers.. Neuron 68, pp. 362-85. DOI: $10.1016 / \mathrm{j}$.neuron. 2010.09.023. 
Canio, G. D., Stoyanov, S., Larsen, J. C., Hallam, J., Kovalev, A., Kleinteich, T., Gorb, S. N., and Manoonpong, P. (2016a). A robot leg with compliant tarsus and its neural control for efficient and adaptive locomotion on complex terrains. Art. Life and Robot. 21, pp. 274-281. DOI: 10.1007/s10015-016-0296-3.

Canio, G. D., Stoyanov, S., Balmori, I. T., Larsen, J. C., and Manoonpong, P. (2016b). "Adaptive Combinatorial Neural Control for Robust Locomotion of a Biped Robot". From Animals to Animats 14: Proceedings of the 11th International Conference on Simulation of Adaptive Behavior. Cham: Springer International Publishing, pp. 317-328.

Cheng, Z., Deng, Z., Hu, X., Zhang, B., and Yang, T. (2015). Efficient reinforcement learning of a reservoir network model of parametric working memory achieved with a cluster population winner-take-all readout mechanism. J. Neurophysiol. 114, pp. 3296-3305. DOI: 10.1152/jn.00378. 2015.

Chistiakova, M., Bannon, N. M., Bazhenov, M., and Volgushev, M. (2014). Heterosynaptic Plasticity: Multiple Mechanisms and Multiple Roles. Neuroscientist 20, pp. 483-498. DOI: $10.1177 / 1073858414529829$.

Chklovskii, D. B., Mel, B. W., and Svoboda, K. (2004). Cortical rewiring and information storage. Nature 431, pp. 782-788. DOI: 10.1038/nature03012.

Christophel, T. B., Klink, P. C., Spitzer, B., Roelfsema, P. R., and Haynes, J. D. (2017). The Distributed Nature of Working Memory. Trends Cogn. Sci. 21, pp. 111-124. DOI: 10.1016/j.tics.2016.12.007.

Chua, J. J. E., Kindler, S., Boyken, J., and Jahn, R. (2010). The architecture of an excitatory synapse. J. Cell. Sci. 123, pp. 819-823. DOI: 10.1242/jcs. 052696.

Clarac, F. and Pearlstein, E. (2007). Invertebrate preparations and their contribution to neurobiology in the second half of the 20th century. Brain Res. Rev. 54, pp. 113-161. DOI: $10.1016 / \mathrm{j}$. brainresrev.2006.12.007.

Cohen, N. and Squire, L. R. (1980). Preserved learning and retention of patternanalyzing skill in amnesia: dissociation of knowing how and knowing that. Science 210, pp. 207-210. DOI: 10.1126/science.7414331.

Compte, A., Brunel, N., Goldman-Rakic, P. S., and Wang, X.-J. (2000). Synaptic mechanisms and network dynamics underlying spatial working memory in a cortical network model.. Cereb. Cortex 10, pp. 910-923. DOI: 10.1093/cercor/10.9.910.

Constantinidis, C., Franowicz, M. N., and Goldman-Rakic, P. S. (2001). The sensory nature of mnemonic representation in the primate prefrontal cortex.. Nat. Neurosci. 4, pp. 311-6. DOI: $10.1038 / 85179$.

Constantinidis, C. and Klingberg, T. (2016). The neuroscience of working memory capacity and training. Nat. Rev. Neurosci. 17, pp. 438-449. DOI: 10.1038/nrn. 2016. 43.

Constantinidis, C. and Wang, X.-J. (2004). A Neural Circuit Basis for Spatial Working Memory. Neuroscientist 10, pp. 553-565. DOI: 10.1177/1073858404268742.

Conway, A. R. A., Kane, M. J., Bunting, M. F., Hambrick, D. Z., Wilhelm, O., and Engle, R. W. (2005). Working memory span tasks: A methodological review and user's guide. Psychon. Bull. Rev. 12, pp. 769-786. DOI: 10.3758/BF03196772.

Cooley, J. W. and Tukey, J. W. (1965). An Algorithm for the Machine Calculation of Complex Fourier Series. Math. Comput. 19, p. 297. DOI: 10.2307/2003354.

Courtney, S. N., Ungerleider, L. G., Keil, K., and Haxby, J. V. (1997). Transient and sustained activity in a distributed neural system for human working memory. Nature 386, pp. 608-611. DOI: 10.1038/386608a0.

Cowan, N. (1988). Evolving conceptions of memory storage, selective attention, and their mutual constraints within the human information-processing system.. Psychol. Bull. 104, pp. 163-191. DOI: 10.1037/0033-2909.104.2.163. 
Cowan, N. (2001). The magical number 4 in short-term memory: A reconsideration of mental storage capacity. Behav. Brain Sci. 24, S0140525X01003922. DOI: 10.1017/ S0140525X01003922.

Cowan, N. (2008). What are the differences between long-term, short-term, and working memory? Nelson. Prog. Brain Res. 169, pp. 323-338. DOI: 10.1016/S00796123(07)00020-9. What.

Crespi, A. and Ijspeert, A. J. (2008). Online Optimization of Swimming and Crawling in an Amphibious Snake Robot. IEEE Trans. Robot. 24, pp. 75-87. DOI: 10.1109/ TRO. 2008.915426.

Cruse, H., Bartling, C., Dreifert, M., Schmitz, J., Brunn, D. E., Dean, J., and Kindermann, T. (1995). Walking: A Complex Behavior Controlled by Simple Networks. Adapt. Behav. 3, pp. 385-418. DOI: 10.1177/105971239500300403.

Curtis, C. E. and D'Esposito, M. (2003). Persistent activity in the prefrontal cortex during working memory. Trends Cogn. Sci. 7, pp. 415-423. DOI: 10.1016/S13646613(03) 00197-9.

Dasgupta, S., Wörgötter, F., and Manoonpong, P. (2013). Information dynamics based self-adaptive reservoir for delay temporal memory tasks. Evolv. Sys. 4, pp. 1-15. DOI: $10.1007 / \mathrm{s} 12530-013-9080-\mathrm{y}$.

Dayan, P. and Abbott, L. F. (2001). Theoretical Neuroscience: Computational and Mathematical Modeling of Neural Systems. 1sr. USA: MIT Press.

Deco, G. and Rolls, E. T. (2003). Attention and working memory: A dynamical model of neuronal activity in the prefrontal cortex. Eur. J. Neurosci. 18, pp. 2374-2390. DOI: $10.1046 / j .1460-9568.2003 .02956 . x$.

Delcomyn, F. (1980). Neural basis of rhythmic behavior in animals. Science 210, pp. 492-498. DOI: $10.1126 /$ science. 7423199.

D'Esposito, M. (2007). From cognitive to neural models of working memory. Philos. Trans. R. Soc. Lond., B, Biol. Sci. 362, pp. 761-772. DOI: 10.1098/rstb.2007. 2086.

D'Esposito, M. and Postle, B. R. (2015). The Cognitive Neuroscience of Working Memory. Annu. Rev. Psychol. 66, pp. 115-142. DOI: 10 .1146/annurev-psych010814-015031.

Diamond, A. (2014). Executive Functions. Annu. Rev. Clin. Psychol. 64, pp. 135-168. DOI: 10.1146/annurev-psych-113011-143750. Executive.

Drover, J. D. (2014). Timing over Tuning: Overcoming the Shortcomings of a Line Attractor during a Working Memory Task. PLoS Comput. Biol. 10, e1003437. DOI: 10.1371/journal.pcbi.1003437.

Duffing, G. (1918). Erzwungene Schwingungen bei Veränderlicher Eigenfrequenz. Vol. 1. Braunschweig: F. Vieweg u. Sohn.

Durstewitz, D., Seamans, J. K., and Sejnowski, T. J. (2000). Neurocomputational models of working memory.. Nat. Neurosci. 3 Suppl, pp. 1184-1191. DOI: 10.1038/ 81460.

Eichenbaum, H. B. (2000). A cortical-hippocampal system for declarative memory.. Nat. Rev. Neurosci. 1, pp. 41-50. DOI: 10.1038/35036213.

Eichenbaum, H. B. (2011). The Cognitive Neuroscience of Memory: An Introduction. 2nd. Oxford: Oxford University Press.

Enel, P., Procyk, E., Quilodran, R., and Dominey, P. F. (2016). Reservoir Computing Properties of Neural Dynamics in Prefrontal Cortex. PLoS Comput. Biol. 12, e1004967. DOI: 10.1371/journal.pcbi.1004967.

Eriksson, J., Vogel, E. K., Lansner, A., Bergström, F., and Nyberg, L. (2015). Neurocognitive Architecture of Working Memory. Neuron 88, pp. 33-46. DOI: 10.1016/j . neuron.2015.09.020. 
Fauth, M., Wörgötter, F., and Tetzlaff, C. (2015). The Formation of Multi-synaptic Connections by the Interaction of Synaptic and Structural Plasticity and Their Functional Consequences. PLoS Comput. Biol. 11, e1004031. DOI: 10.1371/journal . pcbi. 1004031.

Fino, E. and Yuste, R. (2011). Dense inhibitory connectivity in neocortex. Neuron 69, pp. 1188-1203. DOI: 10.1016/j . neuron. 2011.02.025.

Fitzhugh, R. (1961). Impulses and Physiological States in Theoretical Models of Nerve Membrane. Biophys. J. 1, pp. 445-466. DOI: 10.1016/S0006-3495(61)86902-6.

Foth, E. and Bässler, U. (1985). Leg movements of stick insects walking with five legs on a treadwheel and with one leg on a motor-driven belt. I. General results and 1:1-coordination.. Biol. Cybern. 51, pp. 313-318. DOI: 10.1007/BF00336918.

Fukai, T. and Tanaka, S. (1997). A Simple Neural Network Exhibiting Selective Activation of Neuronal Ensembles: From Winner-Take-All to Winners-Share-All. Neural Comput. 9, pp. 77-97. DOI: 10.1162/neco.1997.9.1.77.

Funahashi, S., Bruce, C. J., and Goldman-Rakic, P. S. (1989). Mnemonic coding of visual space in the monkey's dorsolateral prefrontal cortex. J. Neurophysiol. 61, pp. 331-349.

Furuta, K. (2003). “Control of Pendulum: From Super Mechano-System to Human Adaptive Mechatronics". Proceedings of the IEEE Conference on Decision and Control. Piscataway, NJ: IEEE, pp. 1498-1507.

Fuster, J. M. and Alexander, G. E. (1971). Neuron activity related to short-term memory. Science 173, pp. 652-654. DOI: 10.1126/science.173.3997.652.

Garagnani, M., Wennekers, T., and Pulvermüller, F. (2009). Recruitment and Consolidation of Cell Assemblies for Words by Way of Hebbian Learning and Competition in a Multi-Layer Neural Network. Cognit. Comput. 1, pp. 160-176. DOI: 10.1007/s12559-009-9011-1.

Gathercole, S. E., Brown, L., and Pickering, S. J. (2003). Working memory assessments at school entry as longitudinal predictors of National Curriculum attainment levels. Educ. Child. Psychol. 20, pp. 109-122.

Genzel, L., Rossato, J. I., Jacobse, J., Grieves, R. M., Spooner, P. A., Battaglia, F. P., Fernández, G., and Morris, R. G. (2017). The Yin and Yang of Memory Consolidation: Hippocampal and Neocortical. PLoS Biol. 15, pp. 1-26. DOI: 10.1371/ journal . pbio. 2000531.

Gerstner, W. and Kistler, W. M. (2002). Mathematical formulations of Hebbian learning.. Biol. Cybern. 87, pp. 404-15. DOI: 10.1007/s00422-002-0353-y.

Gerstner, W., Kistler, W. M., Naud, R., and Paninski, L. (2014). Neuronal Dynamics: From Single Neurons to Networks and Models of Cognition. 1st. Cambridge, UK: Cambridge University Press.

Goldbeter, A., Gérard, C., Gonze, D., Leloup, J. C., and Dupont, G. (2012). Systems biology of cellular rhythms. FEBS Lett. 586, pp. 2955-2965. DOI: 10 . 1016 / j . febslet.2012.07.041.

Goldman-Rakic, P. S. (1995). Cellular basis of working memory. Neuron 14, pp. 477485. DOI: $10.1016 / 0896-6273$ (95) 90304-6.

Graupner, M. and Brunel, N. (2010). Mechanisms of induction and maintenance of spike-timing dependent plasticity in biophysical synapse models. Front. Comput. Neurosci. 4, pp. 1-19. DOI: 10.3389/fncom. 2010.00136.

Gros, C. (2009). Cognitive computation with autonomously active neural networks: An emerging field. Cognit. Comput. 1, pp. 77-90. DOI: 10.1007/s12559-008-90009.

Guertin, P. A. (2009). The mammalian central pattern generator for locomotion. Brain Res. Rev. 62, pp. 45-56. DOI: 10.1016/j . brainresrev. 2009.08.002. 
Guertin, P. A. (2013). Central pattern generator for locomotion: Anatomical, physiological, and pathophysiological considerations. Front. Neurol. 3 FEB, pp. 1-15. DOI: 10.3389/fneur. 2012.00183 .

Harris, K. D. and Mrsic-Flogel, T. D. (2013). Cortical connectivity and sensory coding.. Nature 503, pp. 51-8. DOI: 10.1038/nature12654.

Harris-Warrick, R. M. and Cohen, A. H. (1985). Serotonin modulates the central pattern generator for locomotion in the isolated lamprey spinal cord.. J. Exp. Biol. 116, pp. $27-46$.

Haykin, S. S. (2002). Adaptive Filter Theory. 5th ed. Upper Saddle River, NJ: Prentice Hall.

Hebb, D. O. (1949). The Organization of Behavior. 1st ed. New York: Wiley.

Hellgren, J. and Grillner, S. (1992). Computer simulation of the segmental neural network generating locomotion in lamprey by using populations of network interneurons. Biol. Cybern. 68, pp. 1-13. DOI: 10.1007/BF00203132.

Henson, R. N. (1998). Short-term memory for serial order. Cogn. Psychol. 36, pp. 73137. DOI: $10.1006 / \operatorname{cogp} .1998 .0685$.

Herculano-Houzel, S. (2009). The human brain in numbers: a linearly scaled-up primate brain. Front. Hum. Neurosci. 3, pp. 1-11. DOI: 10.3389/neuro. 09.031. 2009.

Hindmarsh, A. C. (1983). "ODEPACK, A Systematized Collection of ODE Solvers". IMACS Transactions on Scientific Computation: Scientific Computing. Ed. by R. S. Stepleman, C. Carver, R. Peskin, W. F. Ames, and R. Vienevetsky. Amsterdam: North-Holland, pp. 55-63.

Hodgkin, A. L. and Huxley, A. F. (1952). A quantitative description of membrane current and its application to conduction and excitation in nerve. J. Physiol. (Lond.) 117, pp. 500-544. DOI: 10.1113/jphysiol .1952 . sp004764.

Holt, K. G., Hamill, J., and Andres, R. O. (1990). The force-driven harmonic oscillator as a model for human locomotion. Hum. Mov. Sci. 9, pp. 55-68. DOI: 10.1016/01679457 (90) $90035-\mathrm{C}$.

Holtmaat, A. and Caroni, P. (2016). Functional and structural underpinnings of neuronal assembly formation in learning. Nat. Neurosci. 19, pp. 1553-1562. DOI: $10.1038 / \mathrm{nn} .4418$.

Hooper, S. L. (2001). "Central Pattern Generators". Encyclopedia of Life Sciences. New York: John Wiley \& Sons, Ltd.

Hopfield, J. J. (1982). Neural networks and physical systems with emergent collective computational abilities. Proc. Natl. Acad. Sci. USA 79, pp. 2554-2558. DOI: 10.1073/ pnas.79.8.2554.

Hopfield, J. J. (1984). Neurons with graded response have collective computational properties like those of two-state neurons.. Proc. Natl. Acad. Sci. USA 81, pp. 30883092. DOI: $10.1073 /$ pnas.81.10.3088.

Hulme, C., Maughan, S., and Brown, G. D. A. (1991). Memory for familiar and unfamiliar words: Evidence for a long-term memory contribution to short-term memory span.. J. Mem. Lang. 30, pp. 685-701. DOI: 10 .1016/0749-596X (91) 90032F.

Hunsaker, M. R. and Kesner, R. P. (2013). The operation of pattern separation and pattern completion processes associated with different attributes or domains of memory. Neurosci. Biobehav. Rev. 37, pp. 36-58. DOI: 10.1016/j . neubiorev . 2012. 09.014.

Hurlstone, M. J., Hitch, G. J., and Baddeley, A. D. (2014). Memory for serial order across domains: An overview of the literature and directions for future research.. Psychol. Bull. 140, pp. 339-373. DOI: 10.1037/a0034221. 
Hussar, C. R. and Pasternak, T. (2012). Memory-guided sensory comparisons in the prefrontal cortex: contribution of putative pyramidal cells and interneurons.. J. Neurosci. 32, pp. 2747-2761. DOI: 10.1523/JNEUROSCI . 5135-11. 2012.

Ijspeert, A. J. (2008). Central pattern generators for locomotion control in animals and robots: a review.. Neural Netw. 21, pp. 642-653. DOI: 10.1016/j .neunet. 2008. 03. 014.

Inoue, K., Sumi, T., and Ma, S. (2007). "CPG-based control of a simulated snake-like robot adaptable to changing ground friction". Proceedings of the 2007 IEEE/RSJ International Conference on Intelligent Robots and Systems. San Diego: IEEE, pp. 19571962.

Isaacson, J. S. and Scanziani, M. (2011). How inhibition shapes cortical activity.. Neuron 72, pp. 231-43. DOI: $10.1016 / \mathrm{j}$. neuron. 2011.09. 027.

Jaeger, H. (2001). The "echo state" approach to analysing and training recurrent neural networks. Tech. rep. GMD - German National Research Institute for Computer Science.

Jaeger, H. (2002). Short term memory in echo state networks. Tech. rep. GMD - German National Research Institute for Computer Science.

Jaeger, H. and Eck, D. (2008). "Can't Get You Out of My Head: A Connectionist Model of Cyclic Rehearsal". Modeling Communication with Robots and Virtual Humans: Second ZiF Research Group International Workshop on Embodied Communication in Humans and Machines, Bielefeld, Germany, April 5-8, 2006, Revised Selected Papers. Ed. by I. Wachsmutch and G. Knoblich. Berlin, Heidelberg: Springer Berlin Heidelberg, pp. 310-335.

Jaeggi, S. M., Buschkuehl, M., Jonides, J., and Perrig, W. J. (2008). Improving fluid intelligence with training on working memory.. Proc. Natl. Acad. Sci. USA 105, pp. 6829-6833. DOI: 10.1073/pnas.0801268105.

Jaeggi, S. M., Buschkuehl, M., Perrig, W. J., and Meier, B. (2010). The concurrent validity of the N-back task as a working memory measure. Memory 18, pp. 394412. DOI: $10.1080 / 09658211003702171$.

Jaeggi, S. M., Seewer, R., Nirkko, A. C., Eckstein, D., Schroth, G., Groner, R., and Gutbrod, K. (2003). Does excessive memory load attenuate activation in the prefrontal cortex? Load-dependent processing in single and dual tasks: Functional magnetic resonance imaging study. Neuroimage 19, pp. 210-225. DOI: 10.1016/S10538119 (03) 00098-3.

James, W. (1890). The Principles of Psychology. 1st ed. New York: Holt.

Jeneson, A. and Squire, L. R. (2011). Working memory, long-term memory, and medial temporal lobe function. Learn. Mem. 19, pp. 15-25. DOI: 10.1101/lm.024018.111.

Johannsen, L., Li, K. Z. H., Chechlacz, M., Bibi, A., Kourtzi, Z., and Wing, A. M. (2013). Functional neuroimaging of the interference between working memory and the control of periodic ankle movement timing. Neuropsychologia 51, pp. 2142-2153. DOI: $10.1016 / \mathrm{j}$.neuropsychologia.2013.07.009.

Jones, E., Oliphant, T., Peterson, P., and Al, E. (2001). SciPy: Open source scientific tools for Python.

Jun, J. K., Miller, P., Hernandez, A., Zainos, A., Lemus, L., Brody, C. D., and Romo, R. (2010). Heterogenous Population Coding of a Short-Term Memory and Decision Task. J. Neurosci. 30, pp. 916-929. DOI: 10.1523/JNEUROSCI . 2062-09. 2010.

Juola, J. F., Fischler, I., Wood, C. T., and Atkinson, R. C. (1971). Recognition Time for Information Stored in Long-Term Memory. Percept. Psychophys. 10, pp. 8-14. DOI: 10.3758/BF03205757. 
Kamiński, J. (2017). Intermediate-Term Memory as a Bridge between Working and Long-Term Memory. J. Neurosci. 37, pp. 5045-5047. DOI: 10 . 1523 / JNEUROSCI . 0604-17. 2017.

Kamiński, J., Sullivan, S., Chung, J. M., Ross, I. B., Mamelak, A. N., and Rutishauser, U. (2017). Persistently active neurons in human medial frontal and medial temporal lobe support working memory. Nat. Neurosci. 20, pp. 590-601. DOI: 10.1038/nn. 4509.

Kandel, E. R., Schwartz, J. H., and Jessel, T. M. (2000). Principles of Neural Science. 4/e. United States of America: McGraw-Hill Companies.

Kane, M. J., Brown, L. H., McVay, J. C., Silvia, P. J., Myin-Germeys, I., and Kwapil, T. R. (2007a). For whom the mind wanders, and when: An experience-sampling study of working memory and executive control in daily life. Psychol. Sci. 18, pp. 614-621. DOI: $10.1111 / j .1467-9280.2007 .01948 . x$.

Kane, M. J., Conway, A. R. A., Miura, T. K., and Colflesh, G. J. H. (2007b). Working memory, attention control, and the n-back task: A question of construct validity.. J. Exp. Psychol. Learn. Mem. Cogn. 33, pp. 615-622. DOI: 10.1037/0278-7393.33.3. 615.

Katkov, M., Romani, S., and Tsodyks, M. (2017). Memory Retrieval from First Principles. Neuron 94, pp. 1027-1032. DOI: 10.1016/j . neuron . 2017.03.048.

Kimura, H., Fukuoka, Y., and Konaga, K. (2001). Adaptive dynamic walking of a quadruped robot using a neural system model. Adv. Robot. 15, pp. 859-878. DOI: 10.1163/156855301317198179.

Kirchner, W. K. (1958). Age differences in short-term retention of rapidly changing information.. J. Exp. Psychol. 55, pp. 352-358. DOI: 10.1037/h0043688.

Kohonen, T. (1982). Self-organized formation of topologically correct feature maps. Biol. Cybern. 43, pp. 59-69. DOI: 10.1007/BF00337288.

Kohring, G. A. (1990). Convergence time and finite size effects in neural networks. J. Phys. A Math. Gen. 23, pp. 2237-2241. DOI: 10.1088/0305-4470/23/11/047.

Koppe, G., Gruppe, H., Sammer, G., Gallhofer, B., Kirsch, P., and Lis, S. (2014). Temporal unpredictability of a stimulus sequence affects brain activation differently depending on cognitive task demands. Neuroimage 101, pp. 236-244. DOI: 10.1016/j. neuroimage. 2014.07.008.

Kubota, K. and Niki, H. (1971). Prefrontal cortical unit activity and delayed alternation performance in monkeys.. J. Neurophysiol. 34, pp. 337-347.

Kubota, Y., Karube, F., Nomura, M., and Kawaguchi, Y. (2016). The Diversity of Cortical Inhibitory Synapses. Front. Neural Circuits 10, pp. 1-15. DOI: 10.3389/ fncir.2016.00027.

Kullmann, D. M., Moreau, A. W., Bakiri, Y., and Nicholson, E. (2012). Plasticity of Inhibition. Neuron 75, pp. 951-962. DOI: 10.1016/j .neuron. 2012.07.030.

Kuznetsov, Y. A. (1998). Elements of applied bifurcation theory. 2nd Ed. New York: Springer.

Kyllonen, P. C. and Christal, R. E. (1990). Reasoning ability is (little more than) working-memory capacity?!. Intelligence 14 , pp. 389-433. DOI: 10 .1016/S01602896 (05) 80012-1.

Laje, R. and Buonomano, D. V. (2013). Robust timing and motor patterns by taming chaos in recurrent neural networks.. Nat. Neurosci. 16, pp. 925-933. DOI: 10.1038/ $\mathrm{nn} .3405$.

Landsman, A. and Slotine, J.-J. (2012). Control of traveling-wave oscillations and bifurcation behavior in central pattern generators. Phys. Rev. E 86, pp. 1-5. DOI: 10.1103/PhysRevE. 86.041914. 
Lansner, A. (2009). Associative memory models: from the cell-assembly theory to biophysically detailed cortex simulations.. Trends Neurosci. 32, pp. 178-86. DOI: $10.1016 / j$.tins . 2008.12.002.

Large, A. M., Vogler, N. W., Mielo, S., and Oswald, A.-M. M. (2016). Balanced feedforward inhibition and dominant recurrent inhibition in olfactory cortex. Proc. Natl. Acad. Sci. USA 113, pp. 2276-2281. DOI: 10.1073/pnas. 1519295113.

Laughlin, S. B. (2001). Energy as a constraint on the coding and processing of sensory information. Curr. Opin. Neurobiol. 11, pp. 475-480. DOI: 10.1016/S0959-4388(00) 00237-3.

Legenstein, R. and Maass, W. (2007). Edge of chaos and prediction of computational performance for neural circuit models.. Neural Netw. 20, pp. 323-34. DOI: 10.1016/ j.neunet.2007.04.017.

Leone, C., Feys, P., Moumdjian, L., D’Amico, E., Zappia, M., and Patti, F. (2017). Cognitive-motor dual-task interference: A systematic review of neural correlates. Neurosci. Biobehav. Rev. 75, pp. 348-360. DOI: 10.1016/j . neubiorev.2017.01.010.

Litwin-Kumar, A. and Doiron, B. (2014). Formation and maintenance of neuronal assemblies through synaptic plasticity. Nat. Commun. 5, p. 5319. DOI: 10.1038/ ncomms 6319 .

Liu, J. K. (2011). Learning Rule of Homeostatic Synaptic Scaling: Presynaptic Dependent or Not. Neural Comput. 23, pp. 3145-3161. DOI: 10.1162/NECO\_a \_00210.

Lu, Z., Ma, S., Li, B., and Wang, Y. (2005). "Serpentine Locomotion of a Snakelike Robot Controlled by Cyclic Inhibitory CPG Model". Proceedings of the 2005 IEEE/RSJ International Conference on Intelligent Robots and Systems. Edmonton: IEEE, pp. 96-101.

Lukoševičius, M. and Jaeger, H. (2009). Reservoir computing approaches to recurrent neural network training. Comp. Sci. Rev. 3, pp. 127-149. DOI: doi : $10.1016 /$ j . cosrev.2009.03.005.

Lukoševičius, M., Jaeger, H., and Schrauwen, B. (2012). Reservoir computing trends. Künstl. Intell. 26, pp. 365-371. DOI: 10.1007/s13218-012-0204-5.

Lüscher, C. and Malenka, R. C. (2012). NMDA receptor-dependent long-term potentiation and long-term depression (LTP/LTD). Cold Spring Harb Perspect Biol 4, pp. 1-15. DOI: 10.1101/cshperspect . a005710.

Maass, W. (2011). "Liquid State Machines: Motivation, Theory, and Applications". Computability in Context. Ed. by S. B. Cooper and A. Sorbi. London: Imperial College Press, pp. 275-296.

Maass, W., Joshi, P., and Sontag, E. D. (2007). Computational aspects of feedback in neural circuits. PLoS Comput. Biol. 3, e165. DOI: 10.1371/journal .pcbi. 0020165.

Maass, W., Natschläger, T., and Markram, H. (2002). Real-time computing without stable states: a new framework for neural computation based on perturbations.. Neural Comput. 14, pp. 2531-2560. DOI: 10.1162/089976602760407955.

Maass, W. and Sontag, E. D. (1999). Analog neural nets with Gaussian or other common noise distributions cannot recognize arbitrary regular languages. Neural Comput. 11, pp. 771-782. DOI: 10.1162/089976699300016656.

Malenka, R. C. and Bear, M. F. (2004). LTP and LTD: An embarrassment of riches. Neuron 44, pp. 5-21. DOI: 10.1016/j.neuron.2004.09.012.

Mante, V., Sussillo, D., Shenoy, K. V., and Newsome, W. T. (2013). Context-dependent computation by recurrent dynamics in prefrontal cortex. Nature 503, pp. 78-84. DOI: $10.1038 /$ nature 12742 .

Marder, E. and Bucher, D. (2001). Central pattern generators and the control of rhythmic movements.. Curr. Biol. 11, R986-996. DOI: 10.1016/S0960-9822 (01) 00581-4. 
Markram, H., Wang, Y., and Tsodyks, M. (1998). Differential signaling via the same axon of neocortical pyramidal neurons. Proc. Natl. Acad. Sci. USA 95, pp. 5323-8. DOI: $10.1073 /$ pnas .95.9.5323.

Martin, S. J., Grimwood, P. D., and Morris, R. G. M. (2000). Synaptic Plasticity and Memory: An Evaluation of the Hypothesis. Annu. Rev. Neurosci. 23, pp. 649-711. DOI: 10.1146/annurev . neuro.23.1.649.

Marton, K. and Eichorn, N. (2014). Interaction Between Working Memory and LongTerm Memory. Z. Psychol. 222, pp. 90-99. DOI: 10.1027/2151-2604/a000170.

Matsuoka, K. (1985). Sustained oscillations generated by mutually inhibiting neurons with adaptation. Biol. Cybern. 52, pp. 367-376. DOI: 10.1007/BF00449593.

Mayford, M., Siegelbaum, S. A., Kandel, E. R., Long-term, N. R.-d., Lüscher, C., Malenka, R. C., Harris, K. M., Weinberg, R. J., Smart, T. G., Paoletti, P., and Südhof, C. (2012). Synapses and Memory Storage. Cold Spring Harb Perspect Biol 4, a005751. DOI: $10.1101 /$ cshperspect.a005751.

Mazor, O. and Laurent, G. (2005). Transient dynamics versus fixed points in odor representations by locust antennal lobe projection neurons. Neuron 48, pp. 661-673. DOI: $10.1016 / j$.neuron. 2005.09.032.

McCulloch, W. S. and Pitts, W. (1943). A logical calculus of the ideas immanent in nervous activity. Bull Math Biophys 5, pp. 115-133. DOI: 10.1007/BF02478259.

Mi, Y., Katkov, M., and Tsodyks, M. (2017). Synaptic Correlates of Working Memory Capacity. Neuron 93, pp.323-330. DOI: 10.1016/j.neuron.2016.12.004.

Miller, G. A. (1956). The magical number seven, plus or minus two: some limits on our capacity for processing information.. Psychol. Rev. 63, pp. 81-97. DOI: 10.1037/h0043158.

Miller, S. and Scott, P. (1977). The spinal locomotor generator. Exp. Brain Res. 30, pp. 387-403. DOI: $10.1007 /$ BF00237264.

Mongillo, G., Barak, O., and Tsodyks, M. (2008). Synaptic theory of working memory.. Science 319, pp. 1543-1546. DOI: 10.1126/science.1150769.

Muir, D. R. and Cook, M. (2014). Anatomical constraints on lateral competition in columnar cortical architectures.. Neural Comput. 26, pp. 1624-66. DOI: 10.1162/ NECO\_a $\backslash_{-} 00613$.

Murray, J. D., Bernacchia, A., Roy, N. A., Constantinidis, C., Romo, R., and Wang, X.-J. (2017). Stable population coding for working memory coexists with heterogeneous neural dynamics in prefrontal cortex. Proc. Natl. Acad. Sci. USA 114, pp. 394-399. DOI: $10.1073 /$ pnas. 1619449114.

Nachstedt, T. and Tetzlaff, C. (2017). Working Memory Requires a Combination of Transient and Attractor-Dominated Dynamics to Process Unreliably Timed Inputs. Sci. Rep. 7, p. 2473. DOI: 10.1038/s41598-017-02471-z.

Nachstedt, T., Tetzlaff, C., and Manoonpong, P. (2017). Fast dynamical coupling enhances frequency adaptation of oscillators for robotic locomotion control. Front. Neurorobot. 11, pp.1-14. DOI: 10.3389/fnbot.2017.00014.

Nachstedt, T., Wörgötter, F., and Manoonpong, P. (2012). “Adaptive neural oscillator with synaptic plasticity enabling fast resonance tuning". Proceedings of the 2012 International Conference on Artificial Neural Networks. Lausanne: Springer, pp. 451458.

Nachstedt, T., Wörgötter, F., Manoonpong, P., Ariizumi, R., Ambe, Y., and Matsuno, F. (2013). "Adaptive neural oscillators with synaptic plasticity for locomotion control of a snake-like robot with screw-drive mechanism". IEEE International Conference on Robotics and Automation. Piscataway, NJ: IEEE, pp. 3389-3395.

Nadel, L. and Hardt, O. (2011). Update on memory systems and processes.. Neuropsychopharmacology 36, pp. 251-73. DOI: 10.1038/npp. 2010.169. 
Nadim, F. and Manor, Y. (2000). The role of short-term synaptic dynamics in motor control. Curr. Opin. Neurobiol. 10, pp. 683-690. DOI: 10.1016/S0959-4388(00) 00159-8.

Nakamura, Y., Mori, T., Sato, M.-a., and Ishii, S. (2007). Reinforcement learning for a biped robot based on a CPG-actor-critic method. Neural Netw. 20, pp. 723-735. DOI: $10.1016 / \mathrm{j}$. neunet.2007.01.002.

Nassour, J., Hénaff, P., Benouezdou, F., and Cheng, G. (2014). Multi-layered multipattern CPG for adaptive locomotion of humanoid robots. Biol. Cybern. 108, pp. 291-303. DOI: 10.1007/s00422-014-0592-8.

Nicoll, R. A. and Roche, K. W. (2013). Long-term potentiation: Peeling the onion. Neuropharmacology 74, pp. 18-22. DOI: 10.1016/j . neuropharm. 2013.02.010.

Owen, A. M., McMillan, K. M., Laird, A. R., and Bullmore, E. (2005). N-back working memory paradigm: A meta-analysis of normative functional neuroimaging studies. Hum. Brain Mapp. 25, pp. 46-59. DOI: 10.1002/hbm. 20131.

Palm, G., Knoblauch, A., Hauser, F., and Schüz, A. (2014). Cell assemblies in the cerebral cortex. Biol. Cybern. 108, pp. 559-572. DOI: 10.1007/s00422-014-0596-4.

Pascanu, R. and Jaeger, H. (2011). A neurodynamical model for working memory.. Neural Netw. 24, pp. 199-207. DOI: 10.1016/j.neunet.2010.10.003.

Pasemann, F., Hild, M., and Zahedi, K. (2003). "SO(2)-Networks as Neural Oscillators". Computational methods in Neural Modeling. Ed. by J. Mira. Berlin / Heidelberg: Springer, pp. 1042-1042.

Pearson, K. G. and Duysens, J. (1976). "Function of Segmental Reflexes in the Control of Stepping in Cockroaches and Cats". Neural Control in Locomotion. Ed. by R. E. Herman, S. Grillner, D. Stuart, and P. Stein. New York: Plenum Press, pp. 519-537.

Pereda, A. E. (2014). Electrical synapses and their functional interactions with chemical synapses. Nat. Rev. Neurosci. 15, pp. 250-263. DOI: 10.1038/nrn3708.

Peterson, L. R. and Peterson, M. J. (1959). Short-term retention of individual verbal items.. J. Exp. Psychol. Gen. 58, pp. 193-8. DOI: 10.1037/h0049234.

Pfeifer, R., Lungarella, M., and Iida, F. (2007). Self-organization, embodiment, and biologically inspired robotics.. Science 318, pp. 1088-93. DOI: 10.1126/science. 1145803.

Pinto, C. M. A., Rocha, D., and Santos, C. P. (2012). Hexapod robots: new CPG model for generation of trajectories. SIAM J. Numer. Anal. 7, pp. 15-26. DOI: 10.1063/1.3636775.

Poirier, M., Dhir, P., Saint-Aubin, J., Tehan, G., and Hampton, J. (2011). “The Influence of Semantic Memory on Verbal Short-term Memory". European Perspectives on Cognitive Science. Sofia: New Bulgarian University Press.

Potter, S. M., El Hady, A., and Fetz, E. E. (2014). Closed-loop neuroscience and neuroengineering. Front. Neural Circuits 8, pp. 2013-2015. DOI: 10.3389/fncir. 2014.00115.

Pribram, K. H., Mishkin, M., Rosvold, H. E., and Kaplan, S. J. (1952). Effects on delayed-response performance of lesions of dorsolateral and ventromedial frontal cortex of baboons.. J. Comp. Physiol. Psychol. 45, pp. 565-575. DOI: 10 . 1037 / h0061240.

Rabinovich, M. I., Huerta, R., and Laurent, G. (2008a). Transient Dynamics for Neural Processing. Science 321, pp. 48-50. DOI: 10.1126/science.1155564.

Rabinovich, M. I., Huerta, R., Varona, P., and Afraimovich, V. S. (2008b). Transient cognitive dynamics, metastability, and decision making. PLoS Comput. Biol. 4, pp. 25-30. DOI: 10.1371/journal.pcbi. 1000072. 
Rabinovich, M. I., Varona, P., Selverston, A. I., and Abarbanel, H. D. I. (2006). Dynamical principles in neuroscience. Rev. Mod. Phys. 78, pp. 1213-1265. DOI: 10.1103/ RevModPhys .78.1213.

Rainer, G. and Miller, E. K. (2002). Timecourse of object-related neural activity in the primate prefrontal cortex during a short-term memory task.. Eur. J. Neurosci. 15, pp. 1244-1254. DOI: 10.1046/j.1460-9568.2002.01958.x.

Ranganath, C., Cohen, M. X., and Brozinsky, C. J. (2005). Working memory maintenance contributes to long-term memory formation: neural and behavioral evidence.. J Cogn Neurosci 17, pp. 994-1010. DOI: 10.1162/0898929054475118.

Rayleigh, J. W. S. (1877). The theory of sound. Vol. 1. London: Macmillan and co.

Ren, Q. and Zhao, J. (2007). Adaptive coupling and enhanced synchronization in coupled phase oscillators. Phys. Rev. E 76, pp. 1-6. DOI: 10.1103/PhysRevE. 76. 016207.

Revlin, R. (2013). "Short-Term Memory and Working Memory". Cognition: Theory and Practice. Ed. by K. Feyen. New York: Worth Publishers, pp. 118-149.

Righetti, L., Buchli, J., and Ijspeert, A. J. (2006). Dynamic Hebbian learning in adaptive frequency oscillators. Physica D 216, pp. 269-281. DOI: 10.1016/j . physd. 2006. 02.009.

Righetti, L., Buchli, J., and Ijspeert, A. J. (2009). Adaptive Frequency Oscillators and Applications. Open Cybern. Syst. J. 3, pp. 64-69. DOI: 10 . 2174 / $1874110 \times 00903020064$.

Righetti, L. and Ijspeert, A. J. (2008). "Pattern generators with sensory feedback for the control of quadruped locomotion". Proceedings of the IEEE International Conference on Robotics and Automation. Piscataway, NJ: IEEE, pp. 819-824.

Riley, M. R. and Constantinidis, C. (2016). Role of Prefrontal Persistent Activity in Working Memory. Front. Syst. Neurosci. 9, p. 181. DOI: 10 . 3389 /fnsys . 2015. 00181.

Rogerson, T., Cai, D. J., Frank, A., Sano, Y., Shobe, J., Lopez-Aranda, M. F., and Silva, A. J. (2014). Synaptic tagging during memory allocation.. Nat. Rev. Neurosci. 15, pp. 157-69. DOI: $10.1038 / \mathrm{nrn} 3667$.

Romani, S., Pinkoviezky, I., Rubin, A., and Tsodyks, M. (2013). Scaling laws of associative memory retrieval. Neural Comput. 2544, pp. 2523-2544. DOI: 10.1162/NECO.

Ronsse, R., Lenzi, T., Vitiello, N., Koopman, B., Van Asseldonk, E., De Rossi, S. M. M., Van Den Kieboom, J., Van Der Kooij, H., Carrozza, M. C., and Ijspeert, A. J. (2011). Oscillator-based assistance of cyclical movements: Model-based and model-free approaches. Med. Biol. Eng. Comput. 49, pp. 1173-1185. DOI: 10.1007/s11517-0110816-1.

Rose, N. S., LaRocque, J. J., Riggall, A. C., Gosseries, O., Starrett, M. J., Meyering, E. E., and Postle, B. R. (2016a). Reactivation of latent working memories with transcranial magnetic stimulation. Science 354, pp. 1136-1139. DOI: 10.1126 / science. aah7011.

Rose, N. S., LaRocque, J. J., Riggall, A. C., Gosseries, O., Starrett, M. J., Meyering, E. E., and Postle, B. R. (2016b). Reactivation of latent working memories with transcranial magnetic stimulation. Science 354, pp. 1136-1139. DOI: $10.1126 /$ science . aah7011.

Ryle, G. (1949). The Concept of Mind. 2002 editi. Chicago: University of Chicago Press.

Salazar, R. F., Dotson, N. M., Bressler, S. L., and Gray, C. M. (2012). Content-specific fronto-parietal synchronization during visual working memory. Science 338, pp. 1097-1100. DOI: $10.1126 /$ science. 1224000. 
Sandberg, A., Tegnér, J., and Lansner, A. (2003). A working memory model based on fast Hebbian learning. Network 14, pp. 789-802. DOI: 10.1088/0954-898X \_14\ _4\_309.

Santiago, R. A. (2004). “Context Discerning Multifunction Networks: Reformulating Fixed Weight Neural Networks". Proceedings of the International Joint Conference on Neural Networks. Budapest: IEEE, pp. 189-194.

Santos, C. P., Alves, N., and Moreno, J. C. (2017). Biped Locomotion Control through a Biomimetic CPG-based Controller. J. Intell. Robot. Syst. 85, pp. 47-70. DOI: 10 . 1007/s10846-016-0407-3.

Schrauwen, B., Büsing, L., and Legenstein, R. (2009). “On Computational Power and the Order-Chaos Phase Transition in Reservoir Computing". Advances in Neural Information Processing Systems 21. Red Hook, NY: Curran Associates, Inc., pp. $1425-1432$.

Shatz, C. J. (1992). The Developing Brain. Sci. Am. 267, pp. 60-67. DOI: 10 . $1038 /$ scientificamerican0992-60.

Shriki, O., Hansel, D., and Sompolinsky, H. (2003). Rate models for conductancebased cortical neuronal networks.. Neural Comput. 15, pp. 1809-41. DOI: 10.1162/ 08997660360675053.

Siegel, M., Warden, M. R., and Miller, E. K. (2009). Phase-dependent neuronal coding of objects in short-term memory. Proc. Natl. Acad. Sci. USA 106, pp. 21341-21346. DOI: $10.1073 /$ pnas . 0908193106.

Silvanto, J. (2017). Working Memory Maintenance: Sustained Firing or Synaptic Mechanisms?. Trends Cogn. Sci. 21, pp. 152-154. DOI: 10.1016/j.tics . 2017.01. 009.

Sjöström, P. J., Turrigiano, G. G., and Nelson, S. B. (2001). Rate, Timing, and Cooperativity Jointly Determine Cortical Synaptic Plasticity. Neuron 32, pp. 1149-1164. DOI: 10.1016/S0896-6273(01)00542-6.

Sompolinsky, H., Crisanti, A., and Sommers, H. J. (1988). Chaos in Random Neural Networks. Phys. Rev. Lett. 61, pp. 259-262. DOI: 10.1103/PhysRevLett.61.259.

Spong, M. W. (1995). Swing up control problem for the acrobot. IEEE Control Syst. Mag. N. Y. 15, pp. 49-55. DOI: 10.1109/37.341864.

Squire, L. R. (2004). Memory systems of the brain: A brief history and current perspective. Neurobiol. Learn. Mem. 82, pp. 171-177. DOI: 10.1016/j.nlm.2004.06.005.

Squire, L. R. (2009). The Legacy of Patient H.M. for Neuroscience. Neuron 61, pp. 6-9. DOI: $10.1016 / \mathrm{j}$. neuron. 2008.12.023.

Sreenivasan, K. K., Curtis, C. E., and D'Esposito, M. (2014). Revisiting the role of persistent neural activity during working memory. Trends Cogn. Sci. 18, pp. 82-89. DOI: $10.1016 / j$.tics.2013.12.001.

Steingrube, S., Timme, M., Wörgötter, F., and Manoonpong, P. (2010). Self-organized adaptation of a simple neural circuit enables complex robot behaviour. Nat. Phys. 6, pp. 224-230. DOI: 10.1038/nphys1508.

Stevens, J.-L. R., Law, J. S., Antolík, J., and Bednar, J. a. (2013). Mechanisms for stable, robust, and adaptive development of orientation maps in the primary visual cortex.. J. Neurosci. 33, pp. 15747-66. DOI: 10.1523/JNEUROSCI . 1037-13. 2013.

Stokes, M. G. (2015). 'Activity-silent' working memory in prefrontal cortex: A dynamic coding framework. Trends Cogn. Sci. 19, pp. 394-405. DOI: 10.1016/j .tics. 2015. 05.004.

Stokes, M. G., Kusunoki, M., Sigala, N., Nili, H., Gaffan, D., and Duncan, J. (2013). Dynamic coding for cognitive control in prefrontal cortex. Neuron 78, pp. 364-375. DOI: $10.1016 / j$.neuron. 2013.01.039. 
Strata, P. and Harvey, R. (1999). Dale's principle. Brain Res. Bull. 50, pp. 349-350. DOI: 10.1016/S0361-9230 (99)00100-8.

Stuart, G., Spruston, N., Sakmann, B., and Hausser, M. (1997). Action potential initiation and back propagation in neurons of the mammalian central nervous system. Trends Neurosci. 20, pp. 125-131. DOI: 10.1016/S0166-2236 (96) 10075-8.

Sugase-Miyamoto, Y., Liu, Z., Wiener, M. C., Optican, L. M., and Richmond, B. J. (2008). Short-term memory trace in rapidly adapting synapses of inferior temporal cortex. PLoS Comput. Biol. 4, e1000073. DOI: 10.1371/journal.pcbi. 1000073.

Sullivan, T. J. and Sa, V. R. de (2006). Homeostatic synaptic scaling in self-organizing maps.. Neural Netw. 19, pp. 734-43. DOI: 10.1016/j. neunet.2006.05.006.

Sussillo, D. and Abbott, L. F. (2009). Generating coherent patterns of activity from chaotic neural networks.. Neuron 63, pp. 544-557. DOI: 10.1016/j . neuron. 2009. 07.018.

Sussillo, D. and Abbott, L. F. (2012). Transferring learning from external to internal weights in Echo-State networks with sparse connectivity. PLoS One 7, e37372. DOI: 10.1371/journal. pone.0037372.

Sussillo, D. and Barak, O. (2013). Opening the black box: low-dimensional dynamics in high-dimensional recurrent neural networks.. Neural Comput. 25, pp. 626-49. DOI: $10.1162 / \mathrm{NECO} \backslash \mathrm{a} \backslash$-00409.

Sussillo, D., Churchland, M. M., Kaufman, M. T., and Shenoy, K. V. (2015). A neural network that finds a naturalistic solution for the production of muscle activity. Nat. Neurosci. 18, pp. 1025-1033. DOI: 10.1038/nn. 4042.

Takeuchi, T., Duszkiewicz, A. J., and Morris, R. G. M. (2013). The synaptic plasticity and memory hypothesis: encoding, storage and persistence. Philos. Trans. R. Soc. Lond., B, Biol. Sci. 369, pp. 20130288-20130288. DOI: 10.1098/rstb.2013.0288.

Tao, T., Vu, V., and Krishnapur, M. (2010). Random matrices: Universality of ESDs and the circular law. Ann. Probab. 38, pp. 2023-2065. DOI: 10.1214/10-A0P534.

Tetzlaff, C., Dasgupta, S., Kulvicius, T., and Wörgötter, F. (2015). The Use of Hebbian Cell Assemblies for Nonlinear Computation.. Sci. Rep. 5, p. 12866. DOI: 10.1038/ srep12866.

Tetzlaff, C., Kolodziejski, C., Markelic, I., and Wörgötter, F. (2012a). Time scales of memory, learning, and plasticity. Biol. Cybern. 106, pp. 715-726. DOI: 10.1007/ s00422-012-0529-z.

Tetzlaff, C., Kolodziejski, C., Timme, M., Tsodyks, M., and Wörgötter, F. (2013). Synaptic scaling enables dynamically distinct short- and long-term memory formation. PLoS Comput. Biol. 9, e1003307. DOI: 10.1186/1471-2202-14-S1-P415.

Tetzlaff, C., Kolodziejski, C., Timme, M., and Wörgötter, F. (2011). Synaptic scaling in combination with many generic plasticity mechanisms stabilizes circuit connectivity.. Front. Comput. Neurosci. 5, p. 47. DOI: 10.3389/fncom. 2011.00047.

Tetzlaff, C., Kolodziejski, C., Timme, M., and Wörgötter, F. (2012b). Analysis of Synaptic Scaling in Combination with Hebbian Plasticity in Several Simple Networks. Front. Comput. Neurosci. 6, pp. 1-17. DOI: 10.3389/fncom. 2012.00036.

Tråvén, H. G. C., Brodin, L., Lansner, A., Ekeberg, Ö., Wallén, P., and Grillner, S. (1993). Computer simulations of NMDA and non-NMDA receptor-mediated synaptic drive: sensory and supraspinal modulation of neurons and small networks. $J$. Neurophysiol. 70, pp. 695-709.

Tropea, P., Vitiello, N., Martelli, D., Aprigliano, F., Micera, S., and Monaco, V. (2015). Detecting Slipping-Like Perturbations by Using Adaptive Oscillators. Ann. Biomed. Eng. 43, pp. 416-426. DOI: 10.1007/s10439-014-1175-5. 
Trübutschek, D., Marti, S., Ojeda, A., King, J.-R., Mi, Y., Tsodyks, M., and Dehaene, S. (2017). A theory of working memory without consciousness or sustained activity. Elife 6, e23871. DOI: 10.7554/eLife.23871.

Tsodyks, M. and Feigel'man, M. V. (1988). The Enhanced Storage Capacity in Neural Networks with Low Activity Level. Europhys. Lett. 6, p. 101. DOI: 10.1209/02955075/6/2/002.

Tulving, E. (1983). Elements of Episodic Memory. 1st ed. Oxford: Oxford University Press.

Turrigiano, G. G. (2008). The Self-Tuning Neuron: Synaptic Scaling of Excitatory Synapses. Cell 135, pp. 422-435. DOI: 10.1016/j . cell.2008.10.008.

Turrigiano, G. G., Leslie, K. R., Desai, N. S., Rutherford, L. C., and Nelson, S. B. (1998). Activity-dependent scaling of quantal amplitude in neocortical neurons. Nature 391, pp. 892-896. DOI: 10.1038/36103.

Turrigiano, G. G. and Nelson, S. B. (2004). Homeostatic plasticity in the developing nervous system.. Nat. Rev. Neurosci. 5, pp. 97-107. DOI: 10.1038/nrn1327.

Van der Pol, B. (1920). A Theory of the Amplitude of Free and Forced Triode Vibrations. Radio Rev. 11, pp. 701-710.

Verdaasdonk, B. W., Koopman, H. F. J. M., and Helm, F. C. T. van der (2006). Energy efficient and robust rhythmic limb movement by central pattern generators. Neural Netw. 19, pp. 388-400. DOI: 10.1016/j . neunet.2005.09.003.

Verdaasdonk, B. W., Koopman, H. F. J. M., and Helm, F. C. T. van der (2009). Energy efficient walking with central pattern generators: from passive dynamic walking to biologically inspired control.. Biol. Cybern. 101, pp. 49-61. DOI: 10.1007/s00422009-0316-7.

Vogels, T. P., Sprekeler, H., Zenke, F., Clopath, C., and Gerstner, W. (2011). Inhibitory plasticity balances excitation and inhibition in sensory pathways and memory networks.. Science 334, pp. 1569-73. DOI: 10.1126/science. 1211095.

Walker, M. P., Brakefield, T., and Hobson, J. A. (2003). Dissociable stages of human memory consolidation and reconsolidation. Nature 425, pp. 616-620. DOI: 10 . 1038/nature01951.1..

Wall, M., Rechtsteiner, A., and Rocha, L. (2003). "Singular value decomposition and principal component analysis". A Practical Approach to Microarray Data Analysis. Ed. by D. P. Berrar, W. Dubitzky, and M. Granzow. Kluwer: Springer US, pp. 91109.

Wallén, P. and Williams, T. L. (1984). Fictive locomotion in the lamprey spinal cord in vitro compared with swimming in the intact and spinal animal. J. Physiol. (Lond.) 347, pp. 225-239. DOI: 10.1113/jphysiol.1984.sp015063.

Wang, T., Hu, Y., and Liang, J. (2013). Learning to swim: a dynamical systems approach to mimicking fish swimming with CPG. Robotica 31, pp. 361-369. DOI: 10.1017/ S0263574712000343.

Watanabe, K. and Funahashi, S. (2007). Prefrontal delay-period activity reflects the decision process of a saccade direction during a free-choice ODR task. Cereb. Cortex 17, pp. i88-i100. DOI: 10.1093/cercor/bhm102.

West, M. J. and Gundersen, H. J. G. (1990). Unbiased stereological estimation of the number of neurons in the human hippocampus. J. Comp. Neurol. 296, pp. 1-22. DOI: $10.1002 / \mathrm{cne} .902960102$.

Wilson, D. M. (1961). The Central Nervous Control of Flight in a Locust. J. Exp. Biol. 38, pp. 471-490.

Wiltgen, B. J., Brown, R. A. M., Talton, L. E., and Silva, A. J. (2004). New circuits for old memories: The role of the neocortex in consolidation. Neuron 44, pp. 101-108. DOI: $10.1016 / j$. neuron. 2004.09.015. 
Wimmer, K., Nykamp, D. Q., Constantinidis, C., and Compte, A. (2014). Bump attractor dynamics in prefrontal cortex explains behavioral precision in spatial working memory.. Nat. Neurosci. 17, pp. 431-439. DOI: 10.1038/nn. 3645.

Winfree, A. T. (1967). Biological rhythms and the behavior of populations of coupled oscillators.. J. Theor. Biol. 16, pp. 15-42. DOI: 10.1016/0022-5193 (67) 90051-3.

Wolff, M. J., Jochim, J., Akyürek, E. G., and Stokes, M. G. (2017). Dynamic hidden states underlying working-memory-guided behavior. Nat. Neurosci. 20, pp. 864871. DOI: $10.1038 / \mathrm{nn} .4546$.

Wolpert, D. M., Ghahramani, Z., and Flanagan, J. R. (2001). Perspectives and problems in motor learning. Trends Cogn. Sci. 5, pp. 487-494. DOI: 10.1016/S1364-6613(00) 01773-3.

Wood, R., Baxter, P., and Belpaeme, T. (2011). A review of long-term memory in natural and synthetic systems. Adapt. Behav. 20, pp. 81-103. DOI: 10.1177 / 1059712311421219.

Zenke, F., Agnes, E. J., and Gerstner, W. (2015). Diverse synaptic plasticity mechanisms orchestrated to form and retrieve memories in spiking neural networks. Nat. Commun. 6, p. 6922. DOI: $10.1038 /$ ncomms7922.

Zhang, W. and Linden, D. J. (2003). The other side of the engram: experience-driven changes in neuronal intrinsic excitability. Nat. Rev. Neurosci. 4, pp. 885-900. DOI: 10.1038/nrn1248.

Zhang, X., Yi, H., Bai, W., and Tian, X. (2015). Dynamic trajectory of multiple singleunit activity during working memory task in rats. Front. Comput. Neurosci. 9, p. 117. DOI: $10.3389 /$ fncom. 2015.00117.

Zhou, X., Zhu, D., Qi, X.-L., Lees, C. J., Bennett, A. J., Salinas, E., Stanford, T. R., and Constantinidis, C. (2013). Working memory performance and neural activity in prefrontal cortex of peripubertal monkeys.. J. Neurophysiol. 110, pp. 2648-60. DOI: 10.1152/jn.00370.2013.

Zucker, R. S. and Regehr, W. G. (2002). Short-term synaptic plasticity.. Annu. Rev. Physiol. 64, pp. 355-405. DOI: 10.1146/annurev.physiol.64.092501.114547. 


\section{Acknowledgements}

First of all, I would like thank my supervisor Dr. Christian Tetzlaff and Prof. Florentin Wörgötter for guiding and supporting my scientific work.

Furthermore, I would like to thank all my former and current colleagues in the Wörgötter-Lab for being such a lovely crowd and creating a pleasant and productive atmosphere in the group. I owe special thanks to my friends in the computational neuroscience subgroup (aka the "Tetzlab"), and here in particular to Juliane Herpich, Michael Fauth, Jannik Luboeinski and Johannes Auth not only for valuable scientific discussions and input but also for a wonderful time in the office, at conferences or in the pub.

My special thanks go to our secretary Ursula Hahn Wörgötter and to Antje Erdmann and Frauke Bergmann from the IMPRS PBCS office for ongoing indispensable support with all kinds of formalities and simply making things possible.

I would like to thank all my friends and my family for supporting me in all life situations. Without the continuous unconditional support and encouragement from my parents Karin and Klaus Nachstedt, this work and everything that preceded it would have been unthinkable. My biggest thanks, however, go to my love and wife Franziska for her emotional support during working on this thesis and simply for being the woman she is. I cannot imagine a more loveable, faithful and inspiring person than you to spend my life with.

Thank You All! 



\section{Timo Nachstedt}

\section{Date of Birth}

19.12.1987 in Clausthal-Zellerfeld, Germany

\section{E-Mail}

mail@nachstedt.com

Website

www.nachstedt.com

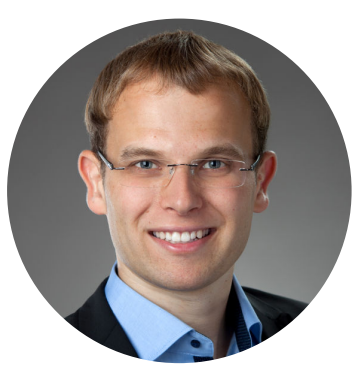

\section{Academical Education \& School}

Jan 2014 - Nov 2017

Göttingen, Germany

Oct 2010 - Mar 2013

Göttingen, Germany

Oct 2007 - Jul 2010

Göttingen, Germany

Jul 2000 - Jun 2007

Gehrden, Germany

\section{Doctorate}

International Max Planck Research School for Physics of Biological and Complex Systems, University of Göttingen

Focus: Neuronal mechanisms underlying the interaction of working and long-term memory

\section{Sc. Physics}

University of Göttingen

Focus: computational neuroscience and machine learning Master thesis: "Adaptive neuronal oscillator with synaptic plasticity for robotic locomotion control"

\section{B. Sc. Physics}

University of Göttingen

Focus: particle physics

Bachelor thesis: "Reconstruction of $\mathrm{Z}$ and Higgs bosons in tau final states with the ATLAS detector"

\section{Abitur}

Matthias-Claudius-Gymnasium

Advanced level subjects: physics, mathematics

\section{Practical Experiences}

Oct 2013 - Dez 2017

Göttingen, Germany

\section{Research assistant}

Third Institute of Physics

Organization of the student journal club and coordination of the research group seminar

Supervision of bachelor and master students 
Jan 2013 - Jun $2014 \quad$ Consultant

San Diego, CA, USA Brain Corp

Implementation of classical and neuronal algorithms for robotic locomotion control

Conception and implementation of a framework for virtual robot experiments

Remote activity with regular trips to the company headquarters, since October 2013 in part time

Jul 2011 - Dec 2011

Student assistant

Göttingen, Germany

Max Planck Institute for Dynamics and Self-Organization Implementation of a data management tool based on PHP and MongoDB

Jul 2009 - Apr 2011

Student assistant

Göttingen, Germany

Faculty of Physics

Supervision of students and training groups

Courses: physics I, basic physical practical course, basics of experimentation

Aug 2009 - Sep 2009 Intern

Gdynia, Poland

TeleMobile Electronics

Market analysis of the mobile network operators in Germany, Austria and Switzerland

Development and characterization of an LC bandpass filter with variable resonant frequency

\section{Stipends and Awards}

Jan 2014 - Dec 2016

Göttingen, Germany

Dec 2013

Göttingen, Germany

Dec 2010 \& Nov 2011

Göttingen, Germany

Dec 2010 \& Nov 2011

Hannover, Germany
Doctoral scholarship

International Max Planck Research School for Physics of Biological and Complex Systems

Dr. Berliner-Dr. Ungewitter-Award

Faculty of Physics, University of Göttingen

\section{Stipend of the Federal Country of Lower Saxony}

University of Göttingen

\section{Winner of the Apollo 13 student competition}

University of Hannover

\section{Journal Publications}

Nachstedt, T. and Tetzlaff, C.: Working memory requires a combination of transient and attractor-dominated dynamics to process unreliably timed inputs. Scientific Reports 7, 2017.

Nachstedt, T., Tetzlaff, C. and Manoonpong, P.: Fast dynamical coupling enhances frequency adaptation of oscillators for robotic locomotion control. Frontiers in 
Neurorobotics 11, 2017.

Chatterjee, S., Nachstedt, T., Tamosiunaite, M., Wörgötter, F., Enomoto, Y., Ariizumi, R., Matsuno, F. and Manoonpong, P.: Learning and Chaining of Motor Primitives for Goal-Directed Locomotion of a Snake-Like Robot with Screw-Drive Units. International Journal of Advanced Robotic Systems 12, 2015.

\section{Peer-Reviewed Conference Publications}

Ambe, Y., Aoi, S., Nachstedt, T., Manoonpong, P., Wörgötter, F., Matsuno, F.:

Embodied Sensorimotor Interaction for Hexapod Locomotion. Proceedings of the First International Symposium on Swarm Behavior and Bio-inspired Robotics, 2016.

Chatterjee, S., Nachstedt, T., Wörgötter, F., Tamosiunaite, M., Manoonpong, P., Enomoto, Y., Ariizumi, R. and Matsuno, F.: Reinforcement Learning Approach to generate Goal-directed Locomotion of a Snake-Like Robot with Screw-Drive Units. Proceedings of the 23rd International Conference on Robotics in Alpe-Adria-Danube Region (RAAD), 2014.

Ambe, Y., Nachstedt, T., Manoonpong, P., Wörgötter, F., Aoi, S. and Matsuno, F.: Stability analysis of a hexapod robot driven by distributed nonlinear oscillators with a phase modulation mechanism. Proceedings of the IEEE/RSJ International Conference on Intelligent Robots and Systems (IROS), 2013.

Nachstedt, T., Wörgötter, F., Manoonpong, P., Ariizumi, R., Ambe, Y. and Matsuno, F.: Adaptive neural oscillators with synaptic plasticity for locomotion control of a snake-like robot with screw-drive mechanism. Proceedings of the IEEE International Conference on Robotics and Automation (ICRA), 2013.

Nachstedt, T., Wörgötter, F. and Manoonpong, P.: Adaptive neural oscillator with synaptic plasticity enabling fast resonance tuning. Proceedings of the International Conference on Artificial Neural Networks (ICANN), 2012. 